\title{
Correlation Force Spectroscopy for Single Molecule Measurements
}

\author{
Milad Radiom \\ Dissertation submitted to the faculty of Virginia Polytechnic Institute \\ and State University in fulfillment of the requirements for the degree of \\ Doctor of Philosophy \\ In \\ Chemical Engineering
}

William A. Ducker, Chair

John Y. Walz

Mark Paul

Richey M. Davis

May 7, 2014

Blacksburg, VA

Keywords: Correlation Force Spectroscopy, Single Molecule Dynamics, Microrheology, Atomic Force Microscopy

(C) 2014: Milad Radiom All rights reserved 


\title{
Correlation Force Spectroscopy for Single Molecule Measurements
}

\section{Milad Radiom}

\begin{abstract}
This thesis addresses development of a new force spectroscopy tool, correlation force spectroscopy (CFS), for the measurement of the mechanical properties of very small volumes of material (molecular to $\mu \mathrm{m}^{3}$ ) at $\mathrm{kHz}-\mathrm{MHz}$ time-scales. CFS is based on atomic force microscopy (AFM) and the principles of CFS resemble those of dual-trap optical tweezers. CFS consists of two closely-spaced micro-cantilevers that undergo thermal fluctuations. Measurement of the correlation in thermal fluctuations of the two cantilevers can be used to determine the mechanical properties of the soft matter, e.g. a polymeric molecule, that connects the gap between the two cantilevers. Modeling of the correlations yields the effective stiffness and damping of the molecule. The resolution in stiffness is limited by the stiffness of the cantilever and the frequency by the natural frequency of the cantilevers, but, importantly, the damping resolution is not limited by the damping of the cantilever, which has enabled high-resolution measurements of the internal friction of a polymer. The concept of CFS was originally presented by Roukes' group in Caltech [Arlett et al., Lecture Notes in Physics, 2007]; I developed the first practical versions of CFS for experimentation, and have used it in two applications (1) microrheology of Newtonian fluids and (2) single molecule force spectroscopy. To understand the correlation in thermal fluctuations of two cantilevers I initially validated the theoretical approach for analyzing correlation in terms of deterministic model using the fluctuation-dissipation theorem [Paul and Cross, PRL, 2004]. I have shown that the main advantages of such correlation measurements are a large improvement in the ability to resolve stiffness and damping. Use of CFS as a rheometer was validated by comparison between experimental data and finite element modeling of the deterministic vibrations of the cantilevers using the known viscosity and density of fluids. Work in this thesis shows that the data can also be accurately fitted using a simple harmonic oscillator model, which can be used for rapid rheometric measurements, after calibration. The mechanical properties of biomolecules such as dextran and single stranded DNA (ssDNA) are also described.
\end{abstract}


CFS measurements of single molecule properties of ssDNA reveal the internal friction of the molecule in solution. 


\section{Acknowledgements}

My father, Mohammad Hossein, mother, Soheila, and brother, Soheil, receive my deepest gratitude for their patience, dedication, and years of emotional support. What I received from them through years: endless love, support, and encouragement, were in the end what made this dissertation and all that I achieved possible. My perseverance, motivation, and ambition in this $\mathrm{PhD}$ thesis were due to a supporting family that accompanied me every single day in the past few years, although miles and miles away, but living with me and in my heart every single minute.

It is not easy to put into words, but simply, the years that I spent as a $\mathrm{PhD}$ student in the Department of Chemical Engineering at Virginia Tech have been the most inspiring and promising years of my life. I owe this invaluable experience to the unique atmosphere of Virginia Tech, but to a much greater extend, to my supervisor, Professor William Ducker. Working with this prominent scientist and researcher, and learning from him, were not only highlights of an academic life, but advent of a new lifestyle. This is due to his special character and charismatic behavior. His insights, ideas, perseverance and encouragements were great source of success in this project.

I would like to thank Professor Mark Paul from Department of Mechanical Engineering and Professor John Walz from Department of Chemical Engineering ${ }^{1}$ for their guidance, support and advice. I am very grateful for all their assistance in this project. Especially, to Professor Mark Paul for his close collaboration and his insights on the theoretical part of this project and development of analytical solutions. Especial thanks are due to Professor Mark Paul and Mr. Brian Robbins for the finite element simulation results presented in the microrheology chapter. Special thanks are also due to Dr. Chris Honig for his critical contribution to the development of the laterally offset correlation force spectroscopy. I am also very grateful to Professor Richey Davis from Department of Chemical Engineering for his continual support and advice to this thesis.

\footnotetext{
${ }^{1}$ Currently Dean of Engineering at University of Kentucky
} 
I would like to thank Mr. Riley Chan and Mr. Michael Vaught for their contributions in building the apparatus.

I have gained a lot of confidence and experience in my research by closely working with Mr. Dongjin Seo, Mr. Dean Mastropietro and Dr. Dmitri Iarikov, Dr. Adam Bowles, Ms. Ching-Wen Chiu, Ms. Ratthaporn Chatchaidech, and Dr. Nathan Nicholas. 
I dedicate this thesis to my father, Mohammad Hossein Radiom, mother, Soheila Askarzadeh, and brother, Soheil Radiom. They are the real meaning to all that I achieve.

I dedicate this thesis to my PhD advisor, Professor William Ducker, for what he taught me, and for that he trusted me. 


\section{Table of Contents}

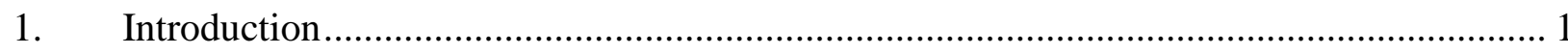

Overview of Single Molecule Force Spectroscopy Techniques.................................................... 3

Experiments Using Single molecule AFM ....................................................................... 4

Limitations of AFM-based single molecule force spectroscopy …….......................................... 6

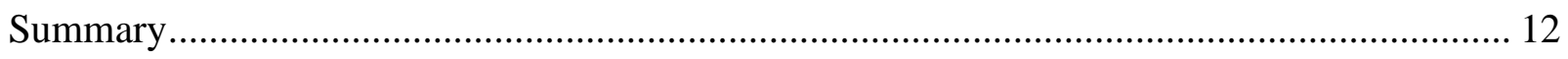

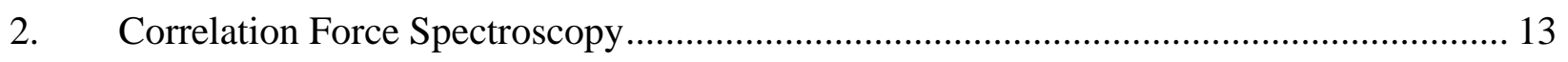

Correlation Force Spectroscopy: Rationale …………….................................................. 13

Correlation Force Spectroscopy: Development ................................................................... 15

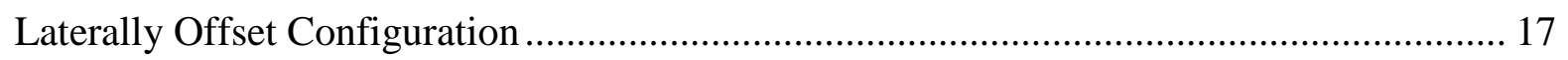

Vertically Offset Configuration ................................................................................... 18

Analysis of Correlations between Two Cantilevers .................................................................. 22

Validation of Fluctuation-Dissipation Theorem for One Cantilever ...................................... 24

Analysis of Thermal Fluctuations to obtain Correlations......................................................... 25

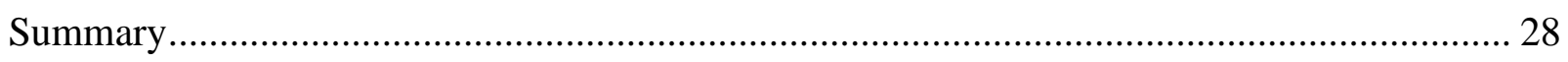

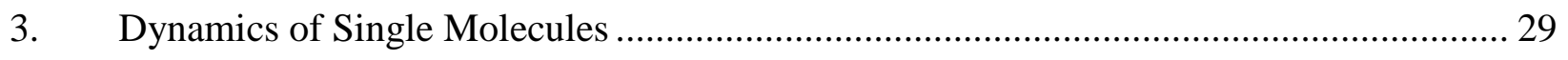

Elastic Properties of Single Molecules: Worm-Like Chain and Freely-Jointed Chain Models 29 Hydrodynamics of Single Molecules: Dumbbell Model and Rouse Model............................... 33

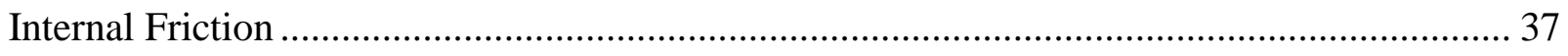

Rouse with Internal Friction ............................................................................................ 41

Model of Linear Viscoelasticity of a Semiflexible Chain ......................................................... 42

Summary

4. Microrheology with Correlation Force Spectroscopy …………………………............... 45 


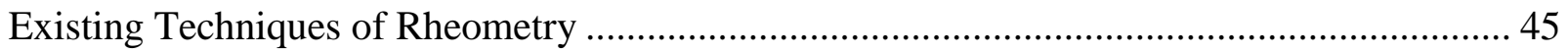

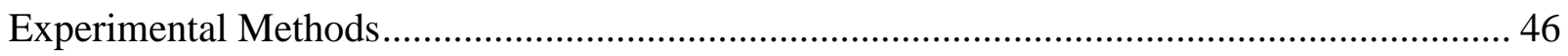

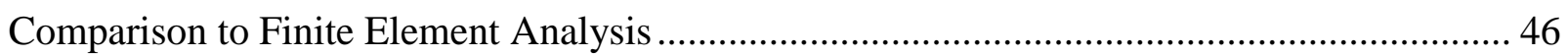

Comparison to Simple Harmonic Oscillator Model ………………......................................... 48

Summary

5. Development of Colloidal Probe Correlation Force Spectroscopy: Case Study ................ 54

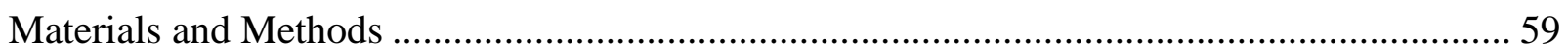

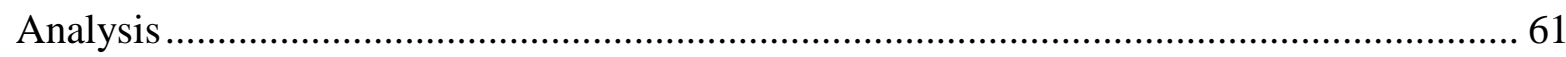

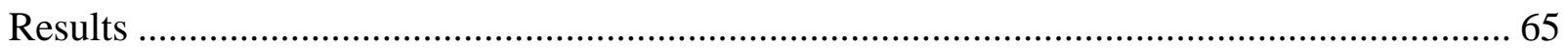

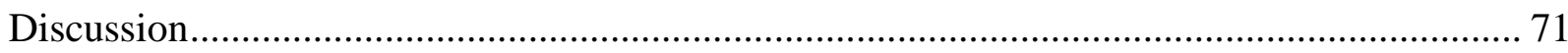

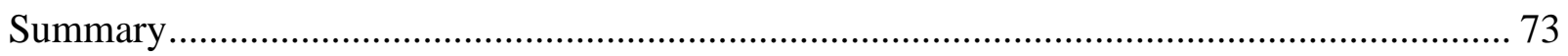

6. Correlation Force Spectroscopy for Single Molecule Measurements ................................ 75

Effect of the Distance between Cantilever Tips ................................................................. 75

Harmonic Oscillator Modeling of Vertically Offset Correlation Force Spectroscopy.............. 77

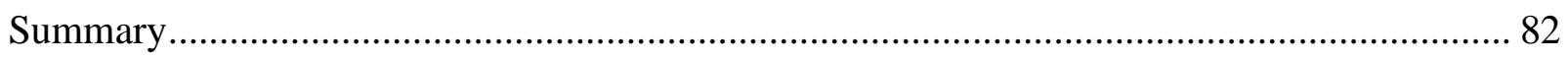

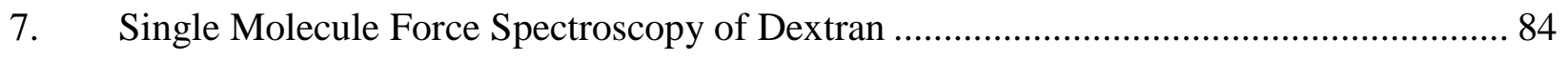

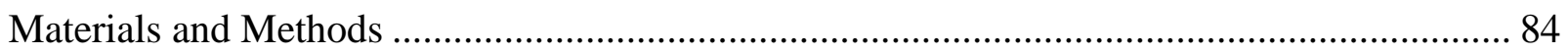

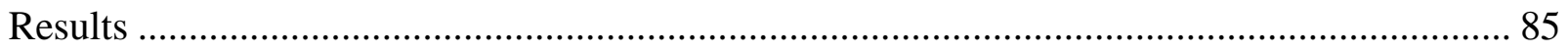

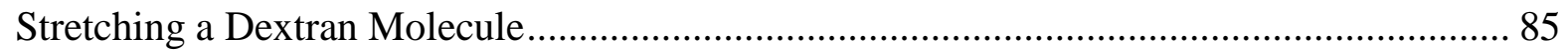

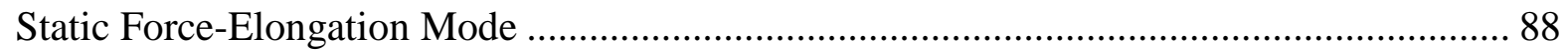

Dynamic Correlated Fluctuations Mode ………………....................................................... 92

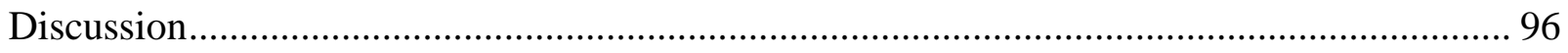

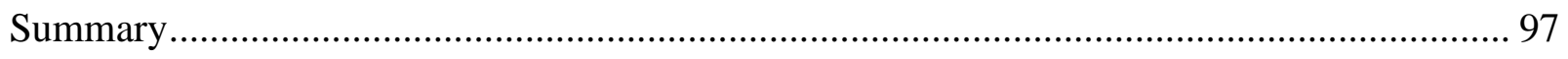

8. Single Molecule Force Spectroscopy of Single-Stranded DNA ………………………....... 98

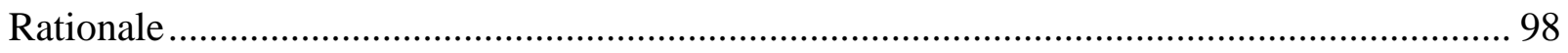




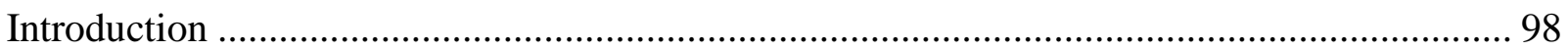

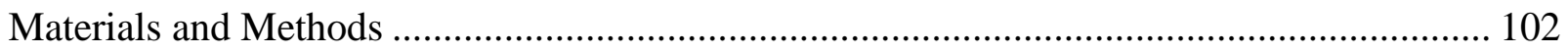

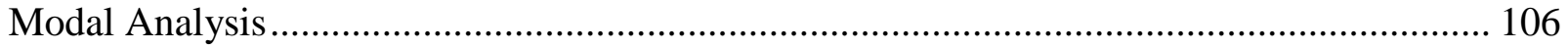

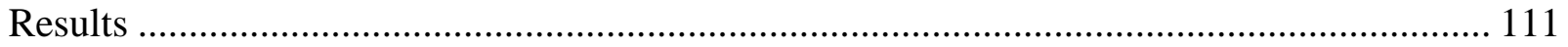

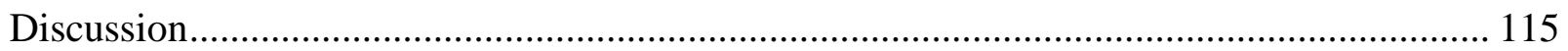

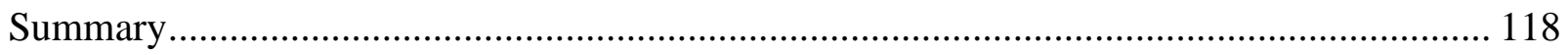

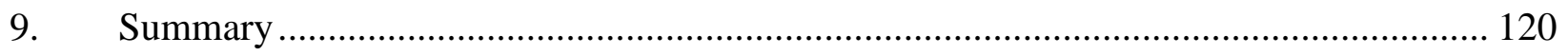

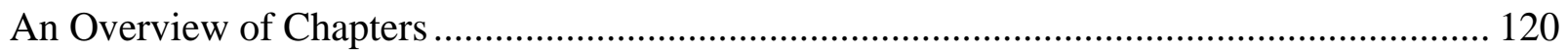

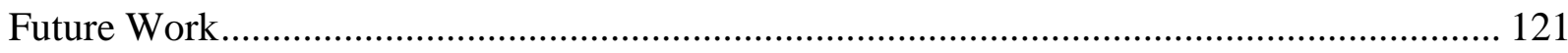

Appendix I: Simple Harmonic Oscillator Model for Laterally Offset Correlation Force

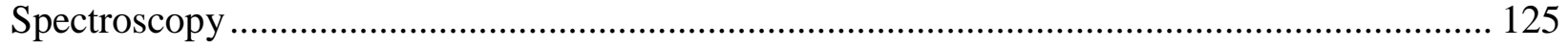

Appendix II: Simple Harmonic Oscillator Model for Vertically Offset Correlation Force Spectroscopy with Tethered Molecule .......................................................................... 127

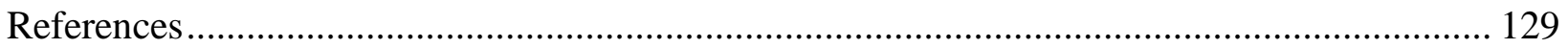




\section{List of Figures}

Figure 1-1: Schematic of AFM with viscous friction $\gamma_{\mathrm{a}}$ and CFS with viscous friction $\gamma_{\mathrm{c}}$. $\gamma_{\mathrm{c}} \ll \gamma_{\mathrm{a}}$ due to much smaller area of inter-cantilever hydrodynamic interaction. This results in much better force resolution in CFS than AFM. 10

Figure 2-1: Comparison between the normalized autocorrelation noise spectrum $\left(G_{11}^{\prime}\right)$ and the normalized cross-correlation noise spectrum $\left(G_{12}^{\prime}\right)$ in water at $23^{\circ} \mathrm{C}$. Cantilevers used are ORC8-B: length $=200 \mu \mathrm{m}$, width $=40 \mu \mathrm{m}, \mathrm{k}=0.1 \mathrm{Nm}^{-1}$. The separation between the cantilevers is $d=6.5 \mu \mathrm{m}(s=318 \mathrm{~nm})$ in the vertically offset CFS as shown in Figure 2-4-b.

Figure 2-2: Experimental measurements of $\gamma_{\mathrm{a}}$, the viscous friction on a single cantilever and $\gamma_{\mathrm{c}}$, the viscous friction between two closely spaced cantilevers, as a function of distance. $\gamma_{c}$ is much smaller... 15

Figure 2-3: (a) Schematic of the cantilevers and detection system in the antiparallel laterally offset CFS. (b) Light microscope images of closely-spaced AFM cantilevers seen from above. The cantilevers are $100 \mu \mathrm{m}$ long and $40 \mu \mathrm{m}$ wide. The lateral separation between the cantilevers is $8 \mu \mathrm{m}$.

Figure 2-4: (a) Atomic force microscopy contact mode image in water of the bottom cantilever tip obtained by scanning the bottom cantilever and measuring the height signal. This imaging is used to align the tips. (b) Schematic of antiparallel vertically offset cantilevers. (c) Schematic of the cantilevers and detection system in the antiparallel vertically offset CFS. 18

Figure 2-5: Top view optical microscope images of the cantilever pair. (Top) The two cantilevers are brought in proximity of each other. (Bottom) The two cantilevers are aligned coarsely using micrometer translation stage and then with nanometer precision using the piezoelectric devices of the AFM. 
Figure 2-6: Schematic representation of equation (2.5). $x_{1}$ and $x_{2}$ are the thermal fluctuations of the top and bottom cantilever respectively and $X_{2}$ is the deterministic deflection of the bottom cantilever after removal of force $F_{1}$ from the top cantilever. 23

Figure 2-7: Comparison of the left and right sides of equation (2.4). The autocorrelation (normalized by $k_{\mathrm{B}} T / k_{\mathrm{c}}$ ) and the deflection (normalized by its value at time zero) after release of a step force are plotted on the same axis. Both data sets were collected at $1 \mathrm{MHz}$ with ORC8-B type cantilever with spring constant $0.1 \mathrm{~N} / \mathrm{m}$ and resonant frequency $5 \mathrm{kHz}$ in water. 24

Figure 3-1: Bond angle $\theta_{i}$ between bond vectors $\overrightarrow{r_{i}}=\overline{C_{i-1} C_{i}}$ and $\overline{C_{i} C_{i+1}}$ and torsion angle $\varphi_{i}$ of bond vector $\overline{C_{i} C_{i+1}}$ where $\vec{r}_{i}$ is the axis of rotation. There is energy cost associated with variation of torsion angle $\varphi_{i}$ due to variation in distance between atoms $C_{i-2}$ and $C_{i}$. $\left|\overrightarrow{r_{i}}\right|=\left|\vec{r}_{j}\right|=l$ is the bond length. 30

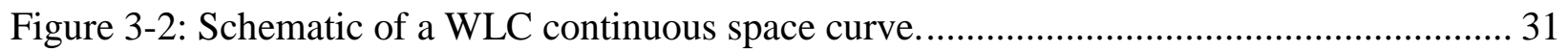
Figure 3-3: (a) Schematic of a dumbbell. (b) Schematic of a spring with stiffness $k$ and dashpot with friction coefficient $\zeta_{\mathrm{s}}$. This is the model of a highly overdamped simple harmonic oscillator where inetial foces due to mass are ignored. 34

Figure 3-4: Rouse model consists of $N$ beads of bead-solvent friction $\zeta_{\mathrm{s}}$ that are connected by linear springs of stiffness $k^{\prime}$. 35

Figure 3-5: (a) Schematic of the smooth energy landscape $U$ which varies along the reaction coordinate $x . A$ is the minimum energy and $s t$ is the transition state. $\Delta F_{0}^{\neq}$is the activation energy to cross the barrier. (b) Schematic of the rough energy landscape $U$ with root mean squared roughness $\varepsilon$

Figure 3-6: Schematic of RIF model. The beads are connected with springs of stiffness $k^{\prime}$ and dashpots of friction $\zeta_{i}$ (internal friction). 41

Figure 4-1: Autocorrelation of equilibrium fluctuations in cantilever displacement in a series of Newtonian fluids. Experimental measurements at $23^{\circ} \mathrm{C}$ are shown as data symbols and theoretical predictions are shown by the solid lines. Commercial AFM cantilevers are used for the experiments (ORC8 B: length $=200 \mu \mathrm{m}$, width $=40 \mu \mathrm{m}, \mathrm{k}=0.1 \mathrm{Nm}^{-1}$ ). The 
simulations used a cantilever with a stepped end as shown in the inset. The prime on the left axis indicates normalized correlation function. 47

Figure 4-2: Cross-correlation of equilibrium fluctuations in cantilever displacement for a pair of AFM cantilevers in a series of Newtonian fluids. Experimental measurements are shown as data symbols and theoretical predictions are shown by the solid lines. Commercial AFM cantilevers are used (ORC8 B: length $=200 \mu \mathrm{m}$, width $=40 \mu \mathrm{m}, \mathrm{k}=0.1 \mathrm{Nm}^{-1}$ ). Experiments were performed at $23^{\circ} \mathrm{C}$. The cantilevers are separated by $8 \mu \mathrm{m}$. The prime on the left axis indicates normalized correlation function. 48

Figure 4-3: Schematic of a cantilever pair modeled as a mass-spring-damper system. $m$ is the effective (i.e. averaged over all frequencies) fluid loaded mass of the cantilever, $k_{c}$ is the spring constant, and $\gamma_{\mathrm{a}}$ is the coefficient of fluid damping on an individual cantilever. $\gamma_{\mathrm{c}}$ is the coefficient of fluid damping due to the fluid spanning the gap between the two cantilevers. The dashpot for $\gamma_{\mathrm{c}}$ works such that when the left mass moves up the right mass experiences a force in the downward direction, and vice versa. The plates shown in the schematic are massless.

Figure 4-4: Comparison of HO model with experimental data. (a) and (b) auto- and crosscorrelations in n-pentane. (c) and (d) auto- and cross-correlations in water. Each plot shows the experimental results, the harmonic oscillator (HO) fit and the finite element (FE) model (using the known liquid viscosity and density). The ringdown from the HO and FE models have been used to yield the correlations in fluctuations using equations (2.4) and (2.5). These results are for a pair of commercial AFM cantilevers (ORC8 B: length $=200 \mu \mathrm{m}$, width $=40 \mu \mathrm{m}, \mathrm{k}=0.1 \mathrm{Nm}^{-1}$ ) separated by $8 \mu \mathrm{m}$ at $23^{\circ} \mathrm{C}$. 52

Figure 5-1: Schematic of the experiment. $a$ is the radius of a sphere, $D$ is the separation between the two spheres, and the distance between the spheres is $r=D+2 a$. The dimensionless center-to-center distance is $\rho=r / a . x_{1}$ and $x_{2}$ are the thermally-stimulated deflections of the cantilever-sphere assemblies that are detected by individual detection systems. The zpiezo in the top assembly is part of the commercial AFM and is used to vary the separation between the two spheres. In collective (symmetric) motion, the spheres move in the same direction while in relative (asymmetric) motion, they move in opposite directions. 59 
Figure 5-2: Approach and retract force-separation curves obtained by driving the top cantileversphere assembly at $1000 \mathrm{~nm} \mathrm{~s}^{-1}$ toward, and then away, from the bottom assembly. The overlay of the approach and retract curves shows that the lubrication force is very small compared to time-independent forces such as the double-layer forces. The inset shows the measured deflection of the top and bottom assemblies are equal and opposite. 61

Figure 5-3: Power spectral density of the collective motion, $G_{s}$, and power spectral density of the relative motion, $G_{a s}$, at varying separations $D$. Only three separations are shown in the top graph, $D=\infty, 4 \mu \mathrm{m}$, and $10 \mathrm{~nm}$. Decreasing separations in the bottom graph are $D=\infty$ , $14 \mu \mathrm{m}, 11 \mu \mathrm{m}, 7 \mu \mathrm{m}, 4 \mu \mathrm{m}, 2 \mu \mathrm{m}, 400 \mathrm{~nm}, 150 \mathrm{~nm}, 100 \mathrm{~nm}, 30 \mathrm{~nm}$, and $10 \mathrm{~nm}$. 65

Figure 5-4: Values of $\zeta_{s}$ and $\zeta_{a s}$ obtained from fits to equation (5.15) and (5.16) using the data shown in Figure 5-3. Data is plotted as a function of the dimensionless separation $(\rho=r / a)$. Results are shown for two separate experiments. 67

Figure 5-5: Cross-correlation spectral density, $G_{12}$, at varying separations $D$. Decreasing separations are $D=\infty, 14 \mu \mathrm{m}, 11 \mu \mathrm{m}, 7 \mu \mathrm{m}, 4 \mu \mathrm{m}, 2 \mu \mathrm{m}, 400 \mathrm{~nm}, 150 \mathrm{~nm}, 100 \mathrm{~nm}, 30 \mathrm{~nm}$, and $10 \mathrm{~nm}$. Solid lines show the quality of fits for separations $D=\infty, 4 \mu \mathrm{m}, 2 \mu \mathrm{m}, 30 \mathrm{~nm}$, and $10 \mathrm{~nm}$. At any given frequency, the magnitude of $G_{12}$ changes monotonically with separation. 68

Figure 5-6: Friction of single sphere motion $\zeta_{11}^{*}$, and friction of mutual motions of two spheres, $\zeta_{12}^{*}$, as a function of dimensionless separation $\rho=r / a$. (Note, that I actually plot ${ }_{12}^{*}$.) Filled and hollow circles represent two different experimental data sets. Experimental data are compared with expressions (5.21) and (5.22) for small frequency number $\operatorname{Re}_{\omega}$ and large separations $\rho$ and expressions (5.23) and (5.24) for small frequency number $\operatorname{Re}_{\omega}$ and small separations ${ }^{6}$. Equation (5.4) is the high frequency number friction on a free sphere. Data are normalized by $6 \pi \eta a$. 70

Figure 5-7: Normalized cross-correlation function $\left(\pi r \eta \omega^{2} / k_{B} T\right) G_{12}$ compared for several separations with theoretical expression $e^{-z} / z^{2}[(1+z) \sin (z)-z \cos (z)]$ in equation (5.6) and plotted versus the dimensionless ratio $z=r / \delta$. For each experimental data set, $r$ and $v$ are fixed, so $r / \delta$ is a function of frequency only. 72 
Figure 5-8: The current experiment reduces the hydrodynamic coupling between cantilevers by keeping them well separated and antiparallel (left). A conventional drainage experiment could achieve a similar effect by attaching the bottom sphere to a step edge (right).......... 73

Figure 6-1: (a) Schematic of antiparallel vertically offset cantilevers. Tip height is $h$, thus $d=s+$ 2h. (b) Schematic figure showing a molecule being stretched between the tips. 75

Figure 6-2: (a) Autocorrelation and (b) cross-correlation of equilibrium fluctuations in cantilever deflection in water at $23^{\circ} \mathrm{C}$. A series of measurements is shown for various tip-tip separations, $s=318-7300 \mathrm{~nm}$, which corresponds to a cantilever-cantilever separation, $d=$ 6.5-13.5 $\mu \mathrm{m} . \mathrm{d}=6.5 \mu \mathrm{m}$ and $\mathrm{d}=13.5 \mu \mathrm{m}$ are labeled and the rest of separations are in sequential order. Commercial AFM cantilevers are used (ORC8 B: length $=200 \mu \mathrm{m}$, width $=40 \mu \mathrm{m}, \mathrm{k}=0.1 \mathrm{Nm}^{-1}$ ). The prime on the left axis indicates normalized correlation function. Note that the autocorrelation data for different separations almost overlay: there is a slight decrease in amplitude at smaller separations. The cross-correlation increases monotonically as the separation decreases. Fluctuations are collected at $50 \mathrm{kHz}$ for $50 \mathrm{sec}$. using the Asylum Research MFP3D controller. Discrete points have been represented as a line for clarity. 77

Figure 6-3: Schematic of a cantilever pair modeled as a mass-spring-damper system. $m$ is the effective fluid loaded mass of the cantilever, $k_{\mathrm{c}}$ is the spring constant, and $\gamma_{\mathrm{a}}$ is the coefficient of fluid damping on an individual cantilever. $\gamma_{\mathrm{c}}$ is the coefficient of fluid damping due to the fluid spanning the gap between the two cantilevers. The plates shown in the schematic are massless. 78

Figure 6-4: Auto- and cross-correlation of equilibrium fluctuations in cantilever displacement for a pair of AFM cantilevers in water at $23^{\circ} \mathrm{C}$. (a) is autocorrelation at large separation, (b), (d) and (f) are autocorrelations for a series of separations, (c), (e) and (g) are cross-correlations for a series of separations. Commercial AFM cantilevers are used (ORC8 B: length = 200 $\mu \mathrm{m}$, width $=40 \mu \mathrm{m}, \mathrm{k}=0.1 \mathrm{Nm}^{-1}$ ). Experiment is the data measured by CFS, and HO model were calculated using the fluctuation-dissipation theory to model fluctuations via cantilever ringdown and a coupled harmonic oscillator $(\mathrm{HO})$ model for the ringdown. The prime on the left axis indicates normalized correlation function. 82 
Figure 6-5: (a) Fitted damping coefficient $\gamma_{c}$ as a function of separation between the cantilevers.

(b) The force noise amplitude due to fluid coupling $\left(F_{12}\right)$ versus distance between the two cantilevers. 82

Figure 7-1: Schematic presentation of LVDT and deflection signals. 86

Figure 7-2: (a) Top cantilever, (b) bottom cantilever deflection signals. Bottom cantilever has a noisier background. The zero of deflection voltage is arbitrary. 87

Figure 7-3: (a) Top cantilever, (b) bottom cantilever deflection signals filtered at $500 \mathrm{~Hz}$. x-axis is LVDT signal also filtered at $500 \mathrm{~Hz}$ and converted to units (m) using LVDT sensitivity factor. 88

Figure 7-4: Deflection of top and bottom cantilever as a function of LVDT in dextran experiments. 90

Figure 7-5: Force-polymer extension curve of dextran molecule. The rupture of the final dextran bond to the tip is at about 40, 80, and $150 \mathrm{~nm}$ (from top to bottom). 91

Figure 7-6: Values of $G_{12, s}$ and $G_{12, a s}$ obtained from experiment (symbols) for (a) $578 \mathrm{~nm}$ tip-totip separations (without polymer) and (b) $152 \mathrm{~nm}$ tip-to-tip separation (straddling polymer). Fits to equations (7.9) to (7.12) (lines) are also shown, using $m, k, \gamma_{\mathrm{a}}, \gamma_{\mathrm{c}, \mathrm{fl}}, k_{\mathrm{p}}$ and $\zeta_{\mathrm{p}}$. A different fit is used for the experiment (a) and (b). 94

Figure 7-7: (a) Stiffness of dextran obtained from dynamic correlated fluctuations (CF) mode, static force-elongation mode, fluctuations $(F)$ mode of a single cantilever and compared with results of Kawakami, et al. [36] as a function of force. (b) Friction of dextran obtained from dynamic correlated fluctuations (CF) mode, and fluctuations (F) mode of a single cantilever and compared with results of Kawakami, et al. [36] as a function of force. 96

Figure 8-1: Schematic of CFS experiment. $\langle R\rangle$ is the average molecule elongation. $x_{1}$ and $x_{2}$ are the thermal fluctuations in displacement of the cantilevers that are detected by individual detection systems. A z-piezo drive mechanism is used to vary $c$ and thus to alter the elongation of the molecule. A PID feedback loop is used to maintain a constant force $F$ on the molecule during the force clamp period. The schematic shows a molecule of ssDNA on each tip bound to each other in a short base-pairing section. 102

Figure 8-2: Force-elongation of ssDNA obtained using AFM, and low pass-filtered $(\mathrm{f}=500 \mathrm{~Hz})$ to show quasi-static force. Both approach (grey) and retract (black) curves are shown using 
dotted lines. The solid line shows a linear fit to force-elongation data which results in static stiffness. 104

Figure 8-3: Force-LVDT curve of ssDNA pull and clamp in the dynamic force-clamp mode of CFS. In this experiment, $c$ is increased with time (as measured by the LVDT) while the force is measured. When the force reaches a preset threshold (horizontal arrow), a feedback loop is automatically activated to maintain a constant force. This force is shown by the horizontal arrow in both the main figure and the inset. The inset is the force-time curve of ssDNA during the pull and the clamp. The left vertical arrow in the inset shows the start of the force-LVDT curve in the parent figure $(\mathrm{LVDT}=1.06 \mu \mathrm{m})$. The right vertical arrow shows the time at which the dsDNA splits apart and the molecule no longer spans between the two tips. The LVDT and time values on horizontal axes are arbitrary. The start of the clamp period is labeled by the horizontal arrow in the inset. 105

Figure 8-4: Schematic of the analysis. Each cantilever is modeled as a simple harmonic oscillator with mass $m$, stiffness $k_{\mathrm{c}}$, and damping $\zeta_{11} \cdot \zeta_{12}$ is the hydrodynamic fluid coupling between the cantilevers. $k_{\mathrm{ssDNA}}$ and $\zeta_{\mathrm{ssDNA}}$ are the stiffness and friction coefficient of the DNA straddling between the two cantilevers. The directions of the symmetric and asymmetric modes are shown 106

Figure 8-5: Symmetric component of the correlation power spectral density in the clamped region (full circle) and in post-melt region (open circle). Results of only one experiment is shown 108

Figure 8-6: Asymmetric-correlation power spectral density in the clamped region (circle) and in post-melt region (square). Different colors show different experiments. Solid lines show the quality of fits of equation (8.11) to experiment. Three lines are drawn in color to match the same color experimental symbols. Equation (8.11) is fitted to experimental $G_{\text {as }}$ from 600 $\mathrm{Hz}$ to $4 \mathrm{kHz}$. 110

Figure 8-7: Stiffness of ssDNA measured in dynamic force-clamp mode of CFS (open circle) and static force-elongation mode of AFM (filled square). Solid line and dashed line are WLC model equation (8.2) and (8.4) respectively with $R_{\max }=42 \mathrm{~nm}$ and $l_{\mathrm{p}}=2.5 \mathrm{~nm}$. 113

Figure 8-8: Internal friction coefficient of ssDNA measured in dynamic force-clamp mode of CFS. Solid line is fit of FWLC model equation (8.5) with $R_{\max }=42 \mathrm{~nm}$ and $l_{\mathrm{p}}=2.5 \mathrm{~nm}$ 
and fitted value $\zeta_{\mathrm{B}}=11 \mu \mathrm{g} \mathrm{kHzm^{3 }}$. The horizontal line shows the error in force. The error in damping is unknown as we cannot do repeat measurements of the same chain............. 115

Figure 8-9: Relaxation time of ssDNA calculated from the ratio of $\zeta_{\mathrm{ssDNA}}$ to $k_{\mathrm{sDNA}} \ldots \ldots \ldots \ldots \ldots . .116$ 


\section{List of Tables}

Table 1-1: Comparison of techniques used for single molecule force spectroscopy $[1,8,9]$...... 4 Table 4-1: Fit parameters for HO model of the two-cantilever system. $\gamma_{\mathrm{a}, \text { theory }}$ is calculated from equation (29) in [43]. $m_{\mathrm{e}}$ is the fluid loaded mass of the cantilever in air, $m_{\mathrm{e}}=k_{\mathrm{c}} / \omega_{0}^{2}$, where $\omega_{0}$ is the resonant frequency in air. The viscosity, $\eta$, was measured using a controlled shear

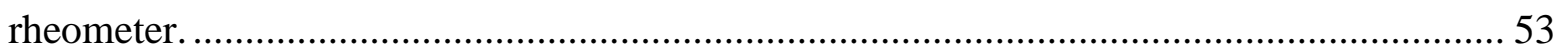

Table 8-1: Roughness of energy landscape in unfolding-folding of molecules. ..................... 118 


\section{Nomenclature}

\section{Roman Symbols}

$\begin{array}{ll}a & \text { Radius } \\ b & \text { Kuhn length } \\ D & \text { Rayleigh dissipation function } \\ F & \text { Force } \\ f_{n} & \text { Langevin force } \\ f & \text { Characteristic force } \\ \mathrm{f} & \text { Frequency } \\ G & \text { Power spectral density } \\ k & \text { Stiffness } \\ k_{\mathrm{B}} & \text { Boltzmann's constant } \\ R_{\max } & \text { Contour length } \\ l_{\mathrm{p}} & \text { Persistence length } \\ N & \text { Number of Kuhn segments } \\ \langle R\rangle & \text { End-to-end distance of polymer } \\ p & \text { Pressure } \\ Q & \text { Quality factor } \\ T & \text { Absolute temperature } \\ t & \text { Time }\end{array}$

\section{Greek Symbols}

$\Gamma$

$\gamma_{\mathrm{a}}$

$\gamma_{\mathrm{c}}$

$\gamma_{\mathrm{c}, \mathrm{fl}}$

$\gamma_{\mathrm{c}, \mathrm{p}}$

$\delta$
Hydrodynamic function

Friction coefficient of autocorrelation

Friction coefficient of cross-correlation

Friction coefficient due to fluid coupling two cantilevers

Friction coefficient due to polymer coupling two cantilevers

Dirac delta function 


\begin{tabular}{ll}
$\delta_{\mathrm{s}}$ & Wake of unsteady viscous boundary layer \\
$\zeta$ & Friction coefficient \\
$\zeta_{\mathrm{B}}$ & Bending friction coefficient \\
$\zeta_{i}$ & Polymer internal friction \\
$\zeta_{\mathrm{s}}$ & Polymer-solvent friction \\
$\eta$ & Viscosity \\
$\theta$ & Bond angle \\
$\kappa_{\mathrm{B}}$ & Bending modulus \\
$v$ & Kinematic viscosity \\
$\rho$ & Dimensionless interparticle separation \\
$\rho_{\mathrm{f}}$ & Fluid density \\
$\tau$ & Relaxation time constant \\
$\varphi$ & Torsion angle \\
$\chi$ & Compliance \\
$\omega$ & Angular frequency \\
Units & \\
\hline
\end{tabular}

$\begin{array}{ll}k_{\mathrm{B}} T & \text { Boltzmann's energy factor }(\cong 4 \mathrm{pN} \mathrm{nm}) \\ \mathrm{nm} & 10^{-9} \mathrm{~m} \\ \mathrm{pN} & 10^{-12} \mathrm{~N} \\ \mathrm{pN} \mathrm{nm} & 10^{-3} \mathrm{~N} \mathrm{~m}^{-1} \\ \mu \mathrm{g} & 10^{-9} \mathrm{~kg}^{-6} \mathrm{~kg} \mathrm{~s}^{-1} \\ \mu \mathrm{g} \mathrm{kHz} & 10^{-6} \mathrm{p}^{-1}\end{array}$

\section{Acronyms}

$\begin{array}{ll}\text { AFM } & \text { Atomic Force Microscopy } \\ \text { BW } & \text { Bandwidth } \\ \text { CFS } & \text { Correlation Force Spectroscopy } \\ \text { DNA } & \text { Deoxyribose Nucleic Acid } \\ \text { ssDNA } & \text { Single Stranded Deoxyribose Nucleic Acid } \\ \text { FDT } & \text { Fluctuation Dissipation Theorem } \\ \text { FE } & \text { Finite element }\end{array}$


FFJC

FJC

FT

FWLC

InvOLS

MT

OT

PE

PSD

RIF

rms

RNA

SHO

SMFS

WLC
Frictional Freely-Jointed Chain

Freely-Jointed Chain

Fourier Transform

Frictional Worm-Like Chain

Inverse optical lever sensitivity

Magnetic tweezers

Optical tweezers

Polymer extension

Power Spectral Density

Rouse with Internal Friction

Root Mean Square

Ribose Nucleic Acid

Simple Harmonic Oscillator

Single molecule force spectroscopy

Worm-Like Chain 


\section{Introduction}

"Almost all aspects of life are engineered at the molecular level, and without understanding molecules we can only have a very sketchy understanding of life itself."

Francis Crick (1988)

"Function [of biomolecules] arises from conformation"

Stryer (1981)

Force is an important parameter that defines function and structure for many polymeric molecules [1]. For some biopolymers, such as muscle proteins, the application of force is the principal function. In other cases, the forces are important during the adoption and preservation of the native structure (e.g. protein folding) or during their action (e.g. enzyme catalysis). In all cases, the application of a force can alter the structure of the molecule and therefore may affect its function and activity [2]. Under an applied force, molecules express mechanical properties, e.g. stiffness (resistance to extension) and friction (resistance to velocity). The mechanical properties of molecules can be studied on collections of molecules or on individual molecules. In some cases it is preferable to study large collections, such as whole cells or muscles, but very often there is no collective motion and so the corresponding mechanical properties of the collection have no significance. For example, many enzymes are single molecules: catalysis applies forces at the individual molecule level and there is no meaning to the force applied by many enzymes (they are unlikely even to act in the same direction). Many transmembrane proteins also act individually or in small collections. Therefore, it is essential to study these molecules under the application of force and at single molecule level.

The study of single molecules has specific application in medicine: diseases such as cancer metastasis, or biological processes such as inflammation, involve highly regulated molecular 
events that can only be well understood if evidence of specific molecular participation in the complex biological phenomenon is obtained; this however requires studying behavior of individual molecules [3]. There is also an entire class of diseases, the protein misfolding diseases, Huntington's, Parkinson's, Alzheimer's and others, that are thought to be due to "nonfunctional" conformations of proteins [4-7]. The adoption of the functional form of proteins depends on the forces applied during folding.

For this reason, a new generation of research tool, single molecule force spectroscopy (SMFS), has been developed to explore the properties of single polymeric molecules. Most SMFS experiments are mechanical, that is, the molecule is under an applied force while its properties are being probed. There are specific merits in studying molecules using mechanical SMFS. One example is the well-defined reaction coordinate in the direction of the applied force. In contrast, biological molecules undergo thermal and chemical denaturation with a complex reaction coordinate: the disadvantage of SMFS is that it does not explore all this complexity, but the advantage is that the reaction coordinate is known and in a single dimension [8].

A variety of single molecule techniques have been developed. The focus of this thesis is force measurements, so in the next section I will briefly review the techniques of mechanical single molecule force measurement [1] to set the background for the principal topic of this thesis: the development of a new single molecule force technique, correlation force spectroscopy. A number of techniques differing in force and frequency bandwidth are available: magnetic beads, optical tweezers, glass microneedles, biomembrane force probe and atomic force microscopy (AFM). AFM-based single molecule force spectroscopy is most popular due to its ease of operation, range of accessible force (pico- to nano-Newton) and bandwidth $(1 \mathrm{~Hz}-100 \mathrm{kHz})$. I will then introduce Correlation Force Spectroscopy (CFS), the method that I developed to enhance AFM studies. I will show that CFS has enhanced force resolution and sensitivity than AFM, and thus, is a better force spectroscopy tool for single molecule measurements. I will show that one major contribution that CFS makes to knowledge available on mechanical properties of biomoleucles is the direct measurement of internal friction, as well as elasticity of these molecules. While elasticity resists conformational changes, internal friction limits the rate of these changes. The importance of internal friction property in biomoleucles such as proteins 
is in reducing the rate of folding and unfolding process during a biological activity. There is much known about friction on polymeric molecules mediated by solvent (i.e. solvent friction), but there is little known about internal friction of these molecules. CFS is capable of measuring the internal friction. This will be presented in future chapters.

\section{Overview of Single Molecule Force Spectroscopy Techniques}

Several techniques have been developed which differ in accessible force and frequency bandwidth [1, 8, 9]. (1) Magnetic tweezers (MT) utilize a magnetic field to manipulate the position of a superparamagnetic bead in order to stretch and/or twist the single molecule attached to the bead. They are used in measuring elasticity and structural transitions of DNA [1, 8, 9]. In this technique, forces in range of $0.01 \mathrm{pN}$ to $100 \mathrm{pN}$ can be exerted with a bandwidth of 10 to $100 \mathrm{~Hz}$. (2) Microneedles are used in investigations of tensile strength of a single actin filament [10]. The technique has a force range $>0.1 \mathrm{pN}$ and bandwidth $<10 \mathrm{~Hz}$. (3) The Biomembrane force probe is used in studies of molecular adhesion and structural linkage at biological interfaces [11]. This technique has a force range of 0.5 to $1000 \mathrm{pN}$ and bandwidth $<1 \mathrm{kHz}$. (4) In the optical tweezers (OT) technique a polymeric bead with the attached molecule of interest is trapped in a focused laser beam while the other end of the molecule is tethered to a surface or another bead. Optical tweezers are used in investigations of elastic properties and folding of nucleic acids, mechanisms of molecular motors motion, DNA and RNA polymerases and protein-DNA interactions [12-19]. In this technique, forces in range of 0.1 to $150 \mathrm{pN}$ can be exerted with bandwidth 1 to $10 \mathrm{kHz}$. (5) AFM is used to measure intramolecular structural transitions in macromolecules, receptor-ligand interactions, protein-protein interactions, interaction between complementary strands of DNA, measuring the viscoelastic properties of biological structures and interactions between cells [20-24]. The AFM can stretch biopolymers into important conformations that are inaccessible to other methods of measurement [24]. AFM has a force range of $>1 \mathrm{pN}$ and bandwidth $<100 \mathrm{kHz}$. Among these techniques, MT, OT and AFM are most commonly used. A comparison between these techniques as explained above is also shown in Table 1-1. Note that the chief disadvantage of AFM is the relatively poor force resolution and the most significant advantages are ease of use and the spatial resolution. Because my work uses AFM this review will focus on AFM-based single molecule force spectroscopy. 
Table 1-1: Comparison of techniques used for single molecule force spectroscopy $[1,8,9]$.

\begin{tabular}{|l|c|c|c|c|c|}
\hline Method & Force $(\mathrm{pN})$ & Distance $(\mathrm{nm})$ & Bandwidth $(\mathrm{Hz})$ & $\begin{array}{l}\text { Force resolution } \\
(\mathrm{pN})\end{array}$ & $\begin{array}{l}\text { Spatial resolution } \\
(\mathrm{nm})\end{array}$ \\
\hline MT & $0.01-100$ & $2-10$ & $10-100$ & 0.001 & $2-10$ \\
\hline OT & $0.1-150$ & $>1000$ & $1000-10000$ & 0.1 & $0.1-2$ \\
\hline AFM & $>1$ & $>1$ & 100000 & 10 & 0.1 \\
\hline
\end{tabular}

\section{Experiments Using Single molecule AFM}

One major area of research using single molecule AFM is intermolecular interactions. In particular, there are many studies of receptor-ligand type of interactions, e.g. biotin interacting with streptavidin or avidin. In their pioneering work, Moy et al. (1994) [25] investigated the dissociation pathways of avidin-biotin complex. They found an unbinding force of $\sim 160 \mathrm{pN}$ with an effective rupture length of 9.5 angstroms. Evans and Ritchie (1997) [26] argued that the unbinding phenomenon as a kinetic process should depend on the loading rate, and thus in order to obtain the energy landscape of intermolecular interactions, molecular adhesion forces must be examined over an enormous span of time scales, from $0.01 \mathrm{pN} \mathrm{s}^{-1}$ to $10 \mathrm{nN} \mathrm{s}^{-1}$. This loading rate dependency was shown by Merkel et al. in experimental measurements of avidin-biotin interaction forces [27]. Schlierf et al. [28] showed that similar loading rate dependency is also present in intramolecular interactions in experimental measurements of unfolding ubiquitin.

One focus of this thesis is measurement of DNA mechanical properties. Both the mechanical properties of individual strands and the binding between DNA molecules have been studied extensively by AFM. In their pioneering work, Lee et al. (1994) [22] used complementary strands of oligonucleotides that did not contain self-complementary regions to measure the force of base pairing. They found a force of $\sim 70 \mathrm{pN}$ per base pair. Boland and Ratner [29] measured a rupture force of $54 \mathrm{pN}$ for a single A-T pair. These measurements are however higher than 10 $\mathrm{pN}$ for a single A-T pair and $15 \mathrm{pN}$ for a single G-C pair reported in micro-needles experiment 
[30] or $9 \mathrm{pN}$ for a single A-T pair and $20 \mathrm{pN}$ for a single $\mathrm{G}-\mathrm{C}$ pair reported in AFM experiment by Rief et al. [31]. To resolve the discrepancy in these measurements recently Krautbauer et al. [32] structured repeating units of 10 or 20 A-T bases or G-C bases and measured the force to unzip the conjugated double stranded DNA (dsDNA). Supporting their experiments with thermodynamic equilibrium simulations, they reported an unzipping force between 10 and $20 \mathrm{pN}$ for 10 paired bases. The larger values reported in previous measurements can be due to nonspecific interactions between the force probe and surface. Reference to Table 1-1 shows the obvious problem that the magnitude of these forces is right at the limit of AFM resolution. Obviously there is lack of experimental data on unzipping short dsDNA ( $<100$ base pairs) at varying loading rates. These measurements are generally performed at moderate pulling velocities $\sim 100 \mathrm{~nm} \mathrm{~s}^{-1}$.

The AFM has proven particularly useful in studying forces acting on proteins. A major effort has focused on (un)folding kinetics of proteins [33] and to investigate their force-induced conformational transition [23,34]. Oberhauser et el. [35] applied varying force over the range of 0 to $300 \mathrm{pN}$ on $\mathrm{I} 27$ module of human cardiac titin and found that the module extends $\sim 22 \mathrm{~nm}$ when unfolded. This class of AFM measurements is called static force-elongation measurements.

The principal limitation on the force resolution in AFM is the thermal noise that is intrinsic to any mechanical system: the AFM cantilever vibrates with an average energy equal to one half of the thermal energy. Recognizing this problem, Kawakami et al. [36] developed a modification of the basic AFM experiment in which they exploited the thermal vibration. They measured the thermally-stimulated power spectral density (PSD) of one AFM cantilever rather than static deflection. In their experiments, dextran was initially stretched to a set force, and then relaxed to various extensions with lower forces. At each extension, the cantilever dwelled for a few seconds while its PSD was measured. The polymer was then fully stretched again and detached from the surface. They measured the polymer stiffness and friction at various polymer extensions using the PSD data and found very good agreement with pulling in the static (force-extension) mode. In another study, Bippes et al. [37] investigated the viscoelastic properties of dextran while stretching a single chain of the molecule and collecting the thermal fluctuations of the 
cantilever. They report a good agreement between their results and results of Kawakami et al. [36]. As part of device evaluation and preliminary results, I also studied dextran molecule and found qualitative agreement in mechanical properties of this molecule with previous studies. These experiments belong to a different class of AFM measurement that is called dynamic force spectroscopy. In addition to using the thermal fluctuations of the cantilever as stimuli [38], it is possible to derive the cantilever using magnetically driven oscillations [38-40]. The main advantage of dynamic mode to static mode is measurement of the chain friction that is inaccessible to the static measurement.

\section{Limitations of AFM-based single molecule force spectroscopy}

AFM-based single molecule force spectroscopy has gained popularity due to its wide force and frequency bandwidth, ease of use, and reproducibility of measurements from different laboratories [41]. But one major limitation of the technique is noise on force detection. The sources of noise are $i$. Brownian forces due to interaction with solvent molecules, $i i$. the resulting thermal fluctuations of the cantilever (i.e. thermal noise) that sets the highest noise force limit on detection of molecular events, and iii. large hydrodynamic interaction with solid surface when the cantilever is placed in proximity of the surface to pull a molecule. These noise forces can be shown in an equation of motion for a cantilever of mass $m$ and stiffness $k_{\mathrm{c}}$ using Newton's second law:

$$
m \ddot{x}=-\gamma_{\mathrm{a}} \dot{x}-k_{\mathrm{c}} x+f_{n},
$$

where $f_{n}$ is the Brownian force. $x$ is the thermal fluctuations of the cantilever, and $\dot{x}$ and $\ddot{x}$ are the velocity and acceleration of the cantilever. $\gamma_{\mathrm{a}}$ is the viscous friction, and thus $\gamma_{\mathrm{a}} \dot{x}$ denotes hydrodynamic force on the cantilever. The thermal noise $\left\langle x^{2}\right\rangle(\langle\cdots\rangle$ is ensemble average $)$ is inescapable: at any given temperature, all occupied vibrational modes of the cantilever have energy proportional to the temperature. This vibration obscures "good signals" that are also deflections of the cantilever. One solution to this problem is to measure slowly (or average the signal) such that the fluctuations average out. The fluctuations have greatest amplitude near the resonant frequency, so the averaging frequency must be below the cantilever resonant frequency. Such a solution limits the bandwidth of the measurement and obscures dynamic data. 


\section{My thesis is directed toward lowering the limit of thermal noise by measuring the} correlations in thermal motions of two cantilevers. Correlation measurement between two cantilevers, in addition, results in reduced correlated Brownian forces (case $i$ above) and hydrodynamic forces between the two cantilevers (case iii above). The idea for studying correlations has been previously employed in dual trap optical tweezers measurements [12] and was theoretically described in Paul and Cross [42]. Acoustic noise, mechanical noise and optical noise which are independent of one probe to the other do not show up in the correlation signal.

From the equipartition theorem, the mean square thermal motions of an object in a harmonic potential of stiffness $k_{\mathrm{c}}$ is [8]:

$$
\left\langle x^{2}\right\rangle=\frac{k_{\mathrm{B}} T}{k_{\mathrm{c}}}
$$

where $x$ is in the direction of stiffness $k_{\mathrm{c}}$. The square root $\sqrt{\left\langle x^{2}\right\rangle}$ denotes the average amplitude of thermal motion (noise) of the object. The limit of force resolution due to thermal noise is then:

$$
\Delta F_{x}=k_{\mathrm{c}} \sqrt{\left\langle x^{2}\right\rangle}=\sqrt{k_{\mathrm{c}} k_{\mathrm{B}} T} .
$$

I predict that cross-correlation of two cantilevers:

$$
\left\langle x_{1}(0) x_{2}(t)\right\rangle,
$$

is smaller than the autocorrelation of one cantilever (correlation of one cantilever with itself):

$$
\left\langle x_{1}(0) x_{1}(t)\right\rangle
$$

where $x_{1}$ is the thermal motions of the first cantilevers, $x_{2}$ is the thermal motions of the second cantilever, and $t$ is the time lag in correlation measurement. The maximum of the autocorrelation function (1.5) is at $t=0$ that is shown in equation (1.2). The maximum of the cross-correlation function (1.4):

$$
\max \left(\left\langle x_{1}(0) x_{2}(t)\right\rangle\right),
$$

can give an estimate of the force resolution in cross-correlation of two cantilevers due to thermal noise [43]: 


$$
\Delta F_{12}=k_{\mathrm{c}} \max \left(\sqrt{\left\langle x_{1}(0) x_{2}(t)\right\rangle}\right)
$$

and can be obtained and compared with equation (1.3). In equations (1.6) and (1.7), the maximum of the cross-correlation function is not necessarily at zero time lag; however, the maximum of the autocorrelation function is by definition at zero time lag (set $t=0$ in equation (1.5) to get equation (1.2)). Smaller cross-correlation function is intuitive and should be distance dependent. Correlation in thermal motions of the two cantilevers arises from fluid coupling the two cantilevers. This coupling is a small effect, resulting in a small cross-correlation function compared with the autocorrelation of one cantilever with itself. This is the subject of Chapter 2 and start of Chapter 6. In Chapter 2, I will compare the power spectral density (i.e. Fourier transform) of cross-correlation function and autocorrelation function at all frequencies around the fundamental resonance frequency of the cantilevers. I show that the cross-correlation power spectral density in the absence of a tethered molecule has much smaller noise amplitude than the autocorrelation power spectral density. In Chapter 6, I will compare the cross-correlation and autocorrelation functions in time domain (i.e. equations (1.4) and (1.5), respectively). I will show that the maximum of cross-correlation function is smaller than the maximum of autocorrelation function, and that the cross-correlation function decreases monotonically with separation between the two cantilevers. The frequency space comparison (between the power spectral densities) is shown earlier in this thesis since it is important to show that at all frequencies around the fundamental resonance frequency of the cantilevers the noise is much smaller when the thermal motions of two cantilevers are correlated. After tethering a molecule between the two tips, there is a larger percentage variation in the cross-correlation compared to the autocorrelation, giving a better force sensitivity.

From the fluctuation-dissipation theorem, the force resolution due to Brownian forces is [43, 44]:

$$
\Delta F_{n}=\sqrt{4 \gamma_{\mathrm{a}} k_{\mathrm{B}} T \mathrm{BW}},
$$

where $\mathrm{BW}$ is the frequency bandwidth of data acquisition, and $\gamma_{\mathrm{a}}$ is the viscous friction of the probe. Equation (1.8) is valid for frequency bandwidth $\mathrm{BW} \ll \omega_{\text {roll-off }}$ where the roll-off frequency $\omega_{\text {roll-off }}=k_{\mathrm{c}} / \gamma_{\mathrm{a}}$. For a typical cantilever $k_{\mathrm{c}} \sim 10^{-1} \mathrm{~N} / \mathrm{m}$ and $\gamma_{\mathrm{a}} \sim 10^{-6} \mathrm{~kg} / \mathrm{s}$; this gives $\omega_{\text {roll-off }} \sim 10^{6} \mathrm{rad} / \mathrm{s}$. The fundamental resonance frequency in solvent of almost all cantilevers 
used in single molecule measurements is much smaller than the roll-off frequency [36, 39], and thus equation (1.8) is a valid presentation of Brownian noise force in AFM measurements. From equation (1.8), reducing BW enhances the force resolution set by Brownian force. As a matter of fact, reducing BW will also significantly reduce the noise due to thermal fluctuations of the cantilever. Lowering BW is normally done in static AFM measurements applying a low-pass filter on fast fluctuations data. However, this approach is not practical when fast fluctuations are required for dynamic measurements.

It is thus more practical to reduce the viscous friction $\gamma_{\mathrm{a}}$. This can be done by for example reducing the dimensions of the probe, or using less viscous fluid. However, the viscous friction increases drastically when the cantilever is placed in proximity of a surface in single molecule measurements using AFM. In the case of CFS, viscous friction reduction is obtained by having a much smaller area of hydrodynamic interaction between the two cantilevers comparing to the entire cantilever body as in single cantilever measurements (see Figure 1-1). In this case $\gamma_{c}$ (viscous friction from the fluid in the gap of the two cantilevers) is the viscous friction. Therefore, I predict that $\gamma_{\mathrm{c}}<\gamma_{\mathrm{a}}$ at all separations and thus CFS has smaller noise force limit due to Brownian forces than AFM. This is discussed in Chapter 2. The reduction in viscous friction automatically reduces the background hydrodynamic interaction force. 

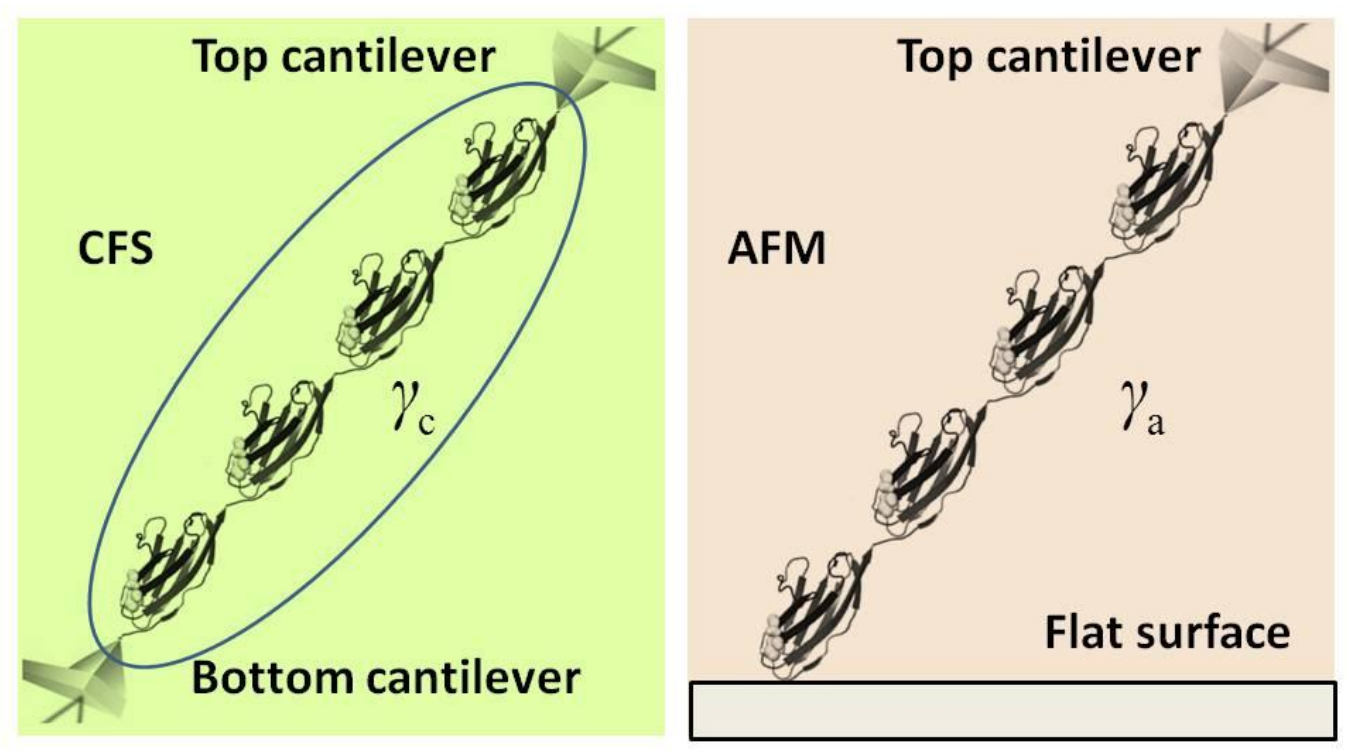

Figure 1-1: Schematic of AFM with viscous friction $\gamma_{\mathrm{a}}$ and CFS with viscous friction $\gamma_{\mathrm{c}}$. $\gamma_{\mathrm{c}} \ll \gamma_{\mathrm{a}}$ due to much smaller area of inter-cantilever hydrodynamic interaction. This results in much better force resolution in CFS than AFM.

Correlations in the thermal noise are also interesting. It is important to note that the frequency distribution of thermal noise is affected by interactions with the environment. For example, a highly viscous fluid environment of the cantilever leads to a broadening of the frequency distribution of the thermal noise of the cantilevers. Thus, in single-cantilever measurements, the thermal noise is modulated by the fluid that immerses the entire cantilever. This effect has been exploited to measure the properties of fluids [45].

The important point for this thesis is that the correlation between the thermal motions of two cantilevers is dominated only by the material that interacts with both cantilevers, i.e. the material between the tips. And the noise floor is not the fluctuations on the entire cantilever, it is the noise on the correlations between the cantilevers, which does not have a theoretical lower limit to its integral.

The lack of a fundamental noise floor is particularly important in biomolecular studies because many biomolecular events have energy similar to the thermal energy. For a cantilever of spring constant $k_{\mathrm{c}}=0.1 \mathrm{~N} / \mathrm{m}$ the thermal noise on a single cantilever sets a bound on the noise force 
amplitude of order $\sqrt{k_{\mathrm{c}} k_{\mathrm{B}} T} \cong 20 \mathrm{pN}$ from the equipartition theorem. As described about in the previous section $20 \mathrm{pN}$ is larger than the force required to rupture short DNA base pairs. Thus a useful development for the field of AFM molecule spectroscopy would be to move from the analysis of single-cantilever to the analysis of the correlations between two cantilevers. As a precursor to such single molecule studies, it is necessary to understand the correlations of the two cantilevers in fluid without the connecting single molecule. This is the subject of Chapters 4,5 and 6 where different configurations for the dual cantilevers is designed and tested. Such a development, in addition, opens a platform for a new micro-rheology technique [46].

In a typical force-elongation experiment in AFM, polymer extension (PE) can be obtained from:

$$
P E=\Delta y+\Delta x,
$$

where $\Delta y$ is the z-piezo displacement (attached to the cantilever base) and $\Delta x$ is the low-pass filtered deflection of the cantilever. When the cantilever is deflected downward (for example due to polymer force on the cantilever) $\Delta x$ is a negative value ${ }^{2}$. Thus, $\Delta x=-F / k_{\mathrm{c}}$ where the force exerted by the polymer, $F$, is assumed to be a positive number. The polymer static stiffness at each point on its elongation or extension curve can be obtained from:

$$
k_{p}=\frac{\delta F}{\delta P E}=\frac{\delta F}{\delta\left(\Delta y-F / k_{\mathrm{c}}\right)} .
$$

$\delta F$ thus must be larger than the force sensitivity limit of the AFM ( $\left.\delta F>\Delta F_{\text {ms }}\right)$ to be measurable by the AFM.

Using two cantilevers in the force-elongation mode of CFS modifies equation (1.9):

$$
P E=\Delta y+\Delta x_{1}-\Delta x_{2},
$$

where $\Delta x_{1}$ and $\Delta x_{2}$ are the deflections of the top and bottom cantilevers respectively. Under a polymer force $F$, the top cantilever deflects downward resulting in experimentally negative value, while the upward deflection of the bottom cantilever results in experimentally positive value. Thus, $\Delta x_{1}=-F / k_{\mathrm{c}, \mathrm{t}}$ and $\Delta x_{2}=F / k_{\mathrm{c}, \mathrm{b}}$, where $k_{\mathrm{c}, \mathrm{t}}$ and $k_{\mathrm{c}, \mathrm{b}}$ are respectively the spring

\footnotetext{
${ }^{2}$ This is the convention used in this thesis and kept for consistency.
} 
constants of the top and bottom cantilevers and $F$ is assumed to be a positive number. Equation (1.10) forms:

$$
k_{p}=\frac{\delta F}{\delta P E}=\frac{\delta F}{\delta(\Delta y)-\delta F\left(1 / k_{\mathrm{c}, \mathrm{t}}+1 / k_{\mathrm{c}, \mathrm{b}}\right)} .
$$

Equation (1.12) shows that CFS can also be used as a static force-elongation tool in single molecule force spectroscopy but with slightly better force resolution, due to the diminished stiffness of the compound spring. The main application of CFS is in dynamic measurements.

\section{Summary}

The CFS measurements are predicted to have the following advantages over (conventional) analysis of a single cantilever in AFM:

- decreased damping due to a smaller hydrodynamic interaction between two cantilevers than over an entire cantilever,

- a smaller magnitude of correlation function, and a larger relative change in the correlation function when a polymer is straddled between two tips.

These effects are discussed in the future chapters. 


\section{Correlation Force Spectroscopy}

\section{Correlation Force Spectroscopy: Rationale}

In principle, the main advantage of CFS over one cantilever AFM is that the thermal noise in the cross-correlation between two cantilevers is much smaller than the thermal noise in the autocorrelation for a single cantilever [43, 47], so here I examine this prediction. The noise spectrum of a single cantilever and the cross-correlation noise spectrum of two cantilevers are shown in Figure 2-1. It is clear that the cross-correlation noise spectrum has a much smaller magnitude and is typically about $1 / 4$ of the autocorrelation noise spectrum (i.e. thermal noise of a single cantilever) for the same cantilever in the same fluid. This is the first advantage of CFS over AFM: Upon tethering a molecule between the two tips, variation of the smaller crosscorrelation noise spectrum due to the molecule force (or molecule mechanical properties: stiffness and friction) is larger comparing to the larger autocorrelation noise spectrum. This means a better sensitivity for single molecule measurements. It is noted that in single molecule force spectroscopy, this noise from the solvent coupling of the cantilevers will be the "noise" that sets the limit of thermal force resolution. Thus, by changing from one cantilever to two cantilever measurements, a fundamental noise limit in AFM single molecule force spectroscopy is lowered. The dispersion of noise in the cross-correlation also has the interesting feature that there is a particular frequency near the resonant frequency where the thermal noise is zero (see Figure 2-1). With respect to equations (1.2) and (1.6), the area under the autocorrelation power spectral density is equal to $\left\langle x^{2}\right\rangle^{1 / 2}$ and the absolute area under the cross-correlation power spectral density corresponds to $\max \left(\sqrt{\left\langle x_{1}(t) x_{2}(0)\right\rangle}\right)$. It is clear from the figure that CFS has superior force resolution compared to AFM (see equations (1.3) and (1.7)). 


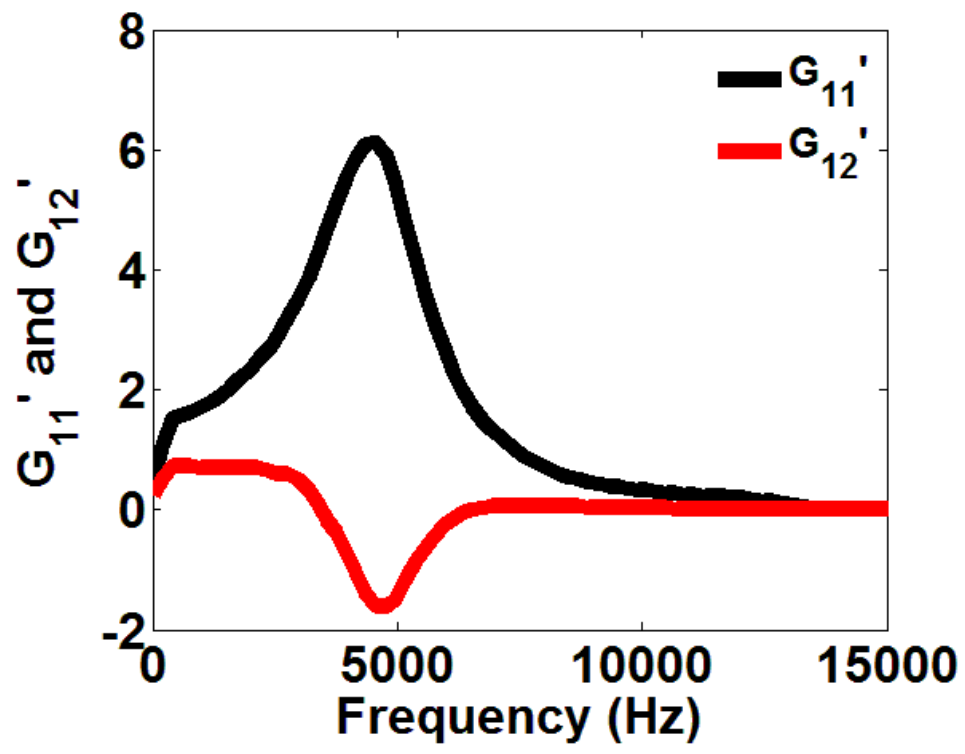

Figure 2-1: Comparison between the normalized autocorrelation noise spectrum $\left(G_{11}^{\prime}\right)$ and the normalized cross-correlation noise spectrum $\left(G_{12}^{\prime}\right)$ in water at $23^{\circ} \mathrm{C}$. Cantilevers used are ORC8B: length $=200 \mu \mathrm{m}$, width $=40 \mu \mathrm{m}, \mathrm{k}=0.1 \mathrm{Nm}^{-1}$. The separation between the cantilevers is $d=$ $6.5 \mu \mathrm{m}(s=318 \mathrm{~nm})$ in the vertically offset CFS as shown in Figure 2-4-b.

Fitted values of $\gamma_{\mathrm{c}}$ and $\gamma_{\mathrm{a}}$ (see Figure 2-2) show that $\gamma_{\mathrm{c}}$ is about an order of magnitude smaller than $\gamma_{\mathrm{a}}$ for the distance range $<1 \mu \mathrm{m}$, which is the applicable range of single molecule force measurements. From equation (1.8) the Brownian force resolution scales with the root of the viscous friction, so the second advantage of CFS over AFM is the reduced Brownian noise force owing to fluid coupling against which all other coupling (e.g. that of a straddling molecule) must be measured. Later I show that the relative changes in coupling are greater for CFS than conventional AFM when a molecule is tethered between the tips. Reduced viscous friction in CFS compared to AFM directly means reduced hydrodynamic interaction which also sets a background noise force. 


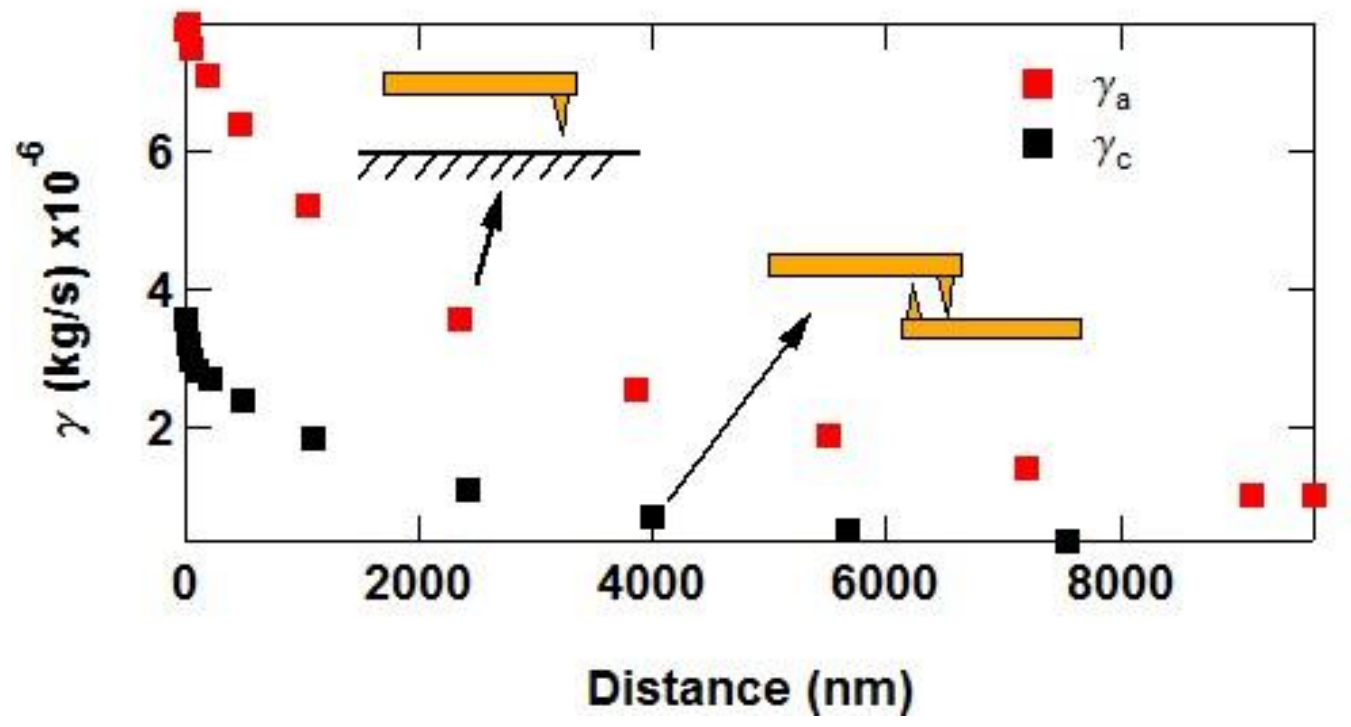

Figure 2-2: Experimental measurements of $\gamma_{\mathrm{a}}$, the viscous friction on a single cantilever and $\gamma_{\mathrm{c}}$, the viscous friction between two closely spaced cantilevers, as a function of distance. $\gamma_{c}$ is much smaller.

Having the idea of correlating thermal motions of two cantilevers in mind, different configurations for the two cantilevers can be anticipated. The simplest ones are the anti-parallel laterally offset and anti-parallel vertically offset configurations. Each of these configurations has its advantages and applications that will be discussed in later sections. So, in the remained of this document, I will discuss the analysis of the two cantilever technique, the development of the laterally offset (see Figure 2-3) and vertically offset (see Figure 2-4) device, results on rheological measurements, and results on single molecule force spectroscopy and then future work. Significant portions of this thesis detail equipment development.

\section{Correlation Force Spectroscopy: Development}

CFS consists of a pair of micrometer-scale cantilevers that are closely spaced (see Figure 2-3). Each cantilever undergoes thermal fluctuations due to interaction with the fluid molecules. However the thermal fluctuations of the cantilevers are not independent, but coupled through the fluid spanning the gap of the two cantilevers. By studying the correlated fluctuations of the cantilevers, properties of the intervening material can be obtained. Detection of the thermal 
fluctuations of the cantilevers is done using optical lever technique using individual lasers and individual photodetectors for each cantilever. Precautionary measurements should be taken to cancel cross-talk between the two lasers in the detection systems, for example by using appropriate optical filters in front of photodetectors or spatial adjustments of the lasers and photodetectors. I use the absence of a cross-correlation at zero time lag as a requirement for no instrumental cross-talk between the signals. It is important that the signals are recorded synchronously, because any phase lag between the two detection systems will produce artifacts. This check can be done by shining both lasers on one cantilever and calculating the autocorrelation (of one of the laser signals) and cross-correlation (of the two laser signals). A similar auto- and cross-correlation function in this case justifies no phase lag between the two detection signals. Another important parameter is thermal drift of components due to temperature fluctuations in the laboratory. While there is no way to cancel this effect, it is possible to minimize it by taking appropriate measures in each CFS configuration, which are discussed later. By measurement of the power spectral density as a function of laser power, I showed that the laser had a negligible effect on the temperature of the cantilever. The other important parameter is the gap of the two cantilevers. By increasing the gap, the two cantilevers are less coupled thus the correlation in thermal fluctuations is weaker, i.e. the cross-correlation noise spectrum is weaker. 


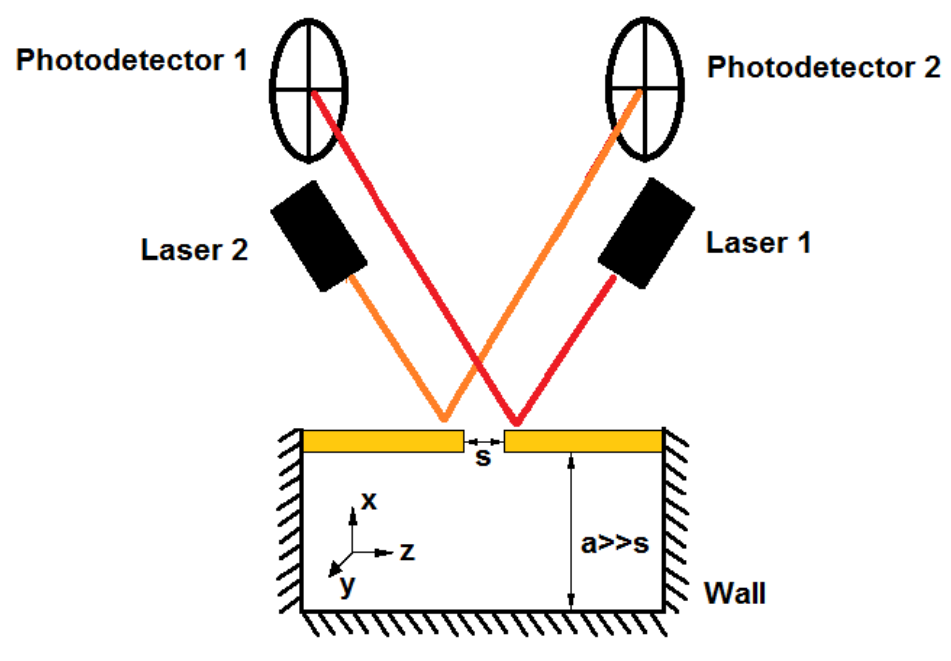

(a)
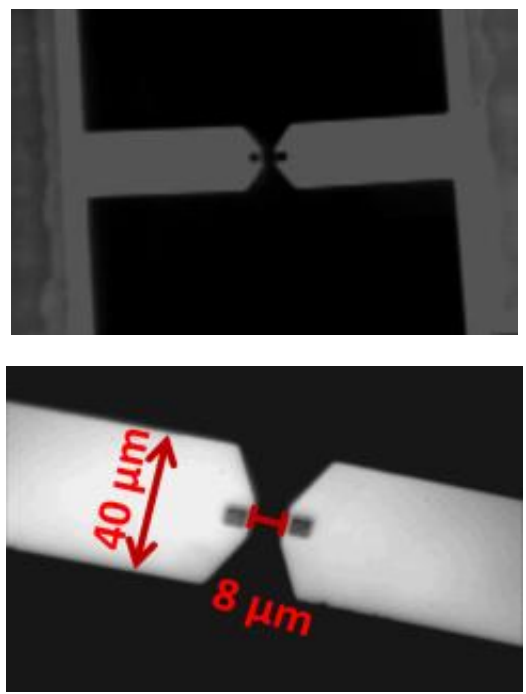

(b)

Figure 2-3: (a) Schematic of the cantilevers and detection system in the antiparallel laterally offset CFS. (b) Light microscope images of closely-spaced AFM cantilevers seen from above. The cantilevers are $100 \mu \mathrm{m}$ long and $40 \mu \mathrm{m}$ wide. The lateral separation between the cantilevers is $8 \mu \mathrm{m}$.

\section{Laterally Offset Configuration}

In the laterally offset CFS, a pair of commercial AFM cantilevers are mounted in an antiparallel configuration between two glass slides as shown schematically in Figure 2-3-a. The cantilevers are positioned under an optical microscope and then glued in place (see Figure 2-3-b).

The thermal drift is minimized in laterally offset CFS because of small distances and material matching: the two cantilevers are joined by the cantilever chip $\left(\sim 3 \times 1 \mathrm{~mm}^{2}\right.$ of silicon nitride), a thin layer of glue, a glass slide, and a second cantilever chip. The cantilevers are mounted > 1 $\mathrm{mm}$ away from the glass slide, so there is minimal fluid coupling between the cantilever and rigid glass wall. In the vertical direction (Figure 2-3-a) all materials are matched for the left and right cantilevers, and in the horizontal direction, the bases are separated by only about $400 \mu \mathrm{m}$ of glass, which is a much smaller connection than between tip and sample in a commercial AFM. There are no "moving" parts; the only motion is molecular motion of the fluid and the fluctuating deflection of the cantilevers due to interaction with the fluid at equilibrium. 
The deflection of each of the cantilevers is measured by two lasers (Schäfter + Kirchhoff GmbH, Hamburg, Germany) reflected by the cantilever onto a position sensitive diode (Pacific Silicon Sensor, CA). In this case I use a different wavelength of laser (635 and $680 \mathrm{~nm}$ ) for each cantilever, and (in some experiments) use a wavelength filter over each diode and different light paths to prevent cross-talk between the signals. The signals are recorded synchronously by an Asylum Research Atomic Force Microscope controller (Nyquist frequency, $f_{\mathrm{Ny}}=25 \mathrm{kHz}$ ) for type B cantilevers and a National Instrument (Irvine, CA) PCI-6110 Data Acquisition card ( $f_{\mathrm{Ny}}=$ $500 \mathrm{kHz}$ ) for type A cantilevers.

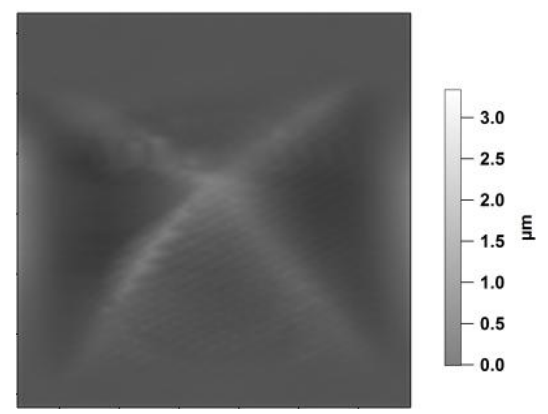

(a)

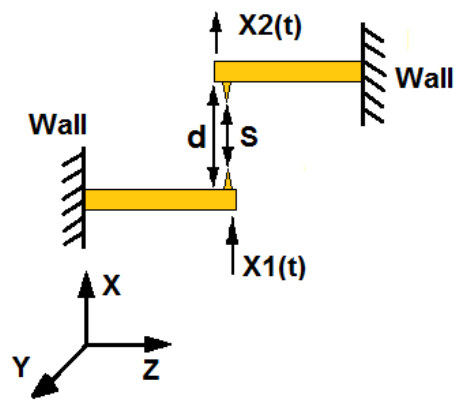

(b)

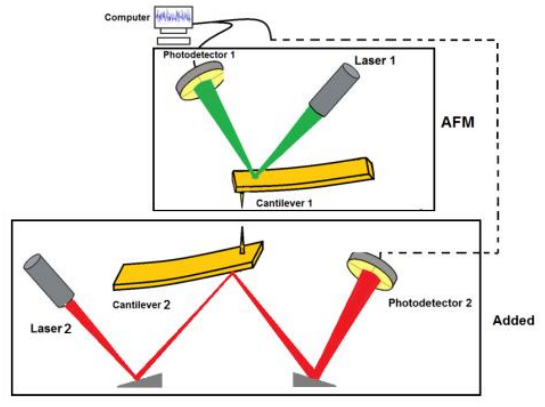

(c)

Figure 2-4: (a) Atomic force microscopy contact mode image in water of the bottom cantilever tip obtained by scanning the bottom cantilever and measuring the height signal. This imaging is used to align the tips. (b) Schematic of antiparallel vertically offset cantilevers. (c) Schematic of the cantilevers and detection system in the antiparallel vertically offset CFS.

\section{Vertically Offset Configuration}

The vertically offset CFS consists of a pair of commercial AFM cantilevers mounted in an antiparallel vertically offset configuration as shown schematically in Figure 2-4-b. The top cantilever is mounted in a commercial AFM (Asylum Research, MFP3D-bio, CA) that has the full functionality of AFM, such as sub-nm resolution in deflection and displacement in three dimensions. A home-built cantilever mount and optical lever cantilever deflection was incorporated into the AFM in place of "the sample" (see also Figure 2-4-c). For this additional cantilever deflection sensor, a laser (Schäfter + Kirchhoff GmbH, Hamburg, Germany, 
51nanoFCM) is steered by a mirror onto the cantilever and the reflection off the cantilever falls onto a second mirror which then steers the beam onto a split photodiode (Pacific Silicon Sensor, CA, QP50-6-18u-SD2). A different wavelength $(680 \mathrm{~nm})$ of laser was used for the bottom cantilever compared to the top cantilever (Superluminescent diode with an $860 \mathrm{~nm}$ wavelength) to allow frequency filtering, thereby preventing optical contamination between the two signals. The two laser beams are also physically separated so that they do not contaminate each other. The main danger of unwanted cross-correlation (that does not arise from the fluid) is from light from a single laser that ends up striking both cantilevers and entering a single diode. This could occur if the cantilevers are parallel and if the laser beam is larger than the cantilever or the cantilever allows transmission of light. I try to cover all these possibilities by using standard gold coatings on the cantilevers, by focusing the laser beams to a spot size that is smaller than the cantilever, and by not making the cantilevers perfectly parallel.

The experimental procedure is as follows. A fluid droplet is injected and held in place by capillary force and the system is left to reach equilibrium for about 40 minutes. Initially, while the cantilevers are at large separation, the PSD of each cantilever is measured and a simple harmonic oscillator fit is applied to PSDs to yield the resonant frequency and quality factor of each cantilever at infinite separation. The PSD in voltage units is given by:

$$
\operatorname{PSD}(\omega)=(\text { Inv. OLS })^{-2} \times\left[\left(\frac{4 k_{B} T}{k_{\mathrm{c}}} \frac{\left(\frac{2 \pi \omega_{r}}{Q}\right)}{\left(\left(\frac{\omega}{\omega_{r}}\right)^{2}-1\right)^{2}+\left(\frac{2 \pi \omega}{Q}\right)^{2}}\right]^{1 / 2}+C\right]^{2},
$$

where $Q$ is the quality factor, $C$ is the white noise, and Inv. OLS is the inverse optical lever sensitivity $(\mathrm{nm} / \mathrm{v})$.

To determine the optical lever sensitivity and thereby spring constant, the top cantilever is brought into contact with a hard flat surface on the bottom cantilever mount and a single forceextension measurement is performed. This is done by translating the clamped end of the top cantilever downward a known distance with the z-piezo, and measuring the uncalibrated deflection of the cantilever in units of volts. When the tip of the top cantilever is in contact with 
the flat surface, the deflection in nanometers is equal to the distance travelled by the z-piezo. Inv. OLS of the top cantilever is then the slope of the deflection-z-piezo plot in the contact region. Knowing the Inv. OLS of the top cantilever and fitting the PSD of the top cantilever to equation (2.1) the spring constant of the top cantilever can be calculated. The calibration of the bottom cantilever can be performed in the same manner by pressing against the bottom surface of the top cantilever chip. Alternatively, for the bottom cantilever, initially the two cantilevers are pressed against each other by translating the clamped end of the top cantilever downward a known distance with the z-piezo. In the contact region, the force between the two cantilevers is the same. Thus, by knowing Inv. OLS and spring constant of the top cantilever, spring constant of the bottom cantilever can be calculated (see equations (2.2) and (2.3) below). Finally, fitting PSD of the bottom cantilever to equation (2.1), its Inv. OLS can be calculated.

$$
\begin{gathered}
\Delta y=\left|\Delta x_{1}\right|+\left|\Delta x_{2}\right|, \\
F_{1}=F_{2} \Rightarrow k_{2}=\frac{k_{1} \times\left|\Delta x_{1}\right|}{\Delta y-\left|\Delta x_{1}\right|} .
\end{gathered}
$$

The tips are then brought near each other using the course z-control in the AFM (see Figure 2-5), and then imaging the bottom cantilever with the top cantilever to find the bottom tip. Note that the entire detection system of the bottom cantilever (cantilever, laser and diode) is scanned to maintain the alignment of the detection system on the cantilever. The Asylum AFM has a convenient "go there" function where the sample can be moved automatically to a location within a previously collected image; this function is used to move the top AFM tip to the apex of the bottom tip and into alignment. Periodically a new image can be taken to realign the horizontal position of the tips and re-establish the vertical separation zero position between the two tips. This is necessary to account for any drift in position. Once this is done, the z-piezo can be used to establish the required vertical separation. If used in distance clamp mode where the separation is kept constant, from this point and during data collection there are no "moving" parts; the only motion is molecular motion of the fluid and the fluctuating deflection of the cantilevers. However, if used in force clamp mode where the force on the top cantilever is kept constant, in addition to the molecular motion of the fluid and the fluctuating deflection of the cantilevers, z-piezo can also move to keep the constant force. Force clamp mode can only be used when a molecule is clamped between the two cantilevers. Otherwise, if used in the force 
extension mode, the top cantilever is retracted from the bottom cantilever at a constant velocity while fluctuations of both cantilevers are measured.
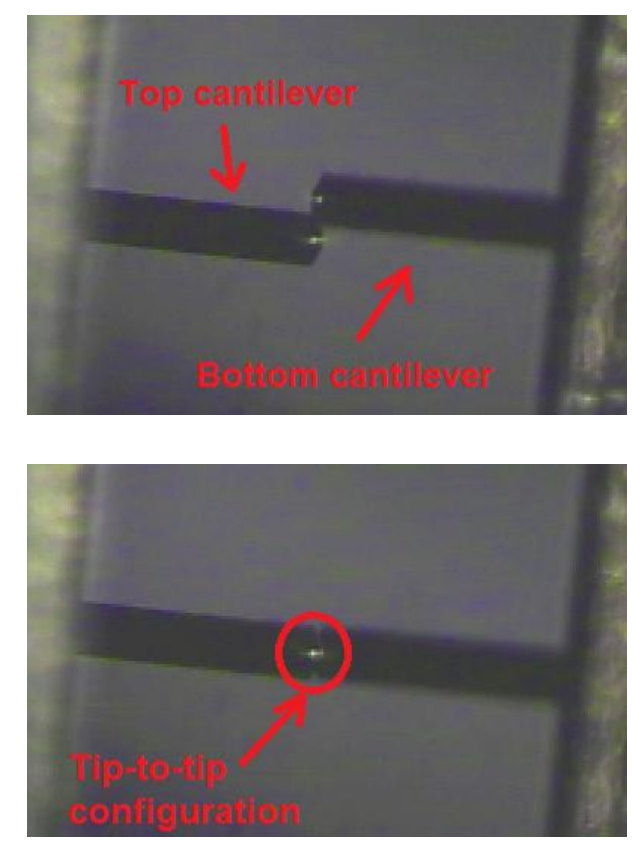

Figure 2-5: Top view optical microscope images of the cantilever pair. (Top) The two cantilevers are brought in proximity of each other. (Bottom) The two cantilevers are aligned coarsely using micrometer translation stage and then with nanometer precision using the piezoelectric devices of the AFM.

The signals from the top cantilever (part of the commercial AFM) and the bottom cantilever are recorded synchronously by an Asylum Research Atomic Force Microscope controller $\left(f_{\mathrm{Ny}}=25\right.$ $\mathrm{kHz}$ ) or a National Instrument (Irvine, CA) PCI-6110 Data Acquisition card $\left(f_{\mathrm{Ny}}=500 \mathrm{kHz}\right)$. Alternatively, the vertical offset experiments can be performed in a closed fluid cell with active temperature and solution control. The details of the apparatus are similar to that explained above. In this case the bottom cantilever is glued on the glass disk of the fluid cell which provides a path for the laser light to enter and the reflection to exit. The reflection is then steered on to a different photodiode using lens and mirror. The bottom detection system is mounted underneath the AFM (Nikon TE2000-U, Japan). 
There are a few differences between the laterally offset CFS and vertically offset CFS: (1) thermal drift is much lower in the former due to material matching and the way that the two levers are mounted, so that drifts are in the same direction; while thermal drift in vertical direction in the vertically offset CFS is a major drawback of the system; (2) the lateral offset device shears tethering molecules, whereas the vertical offset places them under tensioncompression. The latter is usually of interest in single molecule force spectroscopy experiments; (3) analysis of deflection data requires calibration of the sensitivity of the optical lever, which is usually done by pressing against a solid flat plate. The OLS depends on the alignment of the laser and the calibration must be done in each experiment, and sometimes must be redone during an experiment, if the alignment changes. The difficulty with the lateral offset experiment is that that there is no piezo in the system to press the cantilevers against a flat plate. Thus deflection data are normalized by the area under PSD. While the same approach can be adopted for vertically offset experiments, presence of z-piezo and flat surfaces makes possible direct measurement of OLS.

\section{Analysis of Correlations between Two Cantilevers}

The implementation of correlation measurements requires a method of analysis to obtain properties of the polymer molecule from the spectrum of deflection fluctuations. As it turns out, these fluctuations are very simply related to the ringdown of a cantilever from an initial displacement, which is itself rather straight-forward to model. The simplicity of this modeling is an advantage of CFS. The fluctuation-dissipation theorem [48] states that the manner in which a system returns from a linear macroscopic perturbation is related to time correlations of equilibrium microscopic fluctuations. Paul and Cross (2004) showed that the stochastic dynamics of pairs of small cantilevers in fluid could be analyzed in terms of deterministic behavior of a pair of cantilevers when cantilever 1 is subject to removal of a force, $F_{1}[42]$ :

$$
\begin{aligned}
& \left\langle x_{1}(0) x_{1}(t)\right\rangle=\frac{k_{\mathrm{B}} T}{F_{1}} X_{1}(t), \\
& \left\langle x_{1}(0) x_{2}(t)\right\rangle=\frac{k_{\mathrm{B}} T}{F_{1}} X_{2}(t) .
\end{aligned}
$$




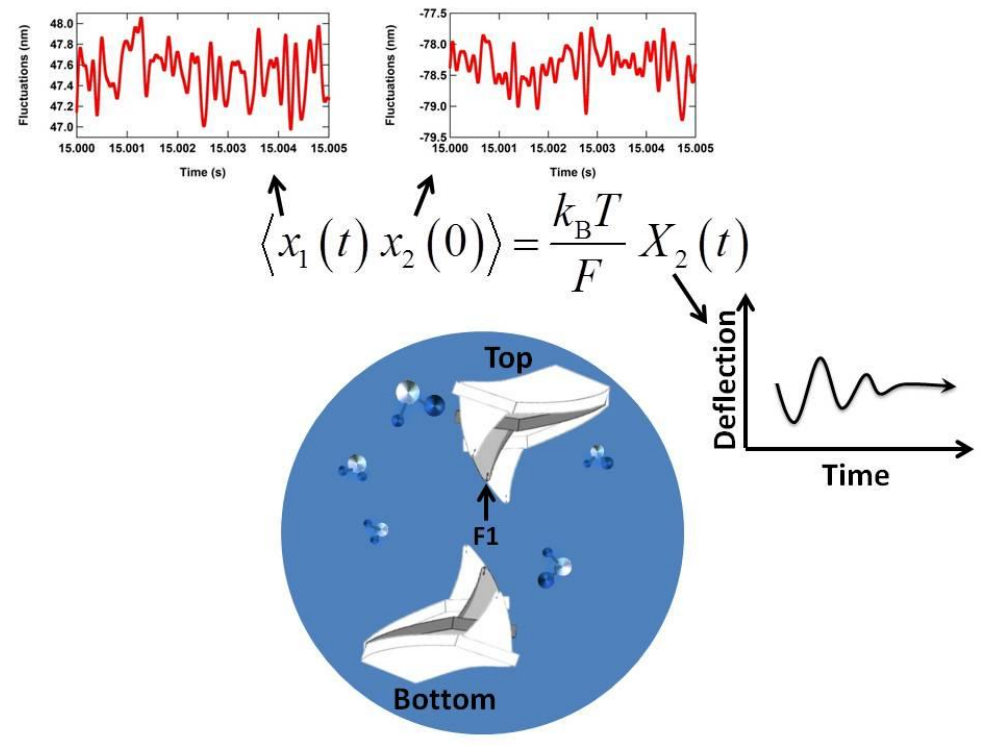

Figure 2-6: Schematic representation of equation (2.5). $x_{1}$ and $x_{2}$ are the thermal fluctuations of the top and bottom cantilever respectively and $X_{2}$ is the deterministic deflection of the bottom cantilever after removal of force $F_{1}$ from the top cantilever.

Figure 2-6 shows schematic representation of equation (2.5). Equation (2.4) relates the autocorrelation of stochastic fluctuations of a single cantilever, $\left\langle x_{1}(0) x_{1}(t)\right\rangle$, to its deterministic ringdown, $X_{1}(t)$, after removal of a step force, $F_{1}$, at time zero. $t$ is the time and $x$ is the deflection, which changes with time due to fluctuations. Equation (2.4) is the fluctuationdissipation theory for a single cantilever. Equation (2.5) relates the cross-correlation of stochastic fluctuations between the two cantilevers, $\left\langle x_{1}(0) x_{2}(t)\right\rangle$, to the deterministic response of the bottom cantilever, $X_{2}(t)$, as result of ringdown of the top cantilever after removal of a step force $F_{1}$, at time zero. In practice, the correlations, $\left\langle x_{1}(0) x_{1}(t)\right\rangle$ and $\left\langle x_{1}(0) x_{2}(t)\right\rangle$, are measured experimentally, and $X_{1}(t)$ and $X_{2}(t)$ are modeled either from finite element (FE) analysis [43], or from an equation of motion for the entire cantilever, such as a simple harmonic oscillator model. 


\section{Validation of Fluctuation-Dissipation Theorem for One Cantilever}

This thesis work requires the use of equations (2.4) and (2.5), which had not been validated experimentally. So I have measured the left and right side of equation (2.4) experimentally in the same system to test its validity: that is, the ringdown of a cantilever subject to a step force, and the fluctuations of the same cantilever are both measured in water. The step force was applied to the cantilever by first putting epoxy glue on a glass surface, then touching the epoxy with an AFM tip and then retracting the tip. The adhesion between the tip and the glue keeps the tip in contact with the glue, but when the adhesion force is exceeded by the spring force, the cantilever breaks free. The very short range of the adhesion force $(<1 \mathrm{~nm})$ means that when we pull the tip from the solid, the force drops very quickly. This is close to the situation in which a step force is removed at time zero, as required by the theory. The fluctuation data were collected at approximately the same separation that the tip jumps to after separation from the glue.

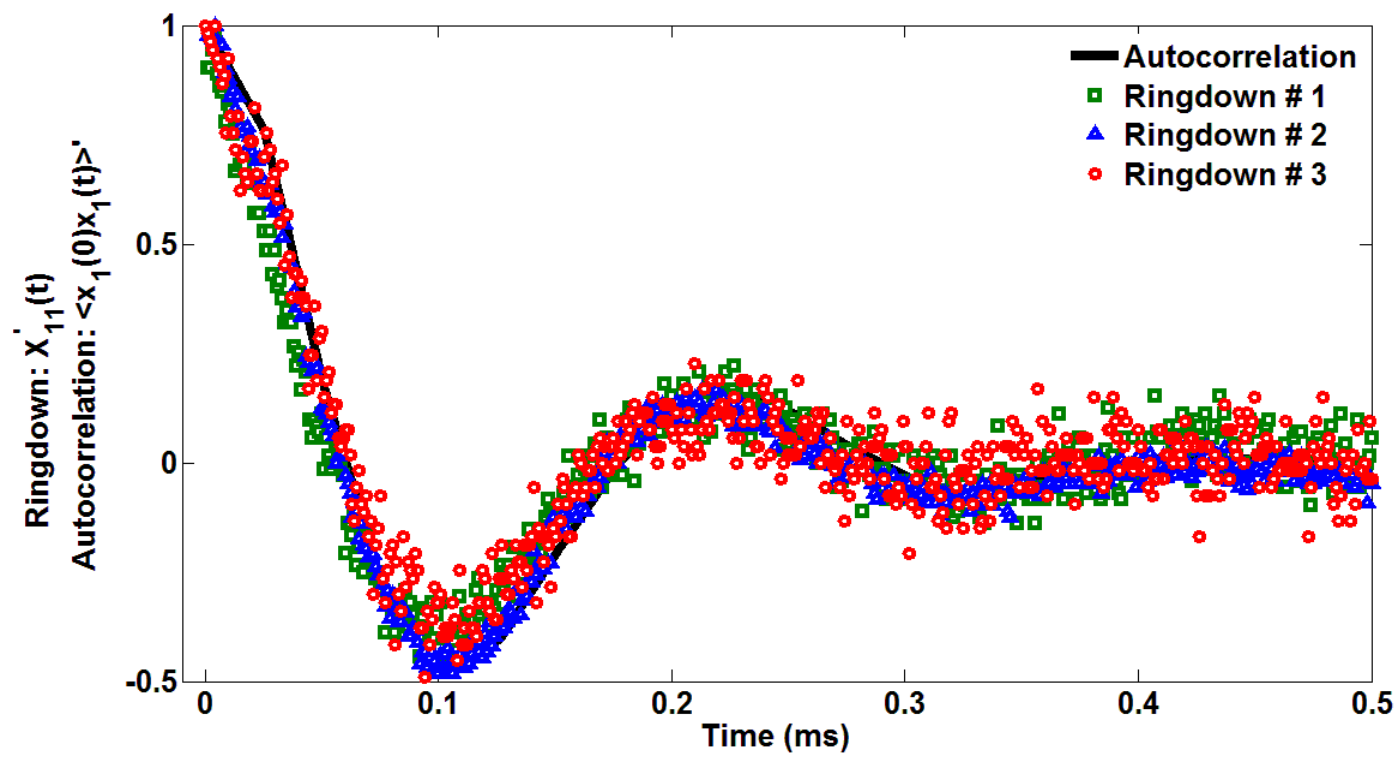

Figure 2-7: Comparison of the left and right sides of equation (2.4). The autocorrelation (normalized by $k_{\mathrm{B}} T / k_{\mathrm{c}}$ ) and the deflection (normalized by its value at time zero) after release of a step force are plotted on the same axis. Both data sets were collected at $1 \mathrm{MHz}$ with ORC8-B type cantilever with spring constant $0.1 \mathrm{~N} / \mathrm{m}$ and resonant frequency $5 \mathrm{kHz}$ in water.

The comparison is shown in Figure 2-7. The similarity between the ringdown and the autocorrelation in thermal fluctuations validates the use of equation (2.4). Since the same 
assumptions were made in the derivation of equation (2.5), it strongly suggests that equation (2.5) is also valid for dual cantilever system. Note that the actual deflection in the ringdown experiment is rather large $(\mathrm{nm})$, much larger than the fluctuations in a normal experiment, so the theory should also be valid for small deflections.

The significance of this result is that the fluctuations of two cantilevers can be measured experimentally, which is an easy measurement, and non-intrusive. The modeling of the system can be performed analytically as the ringdown of cantilevers, which is an "easy" system to model.

\section{Analysis of Thermal Fluctuations to obtain Correlations}

In each experiment, fluctuations of the two cantilevers were measured simultaneously: $x_{1}(t)$ and $x_{2}(t)$ of cantilevers 1 and 2 respectively. In some measurements Asylum Research MFP3D controller was used to collect data at $50 \mathrm{kHz}$ for 50 seconds or National Instrument (Irvine, CA) PCI-6110 Data Acquisition card was used to collect data at $1 \mathrm{MHz}$ for either 5 seconds or 16 seconds. This results in $N$ number of samples for each cantilever. Subsequent data processing consists of:

1. Dividing the whole data points into $N_{\text {ave }}$ bins where each bin has $N_{\text {bin }}=N / N_{\text {ave }}$ number of points

2. Multiplying each bin by a Hanning window ${ }^{3}$ and then subtracting a linear curve fit from each bin to remove drift in the signal (this removes the deflection of the AFM cantilevers that arises from small temperature changes). The theory requires that the system is in a stationary state, i.e. the average deflection of the cantilever is invariant for all bins.

3. Taking a Fourier transform of each bin and calculating the raw power spectral density (PSD) for each cantilever averaged over all bins:

$$
G_{11, \text { raw }}(\omega)=\frac{1}{N_{\text {ave }}} \sum_{\text {bins }} \hat{x}_{1}(\omega) \hat{x}_{1}^{*}(\omega)
$$

\footnotetext{
${ }^{3}$ In signal processing, Hanning window is a window function that helps proper Fourier transforming of time domain fluctuations data to frequency space using FFT (mentioned in data processing step 3) by zeroing the values of data points at the edges of each bin, so as to keep the periodicity of data required by the FFT.
} 


$$
\begin{aligned}
& G_{22, \text { raw }}(\omega)=\frac{1}{N_{\text {ave }}} \sum_{\text {bins }} \hat{x}_{2}(\omega) \hat{x}_{2}^{*}(\omega), \\
& G_{12, \text { raw }}(\omega)=\frac{1}{N_{\text {ave }}} \sum_{\text {bins }} \hat{x}_{1}(\omega) \hat{x}_{2}^{*}(\omega),
\end{aligned}
$$

where $\hat{x}_{i}=F F T\left(x_{i}\right)$, and FFT denotes the fast Fourier Transform which is calculated by Igor Pro software. The asterisk denotes complex conjugate of the Fourier transformed fluctuations. $G_{11 \text {, raw }}$ and $G_{22 \text {, raw }}$ are the (thermal) noise spectra of each cantilever and $G_{12 \text {, raw }}$ is the cross-correlation noise spectrum of the two cantilevers. $\omega$ is frequency in Hz. The unit of $G_{11 \text {, raw }}, G_{22 \text {, raw }}$ and $G_{12 \text {, raw }}$ depends on the units of $x_{1}$ and $x_{2}$, for example if $x_{i}$ is in units of voltage $(V), G_{i j \text {, raw }}$ is in units of $V^{2} / H z$.

So far the data is in instrument-dependent units of volts and need to be converted into deflection distance in meters. There are two methods for conversion from volts to meters.

a.i. From the equipartition theorem, it is known that the area under the first resonant peak in $G_{11}$ or $G_{22}$ is equal to $k_{\mathrm{B}} T / k_{\mathrm{c}}$. Thus we can normalize each of $G_{11, \text { raw }}$ and $G_{22 \text {, raw }}$ by dividing through by their respective areas under the first resonant peak, $A_{i i}$ to obtain $G_{\mathrm{ii}}$. Multiplication of normalized PSDs by $k_{\mathrm{B}} T / k_{\mathrm{c}}$ gives them the appropriate units of a power density spectrum, $\mathrm{m}^{2} / \mathrm{Hz}$. Users of Igor Pro should note that Igor outputs Fourier transforms that are normalized by the Nyquist frequency, $f_{\mathrm{Ny}}$, so in practice, I normalize by $A_{i i} / f_{\mathrm{Ny}}$. The cross-correlation noise spectrum $\left(G_{12, \text { raw }}\right)$ is normalized by the geometric mean of the normalization constant for each contributing signal, i.e. $\sqrt{A_{11} A_{22}} / f_{\mathrm{Ny}}$. The normalized thermal noise spectra are distinguished with a prime.

a.ii. The auto- and cross-correlation functions are then the inverse Fourier transform of the noise spectra for which an Inverse Fast Fourier Transform function is used: $\left\langle x_{i}(t) x_{j}(0)\right\rangle^{\prime}=\operatorname{IFFT}\left(G_{i j}^{\prime}\right)$. Note that the normalization procedure described in step (a.i) gives a value of the auto-correlation of unity at zero time lag: $\left\langle x_{i}(0) x_{i}(0)\right\rangle^{\prime}=1$.

The second method is by using the optical lever sensitivity (OLS) to convert voltage data directly to displacement: 
b.i. First the Inv. OLS $(\mathrm{nm} / \mathrm{V})$ of each cantilever is calculated from the linear region of a force plot (as explained in the previous section). The deflection in volts can thus be converted to meters. The OLS is different for the two cantilevers and depends on the sensitivity of the detection system, laser size and position on the back of the cantilever, and must be measured in each experiment.

b.ii. The thermal noise spectra and cross-correlation noise spectrum are then:

$$
\begin{gathered}
G_{11}=(\text { Inv. OLS })^{2} \frac{G_{11, \text { raw }}}{0.375 \times N_{\text {bin }} f_{\mathrm{Ny}}}, \\
G_{22}=(\text { Inv. OLS })^{2} \frac{G_{22, \text { raw }}}{0.375 \times N_{\text {bin }} f_{\mathrm{Ny}}}, \\
G_{12}=(\text { Inv. OLS } \times \text { Inv. OLS }) \frac{G_{12, \text { raw }}}{0.375 \times N_{\text {bin }} f_{\mathrm{Ny}}},
\end{gathered}
$$

which are in units of $\left(\mathrm{m}^{2} / \mathrm{Hz}\right)$. It is noted the factor $1 / 0.375$ is correction for using Hanning window over each bin.

b.iii. The auto- and cross-correlation functions are then the inverse Fourier transform of the noise spectra for which an Inverse Fast Fourier Transform function is used: $\left\langle x_{i}(t) x_{j}(0)\right\rangle=\operatorname{IFFT}\left(G_{i j}\right)$. It is noted that the procedure described in step (b.ii) gives a value of the auto-correlation of $k_{\mathrm{B}} T / k_{\mathrm{c}}$ at zero time lag: $\left\langle x_{i}(0) x_{i}(0)\right\rangle=k_{\mathrm{B}} T / k_{\mathrm{c}}$.

The first approach is used in the laterally offset CFS where the OLS could not be obtained, and the second method is mainly used with vertically offset system.

It is also possible to perform a modal analysis of the fluctuations data. The modal analysis is realized considering that the two-cantilever system has two orthogonal degrees of freedom. These degrees of freedom are found to be the collective motion of the cantilevers $x_{1}+x_{2}$ and the differential motion of the cantilevers $x_{1}-x_{2}$. The modal analysis is explained in later sections. Calculation of the respective power spectral density of each mode is the same as elaborated above. 


\section{Summary}

The CFS has enhanced force resolution and sensitivity than AFM. This makes CFS a better single molecule force spectroscopy tool. The enhancements are due to decreased damping due to smaller hydrodynamic interaction between two cantilevers and performing cross-correlation measurement. The CFS measurements are described via a deterministic approach using the fluctuation-dissipation theorem. This thesis' objective is now to develop CFS for use in single molecule experiments. For this purpose the vertically offset configuration is the candidate since the thermal fluctuations of the cantilevers exert tensile and compressional tensions on the polymer while in laterally offset configuration the layers of material are sheared between the cantilever ends. However in order to better understand the concept of cross-correlation and how parameters such as fluid density and viscosity, distance between the cantilevers, shape of the cantilever and misalignment of the two levers have influence I start with experiments in the laterally offset configuration which has the benefit of fixed cantilevers in space and minimum thermal drift. In addition, the fact that the fluid layers are sheared between the cantilever ends in laterally offset CFS makes it an interesting case study for applications in microrheology. I will then move on to experiments in vertically offset CFS, which is the target configuration for applications in single molecule force spectroscopy. Initially experiments where no polymer is tethered between the two cantilevers are performed. Then experiments with a polymer tethered between the two cantilevers are performed in order to characterize the polymer. 


\section{Dynamics of Single Molecules}

\section{Elastic Properties of Single Molecules: Worm-Like Chain and Freely- Jointed Chain Models}

The force induced elongation of polymers has been successfully modeled with worm-like chain (WLC) model of elasticity [49]:

$$
F \cong f\left[-2\left(1-\frac{\langle R\rangle}{R_{\max }}\right)+\frac{1}{2}\left(1-\frac{\langle R\rangle}{R_{\max }}\right)^{-2}+\frac{3}{2}\right],
$$

and freely jointed chain (FJC) model of elasticity [49]:

$$
\langle R\rangle=R_{\max }\left[\operatorname{coth}\left(\frac{F}{f}\right)-\frac{f}{F}\right],
$$

where $F$ is the tensile force required to extend the molecule to an average end-to-end distance $\langle R\rangle . f=k_{\mathrm{B}} T / 2 l_{\mathrm{p}}$ is a characteristic force and $l_{\mathrm{p}}$ is the length of persistence segment in WLC model, $k_{\mathrm{B}}$ is the Boltzmann constant, $T$ is the absolute temperature, and $R_{\max }$ is the contour length. It is noted that Kuhn length $b=2 l_{\mathrm{p}}$, and thus $R_{\max }=b N$, where $N$ is the number of Kuhn segments. The FJC model assumes that the chain consists of $N$ segments of Kuhn length $b$ that are freely connected and there is no correlation between the Kuhn segments, while the WLC model assumes a continuous chain where the correlations between a segment on the chain and another segment along the chain becomes significantly small if they are separated by more than one persistence length $l_{\mathrm{p}}$. For $F<f$ the chain has linear entropic elasticity $k=3 k_{\mathrm{B}} T / N b^{2}$. For higher $F$ the entropic elasticity becomes highly non-linear. Equations (3.1) and (3.2) model conformations of ideal chains (see Figure 3-1):

- In the FJC model there is no correlation between bond vectors: $\left\langle\vec{r}_{i} \cdot \vec{r}_{j}\right\rangle=0$ for $i \neq j$ which means freely rotating bond angles $\theta_{i}$. 
- The WLC model assumes that correlation between bond vectors persists but decays exponentially with $l_{\mathrm{p}}:\left\langle\vec{r}_{i} \cdot \vec{r}_{j}\right\rangle=\exp \left(-|i-j| l / l_{p}\right)$, where $l$ is the fixed bond length. The persistence between bond vectors $\vec{r}_{i}$ along a WLC is due to assumption of fixed bond vector angle $\theta_{i}=\theta$.

- Both FJC and WLC models assume freely rotating torsion angles $\varphi_{i}$ and thus ignore energy costs associated with variation in inter-atom distances along the chain (Figure 3-1).

- The WLC model of polymer elasticity has been specifically successful with nucleic acids and polypeptides [50-52].

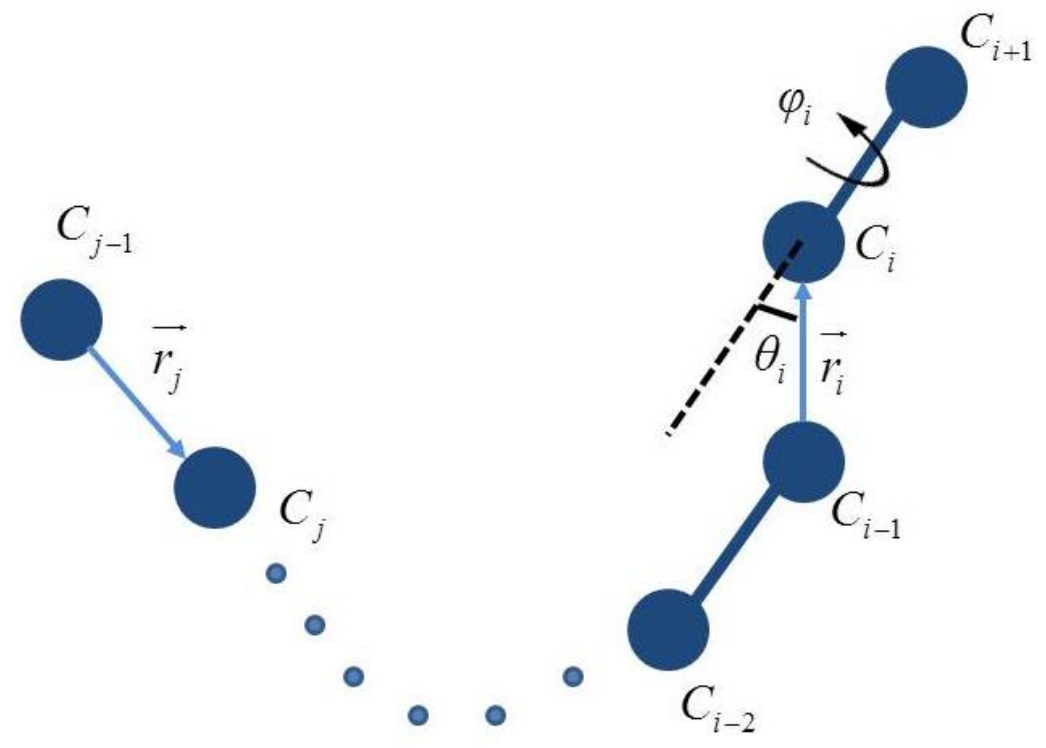

Figure 3-1: Bond angle $\theta_{i}$ between bond vectors $\vec{r}_{i}=\overline{C_{i-1} C_{i}}$ and $\overline{C_{i} C_{i+1}}$ and torsion angle $\varphi_{i}$ of bond vector $\overline{C_{i} C_{i+1}}$ where $\vec{r}_{i}$ is the axis of rotation. There is energy cost associated with variation of torsion angle $\varphi_{i}$ due to variation in distance between atoms $C_{i-2}$ and $C_{i}$. $\left|\overrightarrow{r_{i}}\right|=\left|\vec{r}_{j}\right|=l$ is the bond length.

From equation (3.1), stiffness of a WLC, $k_{\mathrm{wLC}}(=d F / d\langle R\rangle)$, is:

$$
\frac{R_{\mathrm{max}} k_{\mathrm{wLC}}}{f}=H^{-1}\left(\frac{F}{f}\right)
$$


where $H^{-1}$ is the inverse function $H$ :

$$
H(x) \cong-2(x-2)^{-1 / 3}+\frac{1}{2}(x-2)^{2 / 3}+\frac{3}{2} .
$$

The stiffness of a FJC is obtained in a similar way from equation (3.2) that gives:

$$
k_{\mathrm{FJC}}=\frac{f}{R_{\max }} \frac{1}{1-\operatorname{coth}^{2}(F / f)+(F / f)^{-2}} .
$$

Application of equation (3.3), or another equation expressing WLC end-to-end distance probability distribution (see for example Ref. [53]), or equation (3.5) for FJC molecule is to calculate the persistence length $l_{\mathrm{p}}$ or Kuhn length $b$ that are a measure of molecular elasticity.

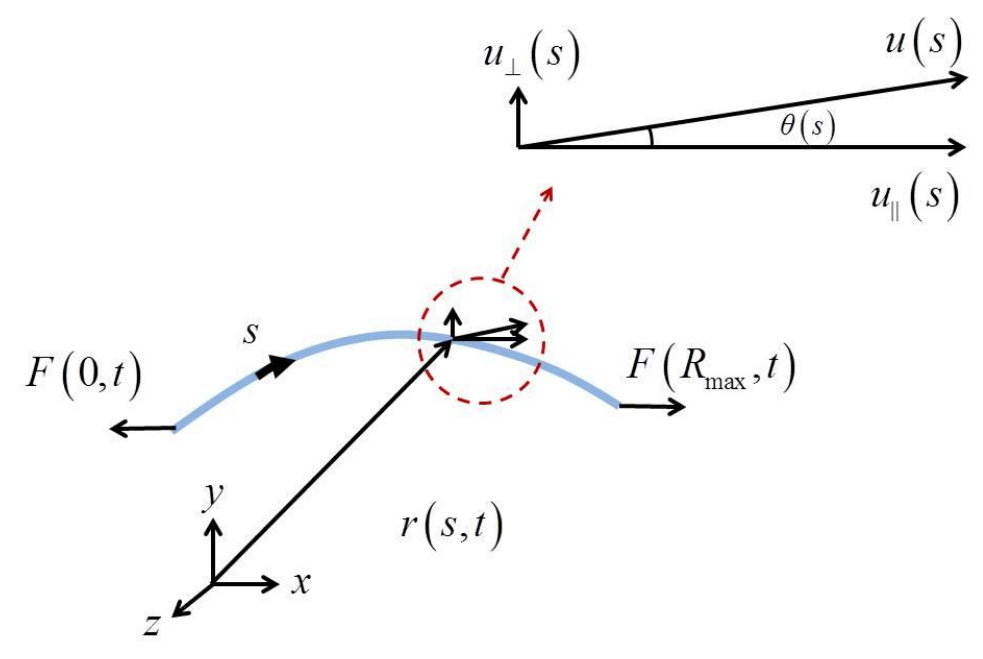

Figure 3-2: Schematic of a WLC continuous space curve.

Due to its relevance to this thesis, here the calculation of WLC elasticity at high force $F \gg f$ is reviewed from Marko and Siggia (1995) [54] and Khatri et al. (2008) [50]. The conformation of a WLC of fixed total length $R_{\max }$ can be described by a conformation space curve $\vec{r}(s, t)$, where $t$ denotes time and $s$ denotes the length along the chain from one end to the other end (see Figure 3-2). The tangent vector to the WLC curve $\hat{u}(s)=\partial \vec{r} / \partial s$ is a unit vector (i.e., $\hat{u} . \hat{u}=1$, the chain is inextensible). The energy of a stretched WLC is: 


$$
E=\int_{0}^{R_{\max }}\left(\frac{\kappa_{\mathrm{B}}}{2}\left(\frac{\partial^{2} r}{\partial s^{2}}\right)^{2}-F \hat{x} \cdot \hat{u}\right) \mathrm{d} s
$$

where $\kappa_{\mathrm{B}}$ is the bending modulus $\left(=l_{\mathrm{p}} k_{\mathrm{B}} T\right.$ in units of $\left.\mathrm{Nm}^{2}\right)$. When large forces are applied, the tangent vector $\hat{u}$ fluctuates only slightly around $\hat{x}$ and thus, for the parallel component of the tangent vector $\left(\vec{u}_{\|}\right)$from Taylor expansion:

$$
\vec{u}_{\|}=\sqrt{1-\vec{u}_{\perp}^{2}} \cong 1-u_{\perp}^{2} / 2,
$$

where $u_{\perp}$ is the perpendicular component of the tangent vector, and $\partial u / \partial s \cong \partial u_{\perp} / \partial s$ since most of the variation is in the perpendicular component $u_{\perp}$ under high tension. Equation (3.6) thus forms:

$$
E=\int_{0}^{R_{\max }}\left(\frac{\kappa_{\mathrm{B}}}{2}\left(\frac{\partial u_{\perp}}{\partial s}\right)^{2}+\frac{F}{2} u_{\perp}^{2}\right) \mathrm{d} s-F R_{\max }
$$

Application of a Fourier transform $\tilde{u}_{\perp}(q) \equiv \int e^{i q s} u_{\perp}(s) d s, u_{\perp}(s)=\sum_{q=-\infty}^{q=+\infty} \tilde{u}_{\perp}(q) e^{i q s}$ and Parseval's theorem $\int u_{\perp}^{2}(s) d s=\sum \tilde{u}_{\perp}^{2}(q)$ gives:

$$
E=\frac{1}{2} \sum\left(\kappa_{\mathrm{B}} q^{2}+F\right) \tilde{u}_{\perp}^{2}(q)
$$

where the constant term is dropped. The partition function of the system is then:

$$
Z=e^{-E / k_{\mathrm{B}} T}=\prod e^{\frac{1}{2}\left(\kappa_{\mathrm{B}} q^{2}+F\right) \tilde{u}_{\perp}^{2}(q) / k_{\mathrm{B}} T} .
$$

The average of $\tilde{u}_{\perp}^{2}$ is thus:

$$
\left\langle\tilde{u}_{\perp}^{2}(q)\right\rangle=\frac{2 k_{\mathrm{B}} T}{\kappa_{\mathrm{B}} q^{2}+F}
$$

The chain extension is:

$$
\langle R\rangle=\int\left(1-\left\langle u_{\perp}^{2}\right\rangle\right) \mathrm{d} s=L-\sum\left\langle\tilde{u}_{\perp}^{2}(q)\right\rangle
$$

which gives:

$$
\langle R\rangle=R_{\max }-\sum \frac{2 k_{\mathrm{B}} T}{\kappa_{\mathrm{B}} q^{2}+F}=R_{\max }\left(1-\frac{k_{\mathrm{B}} T}{2 \sqrt{\kappa_{\mathrm{B}} F}}\right) .
$$


The stiffness of a WLC at high stretch is then $k_{\mathrm{wLC}}=\partial F / \partial\langle R\rangle[50]$ :

$$
k_{\mathrm{WLC}}=\frac{4 \kappa_{\mathrm{B}}^{1 / 2}}{R_{\max } k_{\mathrm{B}} T} F^{3 / 2} .
$$

Thus we have an explicit equation for the stiffness of a polymer chain as a function of the applied force, a parameter that is directly measurable in the CFS and AFM experiments. It is noted that derivation of equation (3.14) is under the assumption of high tension. Equation (3.14) also shows that the molecule gets stiffer with larger bending modulus (or persistence length) and with increasing tension along the chain.

\section{Hydrodynamics of Single Molecules: Dumbbell Model and Rouse Model}

Polymer fluctuations can be modeled in simplest form by dumbbell model. A dumbbell consists of two beads with bead-solvent friction coefficient $\zeta_{\mathrm{s}}$ that are connected by a spring of stiffness $k^{\prime}$ (see Figure 3-3-a). The overdamped dynamics of each bead is:

$$
\zeta_{\mathrm{s}} \frac{d \overrightarrow{R_{1}}}{d t}-k^{\prime}\left(\overrightarrow{R_{2}}-\overrightarrow{R_{1}}\right)=f_{n, 1}(t)
$$

and

$$
\zeta_{\mathrm{s}} \frac{d \overrightarrow{R_{2}}}{d t}-k^{\prime}\left(\overrightarrow{R_{1}}-\overrightarrow{R_{2}}\right)=f_{n, 2}(t)
$$

where $\overrightarrow{R_{1}}$ and $\overrightarrow{R_{2}}$ are the positional vectors of beads 1 and 2 respectively (see Figure $3-3-\mathrm{a}$ ). $f_{n, 1}$ and $f_{n, 2}$ are the Brownian forces on beads 1 and 2 respectively that have two moments:

$$
\left\langle f_{n}(t)\right\rangle=0
$$

and

$$
\left\langle f_{n}(t) f_{n}\left(t^{\prime}\right)\right\rangle=2 k_{\mathrm{B}} T \zeta \delta\left(t-t^{\prime}\right)
$$

where $\delta$ is the Dirac delta function. In this model the polymer has only two degrees of freedom (modes): 


$$
R_{\mathrm{c}}=\frac{\overrightarrow{R_{1}}+\overrightarrow{R_{2}}}{2} \Rightarrow \zeta_{\mathrm{s}} \frac{d R_{\mathrm{c}}}{d t}=\frac{f_{n, 1}(t)+f_{n, 2}(t)}{2}
$$

and

$$
\langle R\rangle=\overrightarrow{R_{1}}-\overrightarrow{R_{2}} \Rightarrow \quad \zeta_{\mathrm{s}} \frac{d\langle R\rangle}{d t}+2 k^{\prime}\langle R\rangle=f_{n, 1}(t)-f_{n, 2}(t),
$$

where $R_{\mathrm{c}}$ is the motion of center of mass vector and $\langle R\rangle$ is the vibration of chain end-to-end vector. $\left(f_{n, 1}+f_{n, 2}\right) / 2$ and $f_{n, 1}-f_{n, 2}$ are the Brownian forces in each mode respectively. The motion of the center of mass can be obtained from diffusion studies. The focus of this thesis is in the motion of the chain end-to-end vector (equation (3.20)). The form of this equation is that of an overdamped simple harmonic oscillator (SHO) in its simplest form where inertial forces due to mass are ignored (see Figure 3-3-b) [55]. For $k=2 k^{\prime}$ and $f_{n}(t)=f_{n, 1}(t)-f_{n, 2}(t)$ equation (3.20) thus becomes:

$$
\zeta \frac{d\langle R\rangle}{d t}+k\langle R\rangle=f_{n}(t)
$$

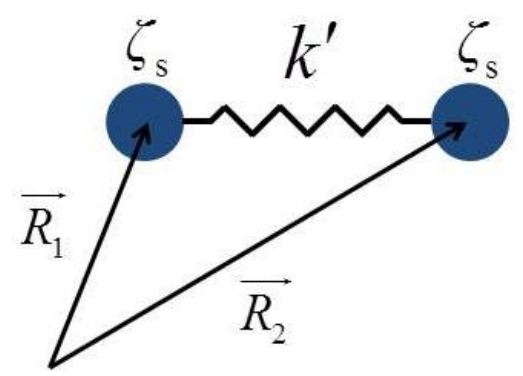

(a)

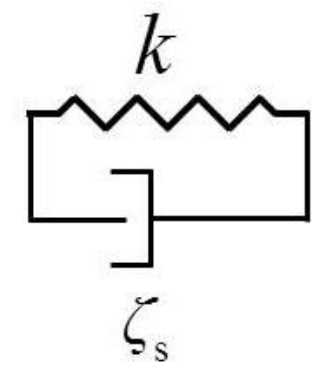

(b)

Figure 3-3: (a) Schematic of a dumbbell. (b) Schematic of a spring with stiffness $k$ and dashpot with friction coefficient $\zeta_{\mathrm{s}}$. This is the model of a highly overdamped simple harmonic oscillator where inetial foces due to mass are ignored.

The compliance of this system (obtained by solving equation (3.21) for $f(t)=\delta(t)$ is:

$$
\chi_{\text {dumbbell }}=\frac{1}{k+i \omega \zeta}=\frac{1}{k} \times \frac{1}{1+i \omega \tau},
$$


where $\omega$ is the frequency and $\tau=\zeta / k$ is the characteristic relaxation time of the chain. The power spectral density (PSD) of a dumbbell can be calculated from [43]:

$$
G_{\text {dumbbell }}=4 k_{\mathrm{B}} T \zeta\left|\chi_{\text {dumbbell }}\right|^{2},
$$

i.e.:

$$
G_{\text {dumbbell }}=\frac{4 k_{\mathrm{B}} T \zeta}{k^{2}} \times \frac{1}{1+\omega^{2} \tau^{2}} .
$$

Equation (3.24) shows that the short time motion of the polymer chain is dominated by its friction: at high frequencies $\omega \gg 1 / \tau, G_{\text {dumbbell }}=4 k_{\mathrm{B}} T / \omega^{2} \zeta^{2}$. For very long times $(\omega \ll 1 / \tau)$ equation (3.24) forms $G_{\text {dumbeell }}=4 k_{\mathrm{B}} T \zeta / k^{2}$. Therefore stiffness dominates the dynamics of the molecule at very long times.
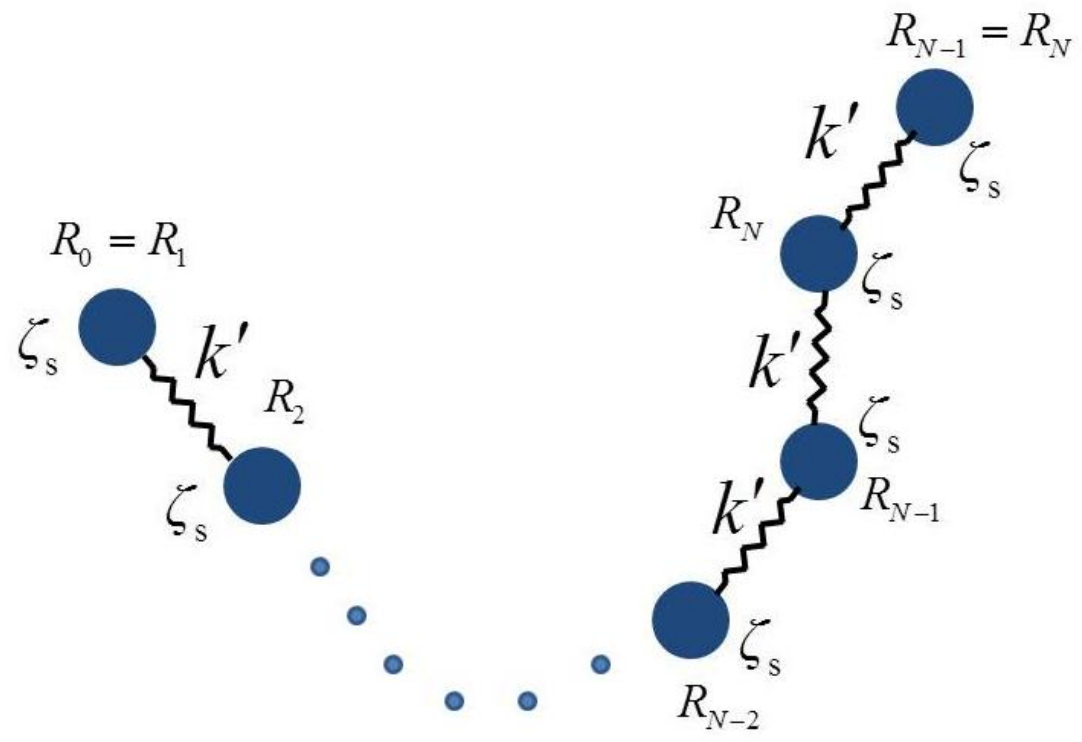

Figure 3-4: Rouse model consists of $N$ beads of bead-solvent friction $\zeta_{\mathrm{s}}$ that are connected by linear springs of stiffness $k^{\prime}$.

The Rouse model consists of $N$ beads that are connected in sequence (see Figure 3-3-a) as shown in Figure 3-4. This connectivity results in many vibration modes along the chain and thus it is a more realistic picture of a polymer chain than the dumbbell model that has only one vibration mode equation (3.20). The modes of the Rouse chain are [56]: 


$$
\overrightarrow{X_{\mathrm{p}}} \equiv \frac{1}{N} \int_{0}^{N} \mathrm{~d} n \cos \left(\frac{p \pi n}{N}\right) \overrightarrow{R_{n}}(t)
$$

where $\overrightarrow{X_{\mathrm{p}}}$ is the mode amplitude and $p=0,1,2, \ldots$ is the mode number. The dynamics of each mode is then similar to dumbbell model equation (3.21) [56]:

$$
\zeta_{\mathrm{p}} \frac{d \overrightarrow{X_{\mathrm{p}}}}{d t}+k_{\mathrm{p}} \overrightarrow{X_{\mathrm{p}}}=f_{\mathrm{p}}(t),
$$

where the mode friction $\zeta_{\mathrm{p}}$ and stiffness $k_{\mathrm{p}}$ are given by [56]:

$$
\zeta_{0}=N \zeta_{\mathrm{s}} \quad \text { and } \quad \zeta_{\mathrm{p}}=2 N \zeta_{\mathrm{s}} \quad \text { for } \quad p=1,2,3, \ldots
$$

and

$$
k_{\mathrm{p}}=\frac{2 \pi^{2} p^{2} k^{\prime}}{N} \text { for } \quad p=0,1,2, \ldots
$$

The relaxation time of each mode is [56]:

$$
\tau_{p}=\frac{\zeta_{p}}{k_{p}}=\frac{N^{2} \zeta_{\mathrm{s}}}{\pi^{2} p^{2} k^{\prime}} \quad \text { for } \quad p=0,1,2, \ldots
$$

The mode $X_{0}\left(=\frac{1}{N} \int_{0}^{N} \mathrm{~d} n \overrightarrow{R_{n}}\right)$ corresponds to the center of mass motion. The vibration modes $X_{\mathrm{p}}$ with $p>0$ represent the internal conformations of the polymer chain with $N / p$ segments. To determine the dynamics of end-to-end vector $\langle R\rangle=\overrightarrow{R_{N}}(t)-\overrightarrow{R_{0}}(t)$, a summation over all modes is required. Khatri [57] performed this summation and showed that the compliance of a Rouse chain is:

$$
\chi_{\mathrm{R}}=\frac{2 N}{\pi k} \frac{\tanh \left(\frac{\pi}{2} \sqrt{i \omega \tau_{\mathrm{R}}}\right)}{\sqrt{i \omega \tau_{\mathrm{R}}}}
$$

where $\tau_{\mathrm{R}}$ is the Rouse relaxation time. As equation (3.30) suggests and is also shown by Dio and Edwards (1998), the dynamics of end-to-end vector $\langle R\rangle$ of a Rouse chain is dominated by the first mode $X_{1}$ that represents the longest relaxation time of the chain:

$$
\tau_{\mathrm{R}}=\tau_{1}=\frac{\zeta_{1}}{k_{1}}=\frac{N^{2} \zeta_{\mathrm{s}}}{\pi^{2} k^{\prime}}
$$


If length of one Kuhn segment $b$ is considered for the bead size, then using Stokes' friction formula:

$$
\zeta_{\mathrm{s}}=6 \pi \eta b,
$$

for $\eta \sim 10^{-3} \mathrm{~kg} / \mathrm{s}$ and $b \sim 10^{-9} \mathrm{~m}$, the friction on one bead is $\zeta_{\mathrm{s}} \cong 0.02 \mu \mathrm{g} / \mathrm{s}$. Using the linear entropic stiffness $3 k_{\mathrm{B}} T / b^{2}$ for the spring connecting the beads $k^{\prime} \cong 0.1 \mathrm{~N} / \mathrm{m}$. Thus for $N=100$ , that is a typical number of Kuhn monomers in single molecule force spectroscopy of short chains, $\tau_{1} \cong 3 \mu \mathrm{s}$. Practically much larger time scales have been found in experimental measurements of single molecules using AFM, and these long time scales have been attributed to dominance of damping between the monomers that is not included in the Rouse model. This damping is often called" Internal friction", a concept that will be discussed in later sections.

The Rouse model, however, is not applicable when the chain is very stiff, for example when the chain is highly stretched or for rigid polymers such as nucleic acids [58]. Under high stretch the chain exhibits non-linear entropic elasticity that is not included in the Rouse model. These nonlinearities are included in elastic models of flexible chains: the worm-like chain model and the freely-jointed chain model.

\section{Internal Friction}

An important outcome of the development of CFS is the ability to accurately measure internal friction. The friction on a polymer chain is the non-conservative force that arises from the velocity of the chain. When the chain moves through the solvent, the friction is usually described as Stokes friction, whereas relative motion of parts of the chain is described as "internal friction", even though most of the heat from this motion is still dissipated via interaction with the solvent. CFS allows us to continuously monitor the end-to-end distance of a polymer chain. As described in Chapter 2, we consider changes in this distance as the sum of two modes: a symmetrical mode and an antisymmetrical mode. The symmetrical mode is movement of both ends in the same direction, the time derivative of which yields the friction due to the entire polymer moving through the solvent. In AFM the friction in the symmetrical mode is obscured by the friction due to the cantilevers also moving through the solution, but that shortcoming is 
not serious as there are other methods for determining the Stokes friction of the polymer. The important feature of CFS is the ability to measure the relative motion of the two ends of the polymer, which corresponds to the internal friction.

The internal friction has been recognized as important during the folding of proteins and DNA $[59,60]$. During folding process, the molecule moves along an energy surface that becomes onedimensional in mechanical SMFS experiments and in the direction of applied force. This energy landscape is not smooth, but is rough due to many intramolecular interactions such as formation and breaking of weak bonds, dihedral angle rotation, interchain collisions or as a result of partial exposure to solvent [61]. The roughness of the energy landscape slows down the folding/unfolding process or other conformational changes, and causes dissipation because the organized motion that causes conformation change is not conserved but lost into the solvent. The relationship between the characteristic time and the damping is given by:

$$
\tau=\frac{\zeta}{k}
$$

Since motion along the energy landscape is due to thermal fluctuations and is thus diffusive, the common theoretical framework for quantification of the energy landscape is Kramers theory [62] and Zwanzing theory [59]. For escape over a smooth potential barrier with height $\Delta G_{0}^{\neq}>k_{B} T$ from the minimum of energy at point A over the transition state st (see Figure 3-5-a), Kramers showed that the rate of crossing the energy barrier $k_{R}$ is:

$$
\frac{1}{k_{R}}=\frac{2 \pi \zeta}{\omega_{0} \omega_{t s}} e^{\Delta G_{0}^{\neq} / k_{\mathrm{B}} T}
$$

It is noted that the potential energies $U$ at $\mathrm{A}$ and at the transition state $s t$ are considered to be harmonic where $\omega_{0}$ describes the curvature of potential $U$ near $\mathrm{A}$ and $\omega_{t s}$ describes the curvature of the potential $U$ at the transition state, and $\zeta$ is the solvent friction coefficient. For diffusion in a rough potential where roughness is randomly distributed in the potential well but has root mean squared amplitude $\varepsilon$ (see Figure 3-5-b), Zwanzig showed that the effective diffusion coefficient is:

$$
D^{*}=D e^{-\left(\varepsilon / k_{\mathrm{B}} T\right)^{2}}
$$


where $D=k_{\mathrm{B}} T / \zeta$ is the diffusion coefficient in a smooth potential background while $D^{*}=k_{\mathrm{B}} T / \zeta^{*}$ is the diffusion coefficient in the rough potential. As a result of this roughness, diffusion is slowed down or in other words $\zeta^{*}>\zeta$.

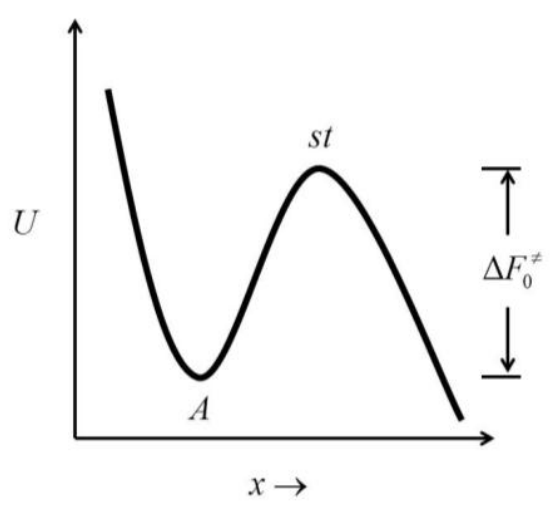

(a)

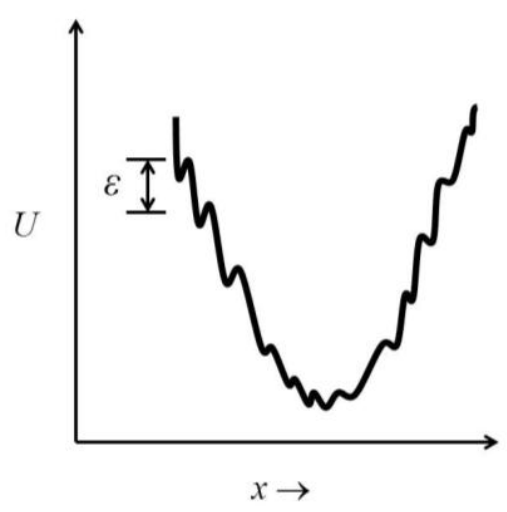

(b)

Figure 3-5: (a) Schematic of the smooth energy landscape $U$ which varies along the reaction coordinate $x . A$ is the minimum energy and $s t$ is the transition state. $\Delta F_{0}^{\neq}$is the activation energy to cross the barrier. (b) Schematic of the rough energy landscape $U$ with root mean squared roughness $\varepsilon$.

In ensemble measurements, the internal friction of polymer chains is investigated by studying folding or binding kinetics at varying solvent viscosity with attempt to elucidate the molecular origin of internal friction. Wensley et al. [63] produced chevron plots of protein folding kinetics to provide an indirect evidence for the roughness of energy landscape in folding and unfolding pathways of the $15^{\text {th }}, 16^{\text {th }}$, and $17^{\text {th }}$ domains of $\alpha$-spectrin (referred to as R15, R16 and R17). They show that despite similarities in structure and stability of the transition states of these proteins, R15 exhibits much faster (about 3000 times faster) folding kinetics than R16 and R17. By varying the viscosity of the solvent (by using glucose) while keeping a constant structural stability (by using denaturant guanidinium chloride) they showed that the folding and unfolding kinetics of R16 and R17 does not follow equation (3.34) if only the viscosity of solvent is considered in the friction term $\zeta$. Adding an internal friction term (in units of viscosity) to the solvent viscosity, they show that the internal friction term is one order of magnitude larger than solvent viscosity, and thus the roughness of the energy landscape is about $\varepsilon \sim 2 k_{\mathrm{B}} T$ from 
equation (3.35). Soranno et al. [61] studied the effect of internal friction on reconfiguration times of unfolded and intrinsically disordered proteins using fluorescence resonance energy transfer (FRET), nanosecond fluorescence correlation spectroscopy, and microfluidic mixing. Using the coarse-grained theoretical framework of Rouse with internal friction model (see Section Rouse with Internal Friction) they studied the effect of solvent viscosity, segment length and sequence composition on the internal friction of proteins. Their results suggest that the origin of internal friction is interaction between amino acid residues that are only partially exposed to solvent. Liu et al. [64] used laser temperature-jump relaxation experiment to study the effect of temperature on roughness of energy landscape during folding of protein FiP35. They found that the roughness increased by temperature that is attributed to the increased hydrophobic effect (being the initial cause of roughness) leading to stronger contacts of the polypeptide chain. They showed that the roughness increased by $\Delta \varepsilon \cong 0.5 k_{\mathrm{B}} T$ for temperature varying 60 to $83^{\circ} \mathrm{C}$. Based on the theory developed by Hyeon and Thirumalai [60] that combines equations (3.34) and (3.35) for two state conformational transition on a rough potential landscape:

$$
\log \left(\frac{k_{R}^{-1}}{k_{0}^{-1}}\right)=\frac{\Delta G^{\neq}-F \Delta x}{k_{\mathrm{B}} T}+\left(\frac{\varepsilon}{k_{\mathrm{B}} T}\right)^{2},
$$

where $k_{0}^{-1}=2 \pi \zeta / \omega_{0} \omega_{t s}, \Delta x$ is the length of transition state $s t$ from point A (see Figure 3-5), and $F$ is force, Schlierf and Rief [65] measured a roughness of $\sim 4 k_{\mathrm{B}} T$ for dictyostelium discoideum filamin (ddFLN4) using conventional AFM pulling experiments.

Internal friction also has a significant effect on DNA dynamics and DNA binding to proteins. For example high internal friction as well as stiffness results in higher stability of DNA-protein complex that can resist either folding or changing conformation [61, 66]. For dextran it was shown that the internal friction increases with decreasing number of Kuhn segments and increasing tensile force [67] while molecule-solvent friction is proportional to the number of Kuhn segments and is not a function of force. Recently it was shown that internal friction varies substantially along the folding pathway of a peptide chain which suggests a connection between friction and formation of hydrogen bonds upon folding [66]. While such studies on proteins are 
ubiquitous [68-70], there is obviously a lack of experimental data on roughness of DNA and RNA conformational transition.

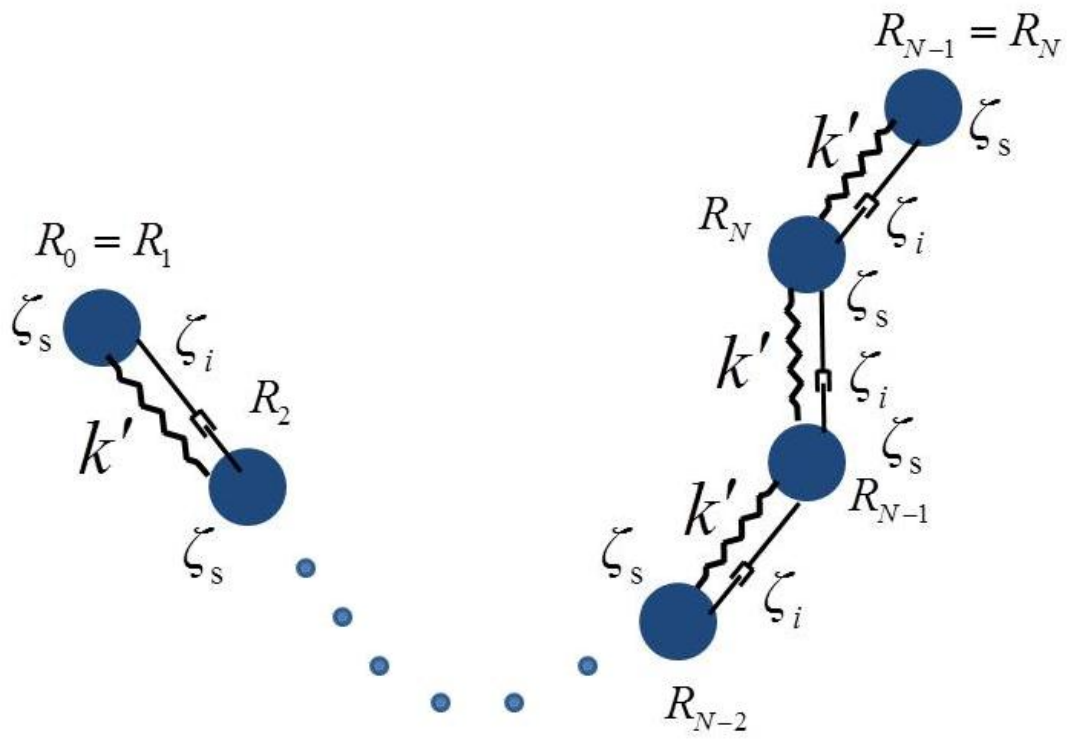

Figure 3-6: Schematic of RIF model. The beads are connected with springs of stiffness $k^{\prime}$ and dashpots of friction $\zeta_{i}$ (internal friction).

\section{Rouse with Internal Friction}

A coarse-grained model of internal friction in polymers was derived based on Rouse model and is called Rouse with internal friction (RIF) [67]. A schematic of the model is shown in Figure 3-6. The internal friction is a phenomenological parameter of polymer dynamics that is due to dissipation during conformational changes (e.g. a change in torsion angle (see Figure 3-1) or during rupture of intramolecular bonds. For example, Khatri et al attributed internal friction in dextran to conformational transitions in the pyranose ring in dextran [67]. Derivation of governing equations of RIF can be found in Khatri and Mcleish (2007) and is not repeated here. The resulting stiffness is similar to equation (3.28) and the RIF friction is [67]:

$$
\zeta_{0}=N \zeta_{\mathrm{s}} \quad \text { and } \quad \zeta_{\mathrm{p}}=2 N \zeta_{\mathrm{s}}+\frac{2 \pi^{2} p^{2} \zeta_{i}}{N} \text { for } p=1,2,3, \ldots
$$

Two immediate conclusions out of equation (3.37) are that internal friction scales inversely with the number of monomers $N$ whereas the molecule-solvent friction that scales linearly with $N$, and 
that the internal friction does not have any effect in the center of mass motion. The compliance of RIF is [57]:

$$
\chi_{\mathrm{RIF}}=\frac{N}{\pi k^{\prime}} \frac{\tanh \left(\frac{\pi}{2} \sqrt{\frac{i \omega \tau_{\mathrm{R}}}{1+i \omega \tau_{\mathrm{i}}}}\right)}{\sqrt{i \omega \tau_{\mathrm{R}}\left(1+i \omega \tau_{\mathrm{i}}\right)}},
$$

where $\tau_{\mathrm{R}}$ is the Rouse relaxation time equation (3.31) and $\tau_{\mathrm{i}}$ is the relaxation time of internal friction:

$$
\tau_{\mathrm{i}}=\frac{\zeta_{\mathrm{i}}}{k^{\prime}}
$$

For a biomolecule with dominant internal friction (e.g. dextran and cellulose [71] and polypeptides [50]) $\tau_{\mathrm{i}} \gg \tau_{\mathrm{R}}$ and thus equation (3.38) simplifies to:

$$
\chi_{\mathrm{RIF}}=\frac{N}{k^{\prime}} \times \frac{1}{1+i \omega \tau_{\mathrm{i}}}
$$

that resembles the response of a dumbbell model equation (3.22) with $N$ atoms in a chain, each connected by a spring and dashpot. This conclusion is very important that it shows that the response of a molecule with dominant internal friction is that of a single mode dumbbell chain.

\section{Model of Linear Viscoelasticity of a Semiflexible Chain}

As mentioned earlier, the Rouse model or Rouse with internal friction are not good models for stiff chains [58]. In an AFM or CFS experiment, it is usual to stretch the polymer somewhat feel a force and therefore to know that the polymer is influencing the cantilever. Thus, almost all AFM-based experiments (including the work here) investigate highly-stretched polymers, and we require theories that incorporate the rigidity of chains.

Here I discuss work by Hiraiwa and Ohta (2010) [58] that incorporates internal friction into the WLC model. The dynamics of a worm-like chain (shown in Figure 3-2) is governed by Langevin equation (overdamped, not included inertia):

$$
\left(\zeta_{\mathrm{s}}+\zeta_{\mathrm{B}} \frac{\partial^{4}}{\partial s^{4}}\right) \frac{\partial r(s, t)}{\partial t}=-\kappa_{\mathrm{B}} \frac{\partial^{4} r(s, t)}{\partial s^{4}}+\frac{\partial}{\partial s}\left(\ell(s, t) \frac{\partial r(s, t)}{\partial t}\right)+f_{n}(s, t) .
$$


where the coefficients $\zeta$ is the friction coefficient due to polymer-solvent friction (in units of viscosity $\mathrm{N} \mathrm{s} \mathrm{m}^{-2}$ ) and $\zeta_{\mathrm{B}}$ is the bending internal friction due to conformational change (in units of $\left.\mathrm{N} \mathrm{s} \mathrm{m}^{2}\right) . \quad \ell(s, t)$ is the line tension and $f_{n}(s, t)$ is the random force. Equation (3.41) was solved by Poirier and Marko (2002) [72] without consideration of polymer-solvent friction term and by Khatri et al. (2008) [50] for extremely long experimental times (the low frequency limit) and high line tension. Although our CFS operates at high frequency $(\mathrm{kHz})$ compared to conventional AFM pulling experiments $(\mathrm{Hz})$ it is still in the low frequency limit. The characteristic time for low frequency limit solution to equation (3.41) is defined by:

$$
\tau_{\mathrm{fs}}=R_{\max }^{2} F^{-1} \zeta_{\mathrm{s}, \perp}\left(1+\frac{\zeta_{\mathrm{B}, \perp}}{\zeta_{\mathrm{s}, \perp}\left(\kappa_{4} / F\right)^{2}}\left(\pi / R_{\max }\right)^{4}\right),
$$

where $\zeta_{\mathrm{s}, \perp}$ is the transverse component of polymer-solvent friction, and $\zeta_{\mathrm{B}, \perp}$ is the transverse component of polymer internal friction. For $\omega \tau_{\mathrm{fs}} \ll 1$ (this is the case in CFS experiments shown in this thesis) the line tension $\ell(s, t)$ is assumed to be uniform along the chain:

$$
\ell(s, t)=\ell(t)=F+\ell_{\mathrm{A}} \sin (\omega t),
$$

and the dynamics of the chain is that of a dumbbell model introduced earlier. In the limit of large external force $F$ :

$$
\frac{F R_{\max }^{2}}{\kappa_{4} \pi^{2}} \gg 1
$$

and

$$
\frac{\pi^{2}\left(F / \kappa_{4}\right)^{3 / 2} \zeta_{\mathrm{B}, \perp}}{8 \zeta_{\mathrm{s}, \perp} R_{\max }} \gg 1
$$

the relaxation time of the chain is:

$$
\tau=\frac{\zeta_{\mathrm{B}, \perp}}{8 \kappa_{4}}
$$

which indicates that the relaxation time is independent of force $F$. This conclusion is tested by experiment in this thesis. The treatment by Khatri et al. [50] also results in a force independent relaxation time that is a characteristic of dumbbell model. They calculate the dissipation of energy along the chain due to bending via Rayleigh dissipation function [50]: 


$$
D_{\mathrm{wLC}}=\frac{\zeta_{\mathrm{B}}}{2} \int_{0}^{R_{\max }} \mathrm{d} s\left(\frac{\partial \dot{r}}{\partial s^{2}}\right)^{2}=\frac{\zeta_{\mathrm{B}}}{2} \int_{0}^{R_{\max }} \mathrm{d} s\left(\frac{\partial \dot{\theta}}{\partial s}\right)^{2},
$$

where the second equality is for high stretching force $F$ (see Figure 3-2), where the tangent vector $u(s)=\partial r / \partial s$ points almost along the force in $x$ direction. This suggests that $\partial u / \partial s \cong \partial u_{\perp} / \partial s$. This also means that $u_{\perp}(s) \cong \theta(s)$. Thus $\partial^{2} r / \partial s^{2} \cong \partial \theta / \partial s$. For friction of a worm-like chain Khatri et al. find:

$$
\zeta_{\mathrm{wLC}}=\frac{\zeta_{\mathrm{B}}}{8 \kappa_{\mathrm{B}}^{1 / 2} L k_{\mathrm{B}} T} F^{3 / 2},
$$

which predicts that the friction increases as a power law $F^{3 / 2}$. This prediction is tested in Chapter 7 of this thesis. The relaxation time is similar to equation (3.46) but with a different prefactor:

$$
\tau_{\mathrm{wLC}}=\frac{\zeta_{\mathrm{wLC}}}{k_{\mathrm{wLC}}}=\frac{\zeta_{\mathrm{B}}}{32 \kappa_{\mathrm{B}}},
$$

where $k_{\mathrm{wLC}}$ is from equation (3.14). Again the relaxation time is predicted to be independent of the applied force.

\section{Summary}

Internal friction is now known to be important in determining the dynamics of polymers, for example in determining the rate of protein folding. Theoretical predictions using simple polymer models suggest that internal friction should scale with a $3 / 2$ power law of the applied force, and that the characteristic time should be independent of the applied force. CFS measurements are amenable to extraction of the friction due to non-center of mass motions, and therefore can be used to measure internal friction and test these predictions. This document will test these predictions in Chapter 7. 


\section{Microrheology with Correlation Force Spectroscopy}

Rheological applications are expected for both laterally offset CFS and vertically offset CFS because the cross-correlation depends on the viscous coupling between the AFM tips. Understanding the solvent contribution to this coupling is an important precursor to single molecule measurements since the most interesting single molecule studies are performed in solution.

\section{Existing Techniques of Rheometry}

Although the conventional techniques of rheology, stress-controlled or strain-controlled mechanical shear-rheometer, are extremely versatile, they suffer from a number of limitations: the instruments require several milliliters of sample, they have limited range of oscillation frequencies, and the spacing between the opposing plates is of order one centimeter, as a result, the measured response obtained is an average of the bulk response that does not provide information about the local dynamics of heterogeneous systems [73]. Recently, microrheology techniques were developed that overcome many of the limitations [74-79]. The most common of these are the one-particle [80, 81], and two-particle micro-rheometers [77]. Although microrheology techniques are very successful they have limits of applicability that suggest the need for complementary techniques. The video tracking technique used to measure particle displacements is limited to frequencies less than $30 \mathrm{~Hz}$ (half the video frequency) [82]. Obtaining higher frequency response information has only been achieved using diffusing wave spectroscopy (DWS), which requires a concentrated particle suspension, or laser trapping of the two probe particles in conjunction with fast photodiodes [75, 83, 84]. This can be an advantage for materials that have a broad-range of characteristic time scales. The video tracking techniques typically can only resolve displacements of $\sim 20 \mathrm{~nm}$ [85]. The particle-particle separation distance in two-point microrheology must typically be of order one micron [8]. The video techniques also require that particles are large enough to be visualized $(>0.5 \mu \mathrm{m})$. These 
techniques are most effective for 'soft' systems with elastic moduli $<100 \mathrm{~Pa}$ [82]. There are parallel efforts in microrheology measurements using microcantilevers [45, 86-88], following on recent success in understanding the dynamics of cantilevers in fluid [89-98]. A wide range of frequencies and viscous and elastic moduli can be explored by AFM microcantilevers. However, application of two cantilevers in proximity of each other makes possible probing heterogeneous systems. The principal disadvantage of CFS (in common with AFM and particle rheology) for rheology is that the shear stress at each frequency is not constant through the fluid. Exact solutions to the Navier-Stokes equation (for AFM) or unsteady Stokes equation (for one-point and two-point microrheology) can result in a precise knowledge of the dispersion of shear stresses through the fluid at each oscillation frequency of the micro-probe, but this is analytically cumbersome compared to traditional rheometry. Solutions are sometimes sought using finite element analysis [43, 89, 93, 96, 99].

\section{Experimental Methods}

Experiments were performed as explained in Chapter 2. Three Newtonian fluids were used: water $(\eta=0.94 \mathrm{mPa} . \mathrm{s})$, n-pentane $(\eta=0.22 \mathrm{mPa} . \mathrm{s})$ and $\% 24$ glycerol solution $(\eta=2.07 \mathrm{mPa} . \mathrm{s})$. ORC-8 B cantilevers were used and the lateral separation between the levers was set to $8 \mu \mathrm{m}$.

\section{Comparison to Finite Element Analysis}

Finite-element numerical simulations of the deterministic motion of cantilevers, after removal of a step force, for the precise geometries and conditions of the experiment were also done [46, 100]. For simulations, nominal values of the cantilever geometry provided by the manufacturer were used to fit the values for the density $\left(4166 \mathrm{~kg} / \mathrm{m}^{3}\right)$ and Young's modulus $(140 \mathrm{GPa})$ of the cantilever to ensure that the theoretical values of the resonant frequency in air and spring constant of each cantilever matched the corresponding experimentally-measured value. This was done because of the large experimental errors in determining precise values of the Young's modulus and the cantilever thickness. To compute the deterministic dynamics Dr. Mark Paul and Mr. Brian Robbins have numerically solved the time-dependent three-dimensional equations that govern the underlying fluid-solid interaction problem for two cantilevers using the finite 
element based ACE+ Solver. They removed a step force to cantilever 1 and then simulated the deterministic rings of each cantilever. The ringdown of cantilever 1 was used to determine the autocorrelation of cantilever 1 (equation (2.4)) and the rings of cantilever 2 was used to determine the cross-correlation (equation (2.5)). A comparison between the measured autocorrelation and cross-correlation for two cantilevers and the values determined from equations (2.4) and (2.5) using finite element numerical simulations of deterministic motion are shown in Figure 4-1 and Figure 4-2 respectively. The close fit between the experiment and the model using the theory was obtained using the known values of the viscosity and density. This suggests that the analysis of the correlated vibrations of two cantilevers can be used as a rheometer to measure the unknown viscosities of solutions. It is noted that in the simulations the end of cantilevers is $10 \mu \mathrm{m}$ long and there is a single step on either side that is $4.5 \mu \mathrm{m}$ high and $7.5 \mu \mathrm{m}$ long to approximate the $59^{\circ}$ taper on the real cantilever. The prime on the left axis indicates that the correlation function is normalized.
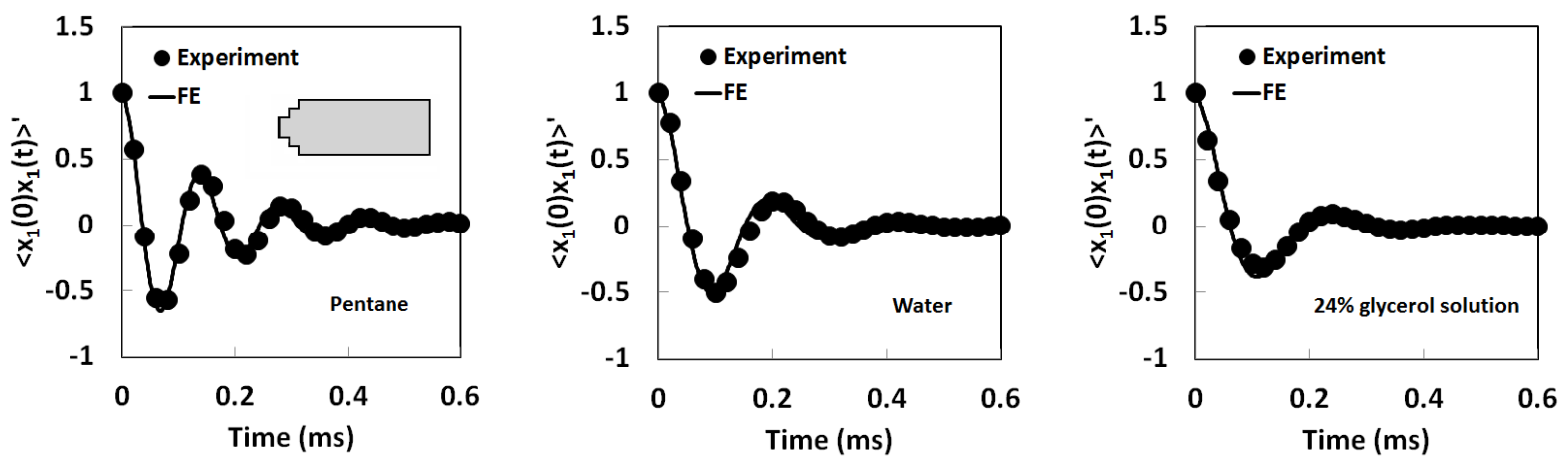

Figure 4-1: Autocorrelation of equilibrium fluctuations in cantilever displacement in a series of Newtonian fluids. Experimental measurements at $23^{\circ} \mathrm{C}$ are shown as data symbols and theoretical predictions are shown by the solid lines. Commercial AFM cantilevers are used for the experiments (ORC8 B: length $=200 \mu \mathrm{m}$, width $=40 \mu \mathrm{m}, \mathrm{k}=0.1 \mathrm{Nm}^{-1}$ ). The simulations used a cantilever with a stepped end as shown in the inset. The prime on the left axis indicates normalized correlation function. 

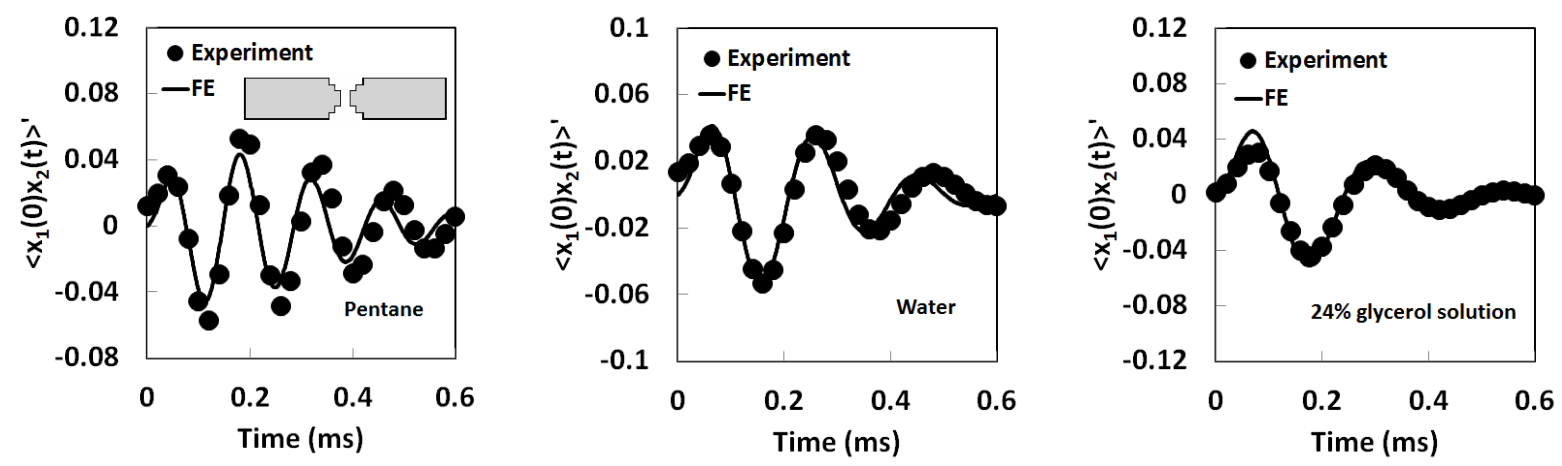

Figure 4-2: Cross-correlation of equilibrium fluctuations in cantilever displacement for a pair of AFM cantilevers in a series of Newtonian fluids. Experimental measurements are shown as data symbols and theoretical predictions are shown by the solid lines. Commercial AFM cantilevers are used (ORC8 B: length $=200 \mu \mathrm{m}$, width $=40 \mu \mathrm{m}, \mathrm{k}=0.1 \mathrm{Nm}^{-1}$ ). Experiments were performed at $23^{\circ} \mathrm{C}$. The cantilevers are separated by $8 \mu \mathrm{m}$. The prime on the left axis indicates normalized correlation function.

\section{Comparison to Simple Harmonic Oscillator Model}

I also tested whether the two cantilever dynamics could be modeled using a pair of coupled simple harmonic oscillators. It is well known that a single cantilever in fluid can be represented as a damped simple harmonic oscillator, where the fluid loaded mass of the cantilever is shown as a lumped mass, $m$, the damping on the cantilever is shown by $\gamma_{a}$, and the spring constant of the cantilever is $k_{\mathrm{c}}$ (see Figure 4-3). The internal damping of the cantilever has been neglected since it is much smaller than the viscous contributions. Both $m$ and $\gamma_{\mathrm{a}}$ are frequency dependent parameters [43]. The fluid loaded mass is different from and higher than the mass of the cantilever itself since the cantilever carries a column of fluid as it fluctuates in space. When the viscous dissipation is significant (as in a highly viscous fluid when the quality factor, $Q$, is low), the frequency dependence of the fluid loaded mass and damping terms must be included, but in the limit of small dissipation from the fluid, the fluid loaded mass and the viscous damping can be considered frequency independent, and one can evaluate the properties of the fluid at the resonant frequency of the cantilever in the fluid with a small amount of error. 


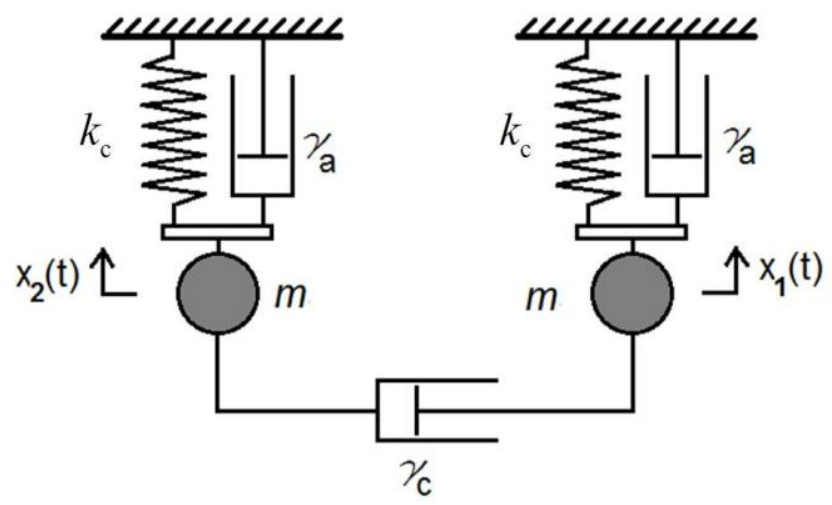

Figure 4-3: Schematic of a cantilever pair modeled as a mass-spring-damper system. $m$ is the effective (i.e. averaged over all frequencies) fluid loaded mass of the cantilever, $k_{\mathrm{c}}$ is the spring constant, and $\gamma_{\mathrm{a}}$ is the coefficient of fluid damping on an individual cantilever. $\gamma_{\mathrm{c}}$ is the coefficient of fluid damping due to the fluid spanning the gap between the two cantilevers. The dashpot for $\gamma_{c}$ works such that when the left mass moves up the right mass experiences a force in the downward direction, and vice versa. The plates shown in the schematic are massless.

The idea of simple harmonic oscillator modeling can be extended to a cantilever pair in fluid. Here the cantilevers are assumed to be identical. The coefficient of hydrodynamic damping of the fluid spanning the gap between the two cantilevers is $\gamma_{\mathrm{c}}$. Similar to $m$ and $\gamma_{\mathrm{a}}$ it is expected that $\gamma_{\mathrm{c}}$ is a function of frequency but in the harmonic oscillator model a single numerical value is obtained by fitting to experimental data.

The equations of motion for the system shown in Figure 4-3 are:

$$
\begin{aligned}
& \ddot{X}_{1}+\left(\frac{\gamma_{\mathrm{a}}}{m}+\frac{\gamma_{\mathrm{c}}}{m}\right) \dot{X}_{1}+\frac{\gamma_{\mathrm{c}}}{m} \dot{X}_{2}+\omega_{r}^{2} X_{1}=0, \\
& \ddot{X}_{2}+\left(\frac{\gamma_{\mathrm{a}}}{m}+\frac{\gamma_{\mathrm{c}}}{m}\right) \dot{X}_{2}+\frac{\gamma_{\mathrm{c}}}{m} \dot{X}_{1}+\omega_{r}^{2} X_{2}=0 .
\end{aligned}
$$

For the initial conditions $X_{1}(0)=F_{1} / k_{c}, \quad X_{2}(0)=0$, and $\dot{X}_{1}(0)=\dot{X}_{2}(0)=0$, the solution to equations (4.1) and (4.2) are: 


$$
\begin{aligned}
& X_{1}=\frac{\exp \left(-\omega_{\mathrm{d}} t\right)}{2}\left[\cos \left(\sqrt{\omega_{\mathrm{r}}^{2}-\omega_{\mathrm{d}}^{2}} t\right)+\frac{\omega_{\mathrm{d}}}{\sqrt{\omega_{\mathrm{r}}^{2}-\omega_{\mathrm{d}}^{2}}} \sin \left(\sqrt{\omega_{\mathrm{r}}^{2}-\omega_{\mathrm{d}}^{2}} t\right)\right] \\
& +\frac{\exp \left(-\frac{\gamma_{\mathrm{a}}}{2 m} t\right)}{2}\left[\cos \left(\sqrt{\omega_{\mathrm{r}}^{2}-\left(\frac{\gamma_{\mathrm{a}}}{2 m}\right)^{2}} t\right)+\frac{\frac{\gamma_{\mathrm{a}}}{2 m}}{\sqrt{\omega_{\mathrm{r}}^{2}-\left(\frac{\gamma_{\mathrm{a}}}{2 m}\right)^{2}}} \sin \left(\sqrt{\omega_{\mathrm{r}}^{2}-\left(\frac{\gamma_{\mathrm{a}}}{2 m}\right)^{2}} t\right],\right. \\
& X_{2}=\frac{\exp \left(-\omega_{\mathrm{d}} t\right)}{2}\left[\cos \left(\sqrt{\omega_{\mathrm{r}}^{2}-\omega_{\mathrm{d}}^{2}} t\right)+\frac{\omega_{\mathrm{d}}}{\sqrt{\omega_{\mathrm{r}}^{2}-\omega_{\mathrm{d}}^{2}}} \sin \left(\sqrt{\omega_{\mathrm{r}}^{2}-\omega_{\mathrm{d}}^{2}} t\right)\right] \\
& -\frac{\exp \left(-\frac{\gamma_{\mathrm{a}}}{2 m} t\right)}{2}\left[\cos \left(\sqrt{\omega_{\mathrm{r}}^{2}-\left(\frac{\gamma_{\mathrm{a}}}{2 m}\right)^{2}} t\right)+\frac{\frac{\gamma_{\mathrm{a}}}{2 m}}{\sqrt{\omega_{\mathrm{r}}^{2}-\left(\frac{\gamma_{\mathrm{a}}}{2 m}\right)^{2}}} \sin \left(\sqrt{\omega_{\mathrm{r}}^{2}-\left(\frac{\gamma_{\mathrm{a}}}{2 m}\right)^{2}} t\right],\right.
\end{aligned}
$$

where $\omega_{\mathrm{r}}=\sqrt{k_{\mathrm{c}} / m}$ is the resonance frequency $(\mathrm{rad} / \mathrm{s})$ and $\omega_{\mathrm{d}}=\left(\gamma_{\mathrm{a}} / 2 m+\gamma_{\mathrm{c}} / m\right)$. The initial displacement of cantilever $1, F_{1} / k_{\mathrm{c}}$, scales all displacements so that equations (4.3) and (4.4) are normalized by $F_{1} / k_{\mathrm{c}}$. Derivation of equations (4.3) and (4.4) is provided in Appendix I: Simple Harmonic Oscillator Model for Laterally Offset Correlation Force Spectroscopy.

Parameters in the equations of motion (4.3) and (4.4) are obtained as follows: the stiffness of each cantilever was measured in advance by thermal method, which is an analysis of the frequency spectrum of the autocorrelation of the isolated cantilever in the immersion fluid. In principle, $\gamma_{\text {a }}$ could be obtained from the frequency spectrum of the isolated cantilever but in practice, the parameters $\omega_{r}, \gamma_{\mathrm{a}}$ and $\gamma_{\mathrm{c}}$ were fitted to obtain the least sum of the normalized errors of auto- and cross-correlation between the experiment with two cantilevers and equations (4.3) and (4.4). The lumped fluid loaded mass for each cantilever was obtained from $m=k_{\mathrm{c}} /\left(2 \pi \omega_{r}\right)^{2}$. Because frequency dependence of $\gamma_{\mathrm{a}}, \gamma_{\mathrm{c}}$ and $m$ is neglected it is expected that the HO model will give better fits for fluids with a narrower resonant frequency, i.e. higher $Q$ factor. 
Figure 4-4 shows the measured water and pentane data along with the fit using the HO model and the fluctuation-dissipation theorem; the $\mathrm{HO}$ fit is good, it clearly captures the observed behavior. The fit parameters are shown in Table 4-1. Comparing water to pentane it is obvious that values of hydrodynamic damping coefficients as well as the fluid loaded mass are higher for a more viscous and denser fluid, so the model captures the essential features of the fluid. The fit in water (Figure 4-4-c and d) is good but not as good as for pentane (Figure 4-4-a and b). This is expected because the assumption of frequency independent fluid loaded mass and damping is worse for more viscous fluids.

From the fit parameters other parameters such as the fluid loaded mass, $m$, and quality factor, $Q$, can be calculated. $\quad \gamma_{\mathrm{a}, \mathrm{th}}$ is calculated from equation (29) in [43] for an infinite cylinder oscillating in fluid, whereas $\gamma_{\mathrm{a}}$ is obtained for the actual geometry of the cantilever in the fluid including tip effects and interaction with the second cantilever. Nevertheless, the theoretical estimate is similar to the experimental value for pentane but about $50 \%$ larger for water.

The HO fit and FE simulation are compared in Figure 4-4-a and b. Recall that the FE simulation uses only fitted values of modulus and density obtained from the power spectrum in air, so it is essentially predictive once the apparatus (the cantilever) has been calibrated. In contrast, the HO model is fitted directly to the data in each fluid. The advantage of the HO model is that it can be obtained very quickly $(<1 \mathrm{~s})$ compared to the FE simulation ( week using a single workstation) and can be used to obtain intuitively useful modeling parameters such as $\gamma_{c}$. It can be easily modified to include a spring and damper between the cantilevers to represent a single molecule. 


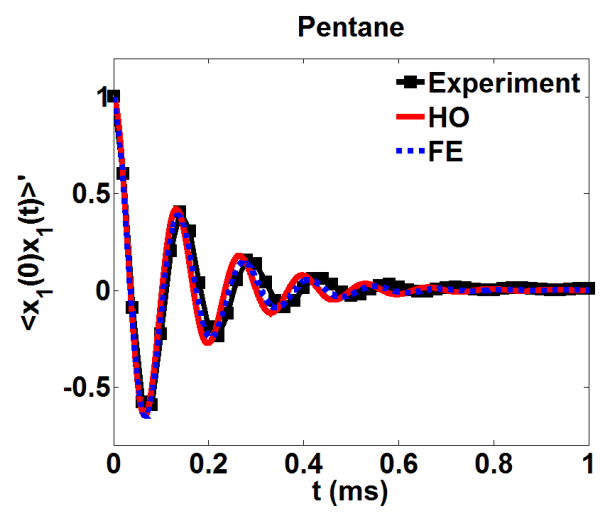

(a)

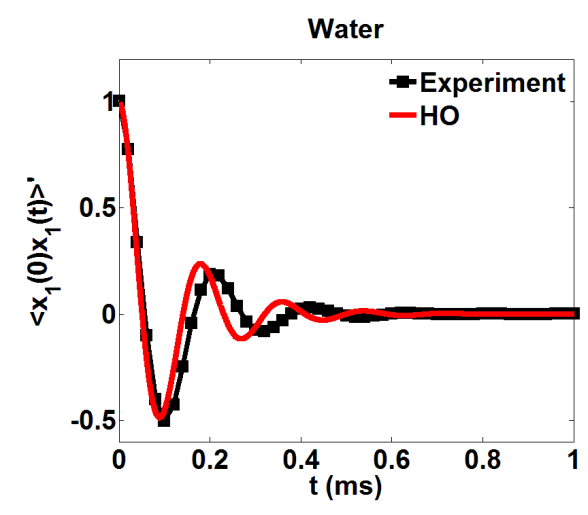

(c)

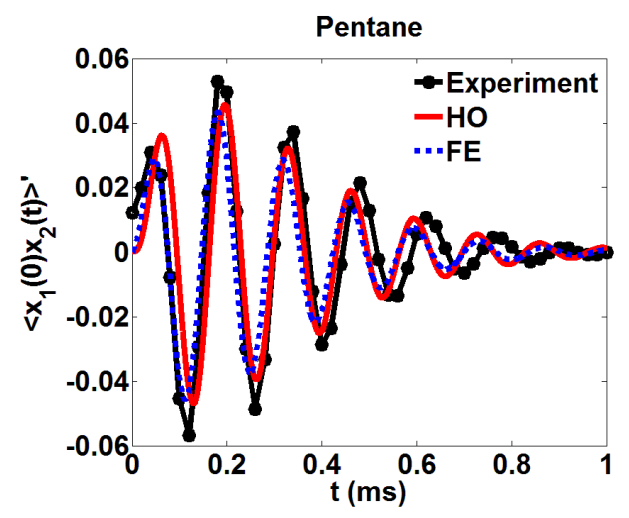

(b)

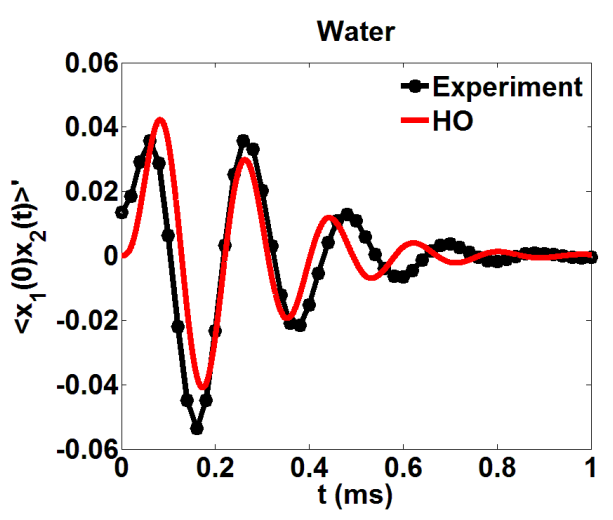

(d)

Figure 4-4: Comparison of HO model with experimental data. (a) and (b) auto- and crosscorrelations in n-pentane. (c) and (d) auto- and cross-correlations in water. Each plot shows the experimental results, the harmonic oscillator (HO) fit and the finite element (FE) model (using the known liquid viscosity and density). The ringdown from the $\mathrm{HO}$ and FE models have been used to yield the correlations in fluctuations using equations (2.4) and (2.5). These results are for a pair of commercial AFM cantilevers (ORC8 B: length $=200 \mu \mathrm{m}$, width $=40 \mu \mathrm{m}, \mathrm{k}=0.1 \mathrm{Nm}^{-1}$ ) separated by $8 \mu \mathrm{m}$ at $23^{\circ} \mathrm{C}$. 
Table 4-1: Fit parameters for HO model of the two-cantilever system. $\gamma_{\mathrm{a} \text {, heory }}$ is calculated from equation (29) in [43]. $m_{\mathrm{e}}$ is the fluid loaded mass of the cantilever in air, $m_{\mathrm{e}}=k_{\mathrm{c}} / \omega_{0}^{2}$, where $\omega_{0}$ is the resonant frequency in air. The viscosity, $\eta$, was measured using a controlled shear rheometer.

\begin{tabular}{|c|c|c|c|c|c|c|c|c|}
\hline \multirow{3}{*}{ Fluids } & \multicolumn{4}{|c|}{ Fit parameters } & \multicolumn{3}{c|}{ Derived quantities } & \multicolumn{1}{c|}{} \\
\cline { 2 - 8 } & $\gamma_{\mathrm{a}}[\mathrm{mg} / \mathrm{s}]$ & $\gamma_{\mathrm{c}}[\mathrm{mg} / \mathrm{s}]$ & $\omega_{\mathrm{r}} / \omega_{0}$ & $m[\mathrm{ng}]$ & $m / m_{\mathrm{e}}$ & $Q$ & $\gamma_{\text {a, heory }}[\mathrm{mg} / \mathrm{s}]$ & $\eta[\mathrm{mPa} . \mathrm{s}]$ \\
\hline Pentane & 0.51 & 0.077 & 0.38 & 44 & 2.4 & 4.1 & 0.58 & 0.22 \\
\hline Water & 1.1 & 0.14 & 0.28 & 79 & 4.6 & 2.5 & 1.62 & 0.94 \\
\hline
\end{tabular}

\section{Summary}

Through the experiments in laterally offset CFS I learned how fluid properties such as density and viscosity can affect cross-correlation function. Specifically, the fact that the cross-correlation function changes with fluid shows that the correlation is due to fluid coupling and not from mechanical coupling of the two cantilevers (e.g. due to sharing a same glass base). Furthermore, by comparison of theory and experiment these results validated equations (2.4) and (2.5). For the application of laterally offset CFS in microrheology the principal advantages of such a technique are: (1) it is minimally invasive: there are no "moving" parts, I simply monitor the thermally driven oscillations at equilibrium; (2) small sample volume; (3) the device is simple; (4) the cantilevers are fixed in space, so any number of solutions or suspensions can be washed across them for analysis; (5) the dimensions of the wet parts are only millimeters, so the device could be used as a dip probe; (6) alignment of the cantilevers is not critical (for $\sim$ micrometer changes in cantilever lateral or vertical separation there was only very small variation in both our experimental and simulation results). 


\section{Development of Colloidal Probe Correlation Force Spectroscopy: Case Study}

Particles are often found in a wide-range of natural and artificial materials such as blood, milk, paint, mud and soil, so the flow of particulate materials has long been of interest. The fluid flow near, and in the far field of a single colloidal particle has been studied by Stokes (1851) and Boussinesq (1903) [101-104]. The hydrodynamic interaction between two particles at near touching to large interparticle separations has also received considerable attention [105-108]. Many experiments have been performed on Brownian spheres for interparticle separations no less than about $1 \mu \mathrm{m}$ using optical tweezers [108-110], and interpreted via the fluctuationdissipation theorem.

The fluid motion surrounding a particle and in the far field obeys the Navier-Stokes equations $[43,102]:$

$$
-i \omega \operatorname{Re}_{\omega} \overrightarrow{\hat{u}}+\operatorname{Re} \overrightarrow{\hat{u}} \cdot \vec{\nabla} \overrightarrow{\hat{u}}=-\vec{\nabla} \hat{p}+\nabla^{2} \overrightarrow{\hat{u}}+\delta(r) \overrightarrow{\hat{F}}
$$

where $^{\wedge}$ denotes a Fourier transform, $\overrightarrow{\hat{u}}$ is the velocity field, $\omega$ is the angular frequency, $\hat{p}$ is the pressure field, the product $\delta(r) \overrightarrow{\hat{F}}$ is the force per unit volume exerted by the particle on the fluid at the origin $\vec{r}=0$, and $\delta$ is the Dirac delta function [111]. The Reynolds number is defined by $\operatorname{Re} \equiv U L / v$ and the frequency number is defined by $\operatorname{Re}_{\omega} \equiv L^{2} / T_{\mathrm{o}} v$, where $U$ and $T_{\mathrm{o}}$ are the characteristic velocity and time scale of particle motion in an otherwise stationary background fluid, and $L$ and $v$ are the characteristic length and kinematic viscosity. For most practical applications (e.g., the stability of colloidal solutions), $\operatorname{Re}$ and $\operatorname{Re}_{\omega}<O(1)$ and thus the majority of experimental and theoretical work has focused on the non-inertial form of equation (5.1) $[108,112-116]:$

$$
0=-\vec{\nabla} \hat{p}+\nabla^{2} \overrightarrow{\hat{u}}+\delta(r) \overrightarrow{\hat{F}}
$$


For Brownian particles in a potential field with a stiffness $k$ the velocity of oscillation can be represented as $U \equiv A \omega_{\mathrm{o}}$ where the amplitude is given by the equipartition theorem to be $A=\sqrt{k_{\mathrm{B}} T / k}$, the resonant frequency of oscillation is $\omega_{\mathrm{o}}=2 \pi / T_{\mathrm{o}}, k_{\mathrm{B}}$ is Boltzmann's constant, and $T$ is the absolute temperature. When the harmonic potential keeps the amplitude of thermal oscillations $A$ less than the characteristic length $L, \operatorname{Re}_{\omega} \gg>\operatorname{Re}[103]$, which results in the unsteady Stokes equation [43]:

$$
-i \omega \operatorname{Re}_{\omega} \overrightarrow{\hat{u}}=-\vec{\nabla} \hat{p}+\nabla^{2} \overrightarrow{\hat{u}}+\delta(r) \overrightarrow{\hat{F}}
$$

The relative importance of the unsteady term $\left(-i \omega \operatorname{Re}_{\omega} \overrightarrow{\hat{u}}\right)$ is determined by the frequency number $\operatorname{Re}_{\omega}$.

An example of a high frequency number technology is the electroacoustic determination of the zeta potential and the particle size [117], where particles are oscillated using a high frequency electric field or acoustic field. A full account of particle size and surface potential in this situation requires an understanding of the interparticle hydrodynamic and electrical interactions. Another example is fast microrheology where neglecting to include the unsteady inertial effects at very short time scales results in errors in fluid characterization [110, 118].

Even for a single sphere, the friction coefficient, ${ }_{\text {sph }}$, depends on the frequency number. The oscillation of the sphere results in an unsteady viscous boundary layer with depth $\xi_{\mathrm{v}}$ around the sphere [109]. The wake of this viscous layer has a penetration depth of order $\delta_{s} \equiv \sqrt{2 v / \omega}$ [103]. $\xi_{\mathrm{v}}$ is about $4 \delta_{s}$ for a sphere [109, 110]. Recently, Paul et al. [119] showed that for an oscillating cylinder $\xi_{\mathrm{v}}$ varies from $5 \delta_{s}$ to $20 \delta_{s}$ for frequency numbers ranging 1 to 100 . Comparing $\delta_{s}$ to the radius of the particle $a$, where $a$ is also the characteristic length scale for a single particle, two limiting cases are identified. One limiting case occurs when $\delta_{s}>>$. This can occur for low frequencies of oscillation where $\operatorname{Re}_{\omega} \ll<1$. In this case the drag on the single sphere is given by Stokes' formula $6 \pi \eta a$, where $\eta$ is the fluid dynamic viscosity. The other 
limiting case occurs at high frequencies where $\delta_{s} \leq a$ and $\operatorname{Re}_{\omega} \geq 1$. In this case the drag on the sphere increases to $[101,103]$ :

$$
\zeta_{\mathrm{sph}}=6 \pi \eta a\left(1+a / \delta_{s}\right)
$$

For the hydrodynamic interaction between two particles, the gap between the particles becomes important; I suggest that the relevant characteristic length is half the gap between the particles ( $\frac{1}{2} D$ ) (see Figure 5-1). By introducing a new length scale, a new frequency number is introduced, $\operatorname{Re}_{\omega} \equiv \frac{1}{2} D^{2} / \delta_{s}^{2}$, and it should be possible for the fluid inertial forces to be important for a single particle but not for a pair of particles (or vice versa). An AFM experiment, with its control of interparticle separation allows us to examine both the single-particle and the two particle regimes for the same particle. The purpose of the current paper is to use such measurements to understand the effect of fluid inertia for the two particle case. For the experiments described in this paper, a typical frequency is $4 \mathrm{kHz}$, which gives $\delta_{s} \cong 9 \mu \mathrm{m}$ in water. The radius is $a=15 \mu \mathrm{m}$, and $D$ is varied from a few nanometers (i.e., nearly-touching) to a few micrometers. Although the single particle-fluid interaction has a high frequency number $\frac{1}{2} \operatorname{Re}_{\omega} \equiv a^{2} / \delta_{s}^{2} \quad\left(\operatorname{Re}_{\omega} \cong 6\right)$, the two particle interaction will have a low frequency number, for $D \leq \sqrt{2} \delta_{s}$, if I use the characteristic length of $\frac{1}{2} D$. It is thus interesting to see whether the experimental interparticle hydrodynamic interaction can be accurately modeled with non-inertial formulae (equation $(5.2)$ ) $[105,106]$ even when a single particle is at high frequency number.

For the hydrodynamic interaction between two spheres, optical tweezers (OT) has proven to be a valuable technique. Recently, Atakhorrami et al. [109, 110] explored the unsteadiness of interparticle hydrodynamic interactions by pushing the time resolution of OT measurements to the $\mu$ s range. Neglecting particle inertia [79] and the restoring force due to the soft external harmonic potential, the fluid velocity field is related to the drag force via $\overrightarrow{\hat{u}}=-i \omega \alpha \overrightarrow{\hat{F}}$ where $\alpha$ is the interparticle hydrodynamic complex response function [107]. The power spectral density of the correlation of thermal fluctuations of the two spheres is given by:

$$
G_{12}=\int\left\langle x_{1}(t) x_{2}(0)\right\rangle e^{i \omega t} d t,
$$


where $x_{1}$ and $x_{2}$ are the thermal fluctuations of the position of the two spheres. $G_{12}$ is related to the imaginary part of the complex response function, $\alpha^{\prime \prime}$, via the fluctuation-dissipation theorem $[79,110]$. For motion parallel $(\|)$ to the centerline of the two spheres $\alpha^{\prime \prime}$ is [110]:

$$
\alpha_{\|}^{\prime \prime}(r, \omega)=\frac{1}{4 \pi \eta r \omega} Z,
$$

where $(4 \pi \eta r \omega)^{-1}$ is the steady interparticle hydrodynamic response, known as Oseen's response, and $Z$ is a correction due to unsteady fluid inertia $[109,110]$ :

$$
Z=\frac{[(1+z) \sin z-z \cos z] e^{-z}}{z^{2}},
$$

and $G_{12}$ is:

$$
G_{12}(r, \omega)=\frac{4 k_{B} T}{\omega} \alpha_{\|}^{\prime \prime}(r, \omega)
$$

where $r$ is the distance between the centers of the two particles (Figure 5-1) and $z=r / \delta_{s}$. For $z<<1$ (e.g., at a fixed interparticle separation $r$ and very low frequency $\omega$ ), $Z \cong 1$ and equation (5.6) reduces to the steady interparticle hydrodynamic response. At $r / \delta_{s}=1$ there is a $40 \%$ correction to the steady interparticle hydrodynamic response $(Z \cong 0.4)$. The interparticle hydrodynamic response approaches zero at $r / \delta_{s}>4(Z<<0.01)[109,110]$.

Optical traps use laser beams to exert an external harmonic potential with low stiffness $\left(10^{-6} \sim\right.$ $10^{-5} \mathrm{Nm}^{-1}$ ). The small restoring force and small particle inertia [79] makes it possible to relate $G_{12}$ to $\alpha^{\prime \prime}$ via equation (5.8). It is noted that the effective particle inertia is not only due to particle mass, but also the mass of the fluid displaced [101]. The displaced fluid mass is frequency dependent and is given by $\frac{4}{3} \pi \rho_{f} a^{3}\left(\frac{1}{2}+\frac{9 \delta_{s}}{4 a}\right)$ where $\rho_{f}$ is the fluid density. The fluid loaded mass (mass of the sphere plus the mass of the displaced fluid) is then:

$$
m_{\mathrm{sph}}=\left(\rho_{\mathrm{sph}}+\rho_{f}\left(\frac{1}{2}+\frac{9 \delta_{s}}{4 a}\right)\right) \frac{4}{3} \pi a^{3},
$$

where $\rho_{\mathrm{sph}}$ is the density of the sphere and $\frac{4}{3} \pi \rho_{\mathrm{sph}} a^{3}$ is the mass of sphere. I note the distinction between the fluid inertia $\left(-i \omega \operatorname{Re}_{\omega} \overrightarrow{\hat{u}}\right.$ term in equation (5.3)) and the inertia of the particle 
(equation (5.9)). The governing equation for the fluid is the Navier-Stokes equation and fluid inertia becomes significant at high $\mathrm{Re}_{\omega}$, while significant particle inertia appears in the correlation function between the two closely spaced spheres (equation (5.10)-(5.16)). The frequency dependent drag (equation (5.4)) and mass (equation (5.9)) restrict the permissible frequencies for the accurate application of equation (5.8). For high frequency measurements $(\sim$ $100 \mathrm{kHz}$ ), one has to choose small microparticles on the order $1 \mu \mathrm{m}$ in radius. The stiffness of the external potential should be kept very small $k_{\mathrm{c}}<20 \mathrm{mN} / \mathrm{m}$, while for higher stiffness equation (5.8) fails for any size of the microparticle. One shortcoming of optical traps is that the smallest interparticle separation is usually more than twice the wavelength of the laser beam $(\sim$ $1000 \mathrm{~nm})[43,108]$, so near-touching interparticle separations are inaccessible, which excludes interparticle collisions, where lubrication forces are greatest. This regime is important for interactions in concentrated suspensions, and during the collisions between particles that determine colloidal stability. Our approach has many similarities with the OT technique, but instead of applying the external potential using laser beams, I apply the potential by attaching an atomic force microscope (AFM) cantilever to each particle in the CFS. The cantilevers apply a relatively stiff external potential $\left(0.08 \mathrm{Nm}^{-1}\right)$ in one dimension (along the $x$ direction in Figure 5-1), and much stiffer potentials in the other dimensions (perpendicular to $x$ direction and for torsional motion). This restricts the Brownian motion to be most significant in one dimension and parallel to the centerline of the spheres. The potential exerted by AFM cantilevers is known to be harmonic, $F_{e x t}=-k_{\mathrm{c}} x$, where $x$ is the deflection from the equilibrium position. The particle inertia, as well as the restoring force, are important in our experiments and are included in the correlation function analysis for a more accurate measurement of the hydrodynamic interaction.

In our experiments, $\delta_{s}$ is fixed while $r$ (see Figure 5-1) is varied from the sum of the particles radii (near collision) to a few micrometers. I compared our experimentally calculated interparticle hydrodynamic interaction with available theories that were developed for the hydrodynamic interaction between two spheres at low Reynolds and frequency numbers [105, 106]. Our experimental data are in good agreement with these theories at near touching separations to about $2 \delta_{s}$, which supports our choice of characteristic length described above. I show that unsteady inertial effects are unimportant for interparticle separation $D$ less than about 
$2 \delta_{s}$. I attribute this effect to the overlapping $\delta_{s}$ surrounding each sphere. The underlying physics is that the elevated frictional forces at narrow gaps dominate fluid inertial effects. Therefore, I use the ratio of the separation between particles $D$ to $\delta_{s}$ to determine when the unsteady inertial effects are unimportant for interparticle interactions. This ratio is independent of the size of particles.

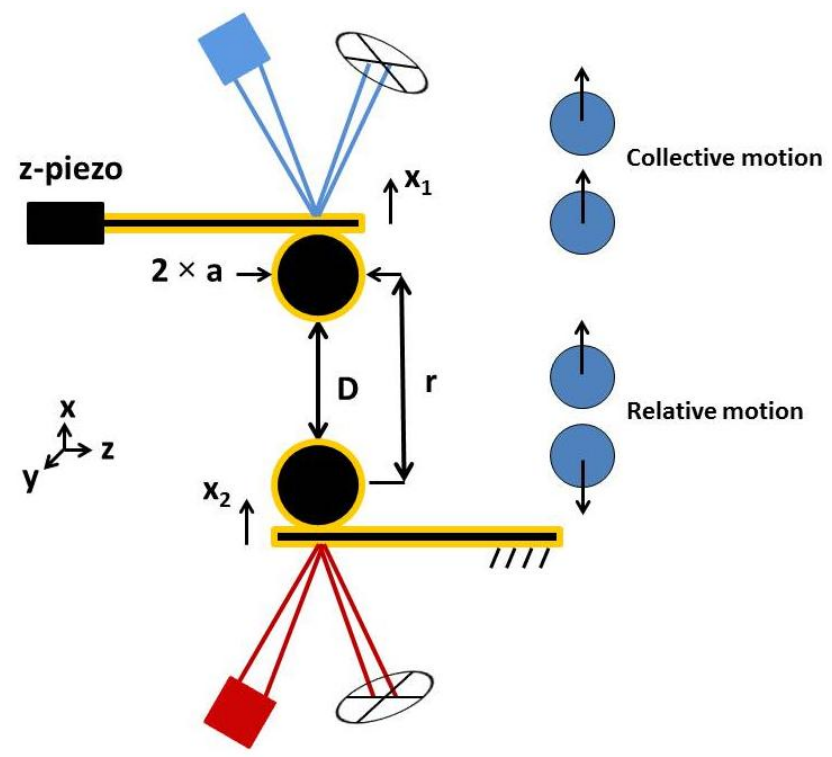

Figure 5-1: Schematic of the experiment. $a$ is the radius of a sphere, $D$ is the separation between the two spheres, and the distance between the spheres is $r=D+2 a$. The dimensionless centerto-center distance is $\rho=r / a . x_{1}$ and $x_{2}$ are the thermally-stimulated deflections of the cantilever-sphere assemblies that are detected by individual detection systems. The z-piezo in the top assembly is part of the commercial AFM and is used to vary the separation between the two spheres. In collective (symmetric) motion, the spheres move in the same direction while in relative (asymmetric) motion, they move in opposite directions.

\section{Materials and Methods}

Figure 5-1 shows a schematic of the experiment. Each microsphere, $30 \mu$ m-diameter (Duke Standards), was glued (epoxy) to the distal end of a different AFM cantilever, $200 \times 20 \times 1 \mu^{3}$ (ORC8-D, Bruker Inc.) The deflections of each of the cantilever-sphere assemblies, $x_{1}(t)$ and 
$x_{2}(t)$, were measured using the optical lever technique with a separate laser and photodiode for each assembly. The top detection system was part of a commercial AFM (MFP3D-Bio, Asylum Research, Oxford Instruments), which includes a superluminescent laser diode with an $860 \mathrm{~nm}$ wavelength. The customized bottom detection system includes a laser with a $680 \mathrm{~nm}$ wavelength (51nanoFCM, Schäfter + Kirchhoff $\mathrm{GmbH})$ and a photosensitive diode (QP50-618u-SD2, Pacific Silicon Sensor). The bottom detection system was mounted to the underneath of the MFP3D AFM plate. The two cantilever-sphere assemblies were put in a closed-fluid cell (Bioheater, Asylum Research, Oxford Instruments) and immersed in purified water at $\mathrm{pH} 7$ and at room temperature $\left(\sim 22^{\circ} \mathrm{C}\right)$. With this setup I conveniently exchange the solution in the fluid cell without disturbing the measuring system. The bottom cantilever was mounted at a slight angle $\left(\sim 5^{\circ}\right)$ relative to the top cantilever to prevent possible laser cross-talk between the two probes. The two micro-spheres were then brought in close proximity of each other using a micrometer translation stage. Using the top view microscope of the MFP3D and monitoring the deflection signal of the two assemblies I ensured that lateral offset between the spheres was minimal. The vertical separation between the spheres was varied from a few nanometers to tens of micrometers using the piezo-electric drive mechanism in the MFP3D.

Two different measurements were performed: a slow measurement of deflections while varying the separation, and a fast measurement at a constant separation. The slow measurement $(\sim 1$ $\mathrm{kHz}$ ) was used to capture the force-separation curve [120] with a typical approach velocity of 1 $\mu \mathrm{m} / \mathrm{s}$ (see Figure 5-2). Figure 5-2 shows that the force on approach is the same as the force when the spheres are pulled apart. This means that the lubrication force at this approach velocity is much smaller than the quasi-static forces. The force is roughly exponential with separation and is consistent with the existence of a double-layer repulsion [120] that extends up to $150 \mathrm{~nm}$ from contact. I measured the force as a function of piezo velocity and found that the lubrication force was negligible compared to the double-layer force in the range $10 \mathrm{~nm} / \mathrm{s}$ to $1000 \mathrm{~nm} / \mathrm{s}$. The fact that the deflection of one assembly is equal and opposite to the other assembly is consistent with correct calibration of the forces on each assembly. 


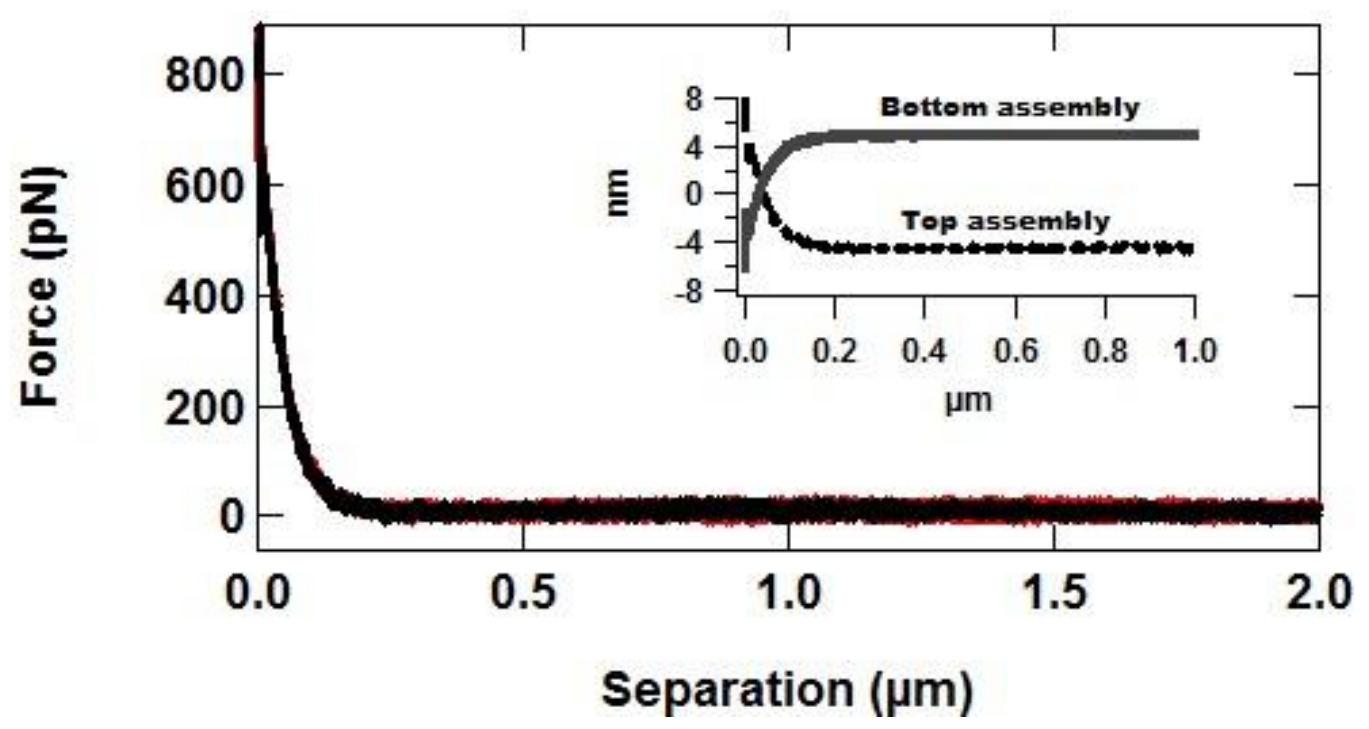

Figure 5-2: Approach and retract force-separation curves obtained by driving the top cantileversphere assembly at $1000 \mathrm{~nm} \mathrm{~s}^{-1}$ toward, and then away, from the bottom assembly. The overlay of the approach and retract curves shows that the lubrication force is very small compared to time-independent forces such as the double-layer forces. The inset shows the measured deflection of the top and bottom assemblies are equal and opposite.

The fast measurement $(1 \mathrm{MHz})$ is used to measure the thermal fluctuations of the two cantilevers, and from there, to determine the correlation in motion of the spheres. Here the assemblies were held at a fixed separation for $16 \mathrm{~s}$ while the time series of the deflection of each assembly were collected using a high frequency data acquisition card (NI PCI-6110, National Instrument, Irvine, CA). Within each assembly, the AFM cantilever applies an external harmonic potential with a stiffness of $0.08 \mathrm{~N} / \mathrm{m}$ on each sphere. This stiffness was measured in a separate experiment in air using the thermal method [121]. In our experiment, the resonant frequency of oscillations in water, $\omega_{0}=27000 \mathrm{rads}^{-1}$, and $v=10^{-6} \mathrm{~m}^{2} \mathrm{~s}^{-1}$. This results in $\mathrm{Re}_{\omega} \cong 6$ for a single particle and $\mathrm{Re} \cong 10^{-4}$.

\section{Analysis}

I used a Langevin theoretical framework to quantify our results. The time dependent position of the spheres is given by: 


$$
\left(\begin{array}{l}
m \ddot{x}_{1} \\
m \ddot{x}_{2}
\end{array}\right)+\left(\begin{array}{ll}
\zeta_{11} & \zeta_{12} \\
\zeta_{21} & \zeta_{22}
\end{array}\right)\left(\begin{array}{l}
\dot{x}_{1} \\
\dot{x}_{2}
\end{array}\right)+\left(\begin{array}{l}
k_{\mathrm{c}} x_{1} \\
k_{\mathrm{c}} x_{2}
\end{array}\right)=\left(\begin{array}{l}
f_{1}(t) \\
f_{2}(t)
\end{array}\right)
$$

where $m=k_{\mathrm{c}} / \omega_{0}^{2}=120 \mathrm{ng}$ is the fluid loaded mass, and $\zeta_{i j}$ is the friction tensor: $\zeta_{\mathrm{ii}}$ is the friction associated with the motion of a single particle, whereas $\zeta_{\mathrm{ij}, \mathrm{i} \neq \mathrm{j}}$ is the friction that depends on the relative motion of the two particles. For axisymmetric motion of two identical spheres, the friction tensor is symmetric with equal diagonal elements. $f_{i}(t)$ is the random Brownian force with two moments [108]:

$$
\left\langle f_{i}(t)\right\rangle=0,
$$

and,

$$
\left\langle f_{i}(t) f_{j}\left(t^{\prime}\right)\right\rangle=2 \zeta_{\mathrm{ij}} k_{B} T \delta\left(t-t^{\prime}\right) .
$$

Equation (5.10) can be expressed in terms of a symmetric coordinate, $X_{s}=\frac{1}{\sqrt{2}}\left(x_{1}+x_{2}\right)$, and an asymmetric coordinate, $X_{a s}=\frac{1}{\sqrt{2}}\left(x_{1}-x_{2}\right)$. The symmetric coordinate represents the motion of the center of mass of the two spheres, which I will call "collective" motion and the asymmetric coordinate represents the differential motion of the two spheres, which I will call "relative" motion (Figure 5-1). These coordinates are orthogonal and independent, i.e. $\left\langle X_{s}(t) X_{a s}(0)\right\rangle=0$. In terms of the new coordinates, equation (5.10) becomes:

$$
m \ddot{X}_{s}+\zeta_{s} \dot{X}_{s}+k X_{s}=f_{s},
$$

and,

$$
m \ddot{X}_{a s}+\zeta_{a s} \dot{X}_{a s}+k X_{a s}=f_{a s} .
$$

$\zeta_{s}$ and $\zeta_{a s}$ are the friction coefficients in the collective and relative coordinates respectively. Similarly, $f_{s}$ and $f_{a s}$ are the random Brownian forces sensed by the dual cantilever-sphere system in the collective and relative coordinates respectively. The noise spectra of the collective and relative coordinates are $G_{s}=4 \int_{0}^{\infty}\left\langle X_{s}^{2}(t)\right\rangle \cos (\omega t) d t$ and $G_{a s}=4 \int_{0}^{\infty}\left\langle X_{a s}^{2}(t)\right\rangle \cos (\omega t) d t$ which from fluctuation-dissipation theorem are [43]:

$$
G_{s}=\frac{4 k_{B} T \zeta_{s}}{\left(m \omega^{2}-k\right)^{2}+\zeta_{s}^{2} \omega^{2}}
$$


and

$$
G_{a s}=\frac{4 k_{B} T \zeta_{a s}}{\left(m \omega^{2}-k\right)^{2}+\zeta_{a s}^{2} \omega^{2}},
$$

respectively. It is noted that from equations (5.15) and (5.16), $G_{i j}=4 \int_{0}^{\infty}\left\langle x_{i}(t) x_{j}(0)\right\rangle \cos (\omega t) d t$ which for $i=j$ gives the self- (autocorrelation) noise spectrum of each assembly and for $i \neq j$ gives the mutual (cross-correlation) noise spectrum,

$$
G_{11}=\frac{\left(G_{s}+G_{a s}\right)}{2} ; \quad G_{12}=\frac{\left(G_{s}-G_{a s}\right)}{2} .
$$

In equations (5.15) and (5.16) all the parameters are known prior to the correlation measurement except $\zeta_{s}$ and $\zeta_{a s}$. These are obtained from the best fit of experimentally generated $G_{\mathrm{s}}$ and $G_{\mathrm{as}}$ to equations (5.15) and (5.16) respectively. Elements of the friction tensor (equation (5.10)) can be obtained via:

$$
\zeta_{11}=\frac{\zeta_{s}+\zeta_{a s}}{2} ; \quad \zeta_{12}=\frac{\zeta_{s}-\zeta_{a s}}{2}
$$

The friction coefficients $\zeta_{11}$ and $\zeta_{12}$ contain contributions from the sphere and the cantilever. However, for mutual friction $\zeta_{12}$, our interest is in the sphere contribution only, so I must separate the two effects. I assume that the contributions from the sphere and cantilever are additive: this assumption is based on the fact that the amplitude of thermal fluctuations is very small $(\sim 0.2 \mathrm{~nm}$ at the resonant frequency) compared to the length scale of the sphere $(a \sim 15$ $\mu \mathrm{m})$ and the cantilever $\left(w \sim 20 \mu \mathrm{m}\right.$, where $w$ is the cantilever width). For $\zeta_{12}$ the contribution from cantilever-cantilever hydrodynamic interactions is negligible because the cantilevers are far apart (at least $4 a \sim 60 \mu \mathrm{m}$ ): experiments with cantilevers that are two times wider showed that $G_{12}$ is negligible for two cantilevers without attached spheres at cantilever-cantilever separations of $\geq 60 \mu \mathrm{m}$. The other hydrodynamic contribution to $\zeta_{12}$ is due to one cantilever-sphere assembly and cantilever of the other sphere. The minimum separation between the objects in this case is $2 a \sim 30 \mu \mathrm{m}$. This contribution is also insignificant and can be approximated from the radial fluid velocity field around an oscillating cylinder (cylinder is the theoretical model for an oscillating cantilever [119]). At $30 \mu \mathrm{m}$ separation, the fluid velocity drops to less than $20 \%$ of 
the cantilever velocity (for $\operatorname{Re}_{\omega} \cong 6$ and $\omega=27000 \mathrm{rad} \mathrm{s}^{-1}$ ). For the previous case $(60 \mu \mathrm{m}$ separation) the fluid velocity drops to less than $7 \%$ of the cantilever velocity. Thus the fluid response or the correlation in thermal fluctuations of the two assemblies is dominated by spheresphere interactions and by the fluid spanning the gap between the two spheres.

Likewise, the friction coefficient for the motion of a single particle, $\zeta_{11}$, includes the effect of the cantilever, and I wish to subtract this contribution. I estimate the cantilever contribution using the expression for the drag on an infinite cylinder in a viscous fluid [43, 94]:

$$
\zeta_{\mathrm{cyl}}=\frac{\pi}{4} w^{2} L \rho_{f} \omega \Gamma^{\prime \prime},
$$

where $L$ is the cantilever length, and $\Gamma^{\prime \prime}$ is the imaginary component of the hydrodynamic function $\Gamma$ :

$$
\Gamma=1+\frac{4 i K_{1}\left(-i \sqrt{i \mathrm{Re}_{\omega}}\right)}{\sqrt{i \operatorname{Re}_{\omega}} K_{0}\left(-i \sqrt{i \mathrm{Re}_{\omega}}\right)},
$$

where $i=\sqrt{-1}$ and $K_{1}$ and $K_{0}$ are the Bessel functions [94]. Equation (5.20) has been successfully applied to cantilever oscillations in various experiments [91]. Equation (5.19) gives $\zeta_{\mathrm{cyl}}=0.6 \mu \mathrm{kg} \mathrm{s}^{-1}$. The friction on the sphere is then $\zeta_{\mathrm{sph}}=\zeta_{11}-\zeta_{\mathrm{cyl}}$. I checked the validity of this assumption by comparing theoretical and experimental values of $\zeta_{\mathrm{sph}}$ at high frequency number and at large separations where the theoretical value is known (equation (5.4)) [103]. There was only $14 \%$ error between theoretical predictions and the experimental value. From this point, our interest is in the friction on the sphere, so for simplicity I use $\zeta_{11}$ to describe the friction of single-sphere motion, i.e. our measured value of ${ }_{11}$ with the cantilever contribution subtracted. 

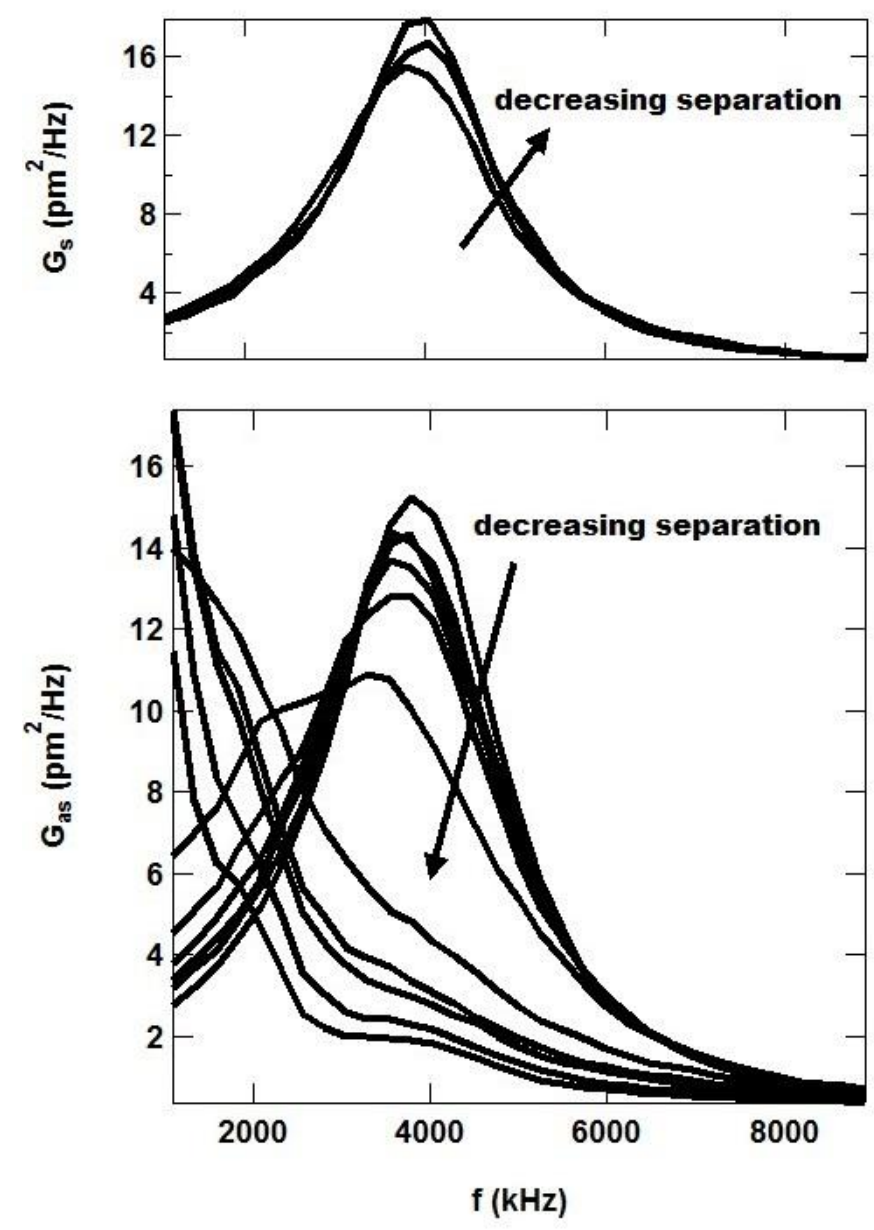

Figure 5-3: Power spectral density of the collective motion, $G_{s}$, and power spectral density of the relative motion, $G_{a s}$, at varying separations $D$. Only three separations are shown in the top graph, $D=\infty, 4 \mu \mathrm{m}$, and $10 \mathrm{~nm}$. Decreasing separations in the bottom graph are $D=\infty, 14 \mu \mathrm{m}$, $11 \mu \mathrm{m}, 7 \mu \mathrm{m}, 4 \mu \mathrm{m}, 2 \mu \mathrm{m}, 400 \mathrm{~nm}, 150 \mathrm{~nm}, 100 \mathrm{~nm}, 30 \mathrm{~nm}$, and $10 \mathrm{~nm}$.

\section{Results}

The thermally-stimulated deflections of two cantilevers each with a spherical particle attached were measured in water at varying separations between the spheres. The time series of the sum in deflections $\left(X_{s}=\frac{1}{\sqrt{2}}\left(x_{1}+x_{2}\right)\right)$ were used to calculate the power spectral density of symmetric (collective or center of mass) motion and the time series of the difference $\left(X_{a s}=\frac{1}{\sqrt{2}}\left(x_{1}-x_{2}\right)\right)$ in deflections was used to calculate the spectral density of the asymmetric (relative or differential) motion as described in the Analysis section (Figure 5-1). The results are shown in Figure 5-3. 
At infinite separation, $G_{\mathrm{s}}$ is very similar to $G_{\text {as }}$ and they both approach $G_{11}$ (the autocorrelation power spectral density). The symmetric motion is a weak function of separation: $G_{\mathrm{s}}$ increases very gradually as the separation decreases. In contrast, the asymmetric motion is a strong function of separation. This is an example of the power of cross-correlation measurements: the asymmetric signal is much more sensitive to the damping of the intervening medium than the symmetric signal, yet only the symmetric signal is available in conventional AFM measurements.

Data for $G_{\mathrm{s}}$ and $G_{\mathrm{as}}$ were fitted with equations (5.15) and (5.16) to obtain the values of $\zeta_{s}$ and $\zeta_{a s}$. The external potential is applied by the spring of constant stiffness, so I keep $k$ the same at all separations. I assume also that $m$ is independent of separation, so all the variation in $G_{\mathrm{s}}$ and $G_{\text {as }}$ arises from $\zeta_{s}$ or $\zeta_{a s}$. The variation in $\zeta_{s}$ or $\zeta_{a s}$ as a function of separation is shown in Figure 5-4. The abscissa on this graph is the dimensionless center-to-center distance, $\rho=r / a$. The slight increase in the amplitude of the symmetric spectral density is associated with the slight decrease in magnitude of the friction of collective motion $\left(\zeta_{s}\right)$. In keeping with the previous interpretation [112] I interpret this decrease in friction as an increase in the tendency of the fluid flow generated by one sphere to entrain the neighboring sphere when the spheres are closer to each other. The spectrum for $G_{\text {as }}$ is similar to a spectrum for an increasingly damped oscillator as the separation decreases. Fitted values for $\zeta_{a s}$ (Figure 5-4) quantitatively show the increasing damping at smaller separations. The increase in the friction of the relative motion ( $\left.\zeta_{a s}\right)$ at closer separations reflects the difficulty of squeezing the fluid into, and out of, the gap between the two spheres. 


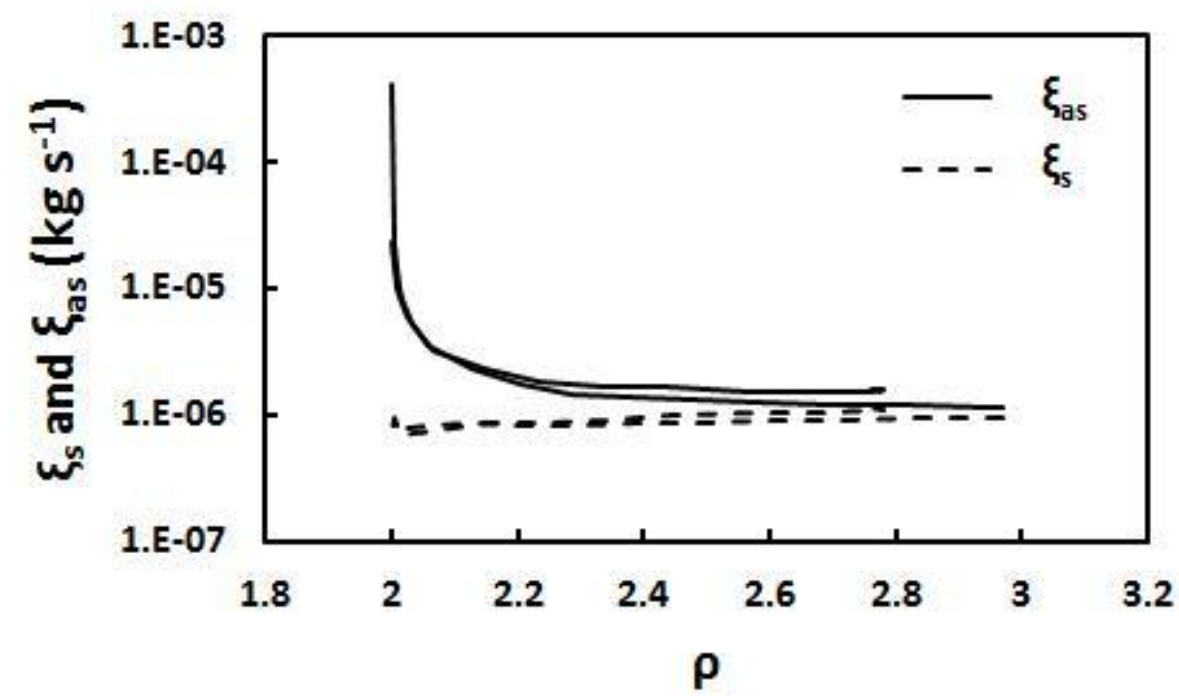

Figure 5-4: Values of $\zeta_{s}$ and $\zeta_{a s}$ obtained from fits to equation (5.15) and (5.16) using the data shown in Figure 5-3. Data is plotted as a function of the dimensionless separation $(\rho=r / a)$. Results are shown for two separate experiments.

$G_{12}$ is calculated from the difference between $G_{\mathrm{s}}$ and $G_{\text {as }}$ and is approximately zero at large separation (indicating that the particle motions are uncorrelated) and increases in magnitude with smaller separation (indicating that the particle motions are highly correlated) (see Figure 5-5). The lines in Figure 5-5 are plots of the analytical values calculated from equations (5.15)-(5.17) using the values of $\zeta_{s}$ and $\zeta_{a s}$ obtained from the fits to Figure 5-3. The increase in fluid coupling at smaller separations dampens the relative motion and enhances the collective motion (Figure 5-4). 


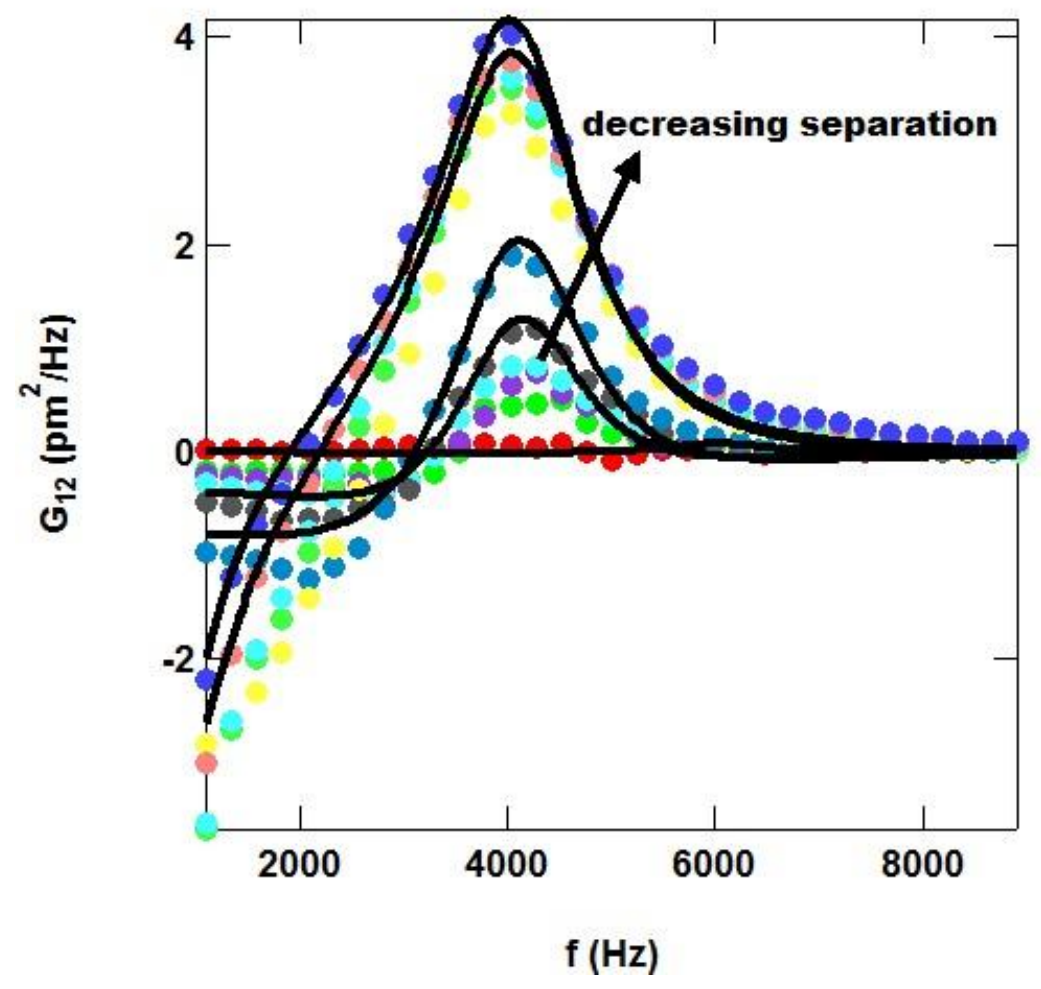

Figure 5-5: Cross-correlation spectral density, $G_{12}$, at varying separations $D$. Decreasing separations are $D=\infty, 14 \mu \mathrm{m}, 11 \mu \mathrm{m}, 7 \mu \mathrm{m}, 4 \mu \mathrm{m}, 2 \mu \mathrm{m}, 400 \mathrm{~nm}, 150 \mathrm{~nm}, 100 \mathrm{~nm}, 30 \mathrm{~nm}$, and $10 \mathrm{~nm}$. Solid lines show the quality of fits for separations $D=\infty, 4 \mu \mathrm{m}, 2 \mu \mathrm{m}, 30 \mathrm{~nm}$, and 10 $\mathrm{nm}$. At any given frequency, the magnitude of $G_{12}$ changes monotonically with separation.

A comparison to theoretical predictions of the friction coefficients is facilitated by normalizing the measured friction coefficient by the Stokes' drag, 6 $\pi \eta a$, as shown in Figure 5-6. Normalized data is signified by $\zeta_{11}^{*}$ and $\zeta_{12}^{*}$. The only high frequency number equation that I have is for $\zeta_{11}^{*}$ for a free sphere (equation (5.4)). From Figure 5-6 I see that our data agrees with this prediction within only $14 \%$ error, which is less than the systematic error in our experiment due to uncertainty in the spring constant and sphere radius. In the absence of a theory for interparticle hydrodynamic interactions that applies at high frequency number and with an interacting sphere, I compare our results to the theory for steady Stoke's flow (low frequency number) for the interaction between two spheres. For gaps much larger than the particle radius, Jeffrey and Onishi [106] give the following asymptotic expressions:

$$
\zeta_{11}^{*}=1+\frac{9}{4 \rho^{2}}+\frac{93}{16 \rho^{4}}+\frac{1197}{64 \rho^{6}},
$$




$$
-1 \times \zeta_{12}^{*}=\frac{3}{2 \rho}+\frac{19}{8 \rho^{3}}+\frac{387}{32 \rho^{5}} .
$$

These equations are plotted in Figure 5-6, and clearly do not fit the data well. They fail completely at small separations, as expected for a large separation approximation. At large separation, equation (5.21) fails to predict $\zeta_{11}^{*}$, but the value of $\zeta_{12}^{*}$ is reasonably accurate. I will return to the agreement between theoretical and experimental values of $\zeta_{12}^{*}$ later. For two spheres that are nearly touching Jeffrey gives the following asymptotic expressions (also from Ref. [106]):

$$
\begin{aligned}
\zeta_{11}^{*} & =\frac{1}{4(\rho-2)}-\frac{9}{40} \ln (\rho-2)+0.99536-\frac{3(\rho-2)}{112} \ln (\rho-2)+0.19(\rho-2), \\
-1 \times \zeta_{12}^{*} & =\frac{1}{4(\rho-2)}-\frac{9}{40} \ln (\rho-2)+0.35022-\frac{3(\rho-2)}{112} \ln (\rho-2)+0.13185(\rho-2) .
\end{aligned}
$$

As shown in Figure 5-6, these expressions agree with our data for $\zeta_{11}^{*}$ and $\zeta_{12}^{*}$ at small separations despite the fact that they are low frequency number predictions and $\mathrm{Re}_{\omega} \cong 6$ for a single particle in our experiments. Our explanation for the observed agreement between data at high frequency number and theory at low frequency number is that $\zeta_{12}^{*}$ is dominated by flow in the thin region between the spheres. In the wake of rotational flow, in a layer of depth $\delta_{s}$, the fluid response is similar to steady Stokes flow $[109,110]:$ as $r / \delta_{s} \rightarrow 0$ there is a steady response and as $r / \delta_{s} \rightarrow 1$ there is a $40 \%$ correction to the steady response. Therefore, for interparticle separations shorter than twice the penetration depth $\delta_{s}$, the propagation of stress in the fluid is effectively instantaneous. The dominance of the interparticle hydrodynamic interactions by the thin fluid region spanning the gap of the two spheres results in a quantitative agreement of equation (5.24) with our experimental data at all separations and equation (5.22) for $\zeta_{12}^{*}$ at large separations. 

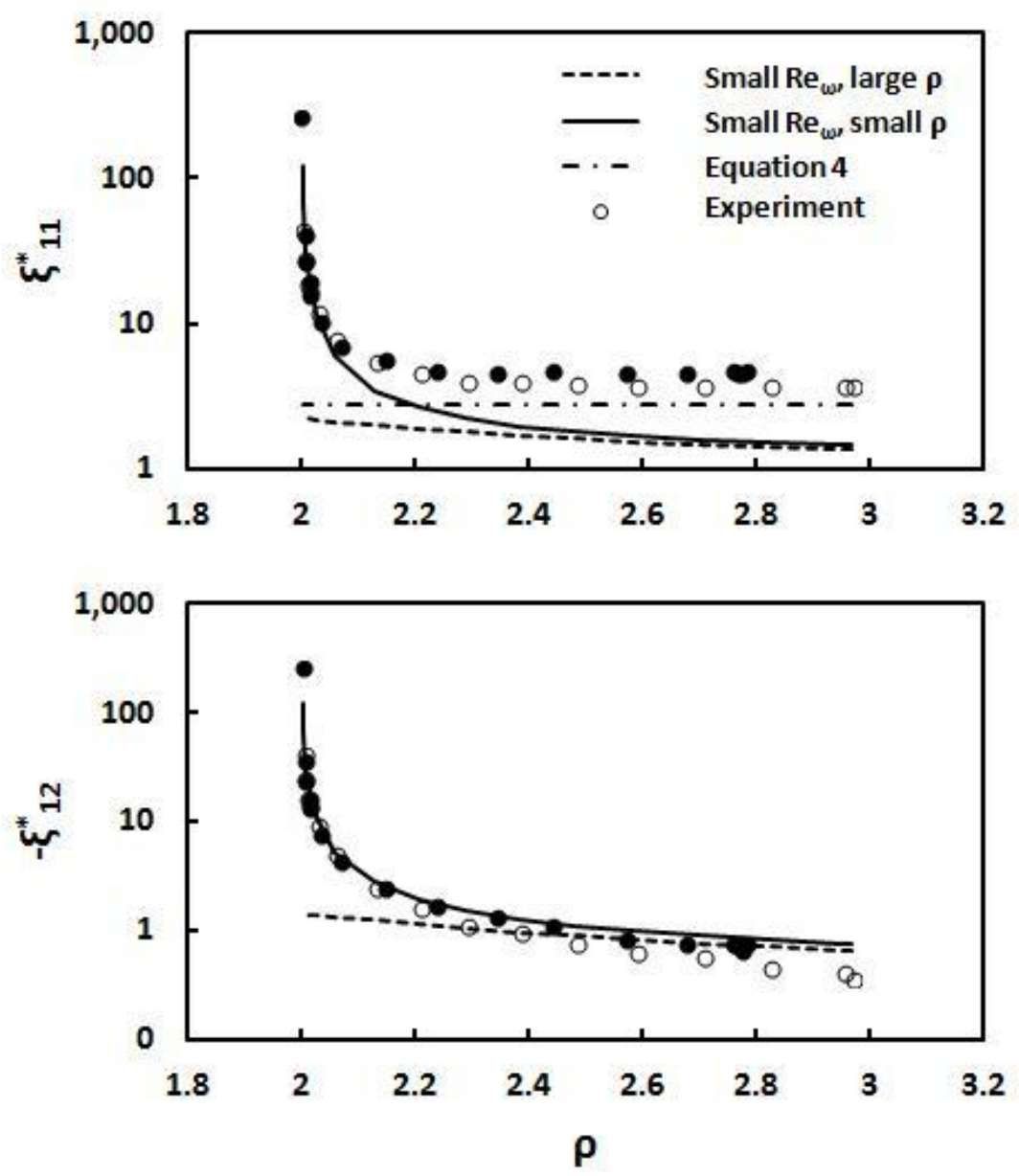

Figure 5-6: Friction of single sphere motion $\zeta_{11}^{*}$, and friction of mutual motions of two spheres, $\zeta_{12}^{*}$, as a function of dimensionless separation $\rho=r / a$. (Note, that I actually plot ${ }_{12}^{*}$.) Filled and hollow circles represent two different experimental data sets. Experimental data are compared with expressions (5.21) and (5.22) for small frequency number $\operatorname{Re}_{\omega}$ and large separations $\rho$ and expressions (5.23) and (5.24) for small frequency number $\operatorname{Re}_{\omega}$ and small separations ${ }^{6}$. Equation (5.4) is the high frequency number friction on a free sphere. Data are normalized by $6 \pi \eta a$.

As described in the Introduction, I account for the experimental observation that the effects of fluid inertia do not appear to be important as small separations by introducing the characteristic length of $D / 2$ in the calculation of $\operatorname{Re}_{\omega}$. The frequency number can thus be reformulated to give $\operatorname{Re}_{\omega} \equiv \frac{1}{2} D^{2} / \delta_{s}^{2}$ where the effect of the fluid density, viscosity, and the frequency of oscillation is included in $\delta_{s}$. This definition implies that at a fixed frequency of oscillation, the 
contribution of fluid inertia grows as the gap between the particles grows and for a fixed interparticle separation, the fluid inertia becomes more important at a higher frequency of oscillation. The properties of the fluid, in which the particles are dispersed, are also important: the lower the fluid kinematic viscosity the higher the inertial response. So, for example, the inertial response of water is higher than for air between two colloidal particles. Using the above definition of $\operatorname{Re}_{\omega}$ for interparticle hydrodynamics all our experiments presented here are in the regime of $\operatorname{Re}<1$ in the region between the particles.

\section{Discussion}

Comparison to Optical Tweezers Experiments. Equation (5.8) assumes that the particle inertia and the external restoring force are insignificant when compared with the viscous drag force (i.e., a highly over damped oscillator) and therefore is applicable for analysis of optical tweezers experiments. In contrast, the particle inertia and the restoring force are important in the two cantilever-sphere experiment, and thus equation (5.8) is inapplicable. Figure 5-7 shows that equation (5.8) fails to predict the interparticle hydrodynamic response in our experiments. Thus, particle inertia and the restoring force cannot always be ignored when discussing hydrodynamic interactions between particles. 


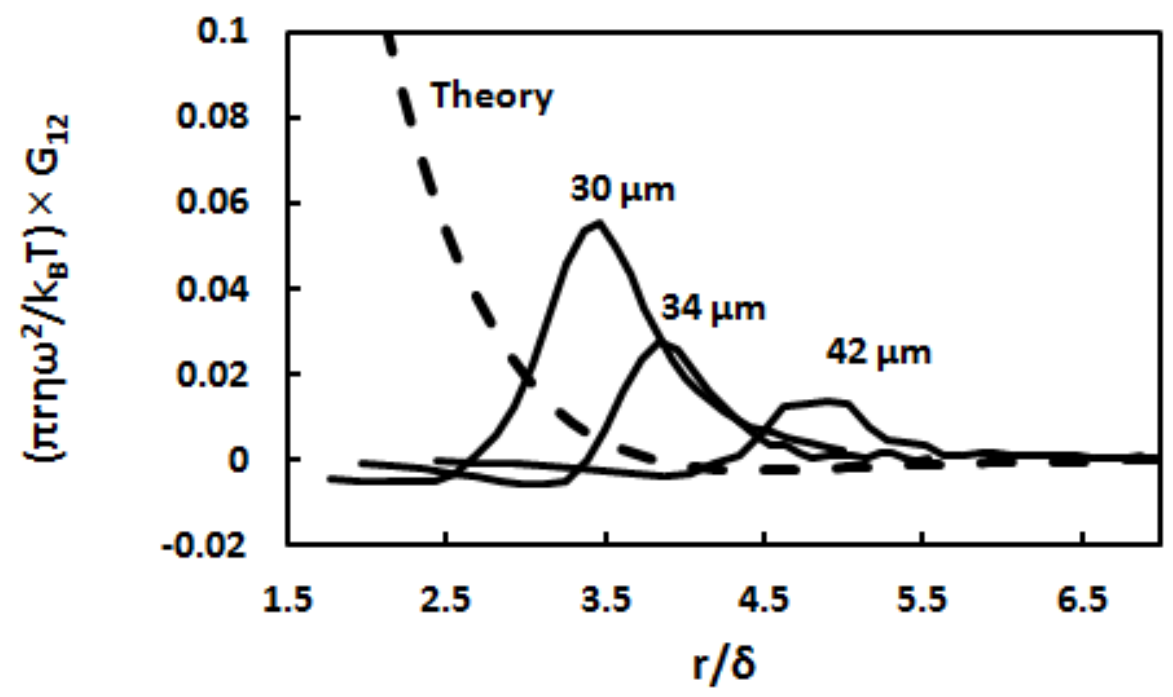

Figure 5-7: Normalized cross-correlation function $\left(\pi r \eta \omega^{2} / k_{B} T\right) G_{12}$ compared for several separations with theoretical expression $e^{-z} / z^{2}[(1+z) \sin (z)-z \cos (z)]$ in equation (5.6) and plotted versus the dimensionless ratio $z=r / \delta$. For each experimental data set, $r$ and $v$ are fixed, so $r / \delta$ is a function of frequency only.

Comparison to Conventional Drainage Experiments. A conventional drainage experiment is often used to obtain the lubrication force and thus the friction coefficient acting on a sphere approaching another fixed object such as a plate or sphere $[122,123]$. In such experiments, the fluctuations in the deflection of the AFM cantilever are usually low-pass filtered and thus the fast fluctuations and time-dependent effects are excluded [43, 94]. Two of the major weaknesses of this approach are avoided in the experiments described here. First, the conventional drainage experiment measures the sum of the quasistatic force and the lubrication force, sometimes making it difficult to resolve the lubrication force. For example the quasistatic double-layer force in Figure 5-2 completely obscures the lubrication force, whereas the lubrication force can be easily be separated and resolved as shown here from the analysis of the Brownian motion. Second, the conventional drainage experiment suffers from significant interfering hydrodynamic contributions due to the interaction between the cantilever and the bottom plate. This problem is largely solved in this work by supporting the bottom sphere on a cantilever that is small and antiparallel to the top cantilever such that the coupling between opposing cantilevers is weak. 
This type of improvement could also be made to the conventional drainage experiment, for example, by attaching the lower sphere to the edge of a step (Figure 5-8).
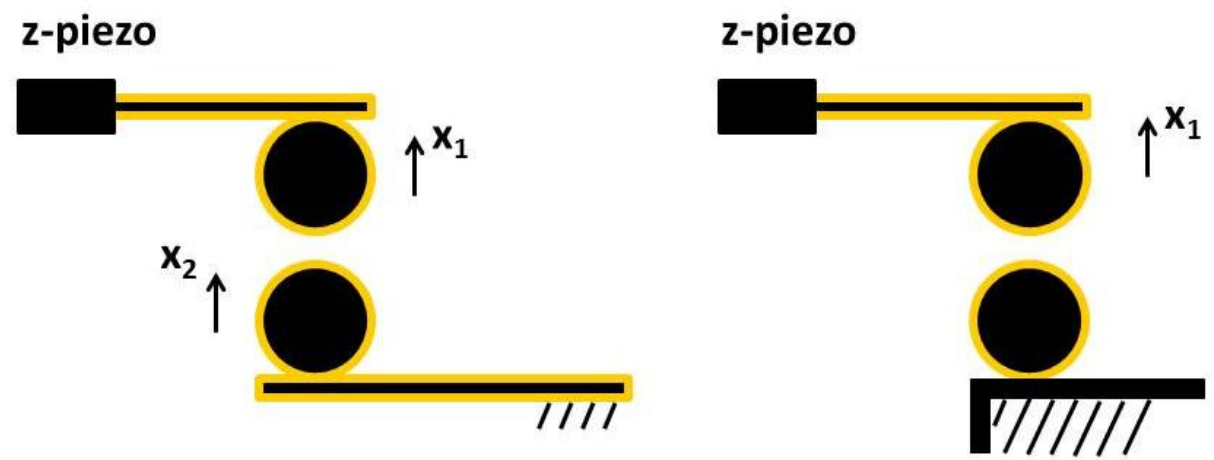

Figure 5-8: The current experiment reduces the hydrodynamic coupling between cantilevers by keeping them well separated and antiparallel (left). A conventional drainage experiment could achieve a similar effect by attaching the bottom sphere to a step edge (right).

\section{Summary}

A direct measurement of the hydrodynamic interactions between two micro-spheres for the conditions of low Reynolds number and high frequency number was also presented in this chapter using vertically offset CFS. At small separations, the friction of the relative motion increases dramatically because of the extra difficulty in squeezing the liquid out of a small film. In contrast, the friction of the collective motion decreases slightly because each particle is dragged in the wake of the other particle. At small separations between the spheres $\left(D<2 \delta_{s}\right)$ the measured value of the interparticle friction is well predicted by low frequency number theory (Jeffry's expression from Ref. [106]). This suggests that I use a frequency number for the interparticle hydrodynamic interaction that includes an explicit dependence on the separation of the spheres, for example, $\operatorname{Re}_{\omega} \equiv \frac{1}{2} D^{2} / \delta_{s}^{2}$. Thus, inertial effects should be included for $\operatorname{Re}_{\omega}>1 \Rightarrow D / \delta_{s}>1.4$. The physical interpretation of this relation is that when the separation between the spheres is less than about two times the wake of the unsteady viscous boundary layer, then the flow is effectively a Stokes' flow. This is because in narrow gaps, frictional forces dominate fluid inertial effects. As the separation $D$ increases, the low frequency number does not accurately describe the experiment, particularly for the friction of single particle 
motion. This is explained as a diminishing effect of confinement of the liquid between particles. The behavior of the particle at large separation asymptotes to that predicted by Stokes for a single particle at high frequency number.

This chapter also serves as a validation experiment for measurement of friction via crosscorrelation between two cantilevers in the CFS. The data was shown to agree with theory and therefore the technique can be used for measuring molecular friction in single molecule experiments. 


\section{Correlation Force Spectroscopy for Single Molecule Measurements}

This section starts with showing experimental measurements of correlations in thermal fluctuations of a cantilever pair as a function of separation between the cantilevers in water in the vertically offset CFS. Schematic of vertically offset CFS is shown Figure 6-1. Understanding the motion of the cantilever in water is a necessary precursor to understanding the motion of an added molecule spanning the gap between the cantilevers. I then characterize the hydrodynamic damping using a simple harmonic oscillator (HO) model. The HO model allows me to parameterize fluid properties, and in the future will allow the addition of parameters of the straddling polymer (stiffness, damping) that can be used to characterize the straddling molecules.

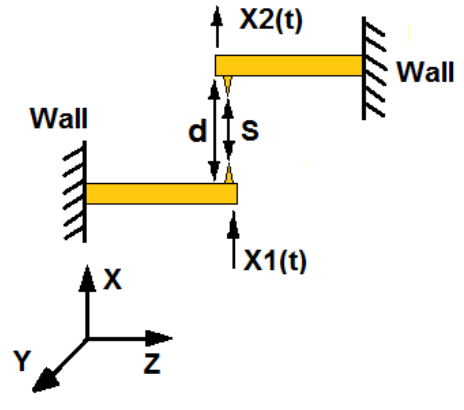

(a)

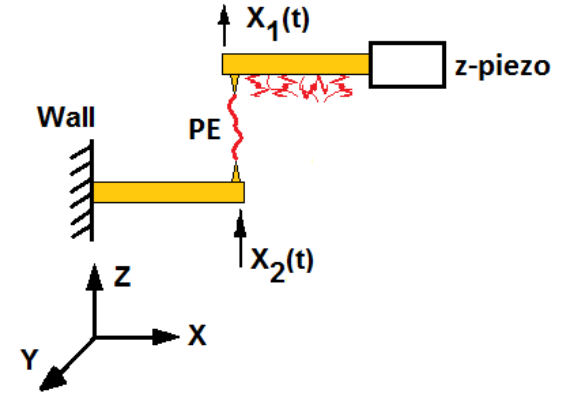

(b)

Figure 6-1: (a) Schematic of antiparallel vertically offset cantilevers. Tip height is $h$, thus $d=s+$ 2h. (b) Schematic figure showing a molecule being stretched between the tips.

\section{Effect of the Distance between Cantilever Tips}

The properties of a single molecule depend on the extension of the molecule, which requires a change in separation of the cantilevers. The first step in this experiment is to analyze the behavior of the cantilevers in water without a spanning molecule. 
The autocorrelations and cross-correlations of the deflections of two cantilevers at various separations in water are shown in Figure 6-2. The autocorrelation is a very weak function of separation. In contrast, the magnitude of the cross-correlation increases monotonically as the tips are brought closer together. This is expected: the fluid coupling is stronger when the tips are closer together. A similar effect has been observed for two particles using the optical tweezers technique [112]. The change in cross-correlation with separation also demonstrates that the observed correlation arises from the fluid coupling in the tiny gap between the cantilevers, and not from a spurious external driving of the entire device by a noise source in the lab.

The cross-correlation does not change much at smaller cantilever separations $(d=6.5-7.2 \mu \mathrm{m})$, which corresponds to smaller tip separations $(s=318-1000 \mathrm{~nm})$. This can be explained by the fact that the cantilevers have a large overlap $8 \mu \mathrm{m} \times 40 \mu \mathrm{m}$ (Figure 2-4) compared to the tip size (4 $\mu \mathrm{m})$, and are well within one Stoke's length of each other, which means that the flow around the cantilevers interacts strongly. The size of the unsteady viscous boundary layer around the oscillating cantilever is approximately given by the Stokes length [101], $\delta_{\mathrm{s}}=\sqrt{v / \omega_{r}}$ where $v$ is the kinematic viscosity and $\omega_{r}$ is the resonant frequency in the fluid. For our apparatus the Stokes length is approximately $14 \mu \mathrm{m}$ for ORC8-B cantilever in water. When the two cantilevers are separated by only $7 \mu \mathrm{m}$, it is not surprising that the hydrodynamics is dominated by the much larger cantilevers and not the tips.

The important point is that the water contribution to the cantilever coupling is very weakly dependent on the tip-tip separation. The water coupling of the cantilevers is a "noise" in the single molecule measurements and it is useful that this can be held constant in a model while molecules are subject to different extensions and compressions by the tips.

Figure 6-2 also shows that during the time span of 0 to $0.5 \mathrm{~ms}$ there are several in-phase and outof-phase correlations in thermal fluctuations of the two cantilevers. This behavior is different from the behavior of two particles trapped in an optical trap, where only one anti-correlation can be seen over 0 to $4 \mathrm{~ms}$ [108] because of the lower $Q$. This shows the capability of CFS to give more detailed information over a shorter time span. The separations shown in Figure 6-2 were 
obtained by extending the z-piezo so that the tips touched each other (which defines zero), then retracting the piezo to the desired position, measuring the auto- and cross-correlation, and then again extending the z-piezo to touch the tips. Each value of $s$ is the average distance travelled before and after the correlations are measured. Typically, the distance before and after varied by about $200 \mathrm{~nm}$, which shows the extent of the drift during the measurement time, $50 \mathrm{sec}$.

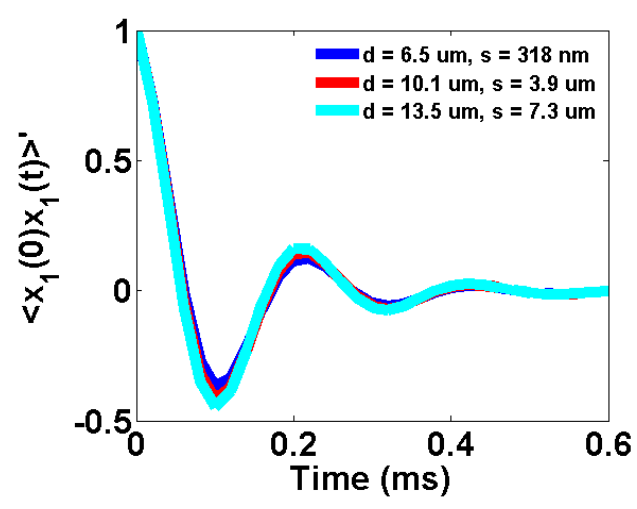

(a)

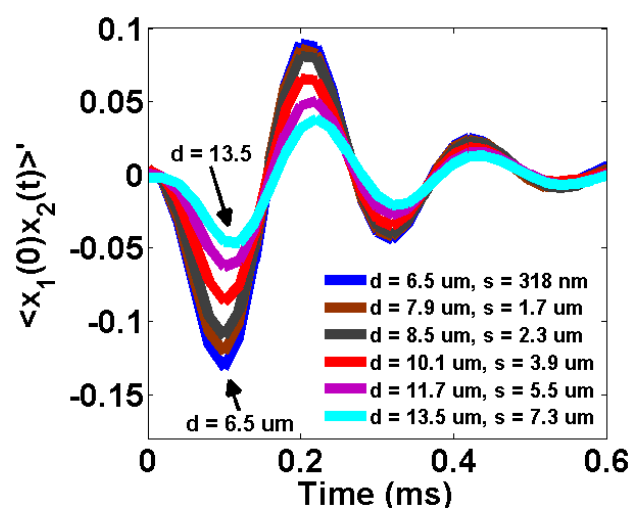

(b)

Figure 6-2: (a) Autocorrelation and (b) cross-correlation of equilibrium fluctuations in cantilever deflection in water at $23^{\circ} \mathrm{C}$. A series of measurements is shown for various tip-tip separations, $s$ $=318-7300 \mathrm{~nm}$, which corresponds to a cantilever-cantilever separation, $d=6.5-13.5 \mu \mathrm{m}$. $\mathrm{d}=$ $6.5 \mu \mathrm{m}$ and $\mathrm{d}=13.5 \mu \mathrm{m}$ are labeled and the rest of separations are in sequential order. Commercial AFM cantilevers are used (ORC8 B: length $=200 \mu \mathrm{m}$, width $=40 \mu \mathrm{m}, \mathrm{k}=0.1 \mathrm{Nm}^{-}$

${ }^{1}$ ). The prime on the left axis indicates normalized correlation function. Note that the autocorrelation data for different separations almost overlay: there is a slight decrease in amplitude at smaller separations. The cross-correlation increases monotonically as the separation decreases. Fluctuations are collected at $50 \mathrm{kHz}$ for $50 \mathrm{sec}$. using the Asylum Research MFP3D controller. Discrete points have been represented as a line for clarity.

\section{Harmonic Oscillator Modeling of Vertically Offset Correlation Force}

\section{Spectroscopy}

I have modeled the vertical offset experiment as a pair of coupled simple harmonic oscillators as described above for the lateral offset experiment. The significant difference is that for the lateral offset experiment, the damping occurred for in phase (symmetric) motion, whereas for the vertical offset, I set damping for out of phase (antisymmetric) motion (see Figure 6-3). In this configuration, the damper applies force when the two lumped massed displace antisymmetrically, i.e. one upward and the other downward. 


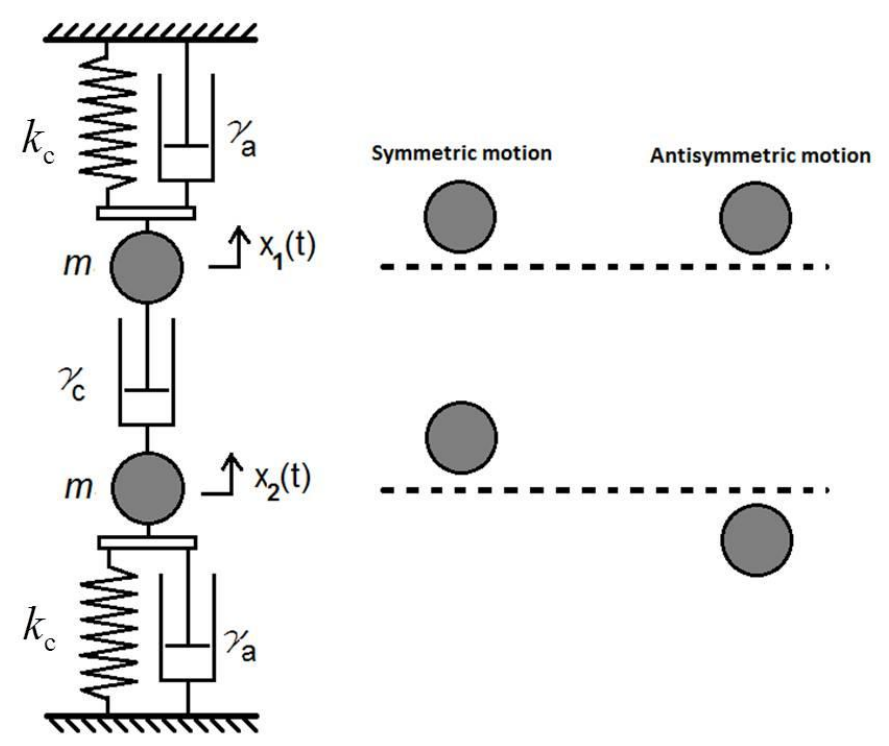

Figure 6-3: Schematic of a cantilever pair modeled as a mass-spring-damper system. $m$ is the effective fluid loaded mass of the cantilever, $k_{\mathrm{c}}$ is the spring constant, and $\gamma_{\mathrm{a}}$ is the coefficient of fluid damping on an individual cantilever. $\gamma_{\mathrm{c}}$ is the coefficient of fluid damping due to the fluid spanning the gap between the two cantilevers. The plates shown in the schematic are massless.

The dynamic equations of motion for the two masses are as follows:

$$
\begin{aligned}
& \ddot{X}_{1}+\left(\frac{\gamma_{\mathrm{a}}}{m}+\frac{\gamma_{\mathrm{c}}}{m}\right) \dot{X}_{1}-\frac{\gamma_{\mathrm{c}}}{m} \dot{X}_{2}+\omega_{r}^{2} X_{1}=0, \\
& \ddot{X}_{2}+\left(\frac{\gamma_{\mathrm{a}}}{m}+\frac{\gamma_{\mathrm{c}}}{m}\right) \dot{X}_{2}-\frac{\gamma_{\mathrm{c}}}{m} \dot{X}_{1}+\omega_{r}^{2} X_{2}=0 .
\end{aligned}
$$

The initial conditions are the same as lateral offset experiment. The symmetric and antisymmetric modes are also defined in the same way: $X_{\mathrm{s}}=X_{1}+X_{2}, X_{\mathrm{a}}=X_{1}-X_{2}$. The motion of the cantilevers are then $X_{1}=\left(X_{\mathrm{s}}+X_{\mathrm{a}}\right) / 2$ and $X_{2}=\left(X_{\mathrm{s}}-X_{\mathrm{a}}\right) / 2$. The solution to equations (6.1) and (6.2) are: 


$$
\begin{aligned}
X_{1}= & \frac{\exp \left(-\frac{\gamma_{\mathrm{a}}}{2 m} t\right)}{2}\left[\cos \left(\sqrt{\omega_{\mathrm{r}}^{2}-\left(\frac{\gamma_{\mathrm{a}}}{2 m}\right)^{2}}\right)+\frac{\frac{\gamma_{\mathrm{a}}}{2 m}}{\sqrt{\omega_{\mathrm{r}}^{2}-\left(\frac{\gamma_{\mathrm{a}}}{2 m}\right)^{2}}} \sin \left(\sqrt{\omega_{\mathrm{r}}^{2}-\left(\frac{\gamma_{\mathrm{a}}}{2 m}\right)^{2} t}\right)\right] \\
+ & \frac{\exp \left(-\omega_{\mathrm{d}} t\right)}{2}\left[\cos \left(\sqrt{\omega_{\mathrm{r}}^{2}-\omega_{\mathrm{d}}^{2}} t\right)+\frac{\omega_{\mathrm{d}}}{\sqrt{\omega_{\mathrm{r}}^{2}-\omega_{\mathrm{d}}^{2}}} \sin \left(\sqrt{\omega_{\mathrm{r}}^{2}-\omega_{\mathrm{d}}^{2}} t\right)\right], \\
X_{2}= & \frac{\exp \left(-\frac{\gamma_{\mathrm{a}}}{2 m} t\right)}{2}\left[\cos \left(\sqrt{\omega_{\mathrm{r}}^{2}-\left(\frac{\gamma_{\mathrm{a}}}{2 m}\right)^{2}}\right)+\frac{2 m}{\sqrt{\omega_{\mathrm{r}}^{2}}-\left(\frac{\gamma_{\mathrm{a}}}{2 m}\right)^{2}} \sin \left(\sqrt{\omega_{\mathrm{r}}^{2}-\left(\frac{\gamma_{\mathrm{a}}}{2 m}\right)^{2}} t\right)\right] \\
& -\frac{\exp \left(-\omega_{\mathrm{d}} t\right)}{2}\left[\cos \left(\sqrt{\omega_{\mathrm{r}}^{2}-\omega_{\mathrm{d}}^{2}} t\right)+\frac{\omega_{\mathrm{d}}}{\sqrt{\omega_{\mathrm{r}}^{2}-\omega_{\mathrm{d}}^{2}}} \sin \left(\sqrt{\omega_{\mathrm{r}}^{2}-\omega_{\mathrm{d}}^{2}} t\right)\right] .
\end{aligned}
$$

In the analysis of vertical offset experiments using the $\mathrm{HO}$ model, $\gamma_{\mathrm{a}}$ and $\omega_{r}$ are fit to the autocorrelation function when the two cantilevers are at large separation, where all damping is attributed to $\gamma_{\mathrm{a}} \cdot \gamma_{\mathrm{c}}$ is then fit for each tip to tip separation of the cantilevers while $\gamma_{\mathrm{a}}$ and $\omega_{r}$ are kept constant.

The best fit for $\gamma_{\mathrm{c}}$ was obtained by minimizing the deviation squared between the measured cross-correlation and the cross-correlation from the $\mathrm{HO}$ model over the range 0 to $1 \mathrm{~ms}^{4}{ }^{4}$ The HO fit is shown in Figure 6-4 together with the experimental data for water at several cantilevercantilever distances (see Figure 6-1). The agreement between theory and experiment is very good at the small separations, which is the important region in single molecule spectroscopy. Thus I have set the base model to which the properties of a straddling molecule can be added in future single-molecule studies. The absolute values of the fit parameters are also of interest. First, the fitted value of $\gamma_{\mathrm{a}}=15 \times 10^{-7} \mathrm{~kg} / \mathrm{s}$ (obtained at large separation and held constant for other separations), is similar to the value obtained, $\gamma_{\mathrm{a}}=17 \times 10^{-7} \mathrm{~kg} / \mathrm{s}$, from a theoretical

\footnotetext{
${ }^{4}$ I have subsequently determined that it is better to fit to the data in the frequency domain than in the time domain. The reason is that I am able to filter out low-frequency noise simply by not fitting to the low frequency data. The low frequency noise affects the entire data in the time domain, so it is more difficult to understand its deleterious influence.
} 
treatment of a cylinder using frequency-dependent damping (equation (29) in [43]). The fits to the hydrodynamic coupling as a function of separation are shown in Figure 6-5-a. For small separations $(s=318-1000 \mathrm{~nm}) \gamma_{\mathrm{c}}$ is almost constant and these are the separations of interest in molecule force spectroscopy; at these separations $\gamma_{c}$ is about half of $\gamma_{a}$. This result has important implications for measurements of "molecular friction" or "damping" in single molecule force measurements. If only one cantilever is used, $\gamma_{\mathrm{a}}$ is the background damping against which the molecular damping must be measured. If two cantilevers are used, $\gamma_{\mathrm{c}}$ is the background damping, which in this case is a factor of two or more smaller. At separations greater than $1000 \mathrm{~nm}, \gamma_{\mathrm{c}}$ diminishes monotonically and approximate linearly with separation until it is about an order of magnitude smaller than $\gamma_{\mathrm{a}}$ (at a separation of about $13 \mu \mathrm{m}$ ).

The magnitude of the force noise amplitude, $F_{i j}$, can be estimated from the auto- and crosscorrelations [43]:

$$
F_{i j}=k_{\mathrm{c}} \sqrt{\left|\left\langle x_{i}(0) x_{j}(t)\right\rangle\right|_{\max }},
$$

where $i=j$ for the single cantilever measurement. The noise amplitude will depend in general on the separation between the cantilevers and the spring constant. For this experiment in water, the spring constant is $\sim 0.1 \mathrm{Nm}^{-1}$ and the maximum amplitude of the cross correlation is $\sim 0.0051$ $\mathrm{nm}^{2}$, so $F_{12} \sim 8 \mathrm{pN}$ when the cantilevers are separated by $d=6.5 \mu \mathrm{m}(s=318 \mathrm{~nm})$. For the same cantilever the thermal noise is about $F_{11} \sim 20 \mathrm{pN}$, thus the thermal noise is about 2.5 times smaller in the two cantilever experiment. Figure 6-5-b shows $F_{12}$ as a function of separation between the two cantilevers. Obviously the noise force amplitude due to fluid coupling decreases with increasing separation between the two cantilevers. This suggests the use of cantilevers with longer tips in single molecule experiments to reduce the thermal force noise amplitude. In addition to thermal noise, it is noted that the use of two (sharp) tips in CFS compared with one tip acting against a plate in one-cantilever AFM spectroscopy greatly reduces the van der Waals force in CFS. This is significant because the van der Waals forces cause adhesion of two solids and sets a background force that is not of interest in single molecule studies. 


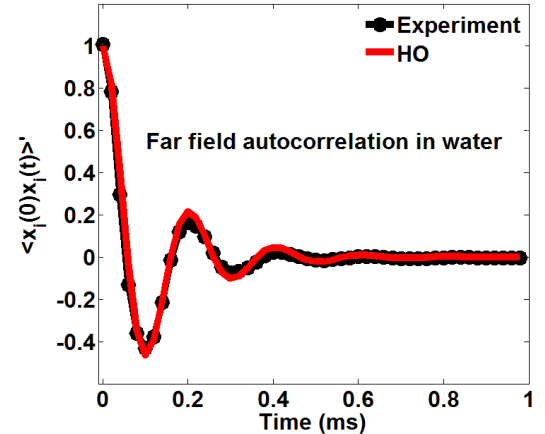

(a)

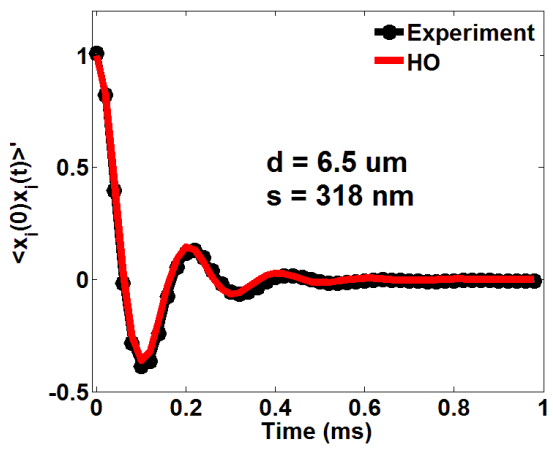

(b)

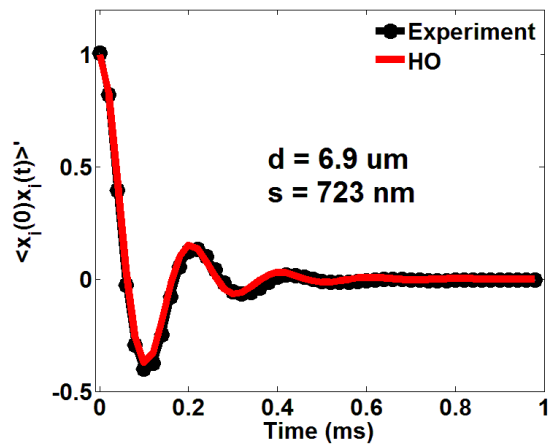

(d)

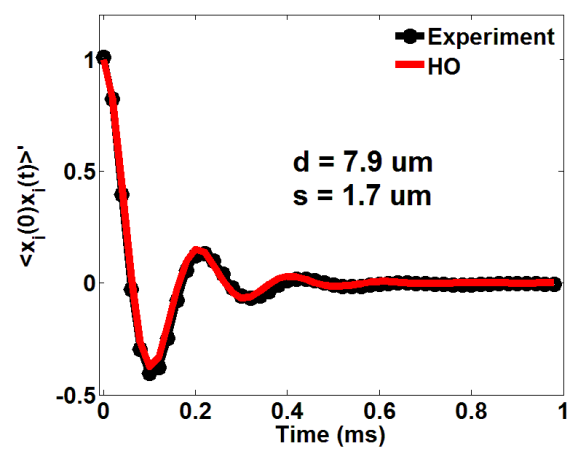

(f)

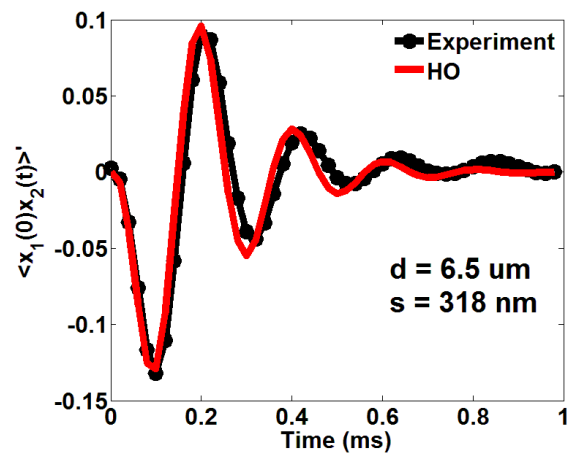

(c)

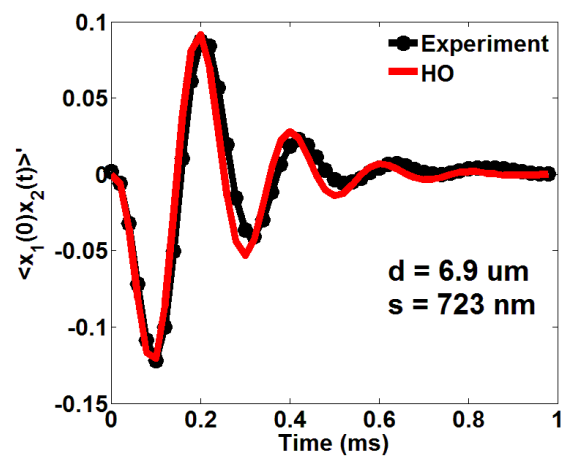

(e)

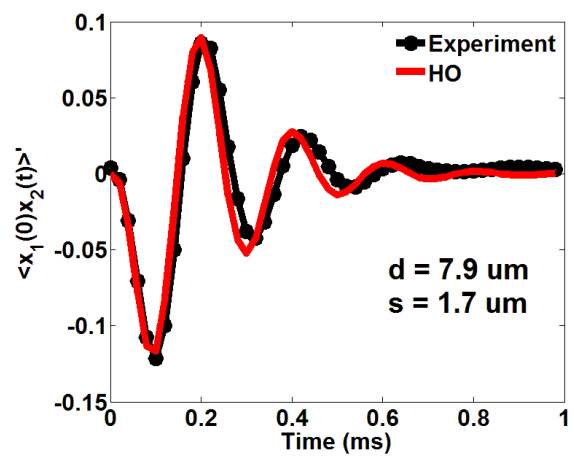

(g) 
Figure 6-4: Auto- and cross-correlation of equilibrium fluctuations in cantilever displacement for a pair of AFM cantilevers in water at $23^{\circ} \mathrm{C}$. (a) is autocorrelation at large separation, (b), (d) and (f) are autocorrelations for a series of separations, (c), (e) and (g) are cross-correlations for a series of separations. Commercial AFM cantilevers are used (ORC8 B: length $=200 \mu \mathrm{m}$, width $=$ $40 \mu \mathrm{m}, \mathrm{k}=0.1 \mathrm{Nm}^{-1}$ ). Experiment is the data measured by CFS, and HO model were calculated using the fluctuation-dissipation theory to model fluctuations via cantilever ringdown and a coupled harmonic oscillator $(\mathrm{HO})$ model for the ringdown. The prime on the left axis indicates normalized correlation function.

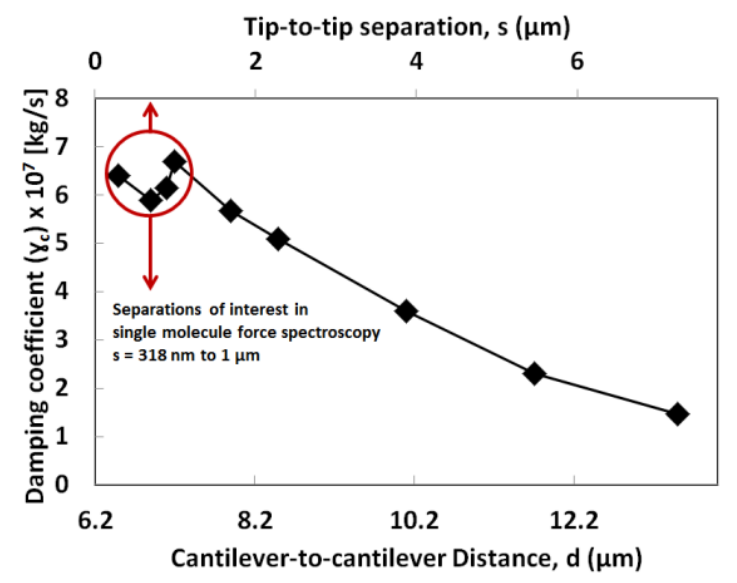

(a)

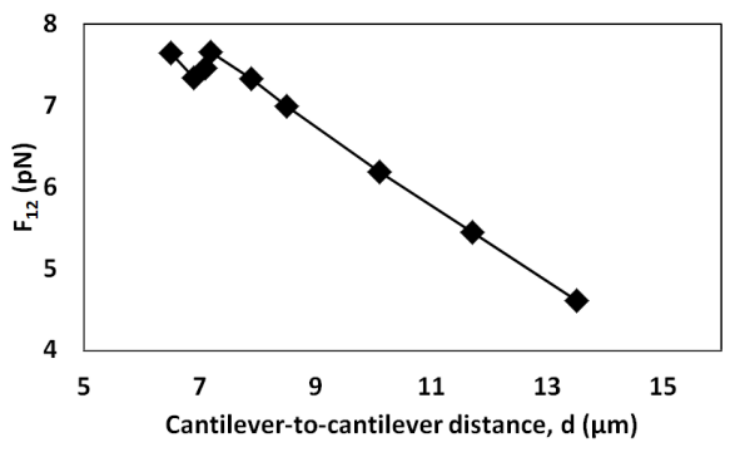

(b)

Figure 6-5: (a) Fitted damping coefficient $\gamma_{\mathrm{c}}$ as a function of separation between the cantilevers. (b) The force noise amplitude due to fluid coupling $\left(F_{12}\right)$ versus distance between the two cantilevers.

\section{Summary}

An instrument that measures cross-correlation in thermal vibrations of two closely spaced cantilevers in a tip-to-tip orientation in fluid at equilibrium was developed. The instrument is easy to incorporate into a commercial AFM and retains the full functionality of the AFM, including imaging and control of cantilever displacement. The vertically offset CFS has a lower noise floor than single cantilever measurements, and the noise force amplitude is already only 8 $\mathrm{pN}$ in the current set-up (with $0.1 \mathrm{~N} / \mathrm{m}$ stiff cantilevers that are $40 \mu \mathrm{m}$ wide and $200 \mu \mathrm{m}$ long), which is approximately $1 / 3$ of the noise in the one-cantilever spectroscopy using the same cantilever and fluid. The decreased noise arises because the noise along the length of each cantilever is not correlated; only the noise arising from fluid motion in the gap between the 
cantilevers is correlated. The correlations in thermal fluctuations of two cantilevers can be described quantitatively using a simple harmonic oscillator model. 


\section{Single Molecule Force Spectroscopy of Dextran}

The overall objective of this section of the thesis is to develop a method to measure the mechanical properties of single molecules immersed in fluid. Work in the previous chapters showed that mechanical system consisting of the two cantilevers in fluid (no straddling molecule) could be understood and modeled, which enables the study of single molecule. This chapter describes a study of the extension and fluctuations of a dextran molecule in water. Dextran was chosen because it has been the subject of a number of previous single-molecule studies (e.g. Ref. [36]), so there are known properties, and a known method for straddling the molecule between an AFM tip and a solid. The method is to coat the solid with a layer of dextran, then to touch this layer with an AFM tip. When the two solids are in contact, some dextran molecules will bind to the AFM tip and therefore straddle between the AFM tip and the solid sample. Such molecules can be stretched when the AFM tip is pulled away from the solid.

\section{Materials and Methods}

Dextran powder (31392 SIGMA; MW = 500000) was purchased from Sigma-Aldrich and was dissolved in pure water at a concentration of $10 \%(\mathrm{w} / \mathrm{v})$. AFM cantilevers were initially cleaned with pure water, then ethanol, and again water each for 1 minute, and then plasma cleaned for 5 minutes (or UV cleaned for 2 hours). A layer of dextran was deposited on the top cantilever only by the following procedure: the cantilever was immersed in the dextran solution overnight, removed and dried in air, dipped in water multiple times to remove loosely attached dextran, and then immediately used in the experiment.

The experimental procedure was the same as explained for vertical offset experiments. However, in addition to thermal deflections of the cantilevers, linear variable differential transducer (LVDT) data of the z-piezo displacement was collected at $1 \mathrm{MHz}$ using the NI card. The LVDT voltage signal was converted to displacement units using the LVDT sensitivity factor 
equal to $1.3003(\mu \mathrm{m} / \mathrm{v})$ from the manufacturer (Asylum Research, CA, USA). The Asylum Research controller and software was used to alter the displacement of the base of the top cantilever relative to the base of the bottom cantilever, $c$ as shown in Figure 7-1, and also to maintain a constant velocity of retract using a feedback loop on the LVDT.

The scheme used to pull polymers is the normal force-elongation where the top cantilever was pressed against the bottom cantilever (or a flat surface in case of single cantilever experiment), dwelled for a few seconds at a few nano-Newtons, and then retracted from the bottom cantilever (or the flat surface) at a constant velocity (10 to $50 \mathrm{~nm} / \mathrm{sec}$ ).

\section{Results}

\section{Stretching a Dextran Molecule}

The Asylum Research controller and the Igor Pro software were used to bring the top, dextrancoated cantilever into contact with the bottom, initially clean cantilever. The two were left in contact ("dwelled") for a few seconds at a compressive force of a few $\mathrm{nN}$. The base of the top cantilever was then moved away from the base of the bottom cantilever ("retracted") at a constant velocity while the NI-card recorded the deflection and LVDT signals. The LVDT measures the change in separation between the tips (or bases) of the two cantilevers (i.e. $\Delta \mathrm{LVDT}=\Delta s)$ as shown Figure 7-1. 


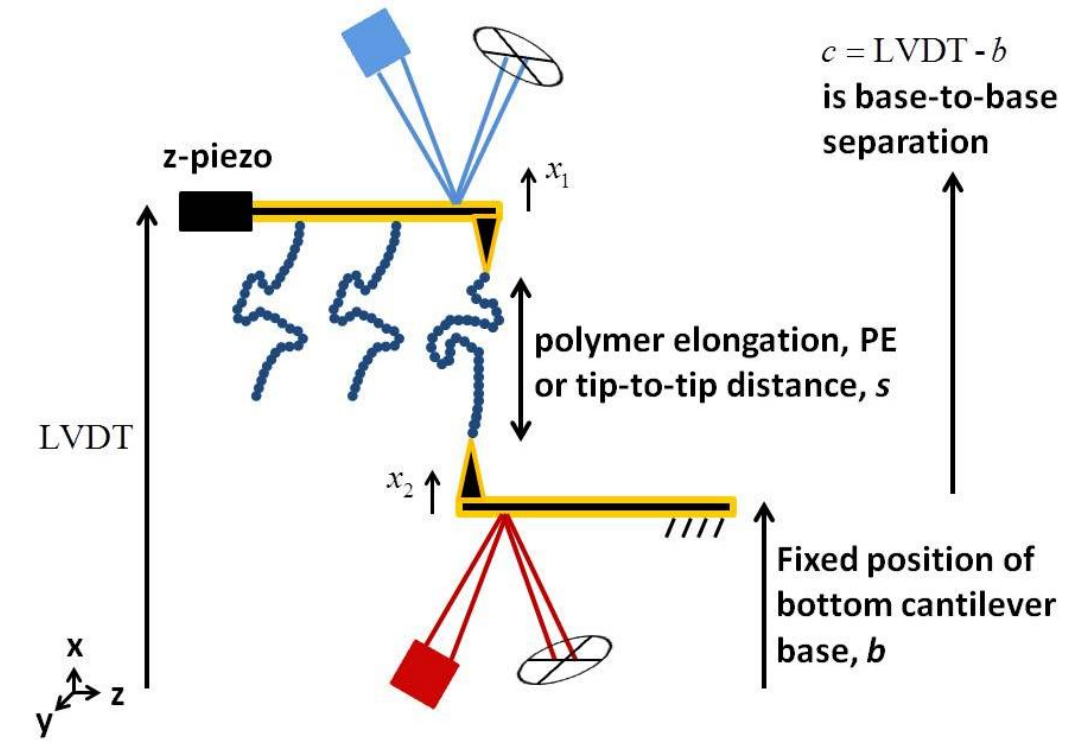

Figure 7-1: Schematic presentation of LVDT and deflection signals.

Figure 7-2-a and $\mathrm{b}$ show the deflection of the top and bottom cantilevers as a function of time during the retraction, respectively. Time zero is arbitrary, but occurs when the cantilevers are each loaded with a force pointing away from the other cantilever, i.e. an upward deflection of the top cantilever and downward deflection of the bottom cantilever. Because the velocity of the base of the top cantilever is constant, the time axis in Figure 7-2 also represents the displacement $c$ between the cantilevers bases or separation $s$ after between the cantilevers tips when they are separated (see Figure 7-1). As $c$ increases the force on each cantilever decreases in magnitude. When the two cantilevers are connected, they each experience the same magnitude of force but the directions are opposite, so parts (a) and (b) of Figure 7-2 should be mirror images. In practice, the deflection of the bottom cantilever is much noisier because of the lower quality of our electronics compared to the commercial electronics. ${ }^{5}$ In the region from $\mathrm{t}=0$ to 1.5 seconds, the deflection varies linearly. This occurs because of (a) constant retract velocity of the z-piezo, and (b) the high gradient of the contact force comparing to the cantilever stiffness. The high gradient of force is attributed to physical contact between the two tips and is usually referred to as "contact". At about $t=0.5 \mathrm{~s}$, the load switches from compressive to tensile. At $t=1.5$ to about 3 seconds, a bundle of polymers are pulled and then detached from either surfaces. At $t=$ 3 to 4 there is only one remaining polymer chain straddling between the two AFM tips and this

\footnotetext{
${ }^{5}$ The quality of electronics is improved in later version of vertically offset CFS.
} 
polymer chain is under a tensile load. At about $t=5 \mathrm{~s}$, the polymer detaches from one of the tips, causing a drop in the magnitude of force on both cantilevers, and from that time there is no longer a net average force on either cantilever. This region can be used to calibrate the properties of the fluid.

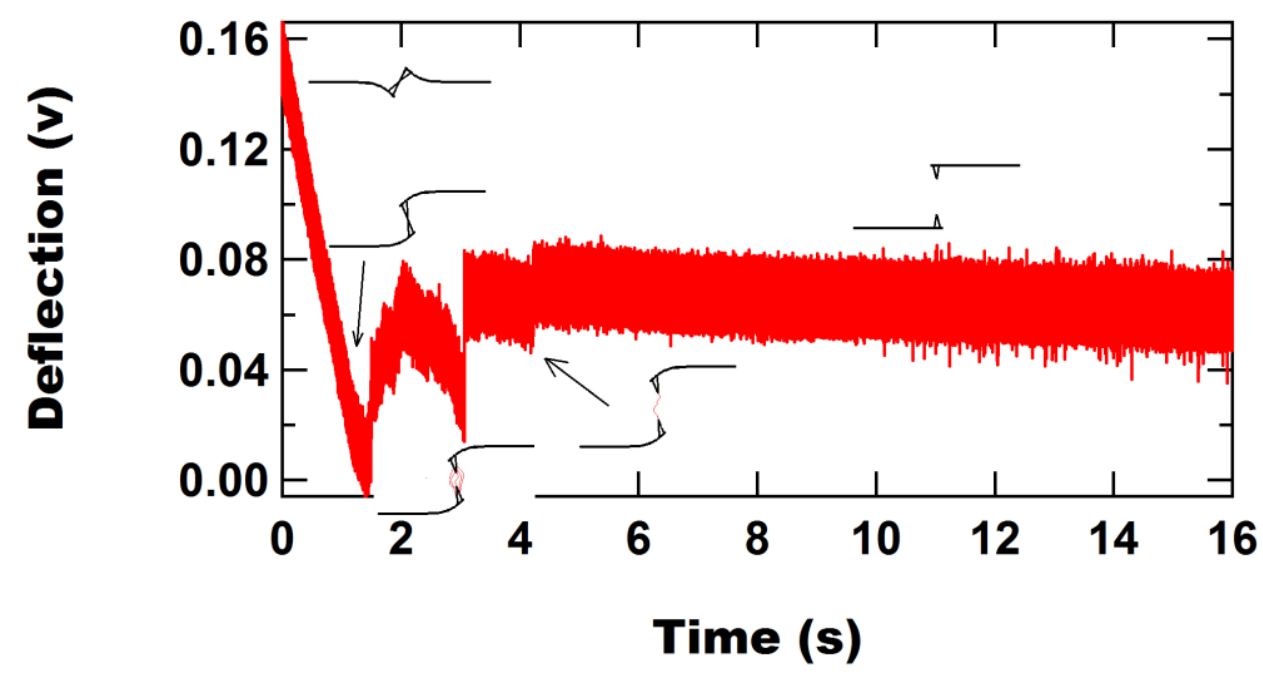

(a)

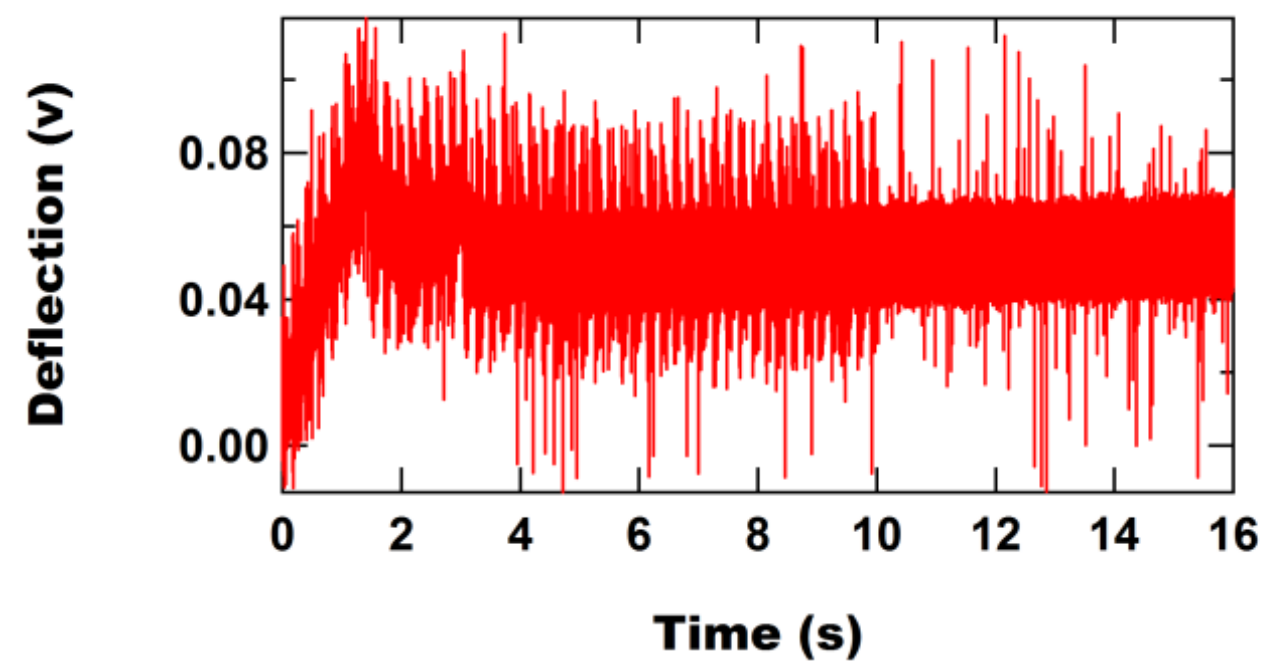

(b)

Figure 7-2: (a) Top cantilever, (b) bottom cantilever deflection signals. Bottom cantilever has a noisier background. The zero of deflection voltage is arbitrary. 
There are two ways of analyzing data: (1) static mode (similar to the conventional forceelongation experiments) which consists of measurement of the slope of force-polymer elongation curve to get molecular stiffness, (2) measuring the correlation in thermal fluctuations of the two cantilevers, i.e. correlated fluctuations (CF) mode which is a dynamic mode that gives molecular stiffness and friction.

\section{Static Force-Elongation Mode}

Initially to better resolve extension of the polymer a low pass filter is applied to the deflection signals of the top and bottom cantilevers as well as LVDT signal. Here I used a cut off frequency at $500 \mathrm{~Hz}$. This results in the following static force-elongation graphs for the top and bottom cantilevers versus LVDT as shown in Figure 7-3. With the removal of electronic noise and part of the thermal noise, it is now more clear that the two signals are equal and opposite.

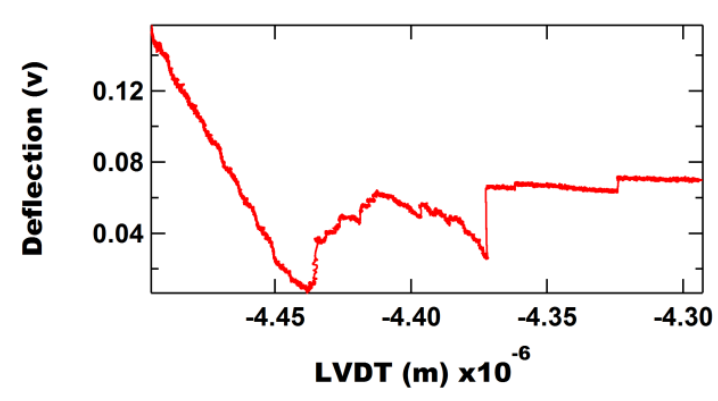

(a)

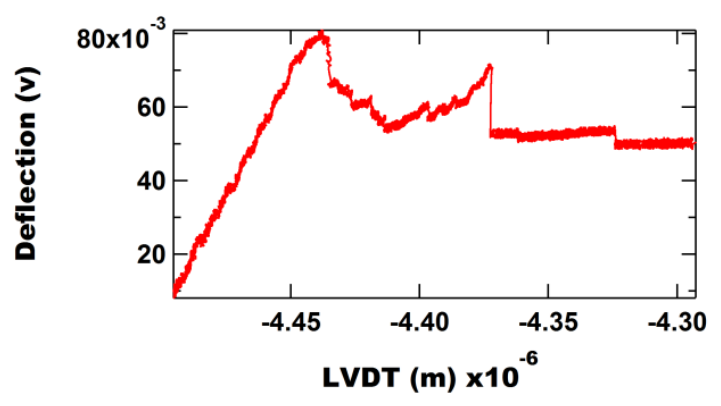

(b)

Figure 7-3: (a) Top cantilever, (b) bottom cantilever deflection signals filtered at $500 \mathrm{~Hz}$. x-axis is LVDT signal also filtered at $500 \mathrm{~Hz}$ and converted to units (m) using LVDT sensitivity factor.

Since OLS may change in the course of experiment, I correct for any possible changes by recalculating it at each pulling experiment using the portion of data where the two cantilevers are far and no polymers are attached, e.g. the last 5 seconds of recorded unfiltered deflection signals. This is done by fitting PSD of each cantilever to a simple harmonic oscillator model using the previously determined value of the spring constants: 


$$
G_{11,22}(\omega)=(\text { Ins. OLS })^{2} \times\left[\left(\frac{4 k_{B} T \gamma_{\mathrm{a}}}{\left(m(2 \pi \omega)^{2}-k_{\mathrm{c}}\right)^{2}+\left(\gamma_{\mathrm{a}}\right)^{2}(2 \pi \omega)^{2}}\right)^{1 / 2}+C\right]^{2}
$$

This equation is equivalent to equation (2.1). The OLS is used to convert the deflection signal into distance units $(m)$, then the deflection offset is removed. Results are shown in Figure 7-4 for three separate pulling experiments. In this figure, the sign of the bottom of the cantilever deflection is reversed so that the magnitudes of deflection of the top and bottom cantilevers can be compared. This comparison shows that the top and bottom cantilevers are measuring the same magnitude of forces at each position in the time-averaged ( $2 \mathrm{~ms})$ signal, as expected for a system in mechanical equilibrium. In the next section, I discuss the high frequency portion of the deflection signal, where the anticorrelated motion is used to determine the physical properties of the straddling polymer chain. 

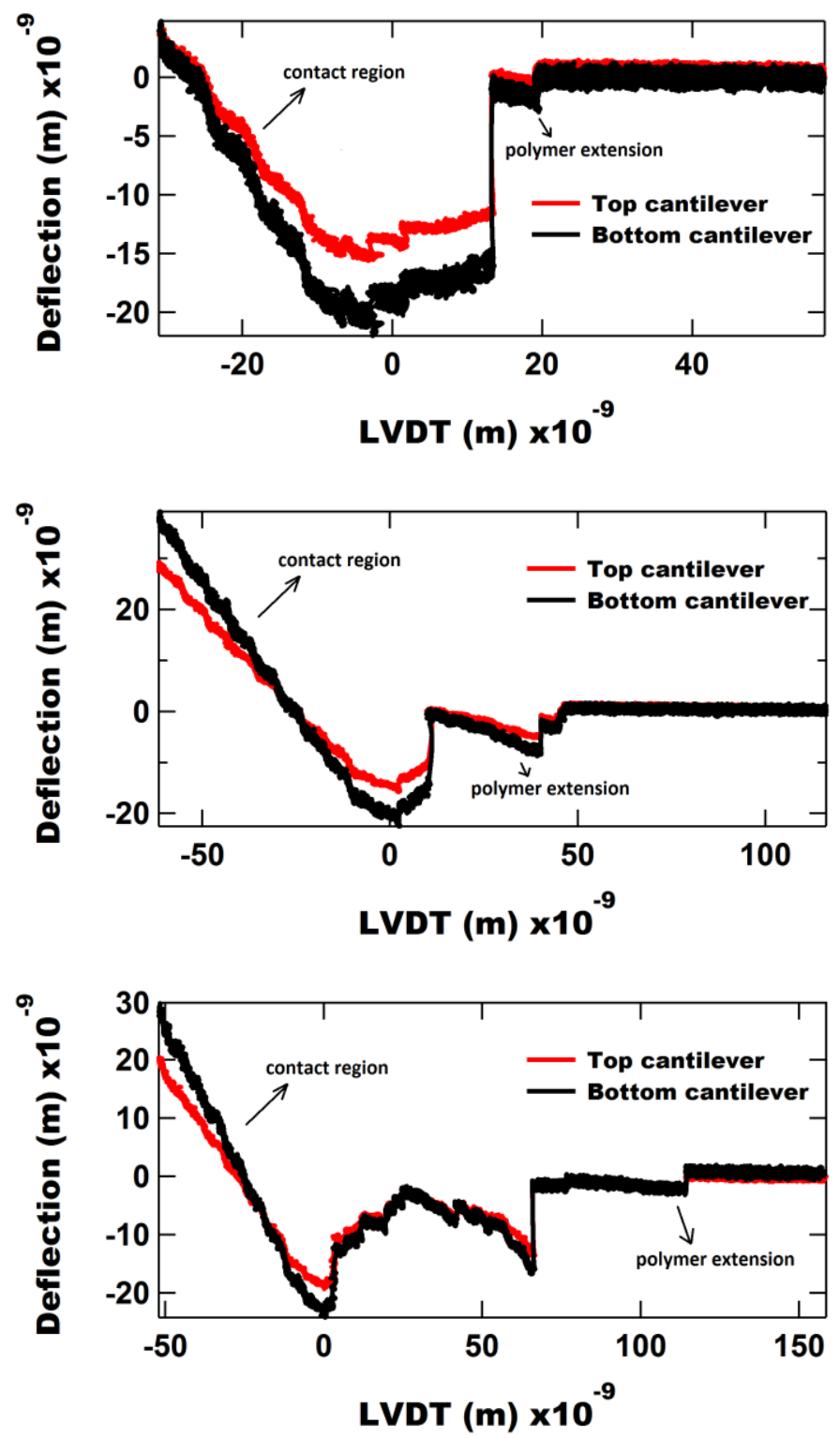

Figure 7-4: Deflection of top and bottom cantilever as a function of LVDT in dextran experiments.

Polymer extension can be calculated from equation (1.11):

$$
P E=\Delta y+\Delta x_{1}-\Delta x_{2} .
$$

The force applied on the polymer can be calculated from the top cantilever deflection:

$$
F=k_{\mathrm{c}, \mathrm{t}} \Delta x_{1} \text {. }
$$

Having PE and $F$, we can set up force-polymer extension curve as shown in Figure 7-5. 

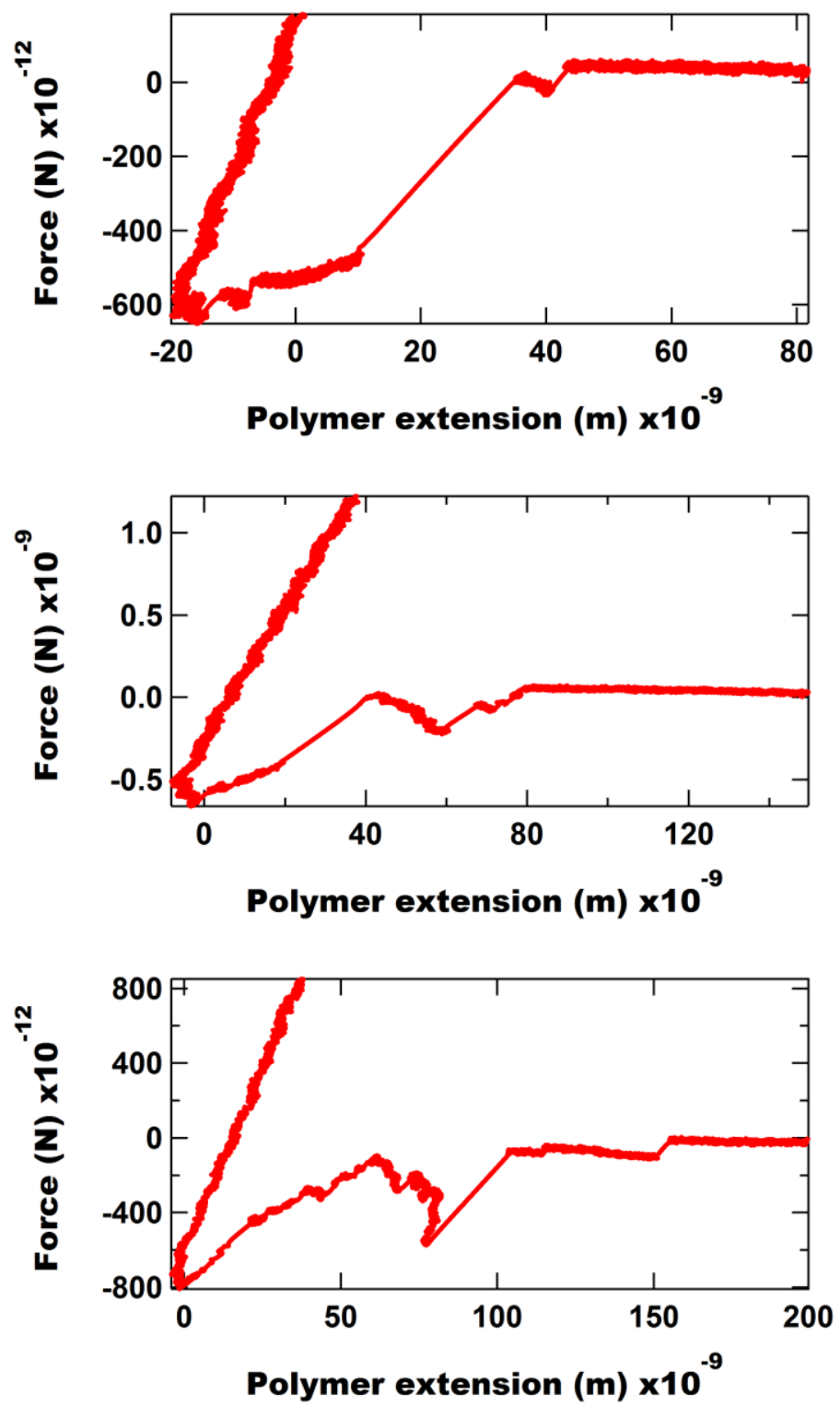

Figure 7-5: Force-polymer extension curve of dextran molecule. The rupture of the final dextran bond to the tip is at about 40, 80, and $150 \mathrm{~nm}$ (from top to bottom).

The slope of the linear region in force-polymer extension curve before polymer's detachment from one of surfaces gives average polymer stiffness at that region, i.e. static stiffness, as shown by equation (1.12):

$$
k_{p}=\frac{\delta F}{\delta P E}=\frac{\delta F}{\delta(\Delta y)-\delta F\left(1 / k_{\mathrm{c}, \mathrm{t}}+1 / k_{\mathrm{c}, \mathrm{b}}\right)} .
$$


The same region is used in the dynamic correlated fluctuations (CF) mode for comparisons of the two modes.

\section{Dynamic Correlated Fluctuations Mode}

I now perform a correlation analysis on the cantilevers connected by the spanning molecule in water as was described in the previous section with water-only. There are two additional parameters in the $\mathrm{HO}$ modeling: friction of the polymer, $\zeta_{\mathrm{p}}$, and polymer stiffness, $k_{\mathrm{p}}$. The simple harmonic oscillator model is applied to each noise spectrum, the equations of which are:

$$
\begin{aligned}
& G_{11,22, \text { no polymer }}(\omega)=2 k_{B} T\left[\begin{array}{l}
\left(\frac{\gamma_{\mathrm{a}}}{\left(m(2 \pi \omega)^{2}-k\right)^{2}+\left(\gamma_{\mathrm{a}}\right)^{2}(2 \pi \omega)^{2}}\right)+ \\
\left(\frac{\left(\gamma_{\mathrm{a}}+2 \gamma_{\mathrm{c}, \mathrm{fl}}\right)}{\left(m(2 \pi \omega)^{2}-k\right)^{2}+\left(\gamma_{\mathrm{a}}+2 \gamma_{\mathrm{c}, \mathrm{fl}}\right)^{2}(2 \pi \omega)^{2}}\right)
\end{array}\right], \\
& G_{12, \text { no polymer }}(\omega)=2 k_{B} T\left[\begin{array}{l}
\left(\frac{\gamma_{\mathrm{a}}}{\left(m(2 \pi \omega)^{2}-k\right)^{2}+\left(\gamma_{\mathrm{a}}\right)^{2}(2 \pi \omega)^{2}}\right)- \\
\left(\frac{\left(\gamma_{\mathrm{a}}+2 \gamma_{\mathrm{c}, \mathrm{fl}}\right)}{\left(m(2 \pi \omega)^{2}-k\right)^{2}+\left(\gamma_{\mathrm{a}}+2 \gamma_{\mathrm{c}, \mathrm{fl}}\right)^{2}(2 \pi \omega)^{2}}\right)
\end{array}\right], \\
& G_{11,22, \text { polymer }}(\omega)=2 k_{B} T\left(\frac{k+2 k_{\mathrm{p}}}{k+k_{\mathrm{p}}}\right)\left[\begin{array}{l}
\left(\frac{\gamma_{\mathrm{a}}}{\left(m(2 \pi \omega)^{2}-k\right)^{2}+\left(\gamma_{\mathrm{a}}\right)^{2}(2 \pi \omega)^{2}}\right)+ \\
\left.\left(\frac{\left(\gamma_{\mathrm{a}}+2 \gamma_{\mathrm{c}, \mathrm{fl}}+2 \zeta_{\mathrm{p}}\right)}{\left(m(2 \pi \omega)^{2}-k-2 k_{\mathrm{p}}\right)^{2}+\left(\gamma_{\mathrm{a}}+2 \gamma_{\mathrm{c}, \mathrm{fl}}+2 \zeta_{\mathrm{p}}\right)^{2}(2 \pi \omega)^{2}}\right)\right],
\end{array}\right]
\end{aligned}
$$




$$
G_{12, \text { polymer }}(\omega)=2 k_{B} T\left(\frac{k+2 k_{\mathrm{p}}}{k+k_{\mathrm{p}}}\right)\left[\begin{array}{l}
\left(\frac{\gamma_{\mathrm{a}}}{\left(m(2 \pi \omega)^{2}-k\right)^{2}+\left(\gamma_{\mathrm{a}}\right)^{2}(2 \pi \omega)^{2}}\right)- \\
\left.\left(\frac{\left(\gamma_{\mathrm{a}}+2 \gamma_{\mathrm{c}, \mathrm{fl}}+2 \zeta_{\mathrm{p}}\right)}{\left(m(2 \pi \omega)^{2}-k-2 k_{\mathrm{p}}\right)^{2}+\left(\gamma_{\mathrm{a}}+2 \gamma_{\mathrm{c}, \mathrm{fl}}+2 \zeta_{\mathrm{p}}\right)^{2}(2 \pi \omega)^{2}}\right)\right] .
\end{array}\right] .
$$

White noise is subtracted from $G_{11,22 \text {, no polymer }}$ and $G_{11,22 \text {, polymer }}$ using equation (7.1). In order to reduce the number of unknowns, new functions are introduced that represent the symmetric and asymmetric modes of vibration:

$$
\begin{gathered}
G_{12, s \text { no polymer }}(\omega)=G_{i j, \text { no polymer }}(\omega)+G_{i i, \text { no polymer }}(\omega)=\frac{4 k_{B} T \gamma_{\mathrm{a}}}{\left(m(2 \pi \omega)^{2}-k\right)^{2}+\left(\gamma_{\mathrm{a}}\right)^{2}(2 \pi \omega)^{2}}, \quad \text { (7.9) } \\
G_{12, a s \text { no polymer }}(\omega)=G_{i j \text {, no polymer }}(\omega)-G_{i i, \text { no polymer }}(\omega)=\frac{-4 k_{B} T\left(\gamma_{\mathrm{a}}+2 \gamma_{\mathrm{c}, \mathrm{fl}}\right)}{\left(m(2 \pi \omega)^{2}-k\right)^{2}+\left(\gamma_{\mathrm{a}}+2 \gamma_{\mathrm{c}, \mathrm{fl}}\right)^{2}(2 \pi \omega)^{2}},(7.10) \\
G_{12, s \text { polymer }}(\omega)=G_{i j, \text { polymer }}(\omega)+G_{i i, \text { polymer }}(\omega)=\frac{4 k_{B} T \gamma_{\mathrm{a}}\left(\frac{k+2 k_{\mathrm{p}}}{k+k_{\mathrm{p}}}\right)}{\left(m(2 \pi \omega)^{2}-k\right)^{2}+\left(\gamma_{\mathrm{a}}\right)^{2}(2 \pi \omega)^{2}}, \quad(7.11) \\
G_{12, a s \text { polymer }}(\omega)=G_{i j, \text { polymer }}(\omega)-G_{i i, \text { polymer }}(\omega)=-\frac{4 k_{B} T\left(\gamma_{\mathrm{a}}+2 \gamma_{\mathrm{c}, \mathrm{fl}}+2 \zeta_{\mathrm{p}}\right)\left(\frac{k+2 k_{\mathrm{p}}}{k+k_{\mathrm{p}}}\right)}{\left(m(2 \pi \omega)^{2}-k-2 k_{\mathrm{p}}\right)^{2}+\left(\gamma_{\mathrm{a}}+2 \gamma_{\mathrm{c}, \mathrm{fl}}+2 \zeta_{\mathrm{p}}\right)^{2}(2 \pi \omega)^{2}} .
\end{gathered}
$$

where the subscript $s$ denotes, symmetric mode, and as denotes asymmetric mode (shown schematically in Figure 6-3). The important point is that $\gamma_{\mathrm{c}, \mathrm{fl}}$ due to fluid coupling the two cantilevers does not appear in equations (7.9) and (7.11): i.e. the symmetric motion is unaffected by the fluid coupling and all the fluid coupling is contained in the antisymmetric mode. In experiment, equivalent functions are obtained as: $G_{12, s}=G_{12}+\left(G_{11}+G_{22}\right) / 2$ and $G_{12, a s}=G_{12}-\left(G_{11}+G_{22}\right) / 2$. The fits to $G_{12, s}$ and $G_{12, a s}$ are done simultaneously with a single set of parameters. 
When there is no polymer attached between the two cantilevers, parameters of the system such as $k_{\mathrm{c}}, m, \gamma_{\mathrm{a}}$ and $\gamma_{\mathrm{c}, \mathrm{fl}}$ are obtained from fits to equations (7.9) and (7.10). Averaged spring constant, fluid loaded mass and damping coefficients were calculated from these data over a range of separations (from a few tens of nanometer to hundreds of nanometers) and used to calculate polymer stiffness and friction coefficient, from fits to equations (7.11) and (7.12). Sample fits of equations (7.9) to (7.12) to experimental data are shown in Figure 7-6:

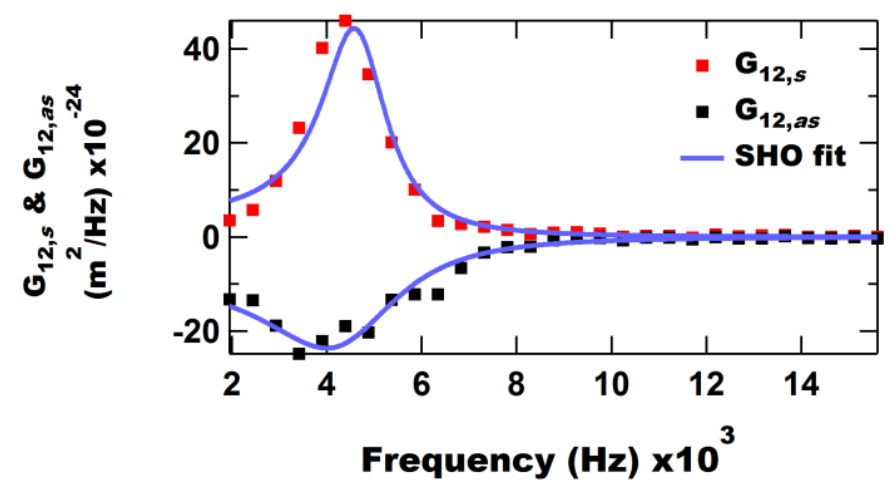

(a)

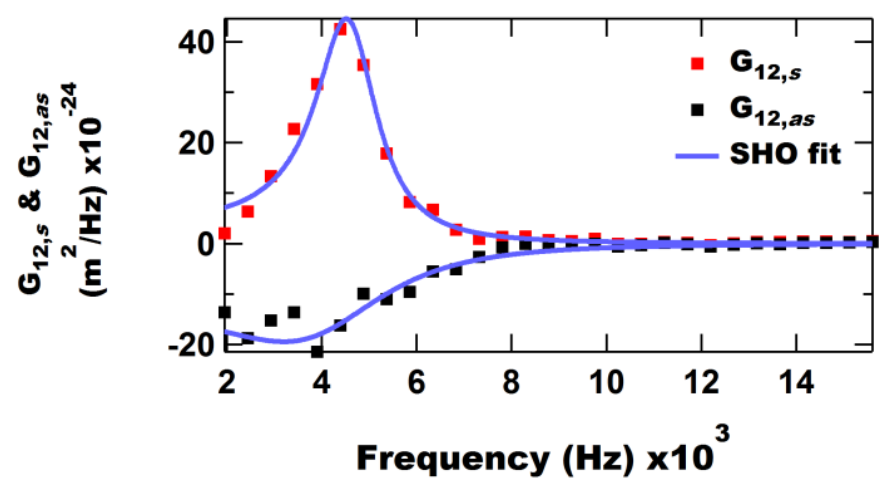

(b)

Figure 7-6: Values of $G_{12, s}$ and $G_{12, a s}$ obtained from experiment (symbols) for (a) $578 \mathrm{~nm}$ tip-totip separations (without polymer) and (b) $152 \mathrm{~nm}$ tip-to-tip separation (straddling polymer). Fits to equations (7.9) to (7.12) (lines) are also shown, using $m, k, \gamma_{\mathrm{a}}, \gamma_{\mathrm{c}, \mathrm{fl}}, k_{\mathrm{p}}$ and $\zeta_{\mathrm{p}}$. A different fit is used for the experiment (a) and (b).

Figure 7-7-a shows the measured polymer stiffness as a function of force. Results are shown for both static force-elongation mode, F-mode (fluctuations mode that is a dynamic mode using the 
single cantilever AFM, see Ref. [36]) and dynamic CF-mode using the same data. Figure 7-7-b shows the measured polymer friction coefficient as a function of force. Results are shown for Fmode [36] and dynamic CF-mode using the same data. These results show that the polymer stiffness and friction increases with force. It is useful to validate these data against existing data for dextran. I used the data and method (i.e. F-mode) from Ref. [36] and plotted on the same graph for comparison. The measurements of Kawakami et al. are for greater extension force, but my measurements using CFS have a similar magnitude. The data also show that the dextran molecule has a stiffness of about $0.015 \mathrm{~N} / \mathrm{m}$ and a friction of about $2 \times 10^{-7} \mathrm{~kg} / \mathrm{s}$ when subject to a force up to $200 \mathrm{pN}$.

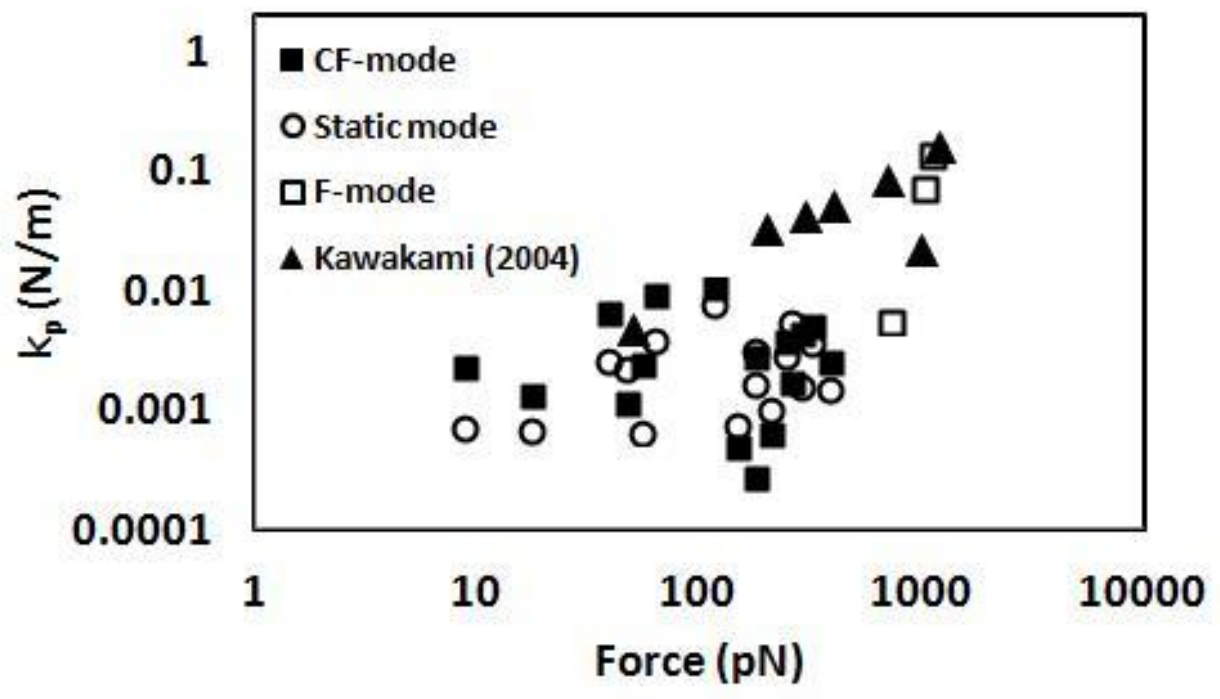

(a) 


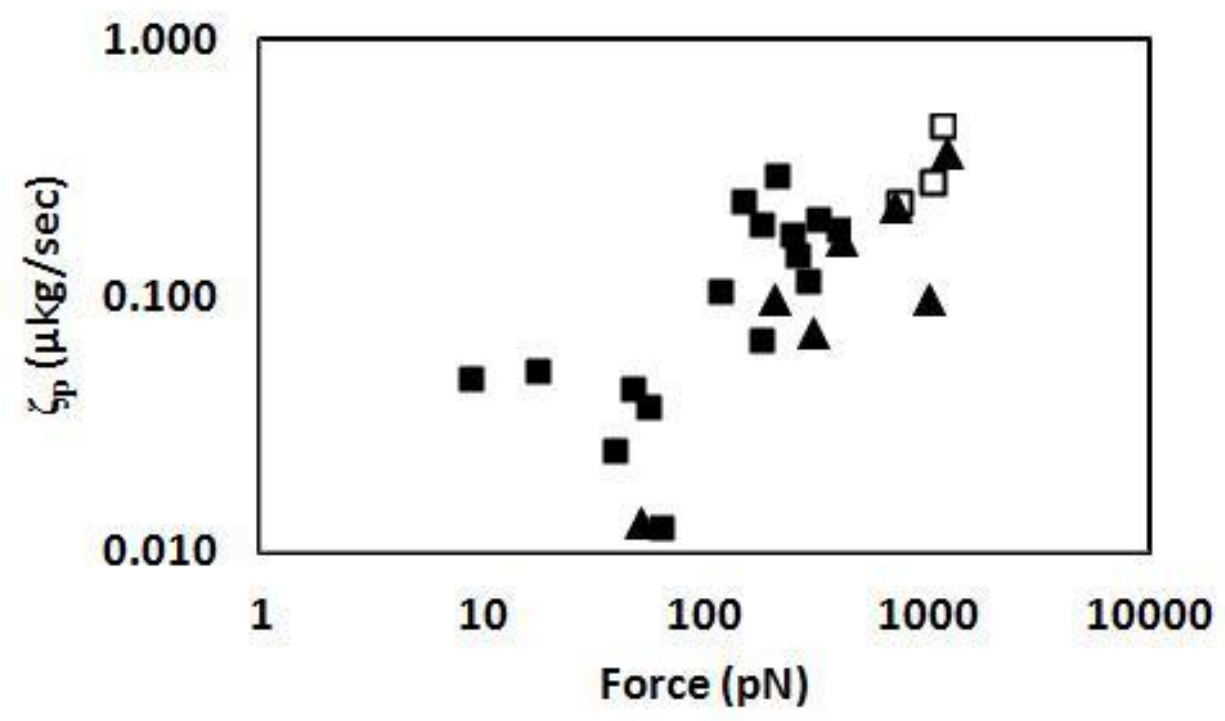

(b)

Figure 7-7: (a) Stiffness of dextran obtained from dynamic correlated fluctuations (CF) mode, static force-elongation mode, fluctuations $(\mathrm{F})$ mode of a single cantilever and compared with results of Kawakami, et al. [36] as a function of force. (b) Friction of dextran obtained from dynamic correlated fluctuations (CF) mode, and fluctuations (F) mode of a single cantilever and compared with results of Kawakami, et al. [36] as a function of force.

\section{Discussion}

Comparing static-mode to CF-mode or F-mode there are significant differences between results from the low frequency method to the high frequency methods. It is not clear yet whether the methods are measuring different things, or there is an error in one of them. The static-mode measures low frequency stiffness of the polymer (less than $500 \mathrm{~Hz}$ ), CF-mode or F-mode measure high frequency stiffness of the polymer (about $5 \mathrm{kHz}$ and $20 \mathrm{kHz}$ respectively). It is not expected that the polymer exhibits different stiffness at these stimulation frequencies. Nevertheless, it is noted that Kawakami, et al. [36] found similar stiffness for dextran using a related static and dynamic method (fluctuations of a single cantilever, i.e. F-mode) at similar frequencies to this work. This suggests that there is an error in our measurement or analysis. Errors can arise due to lack of frequency resolution in the dynamic CF-mode (currently $=488$ 
$\mathrm{Hz}$ ) and errors in OLS measurements which manifest itself in the non-vertical contact region in Figure 7-5.

I associate the scattering of dextran data to: (a) polydispersity of dextran resulting in different contour length of the pulled molecules, (b) more specifically the non-specific adhesion of the molecules on the surface which results in (i) slipping of the molecules on surface, and that (ii) bundles of dextran molecules are entangled on the surface, and that (iii) pulled chains are anchored on one side to other chains exhibiting complex elasticity and friction. All these shortcomings need to be addressed by improving the vertically-offset CFS experimental methods, choice of molecule, choice of immobilizing the molecule on surface and surface preparation. These issues motivated the experiments on selectively bound, monodisperse DNA in the next chapter.

\section{Summary}

This chapter shows1 that CFS is able to measure polymer stiffness and friction. The correlations in thermal fluctuations of two cantilevers with a tethered molecule can be described quantitatively using a simple harmonic oscillator model, and provide two parameters: the polymer stiffness and friction coefficient. The friction measurements provide a new parameter to characterize polymers. The polymer stiffness measurements have been measured before, but mainly near zero frequency. The mismatch between zero frequency measurements and high frequency measurements needs further investigations. 


\section{Single Molecule Force Spectroscopy of Single-Stranded DNA}

\section{Rationale}

Due to experimental uncertainties in methods used in experiments with dextran in the previous chapter, I decided to use monodisperse molecules with specific chemical attachment to each AFM tip. The obvious choice of monodisperse molecule is DNA, and gold-thiol chemistry was used to anchor the DNA to a single AFM tip. The free end of each DNA strand contained a complementary section such that DNA bound to the top cantilever would hydrogen bond to the DNA on the bottom cantilever to produce a molecule that spanned between the two AFM tips. Major improvements were also made to the previous version of the vertically offset CFS. For example, experiments in this chapter are performed in closed fluid cell rather than an open droplet environment. To add to knowledge currently available on DNA, I decided to study single stranded DNA that is less studied than double-stranded DNA.

\section{Introduction}

ssDNA is an essential intermediate in many DNA metabolic processes such as replication, recombination, repair, and transcription, and it is specifically recognized by many DNA-binding proteins, and thus its behavior is of growing interest to the scientific community [124-133]. Some of the biological actions of ssDNA depend on its mechanical properties [51], which has motivated studies of the mechanical properties of ssDNA by single molecule force spectroscopy (SMFS) [134]. Two trends are obvious in the literature: $(i)$ most studies have been on long ssDNA $(n>100$, where $n$ is the number of nucleotides) [12] whereas short $\operatorname{ssDNA}(n<100)$ is encountered in nature during replication and in important technologies such as recombinant DNA technology [2], (ii) most experiments have examined homopolymeric ssDNA [51, 134136] whereas most natural DNA is of necessity heteropolymeric to carry the genetic code. Thus, the current work addresses the important area of short, heteropolymeric ssDNA. Most previous 
work on heteropolymeric ssDNA utilized spectroscopic techniques such as Förster resonance energy transfer (FRET) or scattering techniques small angle X-ray scattering (SAXS) [51, 133, $134,136]$.

In this chapter, I investigate the mechanical properties of ssDNA using CFS. Traditional AFM experiments produce a load-extension curve, which can be compared with model polymer properties. For example, the Worm-like Chain (WLC) model of polymer elasticity has been successful in describing the properties of nucleic acids and polypeptides [50-52]. The force required to extend a polymer chain in the WLC model was given as equation (3.1) [49] and is reproduced here:

$$
F \cong f\left[-2\left(1-\frac{\langle R\rangle}{R_{\max }}\right)+\frac{1}{2}\left(1-\frac{\langle R\rangle}{R_{\max }}\right)^{-2}+\frac{3}{2}\right] .
$$

From equation (8.1), the stiffness of a WLC, $k_{\mathrm{wLC}}(=d F / d\langle R\rangle)$, is (equation (3.3)):

$$
\frac{R_{\max } k_{\mathrm{wLC}}}{f}=H^{-1}\left(\frac{F}{f}\right)
$$

where $H^{-1}$ is the inverse function $H$ (equation (3.4)):

$$
H(x) \cong-2(x-2)^{-1 / 3}+\frac{1}{2}(x-2)^{2 / 3}+\frac{3}{2} .
$$

Equations (8.1), and (8.2) and (8.3), or another equation expressing WLC end-to-end distance probability distribution (see for example Ref. [53]), are used to calculate the persistence length $l_{\mathrm{p}}=k_{\mathrm{B}} T / f$. Recent SMFS attempts have been to measure $l_{\mathrm{p}}$ as a function of salt concentration [51, 135], valence [133], and number of nucleotides [135, 137]. Other recent attempts to measure $l_{\mathrm{p}}$ of ssDNA include transient electric birefringence (TEB) [135], AFM imaging [138], and Monte Carlo simulation [139, 140].

While equation (8.1) is commonly used to interpret mechanical SMFS, it can only be used to measure molecular elasticity, whereas the behavior of a molecule is much more complex. There are frequency-dependent responses to the displacement (stiffness), velocity (friction), and acceleration (mass) of various parts of the molecule as well as the center of mass. The dynamics 
of these molecules has significant effect on the biological performance of molecules. For example, the high values of the friction coefficient of a molecule enhances its resistance to folding [61, 66, 72, 134, 141-143]. The friction coefficient is the force divided by the velocity. Most mechanical SMFS measurements are static, that is they cannot give any information on molecule dynamics, but recently dynamic mechanical SMFS has been used to quantify both the stiffness and hydrodynamics of single chains [36, 39, 71, 141, 142]. An interesting, yet not fully understood, feature of these measurements is the very high values of the fiction coefficient. This friction coefficient is usually considered in two parts: the motion of the entire molecule through the solvent (center of mass motion) which is described in terms of a Stokes drag, and internal friction of the molecule due to relative motion of different parts of the molecule. An exciting aspect of CFS is that it directly monitors the end to end distance of a polymer molecule, and the two components of friction are easily separated: the symmetric motion monitors the center of mass friction and the antisymmetric motion monitors the internal friction. During polymer folding, different parts of the molecule could be moving at different velocities, but the CFS directly monitors only changes in the end-to-end distance so the internal friction coefficient is expressed in terms of the end-to-end velocity. Clearly the end-to-end friction depends on the detailed friction of various parts of the molecule, but these are not resolved.

Dissipation due to segments of the molecule moving through the solvent can be modeled using the Rouse model [49]. It was suggested that at low frequency and low stretch $(F \ll f)$ this is the dominant form of dissipation for sufficiently long polymers [67]. However, at high force ( $F \gg f$ ), the internal friction becomes the dominant source of dissipation [40, 71, 141]. In this force regime, the measured values of friction coefficient are orders of magnitude larger than the predictions of Rouse model [71]. For WLC at high force the stiffness and friction are given by [50] (this is the high force estimate of equations (8.2) and (8.3) that are also shown in equation (3.14)):

$$
\begin{gathered}
k_{\mathrm{WLC}}=\frac{\sqrt{8}}{R_{\max } \sqrt{f}} F^{3 / 2}, \\
\zeta_{\mathrm{WLC}}=\frac{\zeta_{\mathrm{B}}}{8 l_{p}^{1 / 2} R_{\max }\left(k_{\mathrm{B}} T\right)^{3 / 2}} F^{3 / 2},
\end{gathered}
$$


where $\zeta_{\mathrm{B}}$ is the bending internal friction coefficient in units of $\mathrm{kgm}^{3} \mathrm{~s}^{-1}$ that resists folding. These equations were given in Chapter 3 as equations (3.14) and (3.48). Equations (8.4) and (8.5) predict that both the stiffness and internal friction of a WLC increase as $F^{3 / 2}$ at high force.

This chapter describes CFS measurements on short ssDNA [100]. No molecular handle was used and the length of ssDNA was $n=60$ nucleotide. I performed these measurements with high force resolution (less than $4 \mathrm{pN}$ ) and frequency $(2 \mathrm{kHz})$. The high force resolution is achieved by (i) application of the softest commercially available AFM cantilevers $\left(k_{\mathrm{c}}=0.003 \mathrm{~N} / \mathrm{m}\right)$ and (ii) correlation measurement [144]. Application of soft cantilevers reduces the thermal noise force to about $4 \mathrm{pN}$. This is about one fifth of the force required to break 10 to $20 \mathrm{G}-\mathrm{C}$ or A-T base pairs in a dsDNA [32]. Application of correlation measurement reduces the noise force limit to about $1 / 3$ of noise inherent in one biolever [144]. The high frequency measurement is achieved by performing correlation measurements at the fundamental resonance frequency of the two biolevers. The fundamental resonance frequency of these biolevers in solvent was about 2 $\mathrm{kHz}$. This localizes our measurement at this frequency.

Equations (8.2) to (8.4), and the known persistence length, $l_{\mathrm{p}}$, were used to model the stiffness of ssDNA and were compared to the measured data. I use equation (8.5) to model the internal friction of ssDNA. The effective value of bending internal friction coefficient $\zeta_{\mathrm{B}}$ was obtained by fitting equation (8.5) to the experimental data. I believe that this is the first measurement of internal friction coefficient of short ssDNA. 


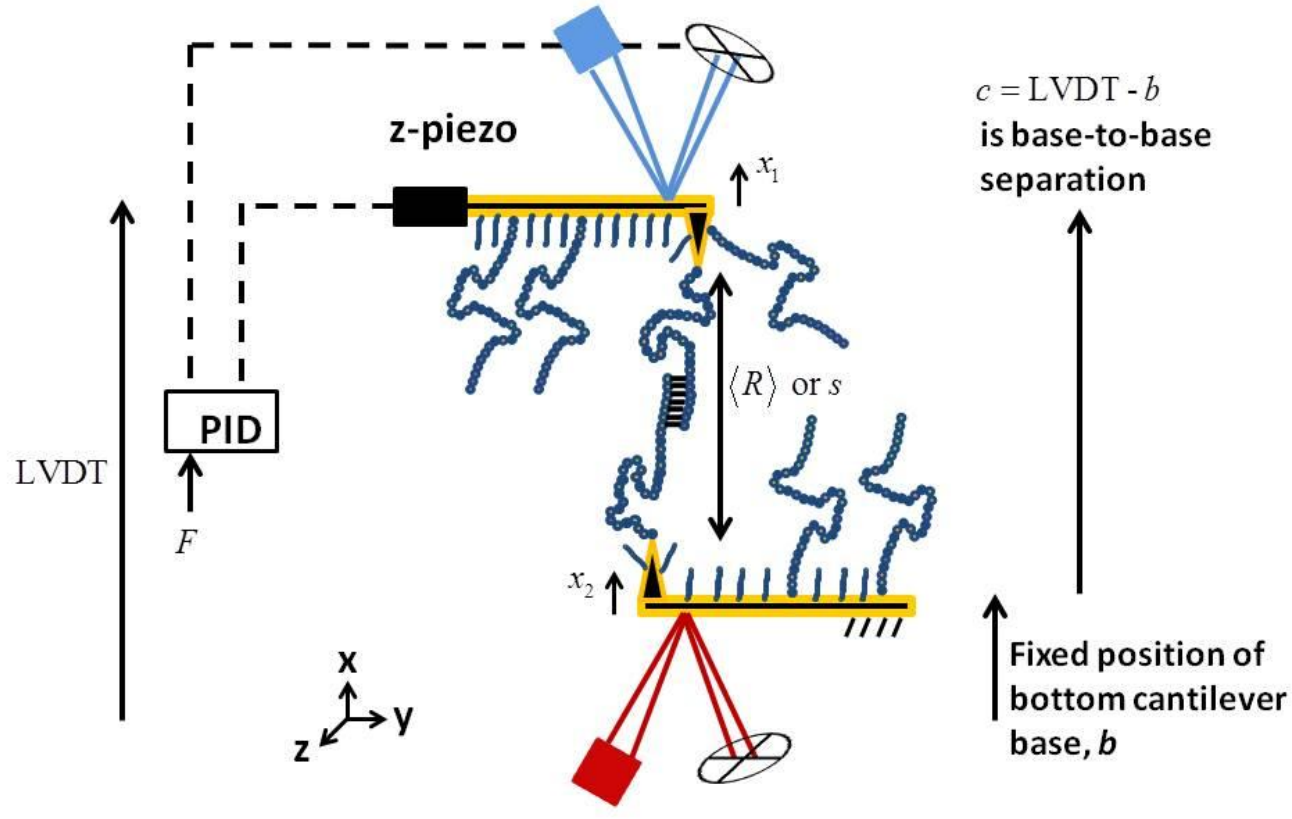

Figure 8-1: Schematic of CFS experiment. $\langle R\rangle$ is the average molecule elongation. $x_{1}$ and $x_{2}$ are the thermal fluctuations in displacement of the cantilevers that are detected by individual detection systems. A z-piezo drive mechanism is used to vary $c$ and thus to alter the elongation of the molecule. A PID feedback loop is used to maintain a constant force $F$ on the molecule during the force clamp period. The schematic shows a molecule of ssDNA on each tip bound to each other in a short base-pairing section.

\section{Materials and Methods}

Figure 8-1 is a schematic of the experiment in CFS. Two cantilevers ("biolevers", gold coated on tip side, BL-RC-150VB, Asylum Research, Oxford Instruments, Santa Barbara, CA) are placed in close proximity of each other while the their vertical separation is varied using a $\mathrm{z}^{-}$ piezoelectric drive mechanism attached to the top cantilever. The vertical displacement of the $\mathrm{z}$ piezo is denoted with LVDT. A slow PID feedback loop is used on static deflection of the top cantilever to maintain a clamp force $F$ on the molecule while the thermal fluctuations of each cantilever, $x_{1}$, and $x_{2}$, are measured. The top detection system is part of a commercial AFM (MFP3D-Bio, Asylum Research, Oxford Instruments) that includes a superluminescent laser with a frequency of $860 \mathrm{~nm}$. The customized bottom detection system includes a laser 
(51nanoFCM, Schäfter + Kirchhoff $\mathrm{GmbH}$ ), with a frequency of $680 \mathrm{~nm}$, and a photosensitive diode (QP50-6-18u-SD2, Pacific Silicon Sensor). The bottom detection system is mounted underneath the AFM microscope plate (Nikon, TE2000U). Thus the bottom cantilever and detection system essentially replace "the sample" in a normal experiment. The stiffness and resonance frequency of cantilevers were calibrated using the thermal or Hutter method [121] in solvent prior to experiment and are $0.003 \mathrm{~N} / \mathrm{m}$ and $2 \mathrm{kHz}$ respectively when the tips are macroscopically separated from any sample.

The static AFM measurements (schematic not shown) are the normal force-elongation experiments, see for example [32]. In this case a cantilever is used against a solid surface. To gold coat the glass surface, a thin layer of adhesive chromium, $57 \AA$, and then a thin layer of gold, $60 \AA$, were evaporated using E-beam evaporator. All surfaces were cleaned with UVgenerated ozone, ethanol and water.

The two types of ssDNA were purchased from Biosearch Technologies (Petaluma, CA) [32]. Type A:

5'-SH-(ACATGCCTCCGAAGGATTATTGGTTTGAGTTTTTTTTTTTTTTTTTTTTT)-3'

Type B:

5'-(AAAAAAAAAAAAAAAAAAATGAGTTTGGTTATTAGGAAGCCTCCGTACA)-SH-3'.

A $10 \mu \mathrm{M}$ solution of ssDNA was prepared in Tris-EDTA buffer (TE, Thermo Scientific). A deprotection reaction is required prior to immobilization since the thiolated end of ssDNA (5' and 3' in type A and type B respectively) is protected with a disulfide bond to 6-mercapto hexanol $(\mathrm{MCH})$. To reduce the disulfide bond, I added $25 \mathrm{mM}$ tris(2-carboxyethyl)phosphine (TCEP, Thermo Scientific) to the solution for one to two hours. After this step, each cantilever was incubated in a different reduced ssDNA solution over night (or one cantilever was incubated in ssDNA type A solution, and solid surface was incubated in ssDNA type B solution). In some experiments a passivation step was used where a solution of $1 \mu \mathrm{M} \mathrm{MCH}$ (Sigma) was added to the incubation solution after one hour. The purpose was to fill in any vacant gold sites where the bulky ssDNA could not fit. The ssDNA strands were anchored to the cantilever tip (or the surface) via gold-thiol reaction. The ssDNA-coated cantilevers (or one ssDNA-coated cantilever 
and a ssDNA-coated glass) were then put in a closed fluid cell (Bioheater, Asylum Research, Oxford Instruments). Type A and type B have 20 complementary bases ( $\mathrm{T}$ and A respectively) at their nonthiolated end. Upon approach of the biolevers, up to 20 complementary bases conjugate due to A-T base paring. Upon retract of the cantilevers, the dsDNA region can hold a few pico-Newtons of force for a period of time before breaking. All the measurements were performed in phosphate buffer saline buffer (PBS, pH 7.4, Sigma) and at room temperature ( $\left.22^{\circ} \mathrm{C}\right)$.

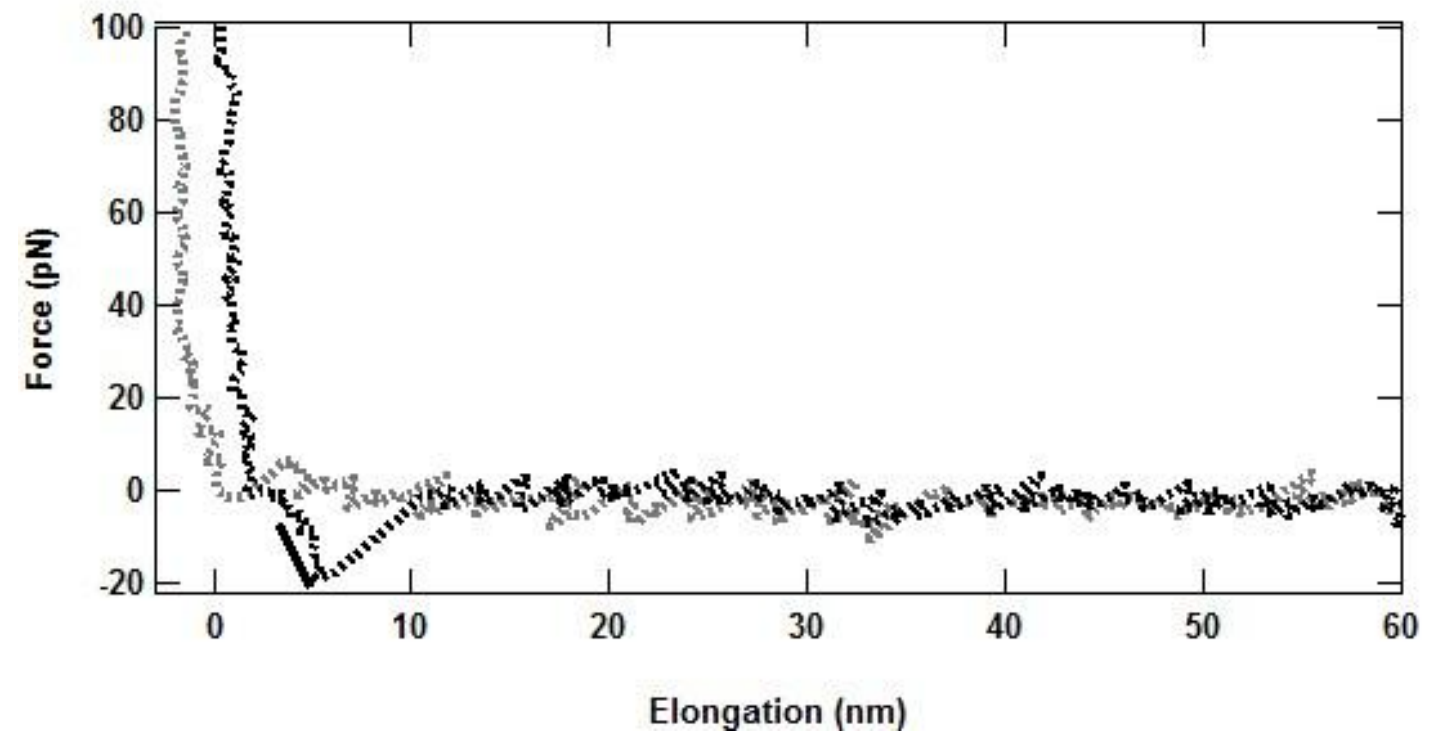

Figure 8-2: Force-elongation of ssDNA obtained using AFM, and low pass-filtered ( $\mathrm{f}=500 \mathrm{~Hz}$ ) to show quasi-static force. Both approach (grey) and retract (black) curves are shown using dotted lines. The solid line shows a linear fit to force-elongation data which results in static stiffness.

Two different measurements were performed: a slow measurement of deflections while varying the separation, and a high frequency measurement of the deflection at a constant force. The slow measurement $(\sim 1 \mathrm{kHz})$ was used to capture the static force-elongation curve of ssDNA with a typical approach/retract velocity of $100 \mathrm{~nm} / \mathrm{sec}$ to $1 \mu \mathrm{m} / \mathrm{s}$ (see Figure 8-2). This measurement is best performed using AFM (one cantilever against a solid surface) since the thermal noise is readily reduced using a low pass filter on deflection. Such a measurement cannot give any information about the dynamics of ssDNA at frequencies greater than the low-pass filter. It does not give any information about the internal friction. 


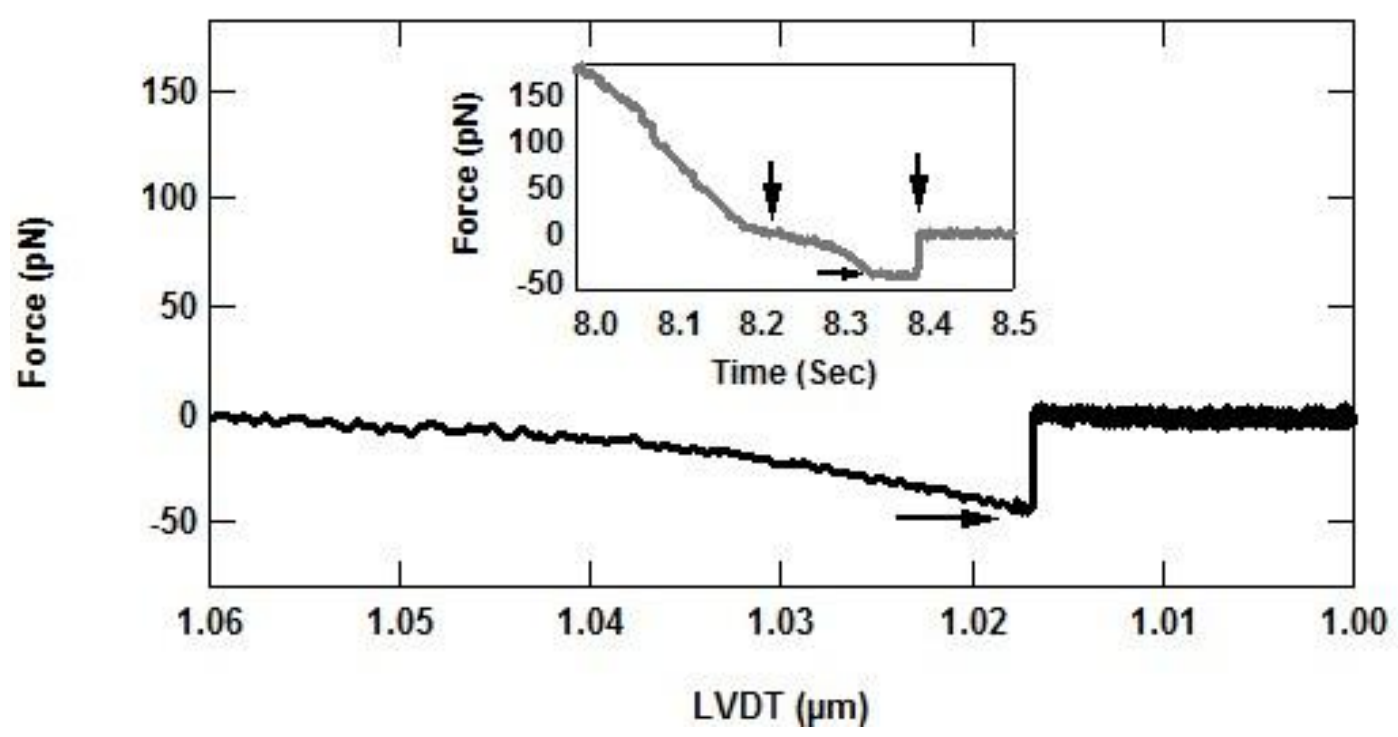

Figure 8-3: Force-LVDT curve of ssDNA pull and clamp in the dynamic force-clamp mode of CFS. In this experiment, $c$ is increased with time (as measured by the LVDT) while the force is measured. When the force reaches a preset threshold (horizontal arrow), a feedback loop is automatically activated to maintain a constant force. This force is shown by the horizontal arrow in both the main figure and the inset. The inset is the force-time curve of ssDNA during the pull and the clamp. The left vertical arrow in the inset shows the start of the force-LVDT curve in the parent figure $(\mathrm{LVDT}=1.06 \mu \mathrm{m})$. The right vertical arrow shows the time at which the dsDNA splits apart and the molecule no longer spans between the two tips. The LVDT and time values on horizontal axes are arbitrary. The start of the clamp period is labeled by the horizontal arrow in the inset.

The fast measurement $(1 \mathrm{MHz})$ is used to collect the simultaneous thermal fluctuations of the two cantilevers, which are then correlated and fitted to obtain the high-frequency mechanical properties of ssDNA. The correlations are measured in a "stationary" state, where the polymer is at a constant force. The start of this time period is labeled by the horizontal arrow in the inset for Figure 8-3. The end of the clamping period, shown by a vertical arrow at a later time, shows the time when the hydrogen bonds between the two DNA-DNA molecules broke. In biology this is referred to as "melting". The two time series of fluctuations were collected using a high frequency data acquisition card (NI PCI-6110, National Instrument, Irvine, CA). 


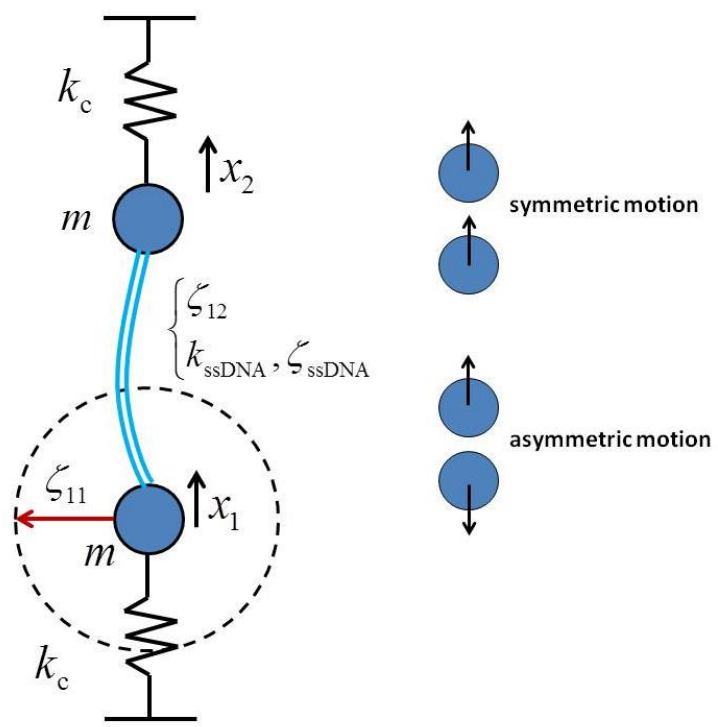

Figure 8-4: Schematic of the analysis. Each cantilever is modeled as a simple harmonic oscillator with mass $m$, stiffness $k_{\mathrm{c}}$, and damping $\zeta_{11} \cdot \zeta_{12}$ is the hydrodynamic fluid coupling between the cantilevers. $k_{\mathrm{ssDNA}}$ and $\zeta_{\mathrm{ssDNA}}$ are the stiffness and friction coefficient of the DNA straddling between the two cantilevers. The directions of the symmetric and asymmetric modes are shown.

\section{Modal Analysis}

The thermal fluctuations of the two cantilevers, $x_{1}$ and $x_{2}$, were measured $(i)$ while the chain was clamped at force $F$ (filled circle in Figure 8-3) and (ii) in the post-melt region. The time series of the sum in fluctuations $\left(X_{s}=\frac{1}{\sqrt{2}}\left(x_{1}+x_{2}\right)\right)$ was used to calculate the power spectral density of symmetric mode and the time series of the difference in fluctuations $\left(X_{a s}=\frac{1}{\sqrt{2}}\left(x_{1}-x_{2}\right)\right)$ was used to calculate the power spectral density of the asymmetric mode (see Figure 8-4). Recall that I checked that the time-lag between the two detection systems was insignificant. These coordinates are orthogonal and independent, i.e. $\left\langle X_{s}(t) X_{a s}(0)\right\rangle=0$. I used Langevin theoretical framework to quantify the dynamics of the system:

$$
\left(\begin{array}{c}
m \ddot{x}_{1} \\
m \ddot{x}_{2}
\end{array}\right)+\left(\begin{array}{cc}
\zeta_{11}+\zeta_{\mathrm{ssDNA}} & \zeta_{12}-\zeta_{\mathrm{sSDNA}} \\
\zeta_{21}-\zeta_{\mathrm{ssDNA}} & \zeta_{22}+\zeta_{\mathrm{ssDNA}}
\end{array}\right)\left(\begin{array}{c}
\dot{x}_{1} \\
\dot{x}_{2}
\end{array}\right)+\left(\begin{array}{cc}
k_{\mathrm{c}}+k_{\mathrm{ssDNA}} & -k_{\mathrm{ssDNA}} \\
-k_{\mathrm{ssDNA}} & k_{\mathrm{c}}+k_{\mathrm{ssDNA}}
\end{array}\right)\left(\begin{array}{l}
x_{1} \\
x_{2}
\end{array}\right)=\left(\begin{array}{l}
f_{n 1}(t) \\
f_{n 2}(t)
\end{array}\right),
$$


where $m$ is the mass of the cantilever, and $\overline{\bar{\zeta}}$ is the friction tensor: $\zeta_{11}$ is the friction coefficient of a single cantilever motion and $\zeta_{12}$ is the friction coefficient of mutual motion of the two cantilevers without straddling DNA. $k_{\mathrm{ssDNA}}$ and $\zeta_{\mathrm{ssDNA}}$ are the stiffness and friction coefficient of the straddled ssDNA chain. The friction tensor is symmetric with equal diagonal elements. $f_{n 1}(t)$ and $f_{n 2}(t)$ are the Brownian forces with two moments:

$$
\left\langle f_{n i}(t)\right\rangle=0, \quad \mathrm{i}=1,2
$$

and,

$$
\left\langle f_{n i}(t) f_{n j}\left(t^{\prime}\right)\right\rangle=2 \zeta_{\mathrm{ij}} k_{B} T \delta\left(t-t^{\prime}\right), \quad i, j=1,2
$$

where $\delta$ is the Dirac delta function. In terms of the symmetric and asymmetric coordinates, equation (8.6) forms:

$$
m \ddot{X}_{k}+\zeta_{k} \dot{X}_{k}+k_{k} X_{k}=f_{b k},
$$

where $k=s$ or as. $\zeta_{s}$ and $\zeta_{a s}$ are the friction coefficients in the symmetric and asymmetric modes; $k_{s}$ and $k_{a s}$ are the spring constants in the symmetric and asymmetric modes; and similarly, $f_{\text {ns }}$ and $f_{\text {nas }}$ are the Brownian forces sensed by the two cantilevers in the symmetric and asymmetric modes respectively. The power spectral density in each mode $s$ or as is $G_{k}=4 \int_{0}^{\infty}\left\langle X_{k}^{2}(t)\right\rangle \cos (\omega t) d t$ which from fluctuation-dissipation theorem is [43]:

$$
G_{s}=\frac{4 k_{B} T \zeta_{s}}{\left(m \omega^{2}-k_{s}\right)^{2}+\zeta_{s}^{2} \omega^{2}},
$$

and,

$$
G_{a s}=\frac{4 k_{B} T \zeta_{a s}}{\left(m \omega^{2}-k_{a s}\right)^{2}+\zeta_{a s}^{2} \omega^{2}} .
$$

Note again that the components of the friction are separated: the power density spectrum of the symmetric mode depends only on the center-of-mass friction (equation (8.10)) whereas the power density spectrum of the antisymmetric mode depends only of the "internal" friction that arises when the chain is elongated in the asymmetric mode. 
In order to obtain properties of the straddling molecule (the DNA), two regions are to be measured: the clamped region and post-melt region (see inset to Figure 8-3). The symmetriccorrelation power spectral density $G_{\mathrm{s}}$ (equation (8.10)) of the clamped region and post-melt region are expected to be the same: the stiffness and internal friction of the straddled ssDNA does not affect the symmetric motion and the ssDNA-solvent friction is too small compared to the cantilever-solvent friction to sense the changes due to melting. This is shown in Figure 8-5.

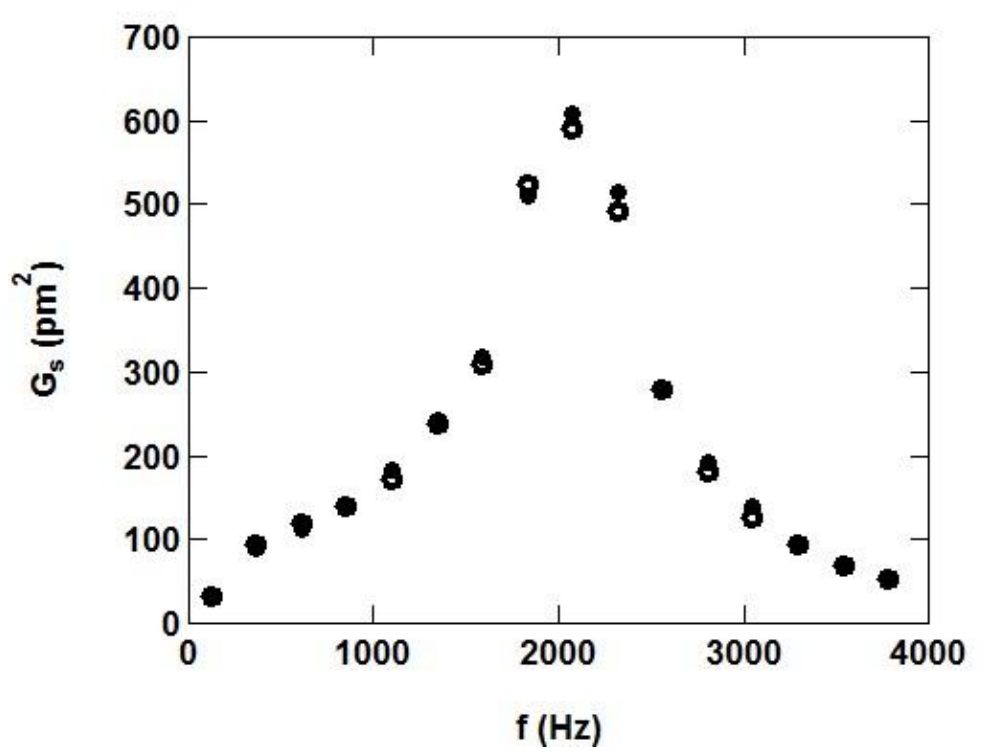

Figure 8-5: Symmetric component of the correlation power spectral density in the clamped region (full circle) and in post-melt region (open circle). Results of only one experiment is shown.

The properties of the molecule straddling between the tips are obtained from the comparison of asymmetric-correlation power spectral density $G_{\text {as }}$ (equation (8.11)) in the clamped region and post-melt regions (see Figure 8-6). The stiffness and friction coefficient (which will be shown to be the internal friction of the chain) are:

$$
k_{\mathrm{ssDNA}}=\frac{k_{\mathrm{as}, \text { clamp }}-k_{\mathrm{as}, \text { post-melt }}}{2},
$$

and,

$$
\zeta_{\mathrm{ssDNA}}=\frac{\zeta_{\mathrm{as}, \mathrm{clamp}}-\zeta_{\mathrm{as}, \text { post-melt }}}{2} .
$$


It is noted that in equation (8.6), except for $\zeta_{12}, \zeta_{\mathrm{ssDNA}}$, and $k_{\mathrm{ssDNA}}$, all the other parameters are known a priori: $m_{\mathrm{c}}=k_{\mathrm{c}} /\left(2 \pi f_{0}\right)^{2} \cong 20 \mathrm{ng}$ where $f_{0}=2 \mathrm{kHz}$ is the resonance frequency, and $\zeta_{11}=200 \mu \mathrm{g} \mathrm{s}^{-1}$ in solvent. I measure the thermal fluctuations and produce the power spectral densities of the asymmetric modes in the clamped region and the post-melt region. I then fit the experimental asymmetric-correlation power spectral density $G_{\text {as }}$ to equation (8.11) to obtain $k_{a s}$ and $\zeta_{a s}$ (see Figure 8-6). The stiffness and friction coefficient of the straddled chain are then obtained from equations (8.12) and (8.13) respectively. 


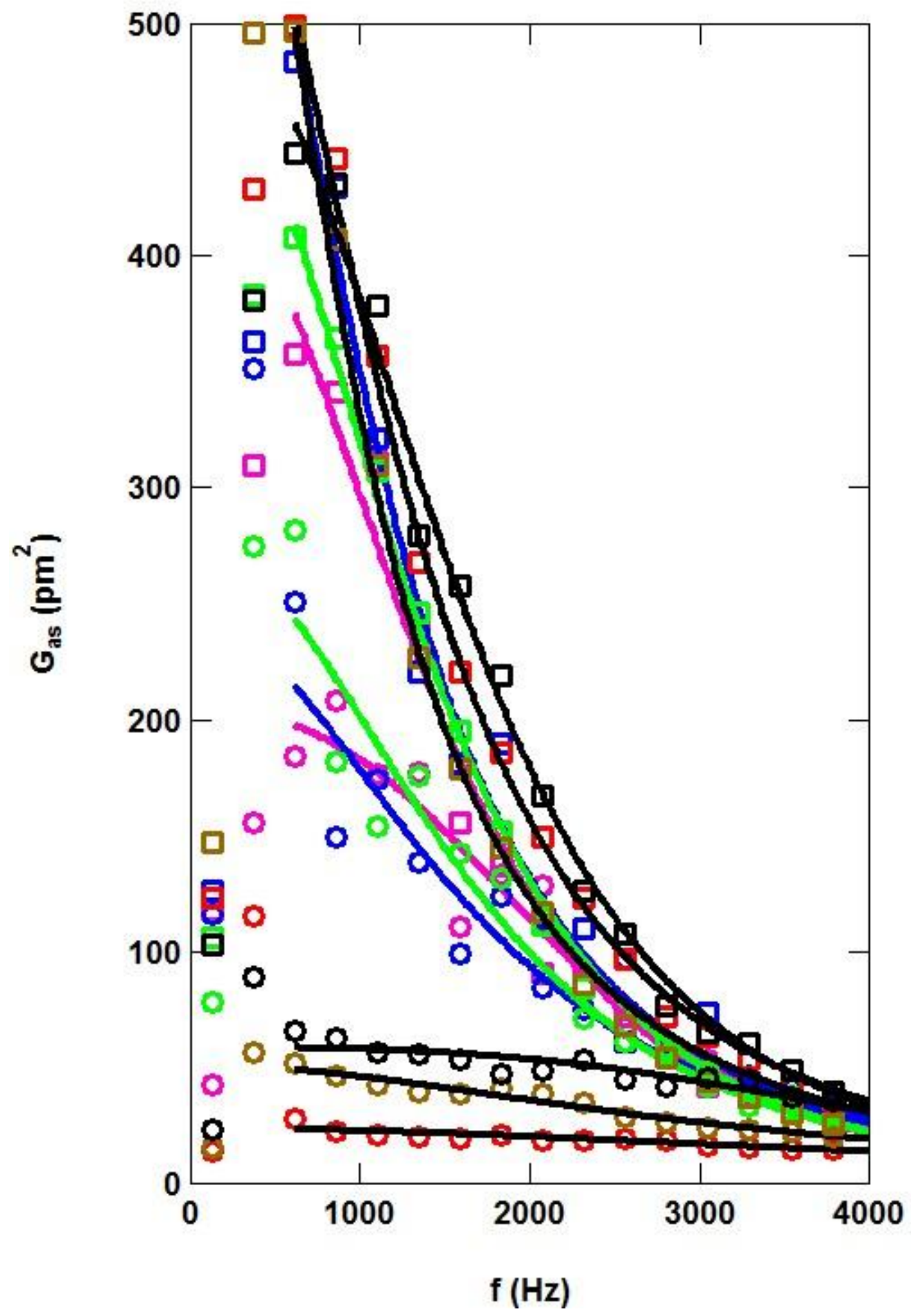

Figure 8-6: Asymmetric-correlation power spectral density in the clamped region (circle) and in post-melt region (square). Different colors show different experiments. Solid lines show the quality of fits of equation (8.11) to experiment. Three lines are drawn in color to match the same color experimental symbols. Equation (8.11) is fitted to experimental $G_{\text {as }}$ from $600 \mathrm{~Hz}$ to $4 \mathrm{kHz}$. 


\section{Results}

A single chain elongates upon application of tensile force $F$ (in a form know as the dumbbell model) [55]:

$$
\zeta \frac{d\langle R\rangle}{d t}+k\langle R\rangle=F,
$$

where $\zeta$ and $k$ are the friction coefficient and spring constant of the chain respectively, and $\langle R\rangle$ is the chain end-to-end distance. For a chain elongation rate, $d\langle R\rangle / d t$, that is much slower than the relaxation time of the chain, $\tau \equiv \zeta / k$ [55], equation (8.14) reduces to a static force balance: $k \Delta\langle R\rangle=\Delta F$, where $\Delta$ denotes variation of the quantity. In dynamic measurements where $F$ is in on the same scale as $\tau$ (either due to thermal motion [36] or external drive [39]), the unsteady term $\zeta d\langle R\rangle / d t$ becomes important. Thus while $k$ of a single molecule can be measured in a static scheme, only a dynamic scheme gives both $\zeta$ and $k$.

Static force-elongation measurement using conventional AFM reveals the molecular stiffness and the length of the polymer chain. In addition to the dynamic measurement using CFS that is explained below, I performed static force-elongation measurements of ssDNA using a conventional AFM "pulling" experiment. The purpose of the static measurement was to compare the measured stiffness in the conventional and dynamic data to validate the dynamic measurement. Previous investigation showed that the stiffness of static and dynamic measurement are in quantitative agreement [36, 39], but in the dextran measurements in the previous chapter, the two were not in agreement. Results for the two measurements are shown in (Figure 8-7) and show agreement, helping to validate the CFS measurement. The static stiffness is calculated from the slope of force-elongation curve (see thick solid line in Figure 8-2) prior to splitting of the dsDNA section (Figure 8-1). The sudden decrease in the force (or deflection) of the cantilever designates the splitting of the dsDNA section. I performed the static measurement at various z-piezo retract velocities ranging from $100 \mathrm{~nm} / \mathrm{s}$ to $1 \mu \mathrm{m} / \mathrm{s}$; however, I did not resolve 
any variation in force-elongation curves or molecular stiffness with velocity. At much larger retract velocities, however, one would expect that the frictional forces in the molecule become important ( $\zeta d\langle R\rangle / d t$ in equation (8.14)), and thus there is a limit to the retract velocity used in static AFM measurements of molecule stiffness. In addition, high-retract-velocity measurements boost solvent friction on the entire cantilever, which is a large effect that will obscure molecular events.

The other important parameter gained form static measurement was the length at which the molecule ruptures. I find that while there is a distribution of rupture lengths (standard deviation $10 \mathrm{~nm}$, data not shown), the average rupture length is $\sim 16 \mathrm{~nm}$, less than the contour length of the molecule $R_{\max }=42 \mathrm{~nm}$ expected from the number of nucleotides $(n=60)$ multiplied by the interphosphate distance in ssDNA $(\sim 0.7 \mathrm{~nm}[145])$. The rupture length smaller than the contour length is expected since the two strands of ssDNA are connected via a dsDNA section (Figure 8-1) with only 20 paired bases of $\mathrm{A}$ and $\mathrm{T}$. Well before the chain reaches its fully extended length and ruptures due to breakage of a covalent bond, the dsDNA section melts;. The expected rupture force is about $20 \mathrm{pN}$ at typical AFM retract velocities [32]. Measurement of the rupture length is less data intensive in conventional (quasistatic) AFM measurement than in dynamic CFS measurement because the conventional measurements is performed at a much lower bandwidth.

Dynamic force-clamp measurement using CFS reveals the molecular stiffness and internal friction coefficient. Results of the molecular stiffness of ssDNA obtained from dynamic CFS and static AFM are shown in Figure 8-7. Clearly the stiffness increases with the applied load, and the CFS result agrees with the conventional AFM result. The dynamic stiffness obtained from equations (8.2) -(8.4) using the published literature for the persistence length of homopolymeric ssDNA [51] of $l_{\mathrm{p}}=2.5 \mathrm{~nm}$, and the contour length of $R_{\max }=42 \mathrm{~nm}$ is also shown. There is agreement between theory and experiment, again validating the CFS technique. The error bar (bottom right corner) gives our estimate of the measurement error in each experiment. The main source of error in the force is thermal noise force $=\sqrt{k_{\mathrm{c}} k_{\mathrm{B}} T}=4 \mathrm{pN}$ from equipartition theorem. A shortcoming in our measurement is that I cannot define a standard 
deviation in stiffness data since I do not have multiple measurements on one specific single molecule at one specific force. The error estimate comes from the average deviation of the experimental points from the theoretical stiffness-force relationship (equations (8.2) and (8.3)) for $l_{\mathrm{p}}=2.5 \mathrm{~nm}$, which is $\sim 2.4 \mathrm{mN} / \mathrm{m}$.

I also made the best fit of equations (8.2)-(8.4) using known values of contour length, which gives a persistence length of $\sim 3 \mathrm{~nm}$, slightly longer than the previous measurement for homopolymeric ssDNA. This difference could be due to uncertainties in calibration of cantilever spring constant or photodiode optical lever sensitivity or in the work of others who determined the value of the persistence length in earlier measurements. The main conclusion of this section is however that short ssDNA of mixed nucleotides exhibit similar elasticity as a homopolymeric chain [51]. It is noted that this is a case where no hairpins are formed when the chain is highly stretched $F \gg 1 \mathrm{pN}$. Formation of higher-order structures due to base pairing could change this result.

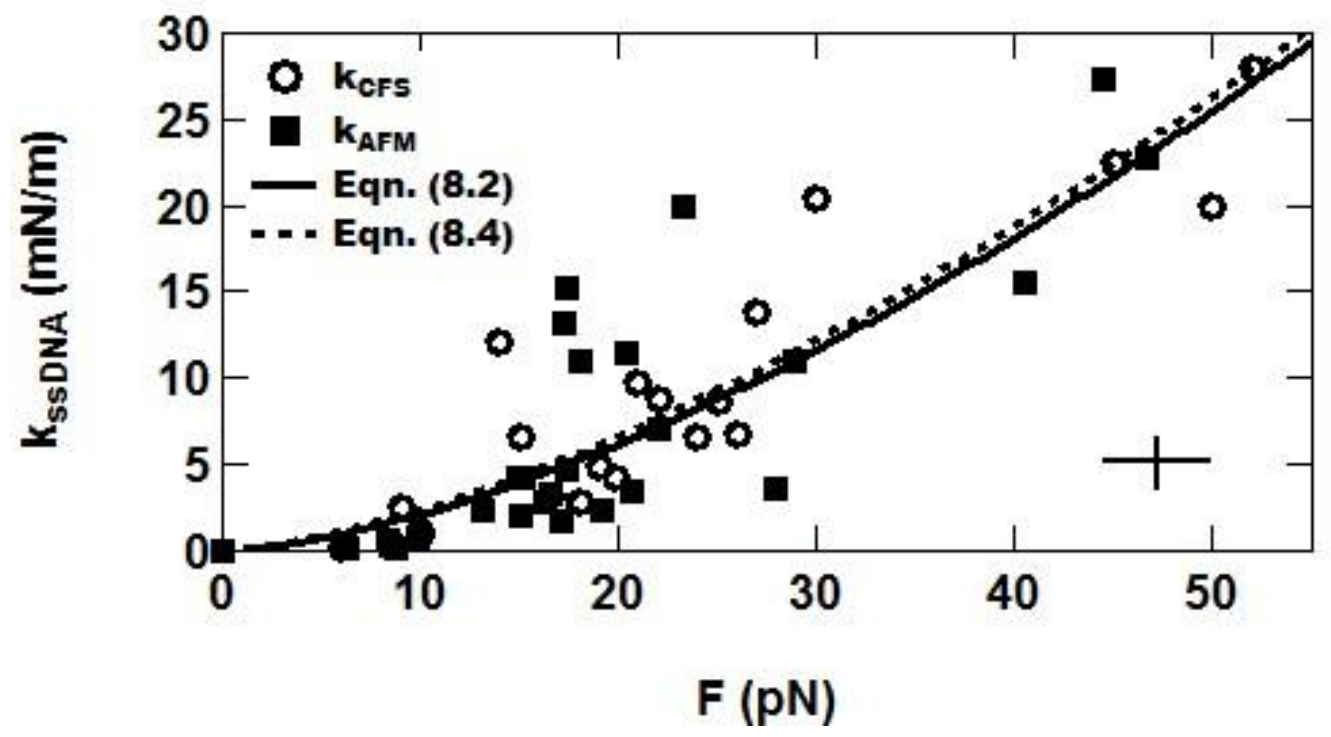

Figure 8-7: Stiffness of ssDNA measured in dynamic force-clamp mode of CFS (open circle) and static force-elongation mode of AFM (filled square). Solid line and dashed line are WLC model equation (8.2) and (8.4) respectively with $R_{\max }=42 \mathrm{~nm}$ and $l_{\mathrm{p}}=2.5 \mathrm{~nm}$. 
Figure 8-8 shows the friction coefficient of ssDNA as a function of force. The Rouse friction coefficient of 60 nucleotide long ssDNA due to solvent hydrodynamic interaction is $\zeta_{R}=N \zeta_{b} \cong 0.8 \mu g s^{-1}$, where $N=8$ is the number of Kuhn segments of length $b=2 l_{\mathrm{p}}$ and $\zeta_{b}=6 \pi \eta b \cong 0.1 \mu \mathrm{gs}^{-1}$ is the friction coefficient of one Kuhn segment [49].

Our data shows an enhanced friction coefficient, which I attribute to internal friction of the chain $[67,71]$. In the dynamic force-clamp experiment, the mean amplitude of thermal fluctuations in the asymmetric mode is $2 A \cong 2 \mathrm{~nm}$, where $A=\sqrt{k_{\mathrm{B}} T / k}$ is the root-mean-squared amplitude of thermal fluctuations of one cantilever from the equipartition theorem. This results in bending vibrations in the chain with $2 \mathrm{~nm}$ amplitude and at $2 \mathrm{kHz}$. For short ssDNA, this level of bending at high stretch ( $F \gg 1 \mathrm{pN}$ ) results in significant internal friction that dominates ssDNAsolvent friction $[50,67,71]$. I note that in the asymmetric mode, the only contribution to friction is from modes where the center of mass of the molecule is not moving, even though segments of the molecule are stretched and compressed. Thus I attribute the friction to "internal" friction.

The FWLC model produces a prediction for the internal friction (equation (8.5)) that can be compared with the data. There is one fitting parameter $\zeta_{\mathrm{B}}$, the bending internal friction coefficient, that resists bending vibrations of the chain. Figure 8-8 shows the best fit to the data using $\zeta_{\mathrm{B}}=11 \mu \mathrm{g} \mathrm{kHz} \mathrm{nm}{ }^{3}$. There is no literature value for comparison, but the functional form is appropriate for the measurements. 


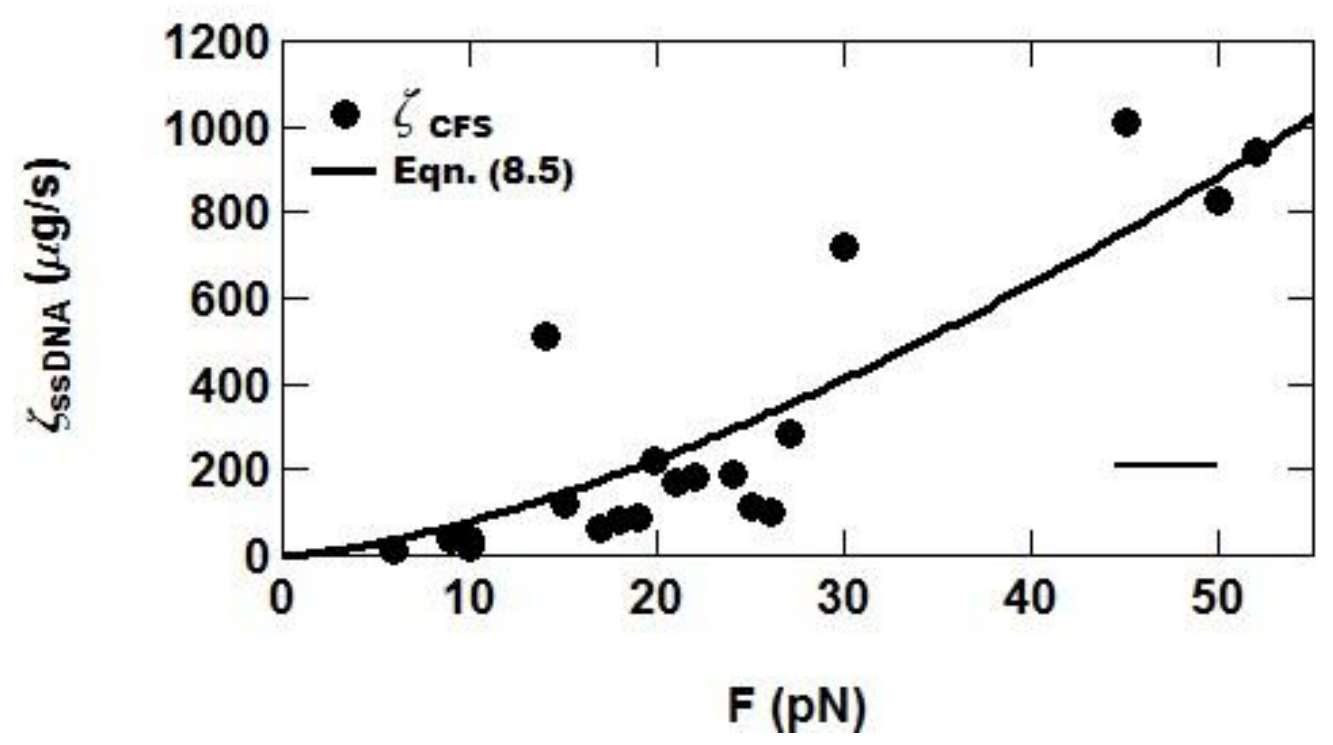

Figure 8-8: Internal friction coefficient of ssDNA measured in dynamic force-clamp mode of CFS. Solid line is fit of FWLC model equation (8.5) with $R_{\max }=42 \mathrm{~nm}$ and $l_{\mathrm{p}}=2.5 \mathrm{~nm}$ and fitted value $\zeta_{\mathrm{B}}=11 \mu \mathrm{g} \mathrm{kHzn^{3 }}$. The horizontal line shows the error in force. The error in damping is unknown as we cannot do repeat measurements of the same chain.

\section{Discussion}

It was suggested in the literature that the mechanics of nucleic acids of mixed sequences is different from homopolymeric nucleic acids due to different base stacking properties [31, 135, 136, 139]. Here I showed that homopolymeric and mixed sequences of ssDNA have similar persistence length. I chose to use short strands to minimize the probability of formation of higher order structures due to base pairing along the chain of ssDNA. It was recently suggested that tethering a chain at one or both ends or vicinity of molecular tethers can cause variations in measured flexibility. Since tethering is a requirement in all mechanical SMFS, this issue needs attention and can be addressed for example with molecular simulations [139, 140].

The bending relaxation time of the ssDNA can be found from the ratio of internal friction to stiffness, $\tau=\zeta_{\mathrm{ssDNA}} / k_{\mathrm{ssDNA}}$. Taking this ratio, I find that the relaxation time is approximately independent of force and is $\sim 0.03 \mathrm{~ms}$ (Figure 8-9). This is consistent with a theoretical 
treatment by Khatri et al. [50], where it is obvious from the ratio of equation (8.5) to equation (8.4), that the relaxation time of a WLC is independent of force. This is also consistent with a more detailed theoretical treatment by Hiraiwa and Ohta [58]: at frequencies less than $1 \mathrm{MHz}$ and forces larger than $1 \mathrm{pN}$, the relaxation time of a WLC is force-independent.

The Rouse relaxation time of a similar chain is (i.e. $\tau_{1}$ in equation (3.29)) [56]:

$$
\tau_{\mathrm{R}}=\frac{N^{2} b^{2} \zeta_{\mathrm{b}}}{3 \pi^{2} k_{\mathrm{B}} T},
$$

which gives $\tau_{\mathrm{R}} \sim 0.7 \mu \mathrm{s}$, or about $10^{2}$ times faster. It has been suggested by molecular dynamics simulation that the slow relaxation of single molecules in mechanical SMFS is an artifact due to anchoring the ends of the molecule [146]. While this is plausible until further experiments reveal more evidence, two orders of magnitude lagging in relaxation can be due to real underlying molecular events. Such slowness in relaxation time (or diffusion) was also observed in ensemble techniques such as laser-temperature jump as discussed in Chapter 3.

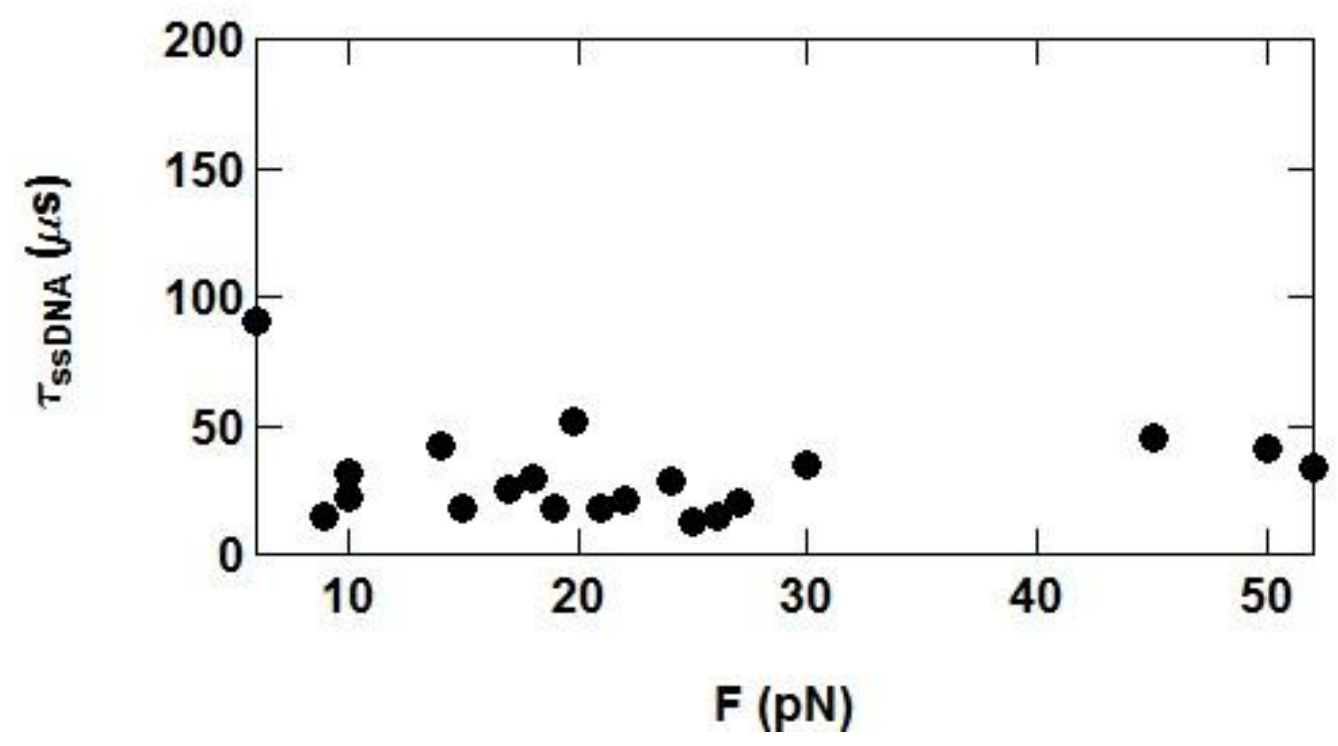

Figure 8-9: Relaxation time of ssDNA calculated from the ratio of $\zeta_{\mathrm{ssDNA}}$ to $k_{\mathrm{ssDNA}}$.

The Einstein's relation: 


$$
D=\frac{k_{\mathrm{B}} T}{\zeta}
$$

can be used in conjunction with Zwanzig's theory of unfolding-folding in a rough energy potential landscape (see also equation (3.35)) [59]:

$$
D^{*}=D \mathrm{e}^{-\left(\varepsilon / k_{\mathrm{B}} T\right)^{2}},
$$

to relate the diffusion coefficient in the rough potential $D^{*}$ to internal friction $\zeta_{\mathrm{ssDNA}}$, and diffusion coefficient in a smooth potential $D$ to solvent friction $\zeta_{\mathrm{R}}$ :

$$
\frac{\zeta_{\mathrm{ssDNA}}}{\zeta_{\mathrm{R}}}=e^{\left(\varepsilon / k_{\mathrm{B}} T\right)^{2}} .
$$

Using experimental $\zeta_{\text {ssDNA }}$ in Figure 8-8 gives:

$$
\varepsilon_{\mathrm{ssNA}}=\left\{\begin{array}{ll}
2 k_{\mathrm{B}} T & \text { at } 10 \mathrm{pN} \\
3 k_{\mathrm{B}} T & \text { at } 50 \mathrm{pN}
\end{array}\right. \text {. }
$$

A comparison between my results on roughness of unfolding-folding energy landscape of ssDNA and those obtained with different measurement techniques and molecules is shown Table 8-1. The roughness $\varepsilon$ is about 1 to $4 k_{\mathrm{B}} T$ in all cases studied. In case of proteins, it is suggested that intrachain collisions is the main source of roughness in energy landscape $[65,70,147]$. In my experiments, intrachain collisions might occur but are rare since the molecule is unfolded and there is no compact region. I attribute the observed roughness in ssDNA unfolding-folding to intrachain interactions such as pi-stacking of bases and formation of weak hydrogen bonds between bases. Such intrachain hydrogen bonds can also occur in proteins, and interestingly, the close agreement between results shown in Table 8-1 might suggest that this is the main source of roughness in all cases. A counter argument is suggested by the results of Liu at al. [64] that suggested the source of roughness in unfolding-folding of protein FiP35 is hydrophobic forces. The change in magnitude of roughness $\Delta \varepsilon$ (corresponding to magnitude of hydrophobic forces) with temperature agrees with this interpretation. Muramaya et al. [148, 149] used results on compact DNA to suggest that intrachain collisions are the source of energy landscape roughness. The close agreement between different experiments shown in Table 8-1 is interesting, but calls for more experiments. 
Table 8-1: Roughness of energy landscape in unfolding-folding of molecules.

\begin{tabular}{|c|c|c|c|}
\hline Molecule & $\varepsilon / k_{\mathrm{B}} T$ & $\begin{array}{l}\text { Measurement } \\
\text { technique }\end{array}$ & \\
\hline Myoglobin & 1.6 & $\begin{array}{l}\text { Photolysis and } \\
\text { optical adsorption }\end{array}$ & Ansari, et al. (1992) [70] \\
\hline $\begin{array}{l}\text { Tryptophan-cysteine } \\
\text { contact formation } \\
\text { (polypeptide contact) }\end{array}$ & 1.6 & $\begin{array}{l}\text { Photolysis and } \\
\text { optical adsorption }\end{array}$ & Lapidus, et al. (2000) [150] \\
\hline DNA & 2.5 & OT & $\begin{array}{l}\text { Murayama, et al. (2003) [148]; } \\
\text { Murayama, et al. (2007) [149] (see also } \\
\text { Alexander-Katz, et al. (2009) [151]) }\end{array}$ \\
\hline Beta peptide trpzip2 & 0.8 & $\begin{array}{l}\text { Laser temperature } \\
\text { jump }\end{array}$ & Yang and Gruebele (2004) [147] \\
\hline Protein ddFLN4 & 4 & AFM & Schlierf and Rief (2005) [65] \\
\hline Protein FiP35 & $\begin{array}{l}\Delta \approx 0.5 \\
\mathrm{~T}=60 \text { to } \\
83^{\circ} \mathrm{C}\end{array}$ & $\begin{array}{l}\text { Laser temperature } \\
\text { jump }\end{array}$ & Liu, et al. (2009) [64] \\
\hline $\begin{array}{l}\text { R16 and R17 domains of } \\
\alpha \text {-spectrin }\end{array}$ & $\begin{array}{l}1.2 \text { for R16, } \\
1.6 \text { for R } 17\end{array}$ & Chevron plots & Wensley, et al. (2010) [63] \\
\hline ssDNA & $\begin{array}{l}2 \text { at } 10 \mathrm{pN} \\
3 \text { at } 50 \mathrm{pN}\end{array}$ & $\mathrm{CFS}^{1}$ & \\
\hline
\end{tabular}

${ }^{1}$ current thesis

\section{Summary}

Since short ssDNA is ubiquitous in biological processes (for example during DNA replication) its mechanical properties are of great interest. In this chapter I used correlation force spectroscopy and atomic force microscopy to extend the previous investigations on mechanics of ssDNA to short ssDNA of mixed sequences. The persistence length of mixed sequences was found to be about the same as for homopolymer sequences, about $2.5 \mathrm{~nm}$. The functional form of the stiffness-load and internal friction-load responses were consistent with the worm-like- 
chain model. The internal friction of DNA is also important because it sets the rate at which changes in conformation can occur. The CFS measurements described here enabled the first measurement of the internal friction coefficient of ssDNA and provide an estimate of the bending internal friction of $\zeta_{\mathrm{B}}=11 \mu \mathrm{g} \mathrm{kHz} \mathrm{nm}{ }^{3}$. The bending relaxation time of ssDNA is $\sim 0.03 \mathrm{~ms}$, independent of force, which is consistent with the predictions of [58]. I also calculated the roughness of energy landscape in unfolding-folding process of ssDNA using Zwanzig's theory and found $\varepsilon_{\mathrm{sSNA}}=2$ to $3 k_{\mathrm{B}} T$, consistent with previous studies. 


\section{Summary}

The idea of cross-correlating the thermal motions of two objects to determine the properties of the intervening material was first used in optical tweezers measurements of two microparticles [108], and an extension to two microcantilevers was first suggested by Roukes [Arlett, et al., Lecture Notes in Physics, 2007]. The method of analyses via the fluctuation-dissipation theorem for analyzing the cross-correlation was described by Paul and Cross [Paul and Cross, PRL, 2004]. This thesis describes the first experimental realization of such a device, correlation force spectroscopy. It was also shown that CFS can be used as a viscometer (see for example Figure 4-2) and a single molecule force spectrometer (see for example Figure 8-7 and Figure 8-8). The main advantages of CFS over the conventional atomic force microscopy (AFM) is the lower thermal noise that is essential in single molecule force measurements (see Figure 2-1) and the direct accessibility of the mechanics of asymmetric modes that can reveal properties such as the internal friction. The internal friction controls the time required for the rearrangement of polymers, including DNA and proteins.

\section{An Overview of Chapters}

Chapter 2 described the analysis of thermal fluctuations to mechanical properties that is based on the fluctuation-dissipation theorem (equation (2.4) which was experimentally validated in Figure 2-7 and equation (2.5)). In Chapter 4, correlation experiments were shown in laterally offset CFS (see Figure 2-3) to characterize fluid density and viscosity. It was shown that the crosscorrelation between two cantilevers is a function of fluid properties and thus CFS can be used as a viscometer (Figure 4-2). I developed the microrheology application of CFS to vertically offset configuration by attaching a micro-sphere to distal end of each cantilever (see Figure 5-1). Interesting results were obtained for fluid motion in the gap of the two cantilevers. In Chapter 6 the vertically offset CFS was used to measure the correlations between two cantilevers that are vertically offset (Figure 6-1). The experiments in this chapter were precursor to single molecule force spectroscopy experiments. It was specifically shown in this chapter that CFS has a lower 
noise floor than conventional AFM single cantilever measurements, and is thus a better spectroscopy tool for molecular event with low forces (see Figure 6-5). Single molecule measurements of dextran in Chapter 7 showed that in order to successfully perform such measurements some improvements on the instrument and experimental methods are vital; mainly $i$. to perform the experiments in a closed fluid cell to avoid contamination, and specifically, ii. to anchor the molecules on the surface via chemical reaction. It also showed that CFS, unlike conventional AFM that only gives single molecule stiffness, gives molecule stiffness and friction (Figure 7-7). In Chapter 8, I performed single molecule measurements on single stranded DNA where each molecule was anchored to surface through gold-thiol reaction and the experiments were performed in a closed fluid cell. The resulting molecular stiffness (Figure 8-7) and internal friction (Figure 8-8) were fit to theories of elasticity (equation (8.1) or (8.4)) and friction (equation (8.5)) for a worm-like chain model and gave interesting physical features about the dynamics of ssDNA.

\section{Future Work}

The latest version of the vertically offset CFS that is in a closed fluid cell provides opportunity to perform single molecule measurements while actively controlling the medium (e.g. composition, $\mathrm{pH}$, ionic strength) and temperature. These parameters can each have very interesting molecular level effects on the dynamics of single molecules that we need to study and understand. This is the essence of Francis Crick's statement that I recalled at the introduction to this thesis.

An example of an experiment that comes up immediately is the investigation of the effect of the internal structure of a protein on its elasticity and more importantly internal friction. The internal friction of a protein is a measure of its energy landscape roughness that slows down its folding. On one hand being in the unfolded state for a protein for a longer time increase its chance of degradation that causes disease [63]. On the other hand fast folders (with smooth energy landscape) are prone to aggregation and proteolysis [152]. This suggests that the nature has provided these molecules with an optimum roughness on the energy landscape for an optimal reconfiguration pace and function. This brings about an opportunity for single molecule techniques such as CFS to explore this roughness (as well as elasticity) of proteins. Numbers of 
known proteins are limitless but good starting points are proteins that have been studied in ensemble techniques, for example $\alpha$-spectrin, $\alpha$-synuclein and protein FiP35. Amyloid forming proteins are also very interesting, e.g. Amyloid A, that are implicated in protein misfolding diseases such as Parkinsons Disease, Alzheimer's, Parkinson's, or diabetes [153]. To tether a protein between the tips of two cantilevers in CFS and to extend it to a known force and conformation, observing its folding while simultaneously collecting its stiffness and friction, results in a correlation between function and roughness, and a correlation between function and elasticity of the protein. Measuring the effect of temperature and solvent properties in this case is useful in pharmaceutical applications.

One interesting case to be studied is interactions between DNA and DNA-binding proteins. A motivation for this study comes from the important role of specific proteins in regulation and catalysis of multiple DNA tasks, such as replication, transcription, compaction, unwinding, chemical modification, and sequence repair [154]. In cells, DNA is constantly twisted, bent and stretched by numerous proteins mediating genome transactions. Understanding these essential biological processes requires in-depth knowledge of how DNA complies to mechanical stress due to binding with proteins [155]. Protein binding to DNA alters the conformation of DNA and thus function. For example, the high mobility group B protein (HMGB) when binds to DNA enhances its flexibility by decreasing DNA persistence length [154]. The affinity of proteinDNA binding, that we describe using mechanical terms of stiffness and friction, are vital in cancer treatment studies [154]. Using CFS the dynamics of protein binding and unbinding and its interpretation in terms of mechanical properties can be obtained. Invaluable insights are gained from these experiments.

Yet obscure in many experimental investigations is the molecule origin of internal friction. It is speculated that multiple effects can result in roughness of conformational changes and thus internal friction. To elucidate on the effect of internal bonding and conformations, intrinsically disordered proteins such as p53 and BRCA1 can be used which have a lot of internal structure. DNA and RNA and single stranded DNA exhibit a different origin of internal friction that is due to internal bonding between segments that are totally exposed to the solvent. I started this study using single stranded DNA but more is to be done on DNA and RNA. 
These studies are not limited to biological molecules. For synthetic molecules, the active temperature control provides transitions to various conformations prior to application of force due to changing of the quality of solvent from A-thermal to $\theta$-solvent to non-solvent. A thermal energy landscape can be produced for the molecular stiffness and friction as a function of temperature.

Nevertheless, the success of all these possible experiments is due to careful immobilization of molecules on surface. If possible, non-specific adsorption of molecules to surface is to be avoided in all cases. Surfaces should be passivated by appropriate agents to reduce the possibility of surface-to-surface adhesion. Another challenge is to anchor a single chain of molecule between two cantilevers. Due to weak tethers used in ssDNA experiments shown in the Chapter 8, I could not clamp the molecule for so long. The greater the duration for which the molecule is tethered between the two cantilevers, the longer will be the duration of data acquisition and thus the higher will be the signal to noise ratio. I used hydrogen binding of $20 \mathrm{~A}$ and $\mathrm{T}$ bases to clamp ssDNA between the two cantilevers, a longer ds region leads to exponentially longer clamping times. Even stronger binding, such ligand-receptor type is possible, and would give much better signal to noise.

With respect to application of CFS in microrheology, I would recommend using the colloidal probe CFS (to laterally offset CFS) since analytic solution to interparticle hydrodynamics is already available. Thin film of fluids, from nanometer to micrometer length scale, can be explored over a wide frequency range in this setup. Such studies are essential for example in nanofluids [156] and polymer solutions with heterogeneous properties.

Recall that the main advantage of CFS over AFM was the lower hydrodynamic friction (that reduced the thermal noise) as well as cross-correlation of the two fluctuation signal (that enhances force sensitivity). The former benefit can be easily obtained by placing the cantilever against an edge. The hydrodynamic friction in this configuration is much smaller than the cantilever against an entire surface. I performed such measurement when I was evaluating the fluctuation-dissipation theorem for one cantilever as presented in Chapter 2 for validation of 
fluctuation-dissipation theorem for one cantilever. This is an interesting experiment although it has the trade-off of lower sensitivity (since this is autocorrelation of one cantilever as compared to cross-correlation of two cantilevers in CFS) but easier operation. Both single molecule force spectroscopy and microrheology can be performed in this configuration and results compared with similar experiment in CFS. I would recommend, depending on the experiment, that if higher sensitivity is required experiments are to be performed in a CFS apparatus, however if single cantilever against an edge has enough sensitivity due to its ease of operation and access to an entire edge it is the preferable experimental approach. 


\section{Appendix I: Simple Harmonic Oscillator Model for Laterally Offset Correlation Force Spectroscopy}

Equations (4.1) and (4.2) are the dynamic equations of motion for the system shown in Figure 4-3. Initially two new coordinate systems are introduced. Each coordinate represent a mode in coupled motion of the masses:

$$
\begin{aligned}
& X_{\mathrm{s}}=X_{1}+X_{2}, \\
& X_{\mathrm{a}}=X_{1}-X_{2} .
\end{aligned}
$$

$X_{\mathrm{s}}$ is the mode where the vibrations are in phase (the symmetric vibration) and $X_{\mathrm{a}}$ is the mode when the vibrations are out of phase (the antisymmetric stretch). The equations of motion in the new coordinate system are:

$$
\begin{gathered}
\ddot{X}_{\mathrm{s}}+\left(\frac{\gamma_{\mathrm{a}}}{m}+\frac{2 \gamma_{\mathrm{c}}}{m}\right) \dot{X}_{\mathrm{s}}+\omega_{\mathrm{r}}^{2} X_{\mathrm{s}}=0, \\
\ddot{X}_{\mathrm{a}}+\frac{\gamma_{\mathrm{a}}}{m} \dot{X}_{\mathrm{a}}+\omega_{\mathrm{r}}^{2} X_{\mathrm{a}}=0,
\end{gathered}
$$

where the solutions are:

$$
\begin{aligned}
& X_{\mathrm{s}}=\frac{-1}{\left(n_{+x}-n_{-x}\right)}\left(n_{-x} \exp \left(n_{+x} t\right)-n_{+x} \exp \left(n_{-x} t\right)\right), \\
& X_{\mathrm{a}}=\frac{-1}{\left(n_{+y}-n_{-y}\right)}\left(n_{-y} \exp \left(n_{+y} t\right)-n_{+y} \exp \left(n_{-y} t\right)\right) .
\end{aligned}
$$

In terms of cantilevers' fluctuations, the solutions form:

$$
X_{1}=\frac{1}{2}\left[\begin{array}{l}
\frac{-1}{\left(n_{+x}-n_{-x}\right)}\left(n_{-x} \exp \left(n_{+x} t\right)-n_{+x} \exp \left(n_{-x} t\right)\right)+ \\
\frac{-1}{\left(n_{+y}-n_{-y}\right)}\left(n_{-y} \exp \left(n_{+y} t\right)-n_{+y} \exp \left(n_{-y} t\right)\right)
\end{array}\right],
$$




$$
X_{2}=\frac{1}{2}\left[\begin{array}{l}
\frac{-1}{\left(n_{+x}-n_{-x}\right)}\left(n_{-x} \exp \left(n_{+x} t\right)-n_{+x} \exp \left(n_{-x} t\right)\right)- \\
\frac{-1}{\left(n_{+y}-n_{-y}\right)}\left(n_{-y} \exp \left(n_{+y} t\right)-n_{+y} \exp \left(n_{-y} t\right)\right)
\end{array}\right] .
$$

The coefficients are as follows:

$$
\begin{aligned}
& c_{\mathrm{x}}^{*}=\frac{\gamma_{\mathrm{a}}}{m}+\frac{2 \gamma_{\mathrm{c}}}{m}, \\
& c_{y}^{*}=\frac{\gamma_{a}}{m}, \\
& k_{x}^{*}=k_{y}^{*}=\omega_{r}^{2}, \\
& n_{+x,-x}=\frac{-c_{x}^{*} \pm \sqrt{c_{x}^{* 2}-4 k_{x}^{*}}}{2}, \\
& n_{+y,-y}=\frac{-c_{y}^{*} \pm \sqrt{c_{y}^{*^{2}}-4 k_{y}^{*}}}{2} .
\end{aligned}
$$

Inserting the above expressions into the expressions for $X_{1}$ and $X_{2}$ results in equations (4.3) and (4.4). The noise spectrum can be obtained using equations (13) and (14) in [43] as follows:

$$
\begin{gathered}
G_{11,22}(\omega)=2 k_{B} T\left[\left(\frac{\left(\gamma_{\mathrm{a}}+2 \gamma_{\mathrm{c}}\right)}{\left(m(2 \pi \omega)^{2}-k\right)^{2}+\left(\gamma_{\mathrm{a}}+2 \gamma_{\mathrm{c}}\right)^{2}(2 \pi \omega)^{2}}\right)+\left(\frac{\gamma_{\mathrm{a}}}{\left(m(2 \pi \omega)^{2}-k\right)^{2}+\left(\gamma_{\mathrm{a}}\right)^{2}(2 \pi \omega)^{2}}\right)\right], \\
G_{12}(\omega)=2 k_{B} T\left[\left(\frac{\left.\gamma_{\mathrm{a}}+2 \gamma_{\mathrm{c}}\right)}{\left(m(2 \pi \omega)^{2}-k\right)^{2}+\left(\gamma_{\mathrm{a}}+2 \gamma_{\mathrm{c}}\right)^{2}(2 \pi \omega)^{2}}\right)-\left(\frac{\gamma_{\mathrm{a}}}{\left(m(2 \pi \omega)^{2}-k\right)^{2}+\left(\gamma_{\mathrm{a}}\right)^{2}(2 \pi \omega)^{2}}\right)\right] .
\end{gathered}
$$




\section{Appendix II: Simple Harmonic Oscillator Model for Vertically Offset Correlation Force Spectroscopy with Tethered Molecule}

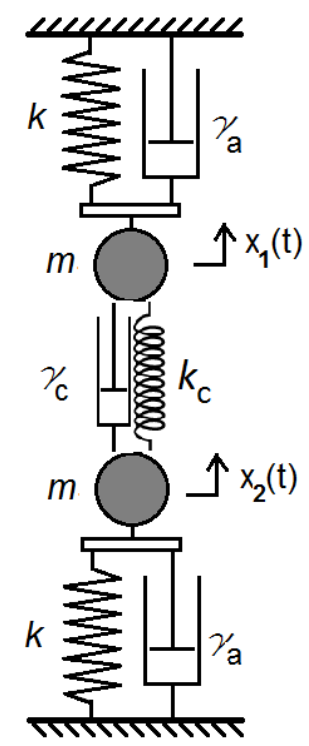

Figure: Schematic of a cantilever pair modeled as mass-spring-damper system with the tethered molecule modeled as a spring.

The dynamic equation of motion for the system shown in the above figure is as follows:

$$
\begin{aligned}
& m \ddot{X}_{1}=-\gamma_{\mathrm{a}} \dot{X}_{1}-k_{\mathrm{c}} X_{1}-\gamma_{\mathrm{c}}\left(\dot{X}_{1}-\dot{X}_{2}\right)-k_{\mathrm{p}}\left(X_{1}-X_{2}\right), \\
& m \ddot{X}_{2}=-\gamma_{\mathrm{a}} \dot{X}_{2}-k_{\mathrm{c}} X_{2}+\gamma_{\mathrm{c}}\left(\dot{X}_{1}-\dot{X}_{2}\right)+k_{\mathrm{p}}\left(X_{1}-X_{2}\right) .
\end{aligned}
$$

The symmetric mode $\left(X_{1}+X_{2}=X_{s}\right)$ gets the following form:

$$
\ddot{X}_{\mathrm{s}}+\left(\frac{\gamma_{a}}{m}\right) \dot{X}_{\mathrm{s}}+\omega_{r}^{2} X_{\mathrm{s}}=0,
$$

and the anti-symmetric mode $\left(X_{1}-X_{2}=X_{\mathrm{a}}\right)$ gets the following form: 


$$
\ddot{X}_{\mathrm{a}}+\left(\frac{\gamma_{a}+2 \gamma_{\mathrm{c}}}{m}\right) \dot{X}_{\mathrm{a}}+\left(\omega_{r}^{2}+\frac{2 k_{\mathrm{p}}}{m}\right) X_{\mathrm{a}}=0
$$

where the solutions are:

$$
\begin{gathered}
X_{s}(t)=\frac{k_{c}+2 k_{p}}{k_{c}+k_{p}} \exp \left(\frac{-\gamma_{a}}{2 m} t\right)\left[\cos \left(\sqrt{\omega_{r}^{2}-\left(\frac{\gamma_{a}}{2 m}\right)^{2}} t\right)+\frac{\frac{\gamma_{a}}{2 m}}{\sqrt{\omega_{r}^{2}-\left(\frac{\gamma_{a}}{2 m}\right)^{2}}} \sin \left(\sqrt{\omega_{r}^{2}-\left(\frac{\gamma_{a}}{2 m}\right)^{2}} t\right)\right. \\
X_{a}(t)=\frac{k_{c}}{k_{c}+k_{p}} \exp \left(-\frac{\gamma_{a}+2 \gamma_{c}}{2 m} t\right)\left[\frac{\cos \left(\sqrt{\left(\omega_{r}^{2}+\frac{2 k_{\mathrm{p}}}{m}\right)-\left(\frac{\gamma_{a}+2 \gamma_{c}}{2 m}\right)^{2}} t\right)+}{\frac{\gamma_{a}+2 \gamma_{c}}{2 m}}\right]\left[\begin{array}{l}
\sqrt{\left(\omega_{r}^{2}+\frac{2 k_{\mathrm{p}}}{m}\right)-\left(\frac{\gamma_{a}+2 \gamma_{c}}{2 m}\right)^{2}} \\
\left.\sin \left(\sqrt{\left(\omega_{r}^{2}+\frac{2 k_{\mathrm{p}}}{m}\right)-\left(\frac{\gamma_{a}+2 \gamma_{c}}{2 m}\right)^{2}} t\right)\right] .
\end{array}\right]
\end{gathered}
$$

Applying equations (13) and (14) in [43], in frequency domain equations (7.7) and (7.8) can be obtained. 


\section{References}

1. Clausen-Schaumann, H., et al., Force spectroscopy with single bio-molecules. Current Opinion in Chemical Biology, 2000. 4(5): p. 524-530.

2. $\quad$ Stryer, L., Biochemistry. 1981, SAN FRANCISCO: WH Freeman and Company.

3. Hanley, W., et al., Single molecule characterization of P-selectin/ligand binding. Journal of Biological Chemistry, 2003. 278(12): p. 10556-10561.

4. Serpell, L.C., Alzheimer's amyloid fibrils: structure and assembly. Biochimica Et Biophysica ActaMolecular Basis of Disease, 2000. 1502(1): p. 16-30.

5. Sunde, M. and C.C.F. Blake, From the globular to the fibrous state: protein structure and structural conversion in amyloid formation. Quarterly Reviews of Biophysics, 1998. 31(1): p. 1-39.

6. Calamai, M., F. Chiti, and C.M. Dobson, Amyloid fibril formation can proceed from different conformations of a partially unfolded protein. Biophysical Journal, 2005. 89(6): p. 4201-4210.

7. Dobson, C.M., Protein folding and misfolding. Nature, 2003. 426(6968): p. 884-890.

8. Szoszkiewicz, R., Force-extension and force-clamp AFM spectroscopies in investigating mechanochemical reactions and mechanical properties of single biomolecules, in scanning probe microscopy in nanoscience and nanotechnology, B. Bharat, Editor. 2010, Springer: BERLIN. p. 395-423.

9. Michaelis, J., et al., DNA based molecular motors. Physics of Life Reviews, 2009. 6(4): p. 250-266.

10. Kishino, A. and T. Yanagida, Force measurements by micromanipulation of a single actin filament by glass needles. Nature, 1988. 334(6177): p. 74-76.

11. Evans, E., K. Ritchie, and R. Merkel, Sensitive force technique to probe molecular adhesion and structural linkages at biological interfaces. Biophysical Journal, 1995. 68(6): p. 2580-2587.

12. Meiners, J.C. and S.R. Quake, Femtonewton force spectroscopy of single extended DNA molecules. Physical Review Letters, 2000. 84(21): p. 5014-5017.

13. Bao, X.R., H.J. Lee, and S.R. Quake, Behavior of complex knots in single DNA molecules. Physical Review Letters, 2003. 91(26).

14. Salomo, M., et al., Binding of TmHU to single dsDNA as observed by optical tweezers. Journal of Molecular Biology, 2006. 359(3): p. 769-776.

15. Salomo, M., et al., The elastic properties of single double-stranded DNA chains of different lengths as measured with optical tweezers. Colloid and Polymer Science, 2006. 284(11): p. 1325-1331.

16. Chen, Y.F., G.A. Blab, and J.C. Meiners, Stretching submicron biomolecules with constant-force axial optical tweezers. Biophysical Journal, 2009. 96(11): p. 4701-4708.

17. Kegler, K., M. Salomo, and F. Kremer, Forces of interaction between DNA-grafted colloids: An optical tweezer measurement. Physical Review Letters, 2007. 98(5).

18. Svoboda, K., et al., Direct observation of kinesin stepping by optical trapping interferometry. Nature, 1993. 365(6448): p. 721-727.

19. Abbondanzieri, E.A., et al., Direct observation of base-pair stepping by RNA polymerase. Nature, 2005. 438(7067): p. 460-465.

20. Idiris, A., et al., Force measurement for antigen-antibody interaction by atomic force microscopy using a photograft-polymer spacer. Biomacromolecules, 2005. 6(5): p. 2776-2784.

21. Hinterdorfer, P., et al., Detection and localization of individual antibody-antigen recognition events by atomic force microscopy. Proceedings of the National Academy of Sciences, 1996. 93(8): p. 3477-3481.

22. Lee, G.U., L.A. Chrisey, and R.J. Colton, Direct measurement of the forces between complementary strands of DNA. Science, 1994. 266(5186): p. 771-773.

23. Rief, M., et al., Reversible unfolding of individual titin immunoglobulin domains by AFM. Science, 1997. 276(5315): p. 1109-1112.

24. Fisher, T.E., P.E. Marszalek, and J.M. Fernandez, Stretching single molecules into novel conformations using the atomic force microscope. Nature Structural Biology, 2000. 7(9): p. 719-724.

25. Moy, V.T., E.L. Florin, and H.E. Gaub, Intermolecular forces and energies between ligands and receptors. Science, 1994. 266(5183): p. 257-259. 
26. Evans, E. and K. Ritchie, Dynamic strength of molecular adhesion bonds. Biophysical Journal, 1997. 72(4): p. 1541-1555.

27. Merkel, R., et al., Energy landscapes of receptor-ligand bonds explored with dynamic force spectroscopy. Nature, 1999. 397(6714): p. 50-53.

28. Schlierf, M., H.B. Li, and J.M. Fernandez, The unfolding kinetics of ubiquitin captured with singlemolecule force-clamp techniques. Proceedings of the National Academy of Sciences, 2004. 101(19): p. 7299-7304.

29. Boland, T. and B.D. Ratner, Direct measurement of hydrogen-bonding in DNA nucleotide bases by atomicforce microscopy. Proceedings of the National Academy of Sciences, 1995. 92(12): p. 5297-5301.

30. EssevazRoulet, B., U. Bockelmann, and F. Heslot, Mechanical separation of the complementary strands of DNA. Proceedings of the National Academy of Sciences, 1997. 94(22): p. 11935-11940.

31. Rief, M., H. Clausen-Schaumann, and H.E. Gaub, Sequence-dependent mechanics of single DNA molecules. Nature Structural Biology, 1999. 6(4): p. 346-349.

32. Krautbauer, R., M. Rief, and H.E. Gaub, Unzipping DNA oligomers. Nano Letters, 2003. 3(4): p. 493-496.

33. Fernandez, J.M. and H.B. Li, Force-clamp spectroscopy monitors the folding trajectory of a single protein. Science, 2004. 303(5664): p. 1674-1678.

34. Dey, A. and R. Szoszkiewicz, Complete noise analysis of a simple force spectroscopy AFM setup and its applications to study nanomechanics of mammalian Notch 1 protein. Nanotechnology, 2012. 23(17).

35. Oberhauser, A.F., et al., Stepwise unfolding of titin under force-clamp atomic force microscopy. Proceedings of the National Academy of Sciences, 2001. 98(2): p. 468-472.

36. Kawakami, M., et al., Viscoelastic properties of single polysaccharide molecules determined by analysis of thermally driven oscillations of an atomic force microscope cantilever. Langmuir, 2004. 20(21): p. 92999303.

37. Bippes, C.A., et al., Direct measurement of single-molecule visco-elasticity in atomic force microscope force-extension experiments. European Biophysics Journal, 2006. 35(3): p. 287-292.

38. Kawakami, M., et al., Viscoelastic properties of single poly(ethylene glycol) molecules. A European Journal of Chemical Physics and Physical Chemistry, 2006. 7(8): p. 1710-1716.

39. Kawakami, M., et al., Viscoelastic measurements of single molecules on a millisecond time scale by magnetically driven oscillation of an atomic force microscope cantilever. Langmuir, 2005. 21(10): p. 47654772.

40. Janovjak, H., D.J. Muller, and A.D.L. Humphris, Molecular force modulation spectroscopy revealing the dynamic response of single bacteriorhodopsins. Biophysical Journal, 2005. 88(2): p. 1423-1431.

41. Zlatanova, J., S.M. Lindsay, and S.H. Leuba, Single molecule force spectroscopy in biology using the atomic force microscope. Progress in Biophysics \& Molecular Biology, 2000. 74(1-2): p. 37-61.

42. Paul, M.R. and M.C. Cross, Stochastic dynamics of nanoscale mechanical oscillators immersed in a viscous fluid. Physical Review Letters, 2004. 92(23).

43. Paul, M.R., M.T. Clark, and M.C. Cross, The stochastic dynamics of micron and nanoscale elastic cantilevers in fluid: Fluctuations from dissipation. Nanotechnology, 2006. 17(17): p. 4502-4513.

44. Greenleaf, W.J., M.T. Woodside, and S.M. Block, High-resolution, single-molecule measurements of biomolecular motion, in Annual Review of Biophysics and Biomolecular Structure. 2007, Annual Reviews: PALO ALTO, CALIFORNIA. p. 171-190.

45. Boskovic, S., et al., Rheological measurements using microcantilevers. Journal of Rheology, 2002. 46(4): p. 891-899.

46. Radiom, M., et al., Rheology of fluids measured by correlation force spectroscopy. Review of Scientific Instruments, 2012. 83(4).

47. Arlett, J.L., et al., BioNEMS: Nanomechanical systems for single-molecule biophysics, in Controlled Nanoscale Motion, H. Linke and A. Mansson, Editors. 2007, Springer-Verlag: BERLIN. p. 241-270.

48. Chandler, D., Introduction to modern statistical mechanics. 1987, NEW YORK: Oxford University Press. 288.

49. Rubinstein, M. and R.H. Colby, Polymer physics. 2003, NEW YORK: Oxford University Press. 440.

50. Khatri, B.S., et al., Internal friction of single polypeptide chains at high stretch. Faraday Discussions, 2008. 139: p. 35-51.

51. Murphy, M.C., et al., Probing single-stranded DNA conformational flexibility using fluorescence spectroscopy. Biophysical Journal, 2004. 86(4): p. 2530-2537. 
52. Winkler, R.G., Analytical calculation of the relaxation dynamics of partially stretched flexible chain molecules: Necessity of a wormlike chain description. Physical Review Letters, 1999. 82(9): p. 1843-1846.

53. Thirumalai, D. and B.Y. Ha, Statistical mechanics of semiflexible chains, in Theoretical and Mathematical Models in Polymer Research, A. Grosberg, Editor. 1998, Academic Press: SAN DIEGO. p. 1-35.

54. Marko, J.F. and E.D. Siggia, Stretching DNA. Macromolecules, 1995. 28(26): p. 8759-8770.

55. de Gennes, P.G., Introduction to Polymer Dynamics. 1990, NEW YORK: Cambridge University Press.

56. Doi, M. and S.F. Edwards, The theory of polymer dynamics. International Series of monographs on physics. 1998, NEW YORK: Oxford University Press.

57. Khatri, B., Theory of single biomolecule conformational viscoelasticity, in School of Physocs and Astronomy. 2006, The University of Leeds.

58. Hiraiwa, T. and T. Ohta, Linear viscoelasticity of a single semiflexible polymer with internal friction. Journal of Chemical Physics, 2010. 133(4).

59. Zwanzig, R., Diffusion in a rough potential. Proceedings of the National Academy of Sciences of the United States of America, 1988. 85(7): p. 2029-2030.

60. Hyeon, C.B. and D. Thirumalai, Can energy landscape roughness of proteins and RNA be measured by using mechanical unfolding experiments? Proceedings of the National Academy of Sciences, 2003. 100(18): p. 10249-10253.

61. Soranno, A., et al., Quantifying internal friction in unfolded and intrinsically disordered proteins with single-molecule spectroscopy. Proceedings of the National Academy of Sciences, 2012. 109(44): p. 1780017806.

62. Kramers, H.A., Brownian motion in a field of force and the diffusion model of chemical reactions. Physica, 1940. 7(4): p. 284-304.

63. Wensley, B.G., et al., Experimental evidence for a frustrated energy landscape in a three-helix-bundle protein family. Nature, 2010. 463(7281): p. 685-689.

64. Liu, F., M. Nakaema, and M. Gruebele, The transition state transit time of WW domain folding is controlled by energy landscape roughness. Journal of Chemical Physics, 2009. 131(19).

65. Schlierf, M. and M. Rief, Temperature softening of a protein in single-molecule experiments. Journal of Molecular Biology, 2005. 354(2): p. 497-503.

66. Schulz, J.C.F., et al., Peptide chain dynamics in light and heavy water: Zooming in on internal friction. Journal of the American Chemical Society, 2012. 134(14): p. 6273-6279.

67. Khatri, B.S. and T.C.B. McLeish, Rouse model with internal friction: A coarse grained framework for single biopolymer dynamics. Macromolecules, 2007. 40(18): p. 6770-6777.

68. Cellmer, T., et al., Measuring internal friction of an ultrafast-folding protein. Proceedings of the National Academy of Sciences, 2008. 105(47): p. 18320-18325.

69. Qiu, L.L. and S.J. Hagen, A limiting speed for protein folding at low solvent viscosity. Journal of the American Chemical Society, 2004. 126(11): p. 3398-3399.

70. Ansari, A., et al., The role of solvent viscosity in the dynamics of protein conformational changes. Science, 1992. 256(5065): p. 1796-1798.

71. Khatri, B.S., et al., Entropy and barrier-controlled fluctuations determine conformational viscoelasticity of single biomolecules. Biophysical Journal, 2007. 92(6): p. 1825-1835.

72. Poirier, M.G. and J.F. Marko, Effect of internal friction on biofilament dynamics. Physical Review Letters, 2002. 88(22).

73. Gardel, M.L., M.T. Valentine, and D.A. Weitz, Microrheology, in Microscale diagnostic techniques, K. Breuer, Editor. 2005, Springer-Verlag: BERLIN. p. 1-49.

74. Ziemann, F., J. Radler, and E. Sackmann, Local measurements of viscoelastic moduli of entangled actin netweotks using an oscillating magnetic bead micro-rheometer. Biophysics Journal, 1994. 66(6): p. 22102216.

75. Mason, T.G. and D.A. Weitz, Linear viscoelasticity of colloidal hard-sphere suspensions near the glasstransition. Physical Review Letters, 1995. 75(14): p. 2770-2773.

76. Mason, T.G., et al., Particle tracking microrheology of complex fluids. Physical Review Letters, 1997. 79(17): p. 3282-3285.

77. Crocker, J.C., et al., Two-point microrheology of inhomogeneous soft materials. Physical Review Letters, 2000. 85(4): p. 888-891.

78. Brau, R.R., et al., Passive and active microrheology with optical tweezers. Journal of Optics A, 2007. 9(8): p. S103-S112. 
79. Squires, T.M. and T.G. Mason, Fluid mechanics of microrheology. Annual Review of Fluid Mechanics, 2010. 42: p. 413-438.

80. Chen, D.T., et al., Rheological microscopy: Local mechanical properties from microrheology. Physical Review Letters, 2003.90(10).

81. Gittes, F., et al., Microscopic viscoelasticity: Shear moduli of soft materials determined from thermal fluctuations. Physical Review Letter, 1997. 79(17): p. 3286-3289.

82. Buchanan, M., et al., Comparing macrorheology and one- and two-point microrheology in wormlike micelle solutions. Macromolecules, 2005. 38(21): p. 8840-8844.

83. Cardinaux, F., et al., Microrheology of giant-micelle solutions. Europhysics Letter, 2002. 57(5): p. 738744.

84. Schnurr, B., et al., Determining microscopic viscoelasticity in flexible and semiflexible polymer networks from thermal fluctuations. Macromolecules, 1997. 30(25): p. 7781-7792.

85. Liu, J., et al., Microrheology probes length scale dependent rheology. Physical Review Letters, 2006. 96(11).

86. Hennemeyer, M., S. Burghardt, and R.W. Stark, Cantilever micro-rheometer for the dcharacterization of sugar solutions. Sensors, 2008. 8(1): p. 10-22.

87. Youssry, M., et al., A straightforward determination of fluid viscosity and density using microcantilevers: from experimental data to analytical expressions. Sensors Actuators A, 2011. 172: p. 40-46.

88. Motamedi, R. and P.M. Wood-Adams, Measurement of fluid properties using acoustically excited atomic force microscope micro-cantilever. Journal of Rheology, 2010. 54(5): p. 959-980.

89. Basak, S. and A. Raman, Hydrodynamic coupling between micromechanical beams oscillating in viscous fluids. Physics of Fluids, 2007. 19(1).

90. Basak, S., A. Raman, and S.V. Garimella, Hydrodynamic loading of microcantilevers vibrating in viscous fluids. Journal of Applied Physics, 2006. 99(11).

91. Chon, J.W.M., P. Mulvaney, and J.E. Sader, Experimental validation of theoretical models for the frequency response of atomic force microscope cantilever beams immersed in fluids. Journal of Applied Physics, 2000. 87(8): p. 3978-3988.

92. Korsunsky, A.M., et al., On the micromechanics of micro-cantilever sensors: Property analysis and eigenstrain modeling. Sensors Actuators A, 2007. 139(1-2): p. 70-77.

93. Raman, A., J. Melcher, and R. Tung, Cantilever dynamics in atomic force microscopy. Nanotoday, 2008. 3(1): p. 20-27.

94. Sader, J.E., Frequency response of cantilever beams immersed in viscous fluids with applications to the atomic force microscope. Journal of Applied Physics, 1998. 84(1): p. 64-76.

95. Spletzer, M., et al., Highly sensitive mass detection and identification using vibration localization in coupled microcantilever arrays. Applied Physics Letters, 2008. 92(11).

96. Stark, R.W., T. Drobek, and W.M. Heckl, Thermomechanical noise of a free v-shaped cantilever for atomic-force microscopy. Ultramicroscopy, 2001. 86(1-2): p. 207-215.

97. Van Eysden, C.A. and J.E. Sader, Frequency response of cantilever beams immersed in viscous fluids with applications to the atomic force microscope: Arbitrary mode order. Journal of Applied Physics, 2007. 101(4).

98. Kim, S., K.D. Kihm, and T. Thundat, Fluidic applications for atomic force microscopy AFM with microcantilever sensors. Experiments in Fluids, 2010. 48(5): p. 721-736.

99. Stark, R.W., Spectroscopy of higher harmonics in dynamic atomic force microscopy. Nanotechnology, 2004. 15(3): p. 347-351.

100. Honig, C.D.F., et al., Correlations between the thermal vibrations of two cantilevers: Validation of deterministic analysis via the fluctuation-dissipation theorem. Applied Physics Letters, 2012. 100(5).

101. Stokes, G.G., On the effect of the internal friction of fluids on the motion of pendulums. Cambridge Philosophical Society Transactions, 1851. IX: p. 8-106.

102. Mazur, P. and D. Bedeaux, A generalization of Faxen's theorem to nonsteady motion of a sphere through an incompressible fluid in arbitrary flow. Physica, 1974. 76: p. 235-246.

103. Landau, L.D. and E.M. Lifshitz, Fluid mechanics. Course of theoretical physics. Vol. 6. 1987, NEW YORK: Pergamon Press. 539.

104. Bedeaux, D. and P. Mazur, Brownian motion and fluctuating hydrodynamics. Physica, 1974. 76(2): p. 247258. 
105. Jeffrey, D.J., Low-Reynolds-number flow between converging spheres. Mathematika, 1982. 29(57): p. 5866.

106. Jeffrey, D.J. and Y. Onishi, Calculation of the resistance and mobility functions for two unequal rigid spheres in low-Reynolds-number flow. Journal of Fluid Mechanics, 1984. 139: p. 261-290.

107. Liverpool, T.B. and F.C. MacKintosh, Inertial effects in the response of viscous and viscoelastic fluids. Physical Review Letters, 2005. 95(20).

108. Meiners, J.C. and S.R. Quake, Direct measurement of hydrodynamic cross correlations between two particles in an external potential. Physical Review Letters, 1999. 82(10): p. 2211-2214.

109. Atakhorrami, M., et al., Short-time inertial response of viscoelastic fluids: Observation of vortex propagation. Physical Review Letters, 2005. 95(20).

110. Atakhorrami, M., et al., Short-time inertial response of viscoelastic fluids measured with Brownian motion and with active probes. Physical Review E, 2008. 77(6).

111. Deen, W.M., Analysis of transport phenomena. Topics in Chemical Engineering, ed. K.E. Gubbins. 1998, NEW YORK: Oxford University Press.

112. Henderson, S., S. Mitchell, and P. Bartlett, Direct measurements of colloidal friction coefficients. Physical Review E, 2001. 64(6).

113. Bartlett, P., S.I. Henderson, and S.J. Mitchell, Measurement of the hydrodynamic forces between two polymer-coated spheres. Philosophical Transactions of the Royal Society A, 2001. 359(1782): p. 883-893.

114. Crocker, J.C., Measurement of the hydrodynamic corrections to the Brownian motion of two colloidal spheres. Journal of Chemical Physics, 1997. 106(7): p. 2837-2840.

115. Reichert, M. and H. Stark, Hydrodynamic coupling of two rotating spheres trapped in harmonic potentials. Physical Review E, 2004. 69(3).

116. Dufresne, E.R., et al., Hydrodynamic coupling of two Brownian spheres to a planar surface. Physical Review Letters, 2000. 85(15): p. 3317-3320.

117. Hunter, R.J., Recent developments in the electroacoustic characterisation of colloidal suspensions and emulsions. Colloids and Surfaces A, 1998. 141(1): p. 37-66.

118. Lukic, B., et al., Direct observation of nondiffusive motion of a Brownian particle. Physical Review Letters, 2005. 95(16).

119. Paul, M.R., M.T. Clark, and M.C. Cross, Coupled motion of microscale and nanoscale elastic objects in a viscous fluid. Physical Review E, 2013. 88(4).

120. Ducker, W.A., T.J. Senden, and R.M. Pashley, Measurement of forces in liquids using a force microscope. Langmuir, 1992. 8(7): p. 1831-1836.

121. Hutter, J.L. and J. Bechhoefer, Calibration of atomic-force microscope tips. Review of Scientific Instruments, 1993. 64(7): p. 1868-1873.

122. Honig, C.D.F. and W.A. Ducker, Squeeze Film Lubrication in Silicone Oil: Experimental Test of the NoSlip Boundary Condition at Solid-Liquid Interfaces. Journal of Physical Chemistry C, 2008. 112(44): p. 17324-17330.

123. Honig, C.D.F., et al., Lubrication forces in air and accommodation coefficient measured by a thermal damping method using an atomic force microscope. Physical Review E, 2010. 81(5).

124. Liang, X.G., H. Kuhn, and M.D. Frank-Kamenetskii, Monitoring single-stranded DNA secondary structure formation by determining the topological state of DNA catenanes. Biophysical Journal, 2006. 90(8): p. 2877-2889.

125. Ashton, N.W., et al., Human single-stranded DNA binding proteins are essential for maintaining genomic stability. Bmc Molecular Biology, 2013. 14(9): p. 1-20.

126. Marceau, A.H., Functions of single-strand DNA-binding proteins in DNA replication, recombination, and repair, in Single-Stranded DNA Binding Proteins, J.L. Keck, Editor. 2012, Springer, Humana Press: NEW YORK. p. 1-21.

127. Forties, R.A. and R. Bundschuh, Modeling the interplay of single-stranded binding proteins and nucleic acid secondary structure. Bioinformatics, 2010. 26(1): p. 61-67.

128. Merrin, J., P. Kumar, and A. Libchaber, Effects of pressure and temperature on the binding of RecA protein to single-stranded DNA. Proceedings of the National Academy of Sciences of the United States of America, 2011. 108(50): p. 19913-19918.

129. Witte, G., C. Urbanke, and U. Curth, Single-stranded DNA-binding protein of Deinococcus radiodurans: a biophysical characterization. Nucleic Acids Research, 2005. 33(5): p. 1662-1670. 
130. Robbins, J.B., et al., The euryarchaeota, nature's medium for engineering of single-stranded DNA-binding proteins. Journal of Biological Chemistry, 2005. 280(15): p. 15325-15339.

131. Chan, K., et al., Base Damage within Single-Strand DNA Underlies In Vivo Hypermutability Induced by a Ubiquitous Environmental Agent. PLoS Genetics, 2012. 8(12).

132. Ha, T., A.G. Kozlov, and T.M. Lohman, Single-Molecule Views of Protein Movement on Single-Stranded DNA, in Annual Review of Biophysics. 2012, Annual Reviews: PALO ALTO, CALIFORNIA. p. 295-319.

133. Chen, H., et al., Ionic strength-dependent persistence lengths of single-stranded RNA and DNA. Proceedings of the National Academy of Sciences, 2012. 109(3): p. 799-804.

134. Meisburger, S.P., et al., Polyelectrolyte Properties of Single Stranded DNA Measured Using SAXS and Single-Molecule FRET: Beyond the Wormlike Chain Model. Biopolymers, 2013. 99(12): p. 1032-1045.

135. Mills, J.B., E. Vacano, and P.J. Hagerman, Flexibility of single-stranded DNA: Use of gapped duplex helices to determine the persistence lengths of poly(dT) and poly(dA). Journal of Molecular Biology, 1999. 285(1): p. 245-257.

136. Sim, A.Y.L., et al., Salt dependence of the radius of gyration and flexibility of single-stranded DNA in solution probed by small-angle x-ray scattering. Physical Review E, 2012. 86(2).

137. Rivetti, C., C. Walker, and C. Bustamante, Polymer chain statistics and conformational analysis of DNA molecules with bends or sections of different flexibility. Journal of Molecular Biology, 1998. 280(1): p. 4159.

138. Rechendorff, K., et al., Persistence length and scaling properties of single-stranded DNA adsorbed on modified graphite. Journal of Chemical Physics, 2009. 131(9).

139. Zhang, Y., H.J. Zhou, and Z.C. Ou-Yang, Stretching single-stranded DNA: Interplay of electrostatic, basepairing, and base-pair stacking interactions. Biophysical Journal, 2001. 81(2): p. 1133-1143.

140. Wang, F.H., Y.Y. Wu, and Z.J. Tan, Salt contribution to the flexibility of single-stranded nucleic acid offinite length. Biopolymers, 2013. 99(6): p. 370-381.

141. Humphris, A.D.L., J. Tamayo, and M.J. Miles, Active quality factor control in liquids for force spectroscopy. Langmuir, 2000. 16(21): p. 7891-7894.

142. Humphris, A.D.L., et al., Transverse dynamic force spectroscopy: A novel approach to determining the complex stiffness of a single molecule. Langmuir, 2002. 18(5): p. 1729-1733.

143. Uzawa, T., et al., The Length and Viscosity Dependence of End-to-End Collision Rates in Single-Stranded DNA. Biophysical Journal, 2009. 97(1): p. 205-210.

144. Radiom, M., et al., A correlation force spectrometer for single molecule measurements under tensile load. Journal of Applied Physics, 2013. 113(1).

145. Smith, S.B., Y.J. Cui, and C. Bustamante, Overstretching B-DNA: The elastic response of individual double-stranded and single-stranded DNA molecules. Science, 1996. 271(5250): p. 795-799.

146. Stirnemann, G., et al., Elasticity, structure, and relaxation of extended proteins under force. Proceedings of the National Academy of Sciences, 2013. 110(10): p. 3847-3852.

147. Yang, W.Y. and M. Gruebele, Detection-dependent kinetics as a probe of folding landscape microstructure. Journal of the American Chemical Society, 2004. 126(25): p. 7758-7759.

148. Murayama, Y., Y. Sakamaki, and M. Sano, Elastic response of single DNA molecules exhibits a reentrant collapsing transition. Physical Review Letters, 2003. 90(1).

149. Murayama, Y., H. Wada, and M. Sano, Dynamic force spectroscopy of a single condensed DNA. Epl, 2007. 79(5).

150. Lapidus, L.J., W.A. Eaton, and J. Hofrichter, Measuring the rate of intramolecular contact formation in polypeptides. Proceedings of the National Academy of Sciences, 2000. 97(13): p. 7220-7225.

151. Alexander-Katz, A., H. Wada, and R.R. Netz, Internal Friction and Nonequilibrium Unfolding of Polymeric Globules. Physical Review Letters, 2009. 103(2).

152. Kapon, R., R. Nevo, and Z. Reich, Protein energy landscape roughness. Biochemical Society Transactions, 2008. 36: p. 1404-1408.

153. Chiti, F. and C.M. Dobson, Protein misfolding, functional amyloid, and human disease. Annual Review of Biochemistry, 2006. 75: p. 333-366.

154. McCauley, M.J. and M.C. Williams, Review: Optical tweezers experiments resolve distinct modes of DNA-protein binding. Biopolymers, 2009. 91(4): p. 265-282.

155. Gross, P., et al., Quantifying how DNA stretches, melts and changes twist under tension. Nature Physics, 2011. 7(9): p. 731-736. 
156. James, G.K. and J.Y. Walz, Hydrodynamic Force on a Microparticle Approaching a Wall in a Nanoparticle Dispersion: Observation of a Separation-Dependent Effective Viscosity. Langmuir, 2012. 28(1): p. 92-103. 